\title{
After The Protests: A Campus Racial Climate Case Study of the Perception and Curricular Responses For Institutional Reforms, Following the Black Students' Demands For Interventions at the

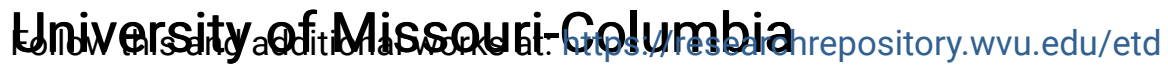

Brucert. Maftechellerican Politics Commons, Cognition and Perception Commons, Community College

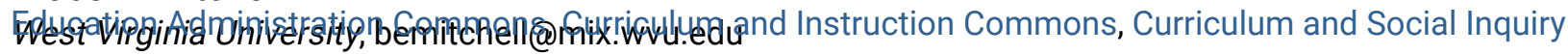
Commons, Demography, Population, and Ecology Commons, Disability and Equity in Education Commons, Educational Assessment, Evaluation, and Research Commons, Educational Leadership Commons, Educational Psychology Commons, Elementary and Middle and Secondary Education Administration Commons, Higher Education Commons, Higher Education Administration Commons, Human Ecology Commons, Inequality and Stratification Commons, Politics and Social Change Commons, Quantitative, Qualitative, Comparative, and Historical Methodologies Commons, Race and Ethnicity Commons, Secondary Education Commons, Social and Philosophical Foundations of Education Commons, Social Justice Commons, Social Psychology and Interaction Commons, and the Teacher Education and Professional Development Commons

\section{Recommended Citation}

Mitchell, Bruce E. II, "After The Protests: A Campus Racial Climate Case Study of the Perception and Curricular Responses For Institutional Reforms, Following the Black Students' Demands For Interventions at the University of Missouri-Columbia" (2021). Graduate Theses, Dissertations, and Problem Reports. 8073.

https://researchrepository.wvu.edu/etd/8073

This Dissertation is protected by copyright and/or related rights. It has been brought to you by the The Research Repository @ WVU with permission from the rights-holder(s). You are free to use this Dissertation in any way that is permitted by the copyright and related rights legislation that applies to your use. For other uses you must obtain permission from the rights-holder(s) directly, unless additional rights are indicated by a Creative Commons license in the record and/ or on the work itself. This Dissertation has been accepted for inclusion in WVU Graduate Theses, Dissertations, and Problem Reports collection by an authorized administrator of The Research Repository @ WVU. For more information, please contact researchrepository@mail.wvu.edu. 


\section{AFTER THE PROTESTS: A CAMPUS RACIAL CLIMATE CASE STUDY OF THE PERCEPTIONS AND CURRICULAR RESPONSES FOR INSTITUTIONAL REFORMS, FOLLOWING THE BLACK STUDENTS' DEMANDS FOR INTERVENTIONS AT THE UNIVERSITY OF MISSOURI-COLUMBIA}

Bruce E. Mitchell II

Follow this and additional works at: https://researchrepository.wvu.edu/etd

Part of the American Politics Commons, Cognition and Perception Commons, Community College Education Administration Commons, Curriculum and Instruction Commons, Curriculum and Social Inquiry Commons, Demography, Population, and Ecology Commons, Disability and Equity in Education Commons, Educational Assessment, Evaluation, and Research Commons, Educational Leadership Commons, Educational Psychology Commons, Elementary and Middle and Secondary Education Administration Commons, Higher Education Commons, Higher Education Administration Commons, Human Ecology Commons, Inequality and Stratification Commons, Politics and Social Change Commons, Quantitative, Qualitative, Comparative, and Historical Methodologies Commons, Race and Ethnicity Commons, Secondary Education Commons, Social and Philosophical Foundations of Education Commons, Social Justice Commons, Social Psychology and Interaction Commons, and the Teacher Education and Professional Development Commons 
Bruce E. Mitchell II,

\author{
A Dissertation \\ Submitted to the College of Education and Human Services \\ At West Virginia University \\ In partial fulfillment of the requirements of the degree of \\ Doctor of Philosophy in \\ Educational Leadership and Policy Studies \\ Nathan Sorber, Ph.D. Chair \\ Erin McHenry-Sorber, Ph.D. \\ Abhik Roy, Ph.D. \\ Sam Stack, Ph.D. \\ Department of Curriculum and Instruction
}

Morgantown, West Virginia

2021

Keywords: Campus-racial-climate, curricular-reform/interventions, Intergroup-Dialogue, Mizzou, 2015protests, Citizenship@Mizzou, Race, American Story, student-demands

Copyright 2021 Bruce E. Mitchell II 


\section{Abstract \\ AFTER THE PROTESTS: A CAMPUS RACIAL CLIMATE CASE STUDY OF THE PERCEPTIONS AND CURRICULAR RESPONSES FOR INSTITUTIONAL REFORMS, FOLLOWING THE BLACK STUDENTS' DEMANDS FOR INTERVENTIONS AT THE UNIVERSITY OF MISSOURI-COLUMBIA}

Author: Bruce E. Mitchell II, Ph.D.

This qualitative method single case study explores the phenomenon of a racially tense campus climate at the University of Missouri Columbia, a Predominantly White Midwestern Institution. At the forefront of the media regarding student and athlete protests, leading to the resignation of senior level administrators, African American students put forth eight demands to their administrators. Included, was the creation and implementation of a required racial awareness and inclusion curriculum. The study explores the perceptions of the institutional response to an exceptional campus racial climate issue and the process of formulating and participating in a diversity training course and a semester long course centered around race. Also, to understand how participants perceived this curricular intervention to have addressed their discontinuities in race and racism and contributed to organizational change, and institutional reform. This study addresses a gap in our understanding of the aftermath of such events, the institutional process of responding to them, addresses an understudied area of divergent participant perceptions, by exploring how student's demands were implemented as institutional interventions, and by understanding their perceptions of building a required racial awareness and inclusion curriculum. Four-emergent themes: Process of Curricular Reform Implementation, Towards Combating Discontinuities of Intergroup Relations in American Society at Mizzou through Education, Required or Optional Racial Awareness Curriculum to Elicit Institutional Reforms, and Perceptions about the Institutional Response/Curricular Reform Efforts to Change Mizzou's Campus Racial Climate. Hence, I developed a framework for considerations: Towards Implementing a Race and Identityfocused Inclusive Curriculum, for practitioners/educators desirous of implementing said curriculum. 


\title{
Acknowledgments
}

\author{
My Gratitude Belongs to You
}

To imagine gathering my thoughts to assign acknowledgements to the things and individuals who have aided me through a six-year storm, is undoubtedly a huge feat to fathom. I do so at the risk of relying upon a mind incapable of remembering all the little but so vital things that gave me solace to persevere. A mind, along the way, that has been tattered, rocked, shaken, bewildered and enlightened, is likely to stumble a bit at capturing the full scope of its experiences. But know that my heart earnestly seeks to show gratitude, gratitude to the people, places and things, no matter how big or small. My heart is forever grateful for the things that many of my loved ones have done or said to me to weather this academic storm. I am grateful for the "People in My Life" (A.T. Parker), who, at times didn't know it, took turns talking me through the turbulence, even when some could not hear the pressure of the wind in my background or see the stress of the loneliness and despair upon my face. It was some of you who made me smile, who made me laugh, who gave me an outlet, and who let me be vulnerable about the pressures of my journey. My gratitude belongs to you. Little do you know, it gave me resolve, fortitude, and a renewed sense of motivation to come face-to-face, once again, with the title of my high school commencement address, "The Power of Perseverance," as I was forced to own the essence of its true meaning to possess the power necessary to persevere my metaphorical storm. Likewise, I found myself lacking the confidence and direction I needed to get to the other side of the lightening and past the dark clouds where I could see the sun again, or in this case, a successfully defended dissertation. It was hard to imagine a smooth landing because I was so far up in the sky, all I could see were clouds of obstacles and all I could feel was turbulence. But the song writers Missy "Misdemeanor" Elliott and Ginuwine penned a song that says, "I'm the stewardess of the plane, feel the turbulence and maintain, please refrain, stay in your seats, until we reached the gate." Now I can say, I have felt the turbulence, I maintained, I refrained from ending my journey, I stayed the course and I've reached the gate! So, to "the people," thank you, thank you, thank you, for supporting me along the way. Your time spent talking, lifting me up, and pouring into me when I was weaker than I ever would have imagined, does not and will not go unnoticed; for my gratitude belongs to you. 
My Gratitude Belongs to...

\section{God}

If it had not been for the Lord, who was on my side, where would I be? Dear God, you have sustained me throughout this entire journey, even when I failed to cast all my cares upon you and seek you first for guidance and direction. You woke me up every day with the activity of my limbs, breath in my body and although fragile at times, a right mind. Even after I faulter and stray, you hear my cry and let me feel your presence consume my body to comfort me. And for this, I "give thanks with a grateful heart, to the Holy One." In this next phase, I need you to bless my soul and order my next steps, for "in time" God's time, will our change come.

\section{Mother}

Ma, Victoria Ballentine, I know not where to begin. Thank you for showing and expressing

"A Mother's Love." A love that does not waiver, a love that does not neglect, a love that is unconditional, undying, countless, and has no end in sight. I thank you for everything you have done, all the sacrifices you've made to make me happy and feel supported, all of the long talks on the phone, the infamous "kitchen talks" on holidays and when I return to Cleveland. Thank you for believing in me and fighting for me to the end!' A mother's love is unmatched, and I thank God

for keeping you here with me, I cannot image my life without your love, laughs and presence.

\section{Father}

Daddy, Bruce E. Mitchell Sr., we have felt the turbulence, but have yet maintained. Thank you for the check-ins and talks through the pandemic; they strengthened our bond and, at times, gave me an outlet from the journey. Thank you for setting the bar for what financial security could

look like in Black families. It is because of you and your industrious nature and hard work in multiple professions that I strive to one day possess your enterprising nature to solidify my ability

to be independent and resourceful in all my endeavors. Without your current sacrifices and ongoing financial support throughout this process, I would not be capable of getting where I am now. I know I asked a lot but know that the harvest will be plentiful.

\section{Family}

Thank you to the James and Mitchell immediate and extended family members, who hyped me up throughout this entire journey. A special thank you to a few of my aunts and cousins who have praised me like I was the baddest thing since sliced bread! When I didn't have confidence in myself, that helped. A special thank you to my aunts Joline, Gwen and my adopted aunt Traci, who have provided financial support along the way; great was your love and kindness toward me. Auntie Gwen, you have supported my academic career path since Kent State, sending me love tokens on a regular basis to show your value in my educational journey. Aunt Traci, you

have managed to stun me every time you send your blessings and I'm so grateful for you contributing in such a big way to my needs. Honorable mention: My "Janet Jackson" Sherese

"Althea” Ballentine (Sister), Tristen Burrell Ballentine (Niece), Nathan Smart (Nephew). In loving memory: Grandparents: Coleman \& Victoria James, Melvin \& Lucille Mitchell, Aunt Hattie Davis, Uncles: MacArthur \& Coleman James, Brother: Eric Smart.

A huge thank you to my Brother Mike Daniels. When I started this journey over six years ago, we sat in a Cracker Barrel restaurant in Morgantown, West Virginia, for hours upon hours trying to narrow down my research focus. After each conversation, I came closer and closer to shaping my interests. Having been your mentor, it was initially hard to be open about my lack of confidence and areas of weakness, but you made it clear that none of that mattered and I could count on you for support at some of my lowest moments no matter how busy you were. You have no idea how much your words of advice, encouragement, and our joint prayers changed my life, my thinking, or my emotional state EVERY time we talked. God is using you in mighty ways Spec. 
Middle and High School Best Friends

Geoffrey: We may not talk often, but $6^{\text {th }}$ grade is a long time to rock with someone. You have always let me be me and had my back through the years. I know that if I needed you, you would be there!

Shawn: From the Black pool of genius, my laughing partner, you always amaze me with just the right things to say at my lowest points, your logic is unwavering, and your wisdom is like magic because of its power. From a different career path, you cared enough to be interested in my research, make inquires, and be invested in my doctoral journey. You motivated me, pushed me, and teased me into finishing this journey Bro., because you know how I think, and you knew

exactly how to address my points of dismay. You are my brother and I appreciate you!

Chris: Words lack the rigor needed to describe our bond and the emotional support you have given me through these years aside from school. Talking to you nearly every day made my days better, more fulfilled, and allowed me to be unapologetically me! It was YOU who got me through my lowest point and remained a very present help in a time of need. Our talks and our bond are like no other, you are my brother and I thank God for having you in my life!

Melanie: Your excitement and enthusiasm about my doctoral journey was through the roof! To know how much you believed in me and respected my pursuit was an affirmation to my desire to complete this journey. Our nudges and our laughs will always be a staple in our bond. It was you who told me that I was "A cultivator of Black Excellence!"

Diarra: Over this past year, you showed me so much love that it is, yet, left unmatched! Thank you for all your shout outs to show love and hype me up during this process, the laughs, and encouragement. I am so glad to have you in my life, pushing me through without judgement and I thank God for you, your hospitality, your loyalty and giving nature!

Honorable mention: Tenell Smith, always in my heart Bro.

\section{Mentees and Great Friends}

Korlin: My G-son, one of my first KSU Academic STARS mentees since 2003, and best friends. Over the past year, our bond has grown stronger. Your indirect support through our daily phone conversations has kept me going and were a highlight of my day after writing. When I second guessed myself on being qualified for interviews, you poured into my puddle full of confidence until it became a pond. When the cares of life and the pressure of this journey weighed me down, your listening ears lifted the strain.

Aaron Radcliffe: $M y$ G-son and BRIDGE 08 mentee, your visits to $W V$, the get-away trips and "Tour de Denver" were much needed outlets for me and will remain joyous memories in the midst of my journey. You are my son PERIOD. Your appreciation for what I have poured into you over the years, was reassuring to me and a very present help in the time of a storm.

Donnique: My WVU Academic STARS 2012 mentee, you, Ardell, Kendra, and Ikegai have been a solid care and support system for me when I needed good laughs, good food and good gossip. In the past, you all have stepped in to help me move and set up my new home. Donnique, your respect for me, your check-ins on the doctoral journey/my emotional state, and unwavering loyalty is supreme. Thank you for your kindness and giving spirit! 
Kimelle: My mentee, little sis, and Delta SPECial. Since your start at WVU, you have allowed and trusted me to mentor you. Thank you for having always showed up at my events when I needed you the most and helping me carry the social justice or gospel choir mission. Thank

you for respecting me enough to allow me to fuss at you when you needed direction and taking heed to my advice. "My little Black Studies Professor to be"

Chris Allen, Mike Harris, Casey Wokocha, and Isaiah Keys: The men of Kappa Alpha Psi Fraternity Inc., each of you have played a huge role in my success as a university administrator. In your own ways, you all have poured into me, motivated me, showed gratitude, allowed me to mentor you and even fuss at you when I needed to teach tough lessons for your own development. Chris and Mike, you both have literally brought me to tears with your gratitude towards me; it picked me up and kept me going!

Honorable mention to many of my mentees and great friends who have poured into me, showed expressions of gratitude when I needed it the most, include but are not limited to: Michelle, Stephanie, Kevin Louis, Ricardo, George, Avery, Rhedus, Jeremy, Zach (G-son), Ashley W., Ben, MY LIVV! (Olivia), Dierre, Maurice and you!

\section{Alpha Phi Alpha Fraternity Inc. Special Shout out to my line brothers and sands: IMPOSSIBLE / Spring 2004 / Epsilon Delta Chapter: LeAnder, Kareem (Kolade), and Chris.}

Brother James C. Johnson: My Alpha SPECial, Little Bro, and protégé. Since your time in high school, you have made it a point to check-in on me and keep me abreast of your collegiate journey. Thank you for all the words of encouragement you have given me over time, the public gratitude you showed me in 2018 when you knew I needed it most. Thank you for making me get out of bed to go write by the looks you gave me on FaceTimed when the hour grew late, and for taking initiative to ask me what I needed your help with for LDI when things got hectic. I thank God for having you in my life and for the personal and professional talks that has made our bond close.

Brother Khufu Edwards: My Alpha SPECial, mentee, and one of my best friends, what a journey it has been. I never knew that after all the pouring into you I did, I would need to-for a season-rely so much on you along this journey and during some of the toughest times of my life. You may not know it, but I think God was using you as you poured into me and extended true

brotherly love. Thank you for always having my back, accepting me for who I am without judgment and allowing me to be authentically me! I am so grateful to have you as a brother to talk to, vent to, share our personal stories, and master plans to transform the world one day. Know that the words you spoke did not fall on deaf ears, as they consumed my mind, infiltrated my understanding of alternative perspectives, and helped lift me from despair. I'll never forget your words in my despair, "we'll get through this together." When I stressed even the little things, it was you who told me that some things may be "a mood-changer but should not be a day-ender."

Brother William "Boojoe" Smith: My mentee and Alpha SPECial, your respect and loyalty to me is supreme! Over the past year, our bond has been strengthened through our almost-daily talks about life's twists and turns that have pressed us down a bit. You have watched me struggle through this process and allowed me to vent or just fellowship while you were at work and help with your meetings when I needed a break from writing. If it's one thing I know, "Boojoe" is a protective little bro and has my back! Thank you for flying in town to help me execute the Leadership Development Institute when I needed you; that meant the world to me! 
Brothers Quincy Smith and Jihad Dixon: My "Alpha boys," and my Academic STARS 13 mentee. Your journey in Alpha and the bonding trips with your dean lifted me from the doctoral path but for a moment, to see the light of Alpha again and the North Carolina Sun. Q, thank you for letting

me vent to you when people make me upset. Jihad, words may lack the power to express how grateful I am for your support and graphic or presentation assistance in almost every facet of my life - even my after life! When I have needed you, you have been a brother's brother and for that I am forever grateful.

Brother Arnold Roots: Given the demand of the doctoral experience, I faltered on my personal fitness goals and healthy eating habits. Thank you for driving from Fairmont to Morgantown every day to physically train me and pour into me when I got discouraged, wanted to give up on everything and when I needed someone to vent to and get feedback. Thank you for checking on me and being concerned about my health and well-being. I am impressed with your growth of wisdom and willingness to share and show brotherly compassion, you were literally saving my life.

Honorable mention to many of the Alphas I mentored, who made me or those of whom I made, they include, but are not limited to Brothers: Shannon Lanier, Shon, Ricardo, Dr. V. Skyes, Gene Shelton, Dr. Willis L. Lonzer, III (36 ${ }^{\text {th }}$ General President of Alpha Phi Alpha, who initiated me and had my back!), John Williams, Eufrancia, Dr. Jones, Ellis L., Ellis R., Jon Austin, Mike I., Andrew, Gibson, T. Vernon, Vernon, Corey, Julius, Tonek, Dr. Jones, Dr. Ottley, Dr. Le, Cannon, Kevin, Oterrious, Trevawn, Chris C., Dr. Quarles, Javier, Christian, Arnold, Ahkeem, Jacob, Keith, Derek, K. Ward, W. Harris, Kelvin, Zach, Adriel, Josh B., Isiah H., Luke, and Julian N.

\section{Kent State University}

Thank you to my entire support system at KSU! My undergraduate, graduate and first professional experiences there, afforded me a strong foundation in Higher Education and prepared me to fly.

First, a huge thank you to Shana M. Lee for accepting me into the Academic STARS Program in 2001 and for affording me the opportunities to mentor, teach, train staff, and keynote. You always listened to me as both a student and a professional and welcomed my ideas to advance the multicultural center. Thank you for trusting me to help you serve the students and for being such a supportive friend and colleague who has always had my back no matter what! "When the moon is not full, the STARS shine more brightly!"

A big thank you to Dr. Geraldine Hayes Nelson for giving me my first professional promotion and trusting me at such a young age to do this work! When I made mistakes, you didn't give up on me, you taught me lessons I didn't know I needed at the time, brushed me off and sent me back out and loved me no less, you had my back, and I am forever grateful! You are a TRUE advocate for students and the people! You gave us so many chances to be Black and Excellent!

Dr. Dana Lawless-Andric, you sat the committee that gave me my first professional job in HIED, you have been such an amazing supervisor and mentor through the years. You gave me a chance to shine, to restructure aspects of Upward Bound Classic, to discover and invest in my personal goals, and you gave me so many opportunities to operate in my calling at the university and with Ohio TRiO; pushing me to chair two state conferences! You prepared me for interviews

and still advise me on the professional and doctoral journey to this day and I will always be grateful for you being one of my biggest supporters. There are so many great people at Kent that I love, who have poured into me, I cannot share all the stories in this space but know that my love or appreciation for those who receive honorable mention is no less important that those above. 
Honorable mention: Dr. Fran Dorsey, Dr. Crosby, Traci Easley-Williams, Okantah, Nana Whitaker, Dean Moore, Gene Shelton, Dr. Sykes, Greg Jarvey, Eboni Pringle, Ms. Bonnie, Dr. T. Jefferson, Karen Clement, Trinidy Jeter, Yvonna WG., Dr. Drummer-Ferrell, Della M.M., Dr. Baker, Dr. King, Dr. Crawford, Dr. Brown, Dr. Michael, Dr. Tina L. Kandakai.

\section{West Virginia University}

Thank you to the team of individuals who first hired me at the $C B C \& R$, I will forever be grateful for the opportunities I had to serve the people, form strong bonds, and expand the scope of my experience professionally.

A big thank you to Vice President Emeritus, David M. Fryson, Esq. and President E.

Gordon Gee, for keeping me around to serve. VP Fryson, thank you for trusting me to do the work of DEI and social justice in a new division and for supporting and encouraging my doctoral

$$
\text { journey. }
$$

Dissertation Committee \& Faculty: A huge thank you to each of my committee members for helping me cross the finish line. I thoroughly enjoyed each of your classes through the years and will carry with me, the deep dialogues that guided our learning.

Dr. Sorber, thank you for serving as my chair and guiding me through this process, answering my many questions, putting my stresses to rest, and giving me positive feedback along the way. I enjoyed learning the history of Higher Education and strategic planning and budget; it was your class where I discovered my lineage as a descendant of John G. Mitchell, Founder of Wilberforce University.

Dr. Roy, words cannot express how grateful I am for the time you spent with me to help me narrow my research focus, craft my research design, and validate my feelings as a man of color navigating this journey; that alone, was vital to my success! Our meetings were therapeutic!

Dr. Malayna Bernstein, while you were not on my committee, you were there when I needed you most. You talked me through the mistreatment I experienced as a Black man following the 2016 election. Thank you for giving me the direction I needed when I was totally confused on the next steps to enter my data analysis and coding. I will always remember your passionate approach to teaching advanced qualitative methods.

Dr. Erin McHenry-Sorber, I thoroughly enjoyed all my courses with you, learned so much and am most grateful for the work I produced in your curriculum reform course, it gave me the room to develop a diversity education curriculum, of which I am so passionate about.

Dr. Sam Stack, I will always be grateful for the philosophical knowledge I gained from your Nature of Inquiry and Philosophy of Education courses. Thank you for always supporting me!

There is no way that I can end this section without recognizing a faculty member I did not meet until I reached out to her, in desperate need of guidance with coding and the use of the data analysis software.

Dr. Anupama Shekar is an evaluation consultant at Southern Methodist University in Dallas, Texas. She did not know me but agreed to meet with me virtually and give me the technical guidance needed to enter data analysis and for that, I will forever be grateful!

Honorable mention: Dr. Amena Anderson, Dr. Constinia Charbonette, Eric Jordan, Sharon Mallow, Dr. and Mrs. Epps, Rob Lyons, Jill, Sorors: Anitra, Rosie and Toni. 


\section{Table of Contents}

AFTER THE PROTESTS: A CAMPUS RACIAL CLIMATE CASE STUDY OF THE PERCEPTIONS AND CURRICULAR

RESPONSES FOR INSTITUTIONAL REFORMS, FOLLOWING THE BLACK STUDENTS' DEMANDS FOR

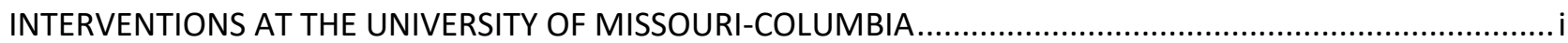

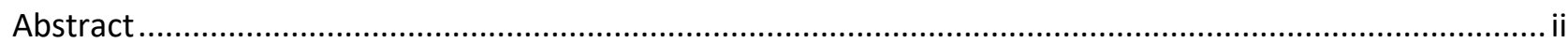

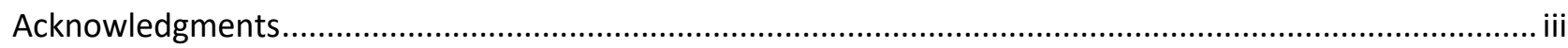

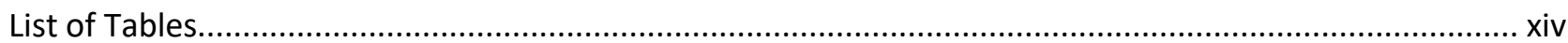

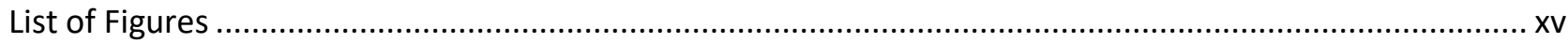

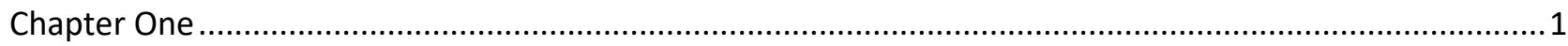

Introduction

The Case: A timeline of the preceding prompts to campus unrest .......................................................5

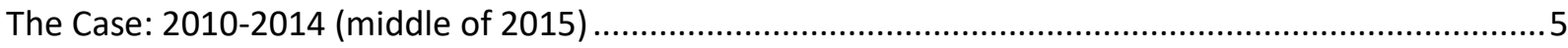

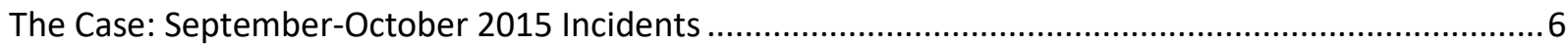

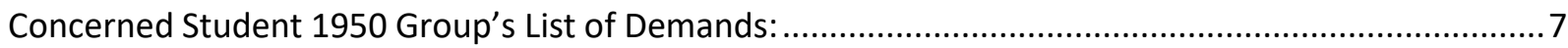

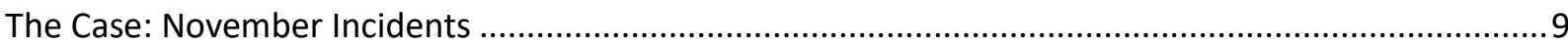

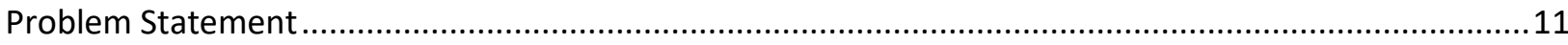

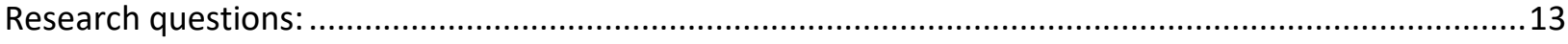

Introduction to racist and racially inequitable campus climates ..........................................................14

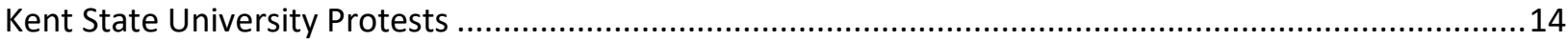

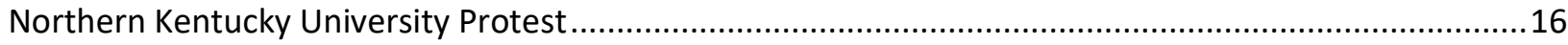

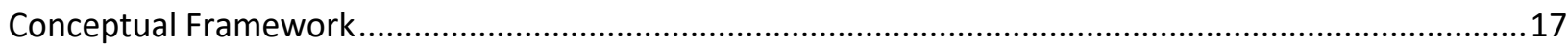

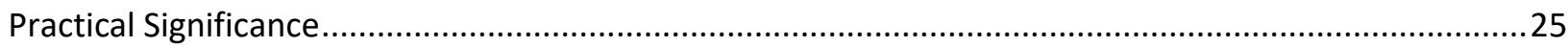

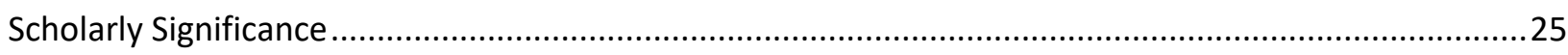

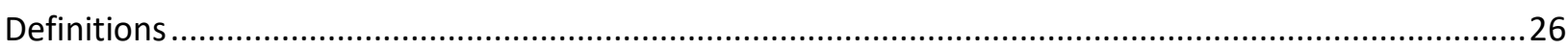

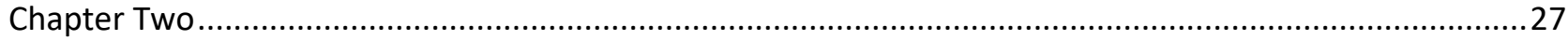

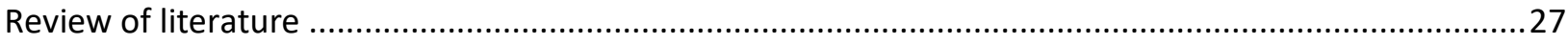

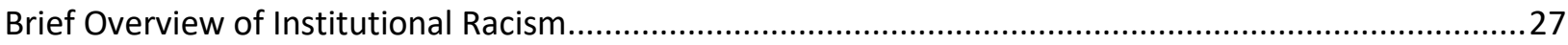




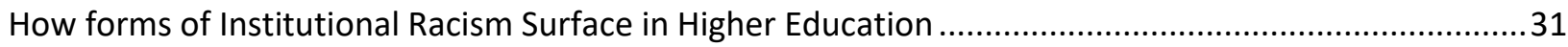

Toward a Better Campus Racial Climate from Institutional Racism in Higher Education...........................37

General Overview of Campus Racial Climate \& the Journey to Creating Inclusive Climates.....................40

A Closer Look at what Undergirds Black Student Demands and Activism .............................................50

Understanding Racial Identity Development for campus reform ..........................................................56

Curricular and Non-Curricular Reform Through Identity Development and Intergroup Dialogue................58

A Brief Understanding of The Individual Diversity Development Framework........................................59

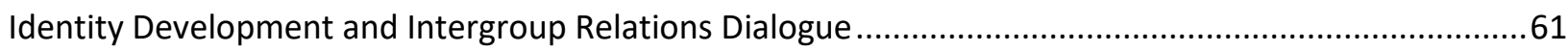

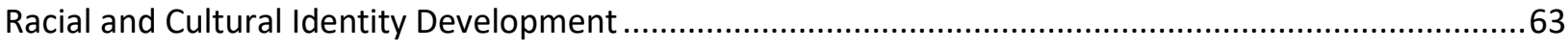

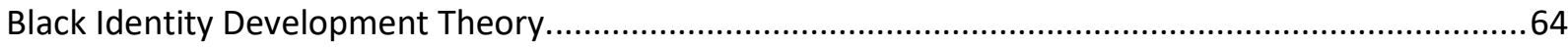

White Identity Development and White Racial Consciousness Models.............................................66

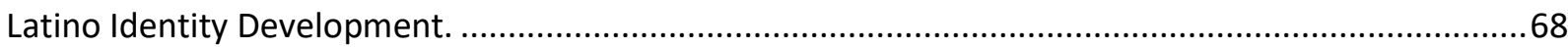

Curriculum and Student Organizations: Understanding more on Campus Reform ..................................69

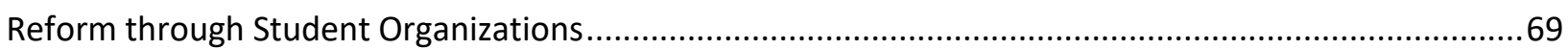

Service Learning, Intergroup Dialogue or Lecture-based Diversity Curricular Reform Approach .................70

Dedicated Diversity Course, Infusion Model or Egalitarian Approaches in the Curriculum .....................71

Understanding the Foundation of a Seminal Critical Pedagogy ...........................................................73

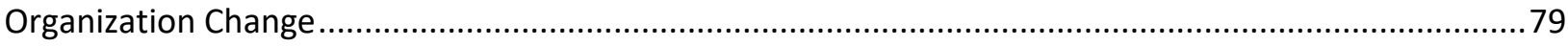

The Role of Chief Diversity Officers for Institutional Reform ..............................................................

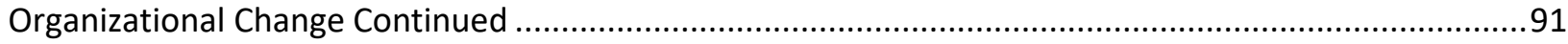

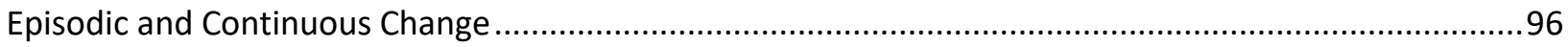

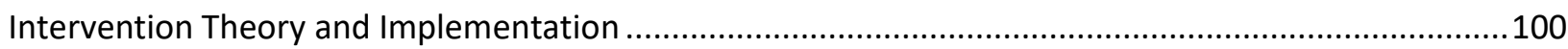

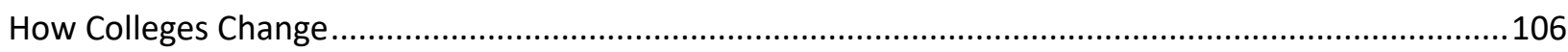

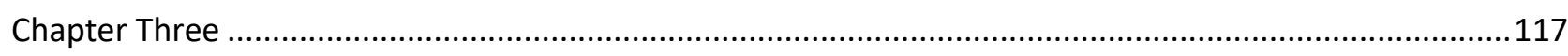

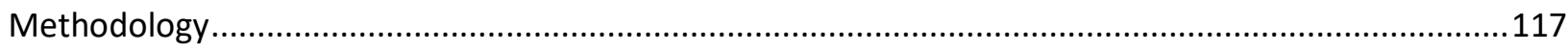

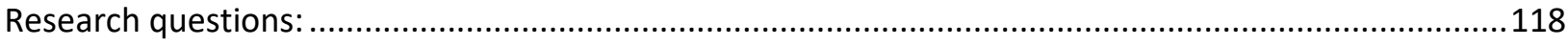

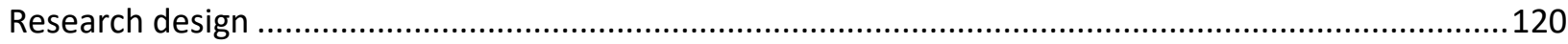

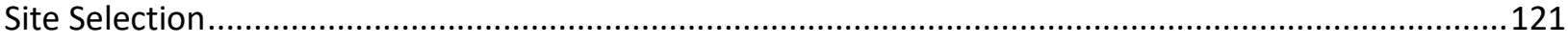

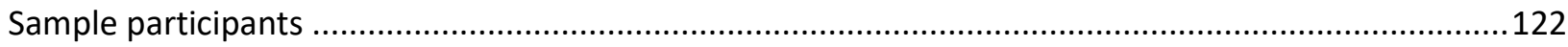

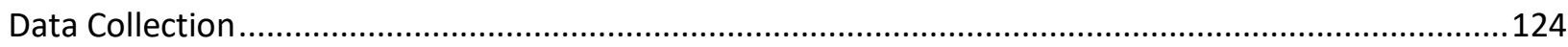

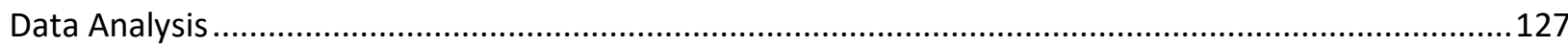


General Overview of Analysis 129

The Use of CRT and CRT/I for Analysis 130

Data Analysis using CRT and CRT/I in this Study .134

Validity of Research. 135

Researcher positionality 137

Chapter Four 139

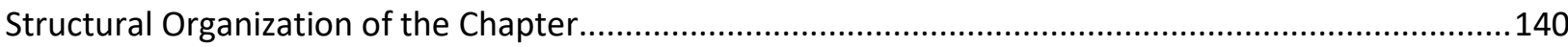

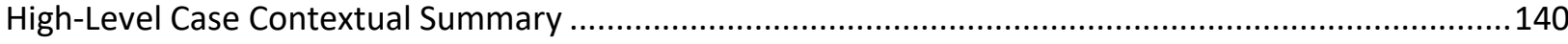

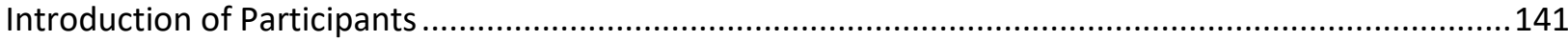

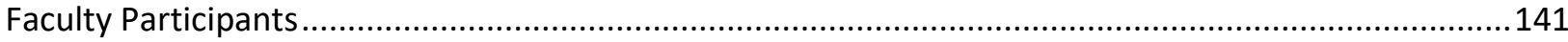

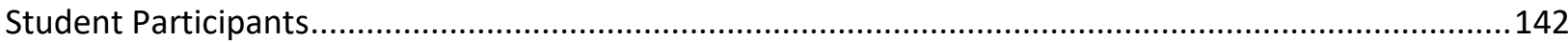

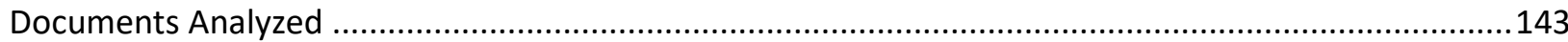

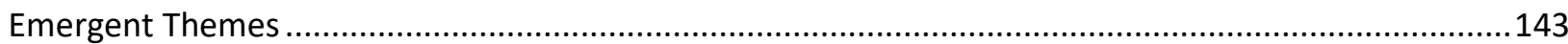

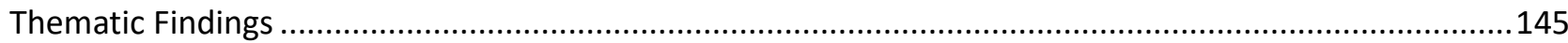

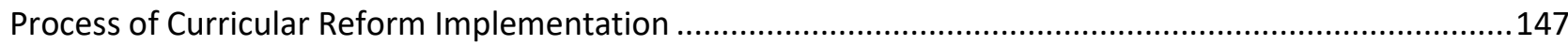

The Implementation Process of Citizenship@Mizzou ......................................................................160

The Implementation Process of Race and the American Story …...................................................173

Towards Combating Discontinuities of Intergroup Relations in American Society at Mizzou through

Education

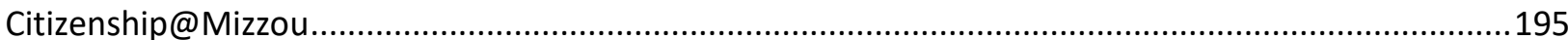

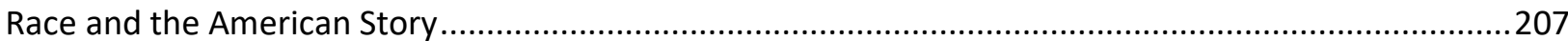

Required or Optional Racial Awareness Curriculum to Elicit Institutional Reform ...................................243

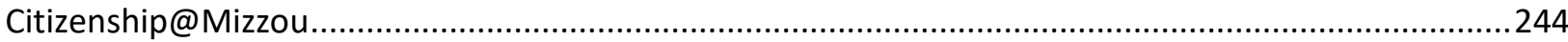

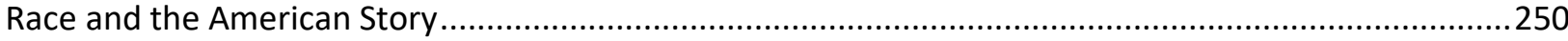

Perceptions about the institutional response / curricular reform efforts to change Mizzou's Campus Racial

Climate 


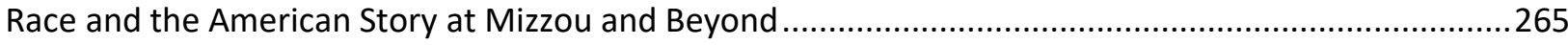

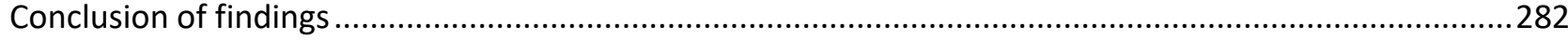

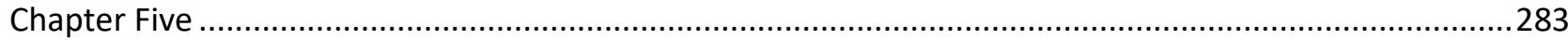

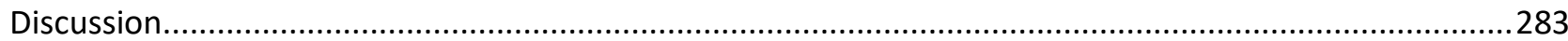

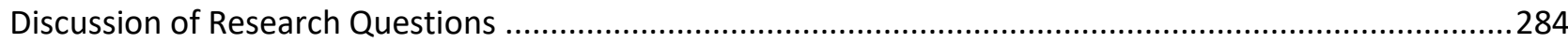

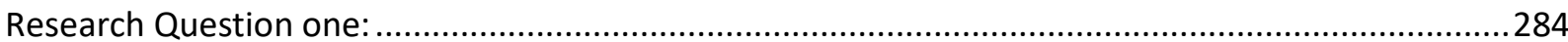

Research Question two:

Practical Implications for Higher Education and A General Framework of Considerations Model for

Implementing Race, Identity and Inclusion-focused Curricular Interventions ........................................297

Higher Education Senior Leaders and University Staff/Administrators...........................................298

Faculty, Deans, Department Chairs, Curriculum Committees, Human Resources and Hiring committees

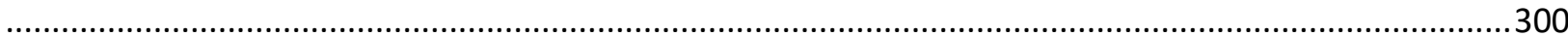

Towards Implementing a Race and Identity-Focused Inclusive Curriculum (TIRIFIC): .............................302

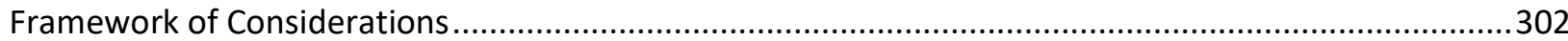

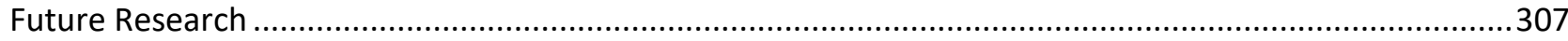

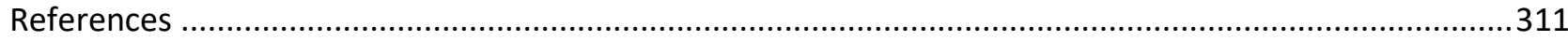

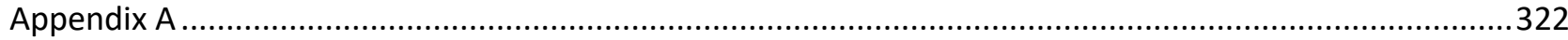

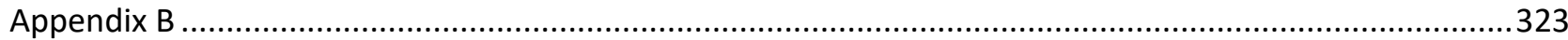

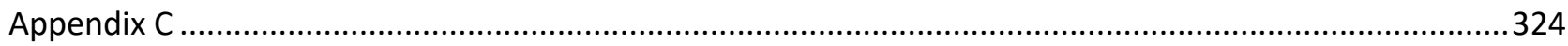

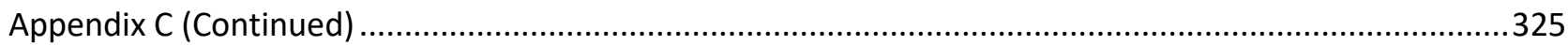

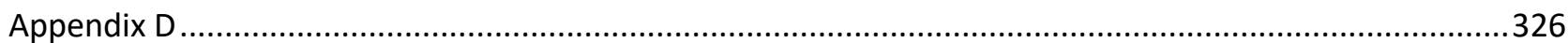

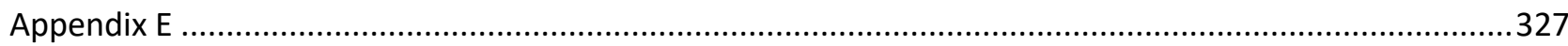

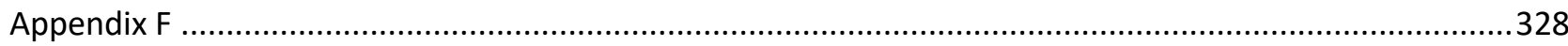




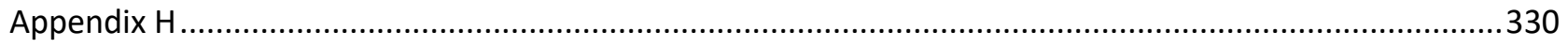

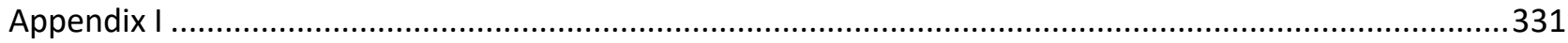

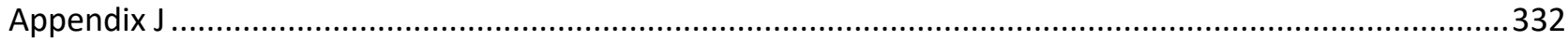

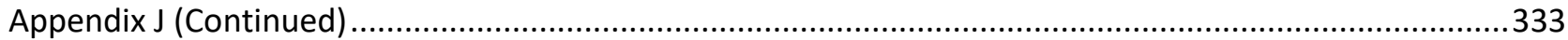




\section{List of Tables}

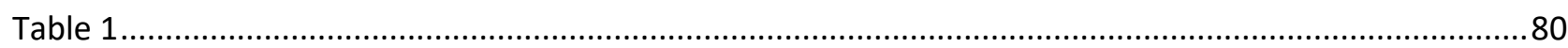

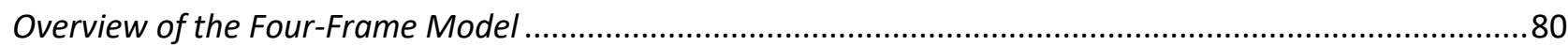

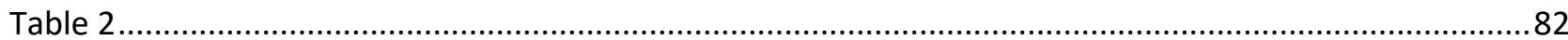

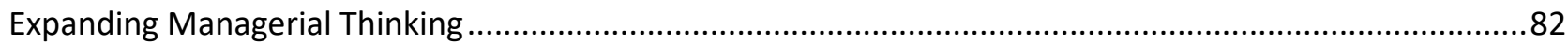

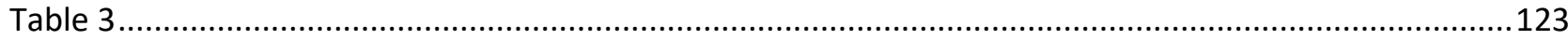

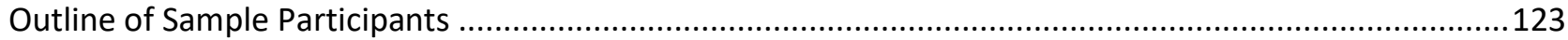

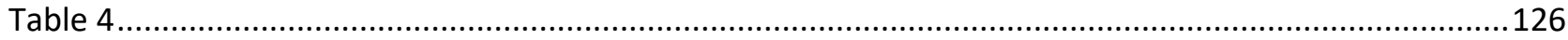

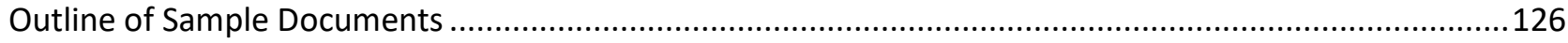

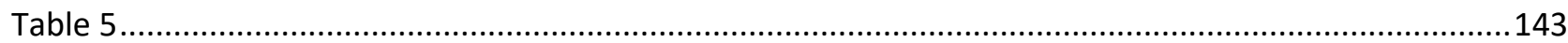

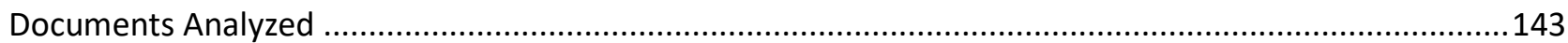

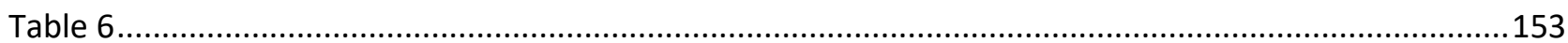

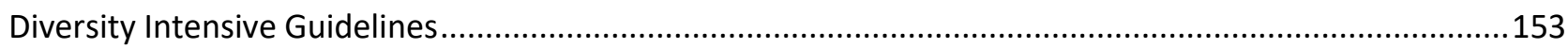




\section{List of Figures}

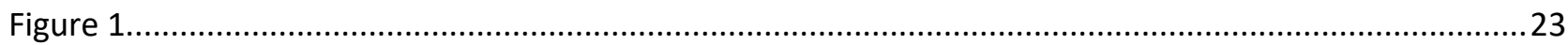

Bronfenbrenner's Bioecological Systems model with added Techo-subsystem .......................................22

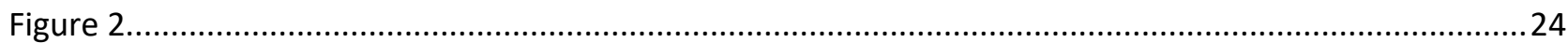

Networked Model of Ecological Systems, Focused on Person A.........................................................24

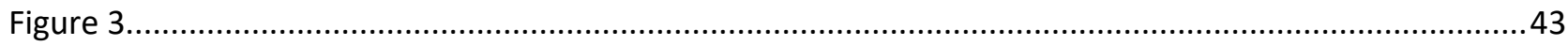

73 Colleges and universities across the United States ranked in order of frequency ..................................43

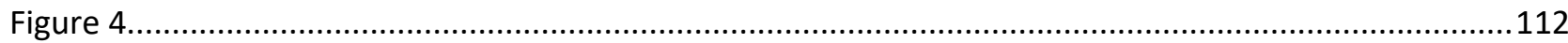

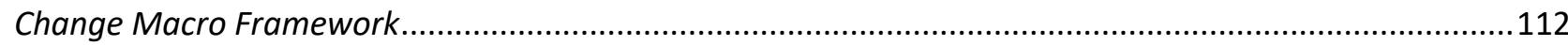

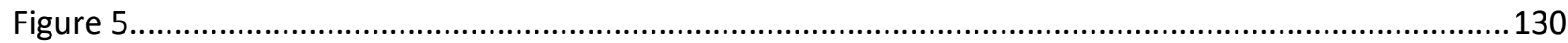

A streamlined codes-to-theory model for qualitative inquiry ..................................................................130

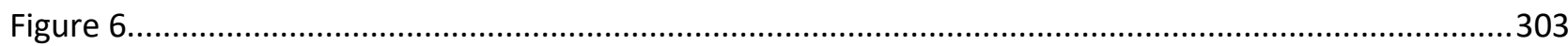

Towards Implementing a Race and Identity-Focused Inclusive Curriculum: Framework of Considerations

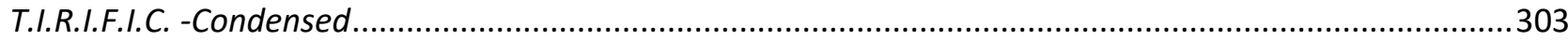

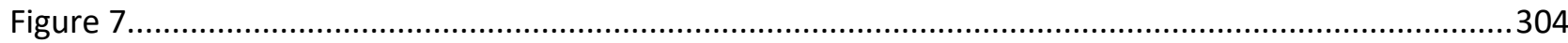

Towards Implementing a Race and Identity-Focused Inclusive Curriculum: Framework of Considerations

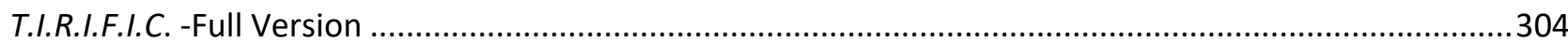




\section{Chapter One}

\section{Introduction}

The campus racial climate and ecological factors one experiences obtaining a post-secondary education contribute to a student's overall development and academic outcomes. Institutions of higher learning are where growing minds develop and behaviors are shaped by lived experiences. The exposure to new knowledge and varying intergroup relations contribute to this process of personal, professional, and academic development. Because of these interactions, people become more aware of and are influenced by the social and cultural values and beliefs that others ascribe to or define them. At the same time, individuals internalize these ideals which help them form more accurate perceptions of the others (Chavez, Guido-DiBrito, and Mallory,2003). From here, those who are exposed to intergroup relations can fashion their behavior in a way that does not cause them to ridicule or mistreat those around them, but rather understand and embrace other people and their identities.

Additionally, what students contend with or bring-literally or metaphorically-from home, influences the spaces they navigate on a university's campus. For example, if students are reared in a household that does not acknowledge privilege or, embraces or shows prejudice towards other cultures, they can bring this to any campus climate implicitly or explicitly. To validate this claim, Pinsker (2018), interviewed Margaret Hagerman, who authored White Kids: Growing Up with Privilege in a Racially Divided America, where she followed 36 children between the ages of 10 and 13 from upper-middle class White families. One participant of her study lamented "Racism is not a problem, it was a problem when all those slaves were around and that, like bus thing and the water fountain," while her mother nods in agreement (Pinsker, 2018). Additionally, Hagerman reported that while most White families stated they chose affluent neighborhoods and private schools because they wanted to be a good parent, she cautions us to consider the ways in which these choices disallow children the opportunity to experience and be immersed in 
culturally diverse spaces. Similarly, she stated that even those who sent their children to public schools, took them out immediately and placed them into costly private schools; such an option perpetuated the reality of their privilege to escape racism as White person, an option not afforded to people of color. To this end, Hagerman urges non-racist parents concerned with racial equity to consider how their privilege can be used to help change spaces that may not be "in the best interest of their child," but rather the right thing to do as a citizen. This represents a detachment from the biological responsibility as a parent to want what is best for their own child, to being concerned with the social construction of spaces for all children (Pinsker, 2018). On the other hand, Cabrera and Nora (1994) found that students of color were more attuned to forms of discrimination and prejudice than their White counterparts who were less likely to perceive gradations of it; differences within ethnic groups occurred depending upon their background or ethnic identity. Consequently, the variations of perceptions about discrimination and prejudice are important to note because perception is both a product of the environment and can determine future interactions (Astin, 1968; Tierney, 1987). Likewise, if students have had little to no exposure to racial otherness, it can be difficult to navigate campus spaces where they are now forced to reside, dine, or work collaboratively on a group project with others from different ethnicities or races (Chavez, Guido-DiBrito, and Mallory, 2003).

In drawing a connection to how these childhood experiences impact their current or future intergroup relations, Degner and Dalege (2013) reported a significant correlation between parental and child intergroup relations. In other words, children's and their parents' intergroup attitudes were found to be significantly related; however, the authors cautioned that some methodological issues and limited research on parental socialization of childhood prejudice impedes our ability to fully understand parents' impact on their child's intergroup attitudes and relations with other races. Nevertheless, what we do know from Rowe, Bennett and Atkinson's (1994) White Racial Consciousness Model (WRCM), is that White Racial Consciousness relates to "one's awareness of being White and what that implies in relation to those who 
do not share White group membership;" (Rowe et al., 1994 pp. 133-134) whereby they fall into or move between two types of categories: unachieved white racial consciousness and achieved white racial consciousness. According to Evans et al. (2010), people who "poses avoidant attitudes have not consciously thought about their race or racial experiences of other racial groups, often dismissing, ignoring, or avoiding race until forced to address their denial" (p. 262). Thus, it is likely that some White individuals, students, and faculty/administrators alike, might find themselves vacillating between the two types of categories. Torres, Howard-Hamilton \& Cooper (2003) asserted that a communication gap exists among faculty and administrators that lack knowledge about identity development. To further expound upon this idea, Hurtado, Milem, Clayton-Pedersen, and Allen's (1998) study revealed that when faculty and administrators make students feel valued and are devoted to their development, they are less likely to report racial/ethnic tension on campus. The findings suggest that student centered environments can limit racial tensions and competition among various social groups if campuses create the space for such engagement (Hurtado et al., 1998). Therefore, campus climate is impacted by a variety of unique factors; making it one that is either comfortable or an environment with racist tensions. Responsiveness to campus climate surveys and student demands may, in part, allow institutions to help address climate based upon the interventions derived from such demands (Hurtado et al., 1998). On the other hand, more vital than the faculty-student and staff-student engagement is the necessity to challenge community members to "deeply (re) examine their biases and assumptions about Blacks" (Harper, 2015, P. 667). To this end, Harper's (2015) study examined the campus climate and the racist stereotypes of Black males at PWIs; he argued that campus climates would be improved if White students and faculty had fewer stereotypes and made fewer racially offensive comments/micro-aggressions. Harper (2015) argues that opportunities for consciousness raising and corrective interventions are needed for Whites who, inadvertently or unwittingly impose racial harm on underrepresented students at PWIs. More recent literature addressed the notion of advancing a Critical cultural perspective to urge student affairs practitioners to become transformative 
educators. Rhoads and Black (2018) contend that the idea of a transformative education has been described as critical pedagogy, whereby focusing on how teachers might play a role in helping students be more reflective about how culture and social structure shapes their lives. Additionally, the goal is for students to develop a critical consciousness, engage in social and cultural transformation and work towards the creation of a more equitable and just society.

Across U.S. higher education, racist campus climates are proving to be problematic for students and faculty, staff, and administrators. This is no new phenomenon, as the historical legacies of racial inequities, oppression and marginality precede current trends in this regard. This dissertation expands knowledge to this field of research by examining the experiences in campus community in the aftermath of a racial climate event through the investigation of an exceptional case at the University of MissouriColumbia (Mizzou). Specifically, this dissertation investigated the process by which curricular interventions were pursued in response to the event and the perceptions and experiences of those involved in crafting and/or implementing them. The specific curricular interventions investigated in this dissertation were a required training course called Citizenship Mizzou and a one credit hour course called Race and the American Story. The course eventually was offered for three credit hours. Both courses were institutional responses to Black students' demand for a racial awareness and inclusion curriculum. The purpose of this study was to explore the perceptions of the institutional response to an exceptional campus racial climate issue at the University of Missouri and the process of formulating and participating in a diversity training course and a semester long course centered around race. Additionally, this dissertation sought to understand how participants perceived how this curricular intervention addressed discontinuities in race and racism on campus and contributed to organizational change, and institutional reform. 
The Case: A timeline of the preceding prompts to campus unrest

The Case: 2010-2014 (middle of 2015)

On February 26, 2010, two White students scattered cotton balls on the lawn of the Gaines/Oldham Black Cultural Center (GOBCC) at the University of Missouri Columbia for what was minimized by the participant as a "prank." This "prank," according to the deputy chancellor, Michael Middleton, caused other students to question their safety and belonging at Mizzou, for as one student lamented, “...somebody had disrespected my home" (Sunne, 2010). The students were sentenced by the Boone County Courthouse to two years of unsupervised probation and 80 hours of community service (Sunne, 2010). On August 9, 2014, about 120 miles from Mizzou in the town of Ferguson, Michael Brown, an unarmed Black teenager, was shot to death by a White police officer, Darren Wilson, igniting the formation of the Black Lives Matter movement (BLM) during a "Racism Lives Here" rally on September $24^{\text {th }}$ and keen discussions on racial campus unrest at campuses across the nation. Students from many universities across the nation stood in solidarity with Mizzou by organizing peaceful protests on their campuses and issuing demands for reform to their administrations (Pearson, 2015). The BLM movement drew national attention and grew into an international movement to demand justice, an end to an ongoing war on Black people, reparations, investment and divestment, economic justice, community control, and political power (Douglass and Shockley, 2017, p. 201). Douglass and Shockley (2017) situate the BLM agenda as consisting of "demands to a White power structure (e.g., government and business) for the purpose of making things fair and just for Black people," (p. 201) and in doings so, highlight ongoing injustices and recruit other reformers to the cause. Following the shooting death of Michael Brown and just before the start of the fall semester at Mizzou, three queer women (Black, Afro-Puerto Rican, and South Korean) led their peers in the first resistance act: a photoshoot called "Hands up, Don't Shoot," under the name \#MU4MikeBrown. These women continued with acts of resistance on campus and reached out to senior level administrators to request a private meeting with peer organizations to present 
a "Call to Action List" (McElderry and Hernandez Rivera, 2017). It was not until that following April of 2015 that university administrators held a town hall to present the "Progress of the Call to Action List;" by then, students' level of frustration grew from a lack of transparency and accountability from leadership and all but one of the central organizers had graduated (McElderry and Hernandez Rivera, 2017).

\section{The Case: September-October 2015 Incidents}

In September of 2015, the president of the Student Government Association, Payton Head, who identifies as a Black queer man, received racial and heterosexist slurs from people riding in the back of a pick-up truck (Pearson, 2015; McElderry and Hernandez Rivera, 2017; Rochester, 2019). On September $12^{\text {th }}$, Payton Head used Facebook to express his frustration from the incident stating, "For those of you who wonder why I'm always talking about the importance of inclusion and respect, it's because I've experienced moments like this multiple times at THIS university, making me not feel included here" (Pearson, 2015; McElderry and Hernandez Rivera, 2017). On September $17^{\text {th }}$ Mizzou's Chancellor, R. Bowen Loftin, acknowledged the "recent incidents of bias and discrimination," and stated they are "totally unacceptable" (Pearson, 2015). Unsatisfied with the university's response to address Payton Head's concerns, students protested on September $24^{\text {th }}$, followed by another demonstration on October $1^{\text {st }}$ where protesters chanted "White Silence is Violence, no justice no peace," during a second "Racism Lives Here" rally on campus (Pearson, 2015). During a practice for an upcoming performance on October $4^{\text {th }}$, members of the Legion of Black Collegians Homecoming Court were called the n-word by a drunken White man belonging to a historically White fraternity after interrupting their meeting and being asked to leave. Members of the group lamented that "not only did this individual disrupt our rehearsal, but we were also made victims of blatant racism in a space that we should be made to feel safe" (Pearson, 2015). Chancellor Loftin issued a statement the following day proclaiming that "racism was clearly alive at Mizzou, and that enough had not been done and that all community members must help [us] change [our] culture." (Pearson, 2015) Chancellor Loftin ordered diversity and inclusion training for students and faculty 
on October $8^{\text {th }}$ to begin in 2016; stating that the training would "inform all of us about the diversity of our campus and the organizations present on campus and make us conscious of how to be inclusive in our words and behaviors" (Pearson, 2015). As an acknowledgment to the chancellor's statement, on October $14^{\text {th }}$, student leader Johnathan Butler (2015) recognized it as a step in the right direction but disparaged the absence of mention of two areas. First, that the diversity and inclusion ideas for intervention were because of the work African American students, protesters, organizers, faculty and staff who used their time to hold the administration accountable. In short, this had not developed out of the administration's own volition. Second, that the intervention strategies were the result of the recent slur incident experienced by the Legion of Black Collegians rather than as a result of the historical pervasiveness of racism and hate that had been present since at least 1935 (Butler, 2015). On October $10^{\text {th }}$, protesters blocked UM President Tim Wolf's car during the Missouri Homecoming parade to address him directly regarding a lack of acknowledgement and response to the racial incidents on campus (Pearson, 2015; McElderry and Hernandez Rivera, 2017). Wolfe bumps a protester with his car in an attempt to avoid the blockade, and according to the student body president Head, Wolfe "smiled and laughed in their faces" (Pearson, 2015).

\section{Concerned Student 1950 Group's List of Demands:}

By October $20^{\text {th }}$ the student group Concerned Student 1950, named in honor of the year the first African American student was admitted to Mizzou, wrote a letter addressed to the University of Missouri outlining historical and recent pervasiveness of racism and discrimination and put forth their list of demands to administration. They are:

I. We demand that the University of Missouri System President, Tim Wolfe, writes a handwritten apology to the Concerned Student 1950 demonstrators and holds a press conference in the

Mizzou Student Center reading the letter. In the letter and at the press conference, Tim Wolfe 
must acknowledge his white male privilege, recognize that systems of oppression exist, and provide a verbal commitment to fulfilling Concerned Student 1950 demands. We want Tim Wolfe to admit to his gross negligence, allowing his driver to hit one of the demonstrators, consenting to the physical violence of bystanders, and lastly refusing to intervene when Columbia Police Department used excessive force with demonstrators.

II. We demand the immediate removal of Tim Wolfe as UM system president. After his removal, a new amendment to UM system policies must be established to have all future UM system president and Chancellor positions be selected by a collective of students, staff, and faculty of diverse backgrounds.

III. We demand that the University of Missouri meets the Legion of Black Collegians' demands that were presented in 1969 for the betterment of the black community.

IV. We demand that the University of Missouri creates and enforces comprehensive racial awareness and inclusion curriculum throughout all campus departments and units, mandatory for all students, faculty, staff, and administration. This curriculum must be vetted, maintained, and overseen by a board comprised of students, staff, and faculty of color.

V. We demand that by the academic year 20172018, the University of Missouri increases the percentage of black faculty and staff campus wide to $10 \%$.

VI. We demand that the University of Missouri composes a strategic 10-year plan by May 1, 2016 that will increase retention rates for marginalized students, sustain diversity curriculum and training, and promote a more safe and inclusive campus (Concerned Student 1950, 2015). 
VII. We demand that the University of Missouri increases funding and resources for the University of Missouri Counseling Center for the purpose of hiring additional mental health professionals; particularly those of color, boosting mental health outreach and programming across campus, increasing campus wide awareness and visibility of the counseling center, and reducing lengthy wait times for prospective clients.

VIII. We demand that the University of Missouri increases funding, resources, and personnel for the social justice centers on campus for the purpose of hiring additional professionals, particularly those of color, boosting outreach and programming across campus, and increasing campus wide awareness and visibility. (Kovacs, 2015)

After another incident on October $24^{\text {th }}$ where someone used feces to draw a swastika on a residence hall wall, the system president Tim Wolfe met with Concerned Student 1950 members privately but did not agree to meet any of their demands (Pearson, 2015).

\section{The Case: November Incidents}

Graduate student Jonathan Butler shared with fellow members of Concerned Student 1950 that he would begin a hunger strike the next day on November 3, 2015 (Pearson, 2015; McElderry and Hernandez Rivera, 2017). In the two-page letter demanding the removal of system president Tim Wolfe, Butler stated:

In the past 90 days alone, we have seen the MSA (Missouri Students Association) President Payton Head being called the n-word on campus, graduate students being robbed of their health insurance, Planned Parenthood services being stripped from campus, \#ConcernedStudent1950 peaceful demonstrators being threatened with pepper spray, and a matter of days ago a vile and disgusting act of hatred where a MU student drew a swastika in the Gateway residential hall with their own feces (Butler, 2015). 
Immediately, students coordinated efforts to camp out on the campus quad until the president resigned to save their friend [Jonathan Butler] (McElderry and Hernandez Rivera, 2017). Several days following the start of the hunger strike, Wolfe, issued an apology to Concerned Student 1950 on November $6^{\text {th }}$ proclaiming that "Racism does exist at our university and it is unacceptable. It is a long-standing, systemic problem which daily affects our family of students, faculty and staff" (Pearson, 2015). However, when Wolfe was questioned that night by protesters on his knowledge of "systemic oppression," his response that "systematic oppression is because you don't believe that you have the equal opportunity for success," angered protesters who asked him if he had just blamed them for systemic oppression (Pearson, 2015). On November $8^{\text {th }}$ Black players of the Mizzou football team, who were supported by their fellow White teammates, as well as the Athletic Department and Head Coach, Gary Pinkel, announced they would not play another game until Wolfe was removed from his post. This move garnered wide national coverage, and it was a move that could have cost the university upwards of five million dollars in game forfeitures (Pearson, 2015; McElderry and Hernandez Rivera, 2017). On November $9^{\text {th }}$, The Missouri Students Association's executive cabinet claimed that the system's administration failed students and called for Wolfe's resignation; hours later after 7 days of the hunger strike and 48 hours after the football team strike, the President of the University of Missouri System, Tim Wolfe, resigned (Pearson, 2015; McElderry and Hernandez Rivera, 2017). The University of Missouri-Columbia's chancellor, Loftin, resigned as well, after receiving a vote of no-confidence from the campus deans (McElderry and Hernandez Rivera, 2017; Rochester, 2019). Following the resignations of the president and chancellor, student protesters gathered in the university quadrangle with an assistant professor of mass communications, Melissa Click, who was on camera yelling at student journalists to get out, asking for "some muscle" to remove them and grabbing their cameras for trying to interview and film protesters (Rochester, 2019). Moreover, a Mizzou law professor and deputy chancellor emeritus was named interim head of the UM system; a month later a chief diversity officer was hired with a starting salary of $\$ 235,000$. As a result, a new three credit hour 
course (diversity intensive) was required for graduation; the coursed focused on "understanding different social groups" (Rochester, 2096). In like manner, Rochester (2019) shared that a UM system Diversity, Equity, and Inclusion Task Force was created to examine the complexities of all issues. To this end, faculty and staff were urged to complete social justice/diversity training sessions to earn points in the university's "wellness" program and a new bureaucratic unit to centralize the investigations of reports of race, gender and other discrimination called Office of Civil Rights and Title IX was created at Mizzou (p. 43). Given the nature of events that occurred at Mizzou, Rochester (2019), who was a faculty member within the UM system, was not clear on how the swift reform efforts would address Mizzou's ranking by Heterodox Academy as "the worse school in the country for ideological diversity" or the 35 percent drop in freshman enrollment between 2015 and 2017 (Rochester, 2019). With such an extensive turn of racist and discriminatory events, I will examine the demands put forth by the students to better understand how said demands invoked social change among various human networks who experienced these racial tensions. Such racist behaviors and marginalizing of non-dominant groups are not isolated to this this campus, but rather reinforce the continued existence of pervasive discontinuities in social equities of the past on college campuses. What makes this case exceptional is that, while its race dynamics are historically pervasive, we have a unique opportunity to examine the aftermath of a case having occurred within the last five years; we can now explore the current campus racial climate by coming to understand the interventions used to create institutional reform as a result of student demands.

\section{Problem Statement}

In framing what is essentially a campus climate dilemma, we must consider the historical manifestations that helped to create the foundation of the racial dynamics plaguing higher education institutions. The social variances and lack of intergroup relations between White university officials and White students with Black students has stemmed from deeply rooted issues related to racial inequity, discrimination, the apprehension of White people to embrace or interact with Black students in study 
groups, and the presumption that Black students had nothing to contribute. (Davis, Dias-Bowie, Greenberg, Klukken, Pollio, Thomas and Thompson, 2004). Thus, this historical racist tension played out in the campus climate and ecological experiences of African Americans at PWIs nationwide, making their academic, social, and psychological worlds substantially different from their White counterparts (Pascarella and Terenzini, 1991). To adjust to the campus climate, Black students had to abandon their cultural roots and swiftly adhere to White cultural norms (Smith, 1981). In response to such a harsh climate, Smith (1981) explained how Black students felt the need to counteract feelings of alienation and loneliness by dining together as a group, residing together, or joining all-Black social groups, which were discouraged by university officials. Administrators advocated for the downplaying of race, thinking it was in the best interest of all students. This downplay on race perpetuated our understanding of marginalizing non-dominant groups in predominantly White spaces, because the value or contribution of their race was sidelined and not viewed as mattering. Currently, African American students and faculty-administrators are experiencing racism, causing students to protest discrimination and unwelcoming campus environments, which have led to a flurry of controversy over their demand for social justice. African American students and allies are finding it difficult to perform well academically and socially in spaces that are not intentionally advocating for a socially just and culturally competent campus constituency (Wright, 2016). Institutions can better serve their constituents by coming to understand the discontinuities in experiences as mediated by race, in effort to learn what is most desired and demanded by its underrepresented students; especially regarding college persistence (Tinto, 2016).

This study has been designed to explore the phenomenon of a racially tense campus climate at the University of Missouri, a Predominantly White Midwestern Institution. This institution was in the forefront of the media regarding student and athlete protests, leading to the resignation of senior level administrators. The students put forth eight demands to their administrators to begin remedying the problems felt by African American students. Said demands called for direct administrative or curricular 
changes; some of which happened almost immediately. In fact, included in this list of demands to their administration, was the creation and implementation of a required racial awareness and inclusion curriculum throughout all campus departments and units. Given their problems with race relations and the actions that followed, this case gained national attention. My own university administrators began discussing the ways in which my school was either being proactive in their approach to avoid a chilly campus climate or how they might be at risk for the next massive campus outcry to gain national attention. The uptick in campus unrest continues to present some university administrators / leaders with the challenge of responding to new and ongoing challenges and student demands; of which, some have little to no response at all (Mahler-Rogers, 2017; Ruff, 2016). To this end, it would be highly advantageous for students, faculty, staff, and administrators to consider what those involved in the Mizzou case experienced initially and focus on what resulted as interventions to the demands put forth. In this qualitative method single case study, I sought to understand the experiences of those involved in this exceptional case to come to understand more how student demands may have been addressed or implemented as interventions for an improved campus racial climate. Thus, the purpose of this study was to explore the perceptions of the institutional response to an exceptional campus racial climate issue at the University of Missouri and the process of formulating and participating in a diversity training course and a semester long course centered around race. Additionally, this dissertation sought to understand how participants perceived this curricular intervention to have addressed the discontinuities in race and racism on campus and contributed to organizational change, and institutional reform.

\section{Research questions:}

This research was guided by the following questions:

1. What are the perceptions of the process of formulating and participating in a curricular response to an exceptional campus racial climate issue at the University of Missouri Columbia? 
2. What are the perceptions of how this curricular response addressed discontinues in race and racism on campus and contributed to organizational change and institutional reform at the University of Missouri Columbia?

\section{Introduction to racist and racially inequitable campus climates}

The University of Missouri is not alone in the struggle for a more inclusive, diversified, or welcoming campus environment that intentionally serves its African American constituents and create opportunities for shared learning and cultural awareness. Throughout our history, many institutions have experienced racially invoked campus unrest, which led to campus protests. I attempt to briefly address Black students being marginalized and demanding racial equity through campus protests and various reform efforts. Research on the experiences of Black students at Predominantly White Institutions (PWIs) have spanned almost 40 years, a foundational study by Smith (1981) found that "inadequate financial assistance, lack of supportive services, deficient skills, high attrition rates, and underrepresentation in undergraduate, graduate and professional programs are factors which inhibit the higher education advancement of Black people" (Smith, 1981, p. 300). Historically, students desiring to enter onto a college/university's campus received court ordered assistance from the national government, but still had to fight and protest their way into White schools with the help of the military. Once entered, the tense race relations between students and faculty-administrators did not dissipate, but rather attached themselves to the ways in which Black students would come to experience the campus climate and ecology. The following two sections highlight additional foundational cases at Kent State and Northern Kentucky to illustrate how the events in Missouri are part of a broader national phenomenon.

\section{Kent State University Protests}

Kent State university in Kent, Ohio is a foundational example of campus racial unrest. In the late 1960s, Black students literally packed their suitcases, marched through campus, and walked off campus 
through the front gate in protest. According to Dr. David A. Ambler, a former Vice President for Student Affairs, the students initial protest was ignited by the presence of Oakland California Police Department; they were invited to campus to conduct interviews for potential new hires. Because this was seen by Black students as a racist police department, they were disgruntled by their visit to campus and blocked the entrance way to where interviews were to be held. When university officials threatened to bring disciplinary action against Black students for violating university regulations, they organized their plan to, yet again, protest this decision. Students and one faculty member traveled to a neighboring college town, Akron, Ohio, and vowed to stay at a local Black church until their demands were met. Specifically, they demanded that charges against them be dropped, the employment of more minority faculty and staff, the creation of a minority affairs unit and developing of an African studies program. In doing so, this gained a significant amount of public attention; the spotlight was on Kent State University and officials felt pressured. The president agreed not to press charges and to establish a commission to investigate the minority student concerns. Ambler, the Associate Dean of Student at the time, shared that the Dean of Student's gave the students much more than what they asked for. If they asked for "A," he gave them "A," "B," and "C." To this end, Kent State opened one of the nation's first multicultural affairs offices (Formerly human relations), and later a Black Cultural Center (Wold-Wendel, Twombly, Nemeth Tutle, Ward and Gatson, 2004). For decades, Kent State has served as a model for Black students and faculty-administrators across the nation who desired to change the racial climate in similar ways; after all, this was where the Black United Students movement (Also known as Black Student Union) began. Although great outcomes resulted from the student protests and demands, there are a few important factors to note. Foremost, new needs, demands and strategies to create supportive campus environments are likely to surface with new advancements in knowledge, intergroup relations experiences and with new generations of students. To be clear, Northern Kentucky University, another PWI, sought to intentionally serve the needs of its Black students via targeted programming. However, this was stymied with some backlash from White students. 


\section{Northern Kentucky University Protest}

In 2016, some White students at Northern Kentucky University (NKU) posted a flyer to mock a new university initiative for students of color during welcome week called Welcome Black Week by posting flyers that read "Welcome White Week." Immediately, students gathered in protest of what they considered to be outright racism and a lack of understanding on the part of their counterparts for why such an intentional initiative was developed at NKU (Anstead and Grecco, 2016; NKU Students Respond to Racially Charged Flier for "White Week," 2016). The students who posted the flyer in response to the new initiative appeared unaware that others, who are different from them, exist with varying needs stemming from pervasive oppression due to their privileged positions. Having an environment that does not serve students using a one size fits all approach but rather seeks to create safe and welcoming spaces for all students, based upon the unique and intentional needs of various populations, could have proven useful to disgruntled White students. In fact, Hurtado et al.'s, (1998) campus climate framework espouses the usefulness and necessity for institutions to serve students with intentionality. Having access to the rich accounts of what all students experience and how to bring forth change in social equity among todays' generation would be valuable to gain and inform current or future practices.

While some may think racial marginalization is a thing of the past or that we now live in a postracial society, where race should not be used to examine our practices, African American constituents at Predominantly white Institutions may differ in their opinion. An uptick in such racist behaviors and racial inequities in environments where African Americans attend school has led to a considerable amount of campus unrest and discontinuities in student, faculty, and administrator experiences. While there are many racist incidents on university campuses we can reference, we are limited in our ability to gain from research a full understanding of the perceptions and responses of those involved in the implementation of demands as interventions. This study sought to understand the ecological systems and interactions of 
those involved, to learn how and why they worked to create reforms, centered around race to improve their environments.

\section{Conceptual Framework}

Critical Race Theory (CRT) evolved from the legal field's 1970s movement Critical Legal Studies (CLS), a leftist legal movement that challenged traditional legal scholarship that dealt with doctrinal and policy analysis of a type of law focusing specifically on the social and cultural contexts of individuals and groups. Cornel West (1993) asserted that CLS seems to call out what was wrong with the legitimacy and authority of pedagogical strategies in law school rather than what a new and legal society would look like. To this end, West was lamenting the frustrations of a scholarship that brings to the surface ideals that may cause us to pause for thought and awareness but have no clear plan to invoke change. CLS, in its attempt to critique the laws pertaining to social and cultural groups and portraying the US society as a meritocracy, failed to include racism in its critique. As a result, disgruntled legal scholars of color gave shape to Critical Race Theory. Thus, Critical Race Theory developed, as an outgrowth to CLS in its own category of scholarship, with a focus on race and racism and more advanced desired outcomes than what CLS sought to offer.

Critical Race Theory has three central propositions:

1. Race continues to be a significant factor in determining inequity in the United States.

2. U.S. society is based on property rights.

3. The intersection of race and property creates an analytic tool through which we can understand social (and, consequently, school) inequity. (Ladson-Billings and Tate's, 1995, p.48)

CRT has been used in both evaluation research and/or studies regarding the identity and intersectionality of underserved populations using either mixed, qualitative, or quantitative research methods. CRT's conceptual power is heightened when combined with theories of intersectionality. 
Crenshaw, Gotanda, Peller, and Thomas (1995) worked to combine feminist legal theory with CRT to uncover-as it pertains to law and popular discourse-the disempowerment on race lines and gender. To be specific, Crenshaw (1991) explored the race and gender dimensions of violence against women of color because contemporary feminist and antiracist discourses failed to view the intersections of racism and patriarchy. To this end, Crenshaw's (1991) focus on male violence against women considered "how the experiences of women of color are frequently the product of intersecting patterns of racism and sexism...Because of their intersectional identity as both women and people of color...the interests and experiences of women of color are frequently marginalized within both" (p. 1243-1244). Parker and Lynn (2002) expound upon the importance of an intersectional view by concluding that, although race and gender epistemologies have attempted to bifurcate and thereby essentialize identity into frozen fixed frames, an intersectional analysis forces us to see the relationship between sexism and racism as symbiotic. In other words, racism sustains and rearticulates sexism" (p. 12). As a result, Parker and Lynn (2002) assert that race does not exist outside of gender and gender does not exist outside of race. Moreover, given the inequities related to race, class and gender throughout our nation's history, Watkins Liu (2017) describes how CRT and Intersectionality combine to form the CRT/I lens. The combination maintains an integration of analysis pertaining to race, power, lived experiences, social location, and structures as a socio-structural model and to analyze the books in the field of social movement scholarship in her study; to be referenced in the analysis section of this dissertation. Thus, intersectionality, according to Watkins Liu (2017), adds to CRT assumptions - of which I previously discussed earlier-(1) intersecting categories (How different social categories interact/co-constitute), (2) multi-level analysis (How multiple levels of society inform and interact impacting lived experiences on social structure and vice versa), (3) power dynamics (Intersectionality examines how power operates in different social contexts) , (4) reflexivity (The constant act of critical self-awareness and reflection), (5) time and space (The inference that social phenomenon and dynamics must be considered within temporal and spatial contexts), and (6) 
diverse knowledges (The careful consideration of how marginalized groups are positioned within notions of power and knowledge production). Analytically, as a lens, CRT/I:

1. Identifies relevant social categories

2. Pays attention to the social construction of such categories-especially the historical context within which such categories operate

3. Is attentive to how power operates and is challenged

4. Operates at the individual level to investigate how nuanced lived experiences inform social structures and vice versa

5. Pays attention to the treatment of vulnerable voices, perspectives, experiences, and presentations

6. Analyzes the power within and the implications of knowledge production.

Consequently, these scopes set the backdrop for socio-structural analysis of power within scholarship, lived experiences, and society; all while grounding race as a foundation with the inclusion of additional social categories (Watkins Liu, 2017, p.3). Additionally, Parker (2004) advocated for the integration of evaluation and CRT by using it as a research lens in educational evaluation to analyze racial inequality and to see how organizational structures are developing, to give voice and empowerment to those who have been underserved by secondary and postsecondary institutions. Parker's (2004) study concluded that a CRT methodology and critical race policy analysis for education can be used to both frame the research issues that will be studied and interpret what emerges with a lens focusing on racial equity implications. According to Parker (2004), the use of CRT narratives was considered to elicit powerful counter-stories, of which can be an effective strategy in challenging myths that otherwise paint African American and Latino and Latina families as lacking in care or desire for their children to have a high-quality education. The suggestion from Parker (2004) is that this type of framework paring gives evaluators varying ways of looking at recurring policy patterns in school leadership and perhaps how that effects the educational 
equity of Black students. CRT's outward-growth from the critical legal studies to its centrality on race and racism approach gives voice to underserved populations through critical narratives and counterstorytelling that share their experiences in ways that are not often considered. Such experiences and critical analyzing of educational policies, programs, and structures will bring to the surface the explicit or implicit methods of inequity and oppression that the dissertator considers to be forms of systemic or institutionalized racism. CRT and intersectionality together, allows for multiple social categories to be examined within and among each other where they do, in fact, intersect. By avoiding the male-female polarization, we can see how oppression is entangled where race, gender and class collide. For that purpose, Delgado Bernal (2013) contend that “...these systems of knowledge, or critical raced-gendered epistemologies, emerge from the experiences a person of color might have at the intersection of racism, sexism, classism, and other oppressions" (p. 391). But this is not the end goal of CRT or intersectionality. These frameworks - even when used in evaluation research-seek to initiate changes in equity with the intent to force actions that would counteract traditional dominant power structures. My attempt, in this section thus far, was to briefly articulate the utility of various critical theoretical frameworks and their ability to be combined when necessary, to guide my study or analyze data.

In this study, I considered the propositions of CRT and or CRT/I where appropriate. CRT does not lend itself to a lock step or well-designed visual model/graphic to be followed, but rather is a conception of researched ideologies that use race as a focal point of inquiry in spaces and policies to test for the presence of oppressive forces that need to be remedied. A CRT/CRT/I lens was used to guide the research design and methodology. It was used to formulate semi-structured interview protocols for the students, faculty, and administrators and to analyze various policies and any document analysis that surfaced. As I sought to uncover the outcomes of the student demands put forth to the administration, it was done so by examining the action taken by the administration to understand the ways in which the voices of the underserved may or may not have been heard. CRT and CRT/I inquiry was useful to this study because it 
used race and intersecting social constructs to investigate spaces, policies, and practices; bringing to surface institutionalized racism and existing power structures that exist. Additionally, Critical Race Theory helped to examine how and why I found social inequities, to bring about change or improved practices. Thus, because I sought to focus on a campus knowingly plagued by issues of racism, employing frameworks that have been designed to examine such spaces was fitting.

Because CRT and CRT/I is not a lock step framework with concreate steps, stages, or systems, I used this as an overarching framework given its emphasis on race throughout the research process. The second framework used was the Human Ecological Systems Theory derived from Urie Bronfenbrenner. The theory was brought to light in the 1970's and has undergone some iterations to maximize its utility in research. Neal and Neal (2013) have offered a new way of understanding Bronfenbrenner's interpretation of the ecological systems as nested within one another. On the contrary, they argue for the systems to be viewed as networked within and between each other. This study used Neal and Neal's reiteration of Bronfenbrenner's works, while maintaining his foundational theory overall. The Human Ecological Systems Theory was traditionally designed to aid us in our understanding of how children develop because of their environments that are each influenced by different interactions with humans or various policies and beliefs that indirectly influence the child. The foundation of this theory offered the four-core system with one additional system, the chronosystem, to surface several years later (see figure 1). To be more in line with recent studies, figure 1. will also include the addition of a Techo-subsystem, referenced by Johnson and Puplampu (2008), to factor in the use of various forms of technology on an individual. A simplified description of these systems and Neal and Neal's adaptations are listed and described as follows:

1. Microsystem (nested within the Mesosystem)

a. Nested: The focal individual directly plays a role, has experiences, has social interactions with others. (e.g. as a child or sibling, enjoying meals, reading to a sibling or being read to.)

b. Networked: A setting-or set of people engaged in social interaction-that includes the focal individual (Neal and Neal, 2013, p. 728). (e.g. the focal individual can be engaged in social interaction with three different microsystems that overlap.) 
2. Mesosystem (nested within the exosystem)

a. Nested: The social interaction between members of the child's home and school microsystem. (e.g. a parent and teacher meeting about the child's behavior.)

b. Networked: A social interaction between participants in different settings that both include the focal individual (Neal and Neal, 2013, p. 728). (e.g. The relationship from persons from one microsystem creates a mesosystemic relationship with others from another microsystem, while both including the focal individual.)

3. Exosystem (nested within the macrosystem)

a. Nested: The setting that may influence or impact the child and or the environmental experiences without his/her direct participation. (e.g. an education policy or school building closing that can change the scope of the curriculum or classroom size)

b. Networked: A setting-or set of people engaged in social interaction-that does not include, but whose participants interact directly or indirectly with, the focal individual (Neal and Neal, 2013, p. 728). (e.g. This might be one setting without the focal individual where a set of individuals are engaged, but the interactions may vary as some individuals may be directly or indirectly connected to the focal individual.)

4. Macrosystem

a. Nested: The broader setting where overarching ideologies and federal policies can determine the way the focal individual experiences something in another setting. (e.g. a federal education act that dictates the way schooling will be experienced or beliefs about the value of education.)

b. Networked: Different from the micro, meso, and exo systems, this is the set of social patterns that govern the formation and dissolution of social interactions between individuals, and thus the relationship among ecological systems (Neal and Neal, 2013, p. 729). (e.g. Those who share the same race, gender etc. [homophily] or commitment to social justice form a set of social patterns that determine how social networks are structured. These social networks influence the kind of social interaction to occur in the ecological environments whereby the focal individual will experience.)

5. Chronosystem

a. Nested: The system reflecting the changes and continuity across all other systems over time. Transitions or occurrences that take place at a time that can alter or influence the focal child's experiences (e.g. the onset of puberty or transitioning from one school to another)

b. Networked: Also set aside from the first three systems, this is the observation that patterns of social interactions between individuals change over time, and that such changes impact the focal individual, both directly and by altering the configuration of ecological systems surrounding him/her (Neal and Neal, 2013, p. 729). 


\section{Figure 1}

Bronfenbrenner's Bioecological Systems model with added Techo-subsystem

Bronfenbrenner's Bioecological Model of Human Development

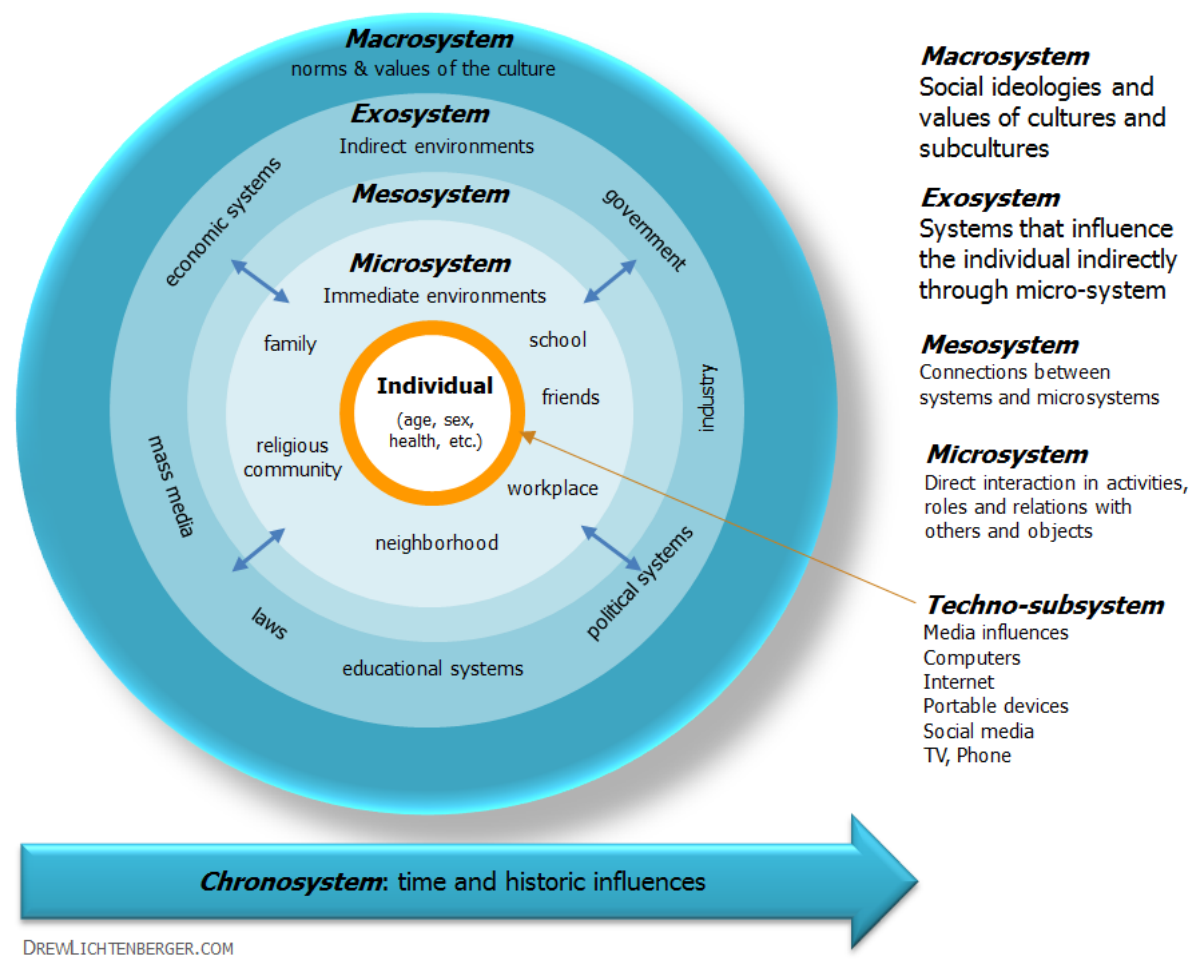

Note. The figure above depicts an example of an individual in the center of an ecosystem, whereby they are being influenced by the various systems that impact their development, resulting from a variety of spaces and institutions within each system. Johnson \& Puplampu, 2008, retrieved from

\section{http://drewlichtenberger.com/6-shaping-influences-human-development/}

As referenced earlier, Neal and Neal (2013) expound on this theory by proposing each of the first three systems as having the capacity to overlap with each other. Additionally, they asserted that there might be multiple environments that are a part of the same system; causing the focal individual to be influenced by the different social interactions taking place in - for example-three different microsystems. This theoretical framework helped to guide the study, as I was interested in the perceptions of students, faculty, and administrators at the University of Missouri. I sought to uncover the phenomena of student demands being 
used as interventions and what emerged from the various social interactions occurring within the systems aiding in the development of the focal individual(s). I utilized this framework with a CRT/CRT/I lens to place special emphasis on the networking of social interactions as mediated by race. See figure 2, from Neal and Neal (2013) for a visual display of the adaptation to Bronfenbrenner's Ecological Systems Theory by Neal and Neal. To analyze data, I situated aspects of what emerged, after a CRT/CRT/I lens was used to interpret data and create codes, into the most fitting of the six systems and reported how they were networked within and between systems.

\section{Figure 2}

\section{Networked Model of Ecological Systems, Focused on Person A.}

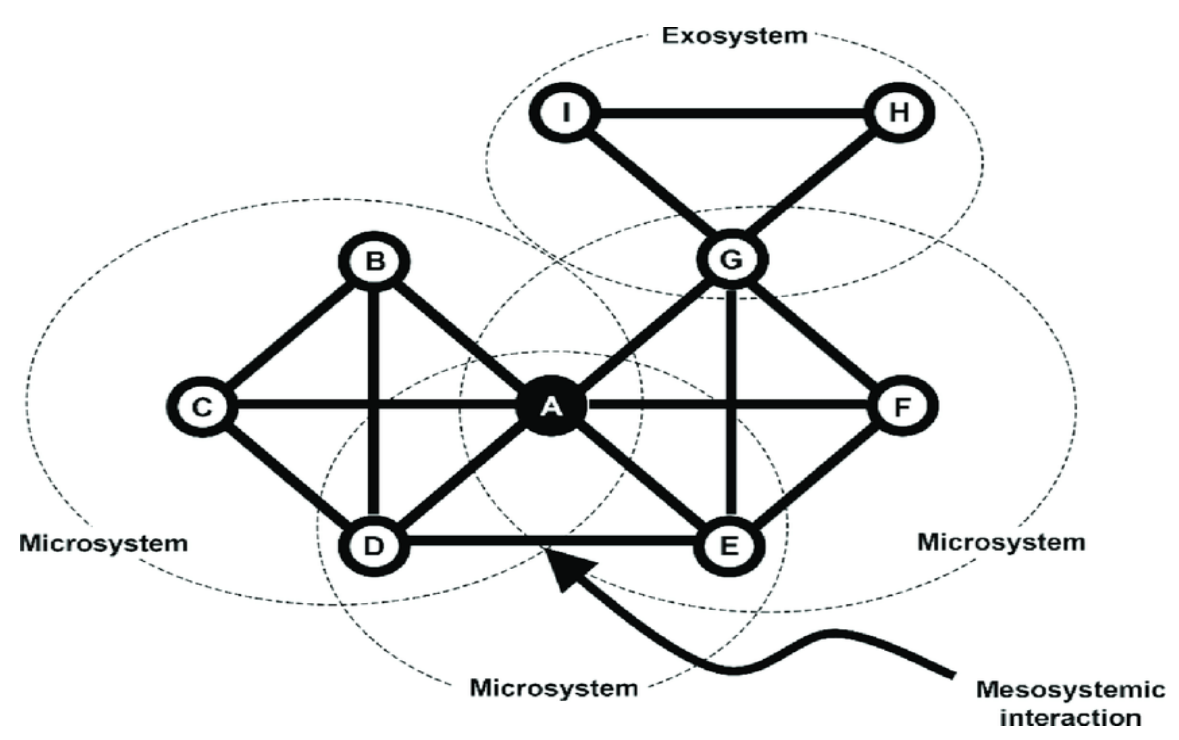

Note. A visual display of the adaptation to Bronfenbrenner's Ecological Systems Theory by Neal and Neal (2013). 


\section{Practical Significance}

Therefore, institutions must make it a priority to ensure they hear the voices of those on their campus who are often less heard, especially in spaces where they are underrepresented. Hearing these voices and understanding their needs, desires and demands afford practitioners the awareness necessary to intentionally create and design spaces, programs, services, and opportunities that will effectively aid their holistic development to become globally aware citizens. Student affairs educators and faculty may benefit from this study, as it uncovered the perceptions and responses of those involved in the racial tensions and or the reform efforts at the University of Missouri following the events. Additionally, students and practitioners may find it useful to gain knowledge on the ways in which these tensions were mediated because of student advocacy for social justice, through campus protests and demands. Higher education practitioners and faculty across the nation would be able to have an increased awareness of current trends in student desires and demands for a more comfortable campus climate.

\section{Scholarly Significance}

There is significant research on racial campus climate as well as broad coverage in academic and popular literature regarding the events at the University of Missouri-Columbia. However, this study contributes to the literature by addressing a gap in our understanding of the aftermath of campus racial events and the institutional process of responding to campus climate issues. In addition, this study addresses an understudied area of divergent participant perceptions and experiences in addressing climate issues after campus unrest, specifically by exploring how student's demands were implemented as institutional interventions, and by understanding the perceptions and experiences of building a required racial awareness and inclusion curriculum in the aftermath of such a destabilizing event. 


\section{Definitions}

- Chilly climate: Racialized spaces that devalue, marginalize and hinder the full participation of underrepresented or traditionally subordinated groups.

- (Human) Ecology: a branch of sociology dealing especially with the spatial and temporal interrelationships between humans and their economic, social, and political organization

- Epistemology: The study or a theory of the nature and grounds of knowledge especially with reference to its limits and validity. This is the way we determine justified beliefs from pure opinion.

- Inequity: A lack of fairness or justice.

- Intervention: The act of interfering with the outcome or course especially of a condition or process (as to prevent harm or improve functioning)

- Meritocracy: an elite group of people whose progress is based on ability and talent rather than on class privilege or wealth. a system in which such persons are rewarded and advanced.

- Predominantly White institution-PWI: A post-secondary institution where the enrollment of White students is at least $50 \%$ or greater of the total enrolled. 


\section{Chapter Two}

\section{Review of literature}

Much of the campus unrest happening across the nation at institutions of higher learning is motivated by institutional racism. To properly address current discontinuities of race among campus constituents, it is critical to consult the literature for the structural and historical manifestations that have prompted the continued forms of structuralized racism influencing patterns in human interaction and policy. While this dissertation will not provide the exhaustive historical backdrop for the evolution of institutional racism, given the limited scope and direction of this study, here is a brief overview to better understand why or how race has been used over time to form oppressive structures. These structures are often undergirded by policies, laws and-in some cases-no policy at all.

In addition to understanding what students demand from their colleges/universities, it is important to consider the antecedents of this activism. Within the literature, exists a wealth of knowledge on what served as the catalyst for Black students to become activists for campus climate change. With a Critical lens toward research with race and racism at the center, the literature discovered in this study brings to surface the antecedents and the current notion of "student demands," at PWIs. Consequently, this includes campus racial climate literature and how some institutions have responded to creating inclusive climates through various theories or interventions. Furthermore, the dissertation includes literature to understand campus reform and how various organizational structures might mitigate, exacerbate, or leave problems with the human ecological system unchanged.

\section{Brief Overview of Institutional Racism}

Institutional racism was first introduced by US Black political activists Stokely Carmichael and Charles V. Hamilton in their writings found in Black Power, published in 1968. Carmichael and Hamilton were quoted in Jones (1974) saying: 
Racism is both overt and covert. It takes two, closely related forms: individual whites acting against individual blacks and acts by the total white community against the black community. We call these individual racism and institutional racism. The first consists of overt acts by the individuals, which cause death, injury or the violent destruction of property. This type can be reached by television cameras; it can frequently be observed in the process of commission. The second type is less overt, far from subtle, less identifiable in terms of specific individuals committing the acts. But it is no less destructive of human life. The second type originates in the operations of established and respected forces in the society, and thus receives far less public condemnation than the first type (p. 218).

To summarize this idea from Carmichael and Hamilton, according to Jones (1974), institutional racism is "the operating policies, properties, and functions of an on-going system of normative patterns which serve to subjugate, oppress, and force dependence of individuals or groups by (1) establishing and sanctioning unequal goals, objectives, and priorities for blacks and whites, and (2) sanctioning inequality in status as well as in access to goods and services" (p, 219).

In chapter one [page 11], the dissertator presented three central prepositions of which LadsonBillings (1995) based their work on CRT. The purpose of revisiting the scholarship on CRT now, is-in partto show formative examples of structural/institutionalized racism. Because the focus of this dissertation is not to delve into the intricacies of each proposition, they are briefly summarized, but considerable attention is given to the second and third propositions that connect to education and the ways in which CRT posits itself and shines light on notions of institutional racism. Omni and Winant infer that the thought of race solely as an ideological construct, denies the reality of a racialized society and the impact of it on the everyday lives of raced people. When race is objectified, it denies the problematic components of race, such as: deciding who fits into which racial classification or the categorization of racial mixtures. Oddly enough, Ladson-Billings and Tate (1995) inferred that, because human beings group themselves in an array 
of ways, the category of race in biology is somewhat useless. However, whether the concept of race makes sense or not, it is still employed. Toni Morrison thought of race as a metaphor-a way to disguise forces, events, classes and expressions of social decay and economic division. Thus, Ladson-Billings and Tate (1995) argued that, unlike gender and class, which had [in the 1990s] a rise in literature production, race was untheorized. Rather, race was conflated with ethnicity, class, and nationality. While gender and class certainly matter and can yield useful data when researched, scholars believed that the two alone cannot provide a fuller understanding of all inquiries - particularly as it pertains to student outcomes and disciplinary records in school. Henceforth, there is a need for race to be examined in many facets of our societal structures, including property rights.

Ladson Billings and Tate (1995) argued that traditional approaches of the Civil Rights Movement depended on the "rightness" of democracy, ignoring capitalism as a structural inequality. The researchers also note that despite civil rights leader's social justice efforts that appealed to civil and human rights, they neglected the fact that the society was built on property rights, which is the second preposition in their work. In framing this idea, Ladson and Billings reminded us that tribes of Indians inhabited land in New England when Pilgrims arrived; however, the government of the time stated that the Indians only had a "natural" right, but not a "civil" right to the land, because they had not "subdued" the land. Thus, the area was declared legally a vacuum, giving the Indians no legal standing despite their "natural" right. Likewise, if we evaluate/examine the constitution and consider the presence of African peoples as slaves in America, it was clear that the purpose of the government was to protect the property of owners. Slaves, including women and children were objectified as property-lacking any form of human or civil rights. To this end, it may seem far-fetched to compare Blacks in America to property, but when the reification of Black individuals is to be symbolic recipients of equal rights, the centrality of property is more likely to be disguised. The researchers focused more intently on the individual and their rights, they also described how there are still benefits to property owners, especially in education. 
Ladson Billings and Tate (1995) help us to understand how this notion of race and property impact education, especially for raced people. To be clear, the literature describes the reality that those from more affluent communities pay a higher property tax. They resent paying for a school system primarily consisting of non-White and poor citizens; thus, those with better property are afforded better schools. Furthermore, curriculum is seen as a form of "intellectual property," whereby, its quality and quantity vary based upon property values of the school. For example, in a "Critical Race Story" major differences in course offerings were discovered between two students entering high school. The student entering an upper-middle class White community had a considerable amount of variety in foreign language, math, science and electives, while the student entering an urban and largely African American district was found to have limited options, but unfortunately lacked traditional electives such as band, orchestra and a school newspaper. Given this curricular shortfall, evident in the lack of "rich" or "enriched" intellectual property, it impedes upon the opportunity to learn, despite the presumption that students must have the necessary material resources to support the desired educational standards. Standards of which determine what students know or should be able to do. The researchers proclaim that "real" property must undergird intellectual property; including science labs, computers and state-of-the-art technology. Anything less would place students at a disadvantage; hence, the ever-present notion of educational inequity (Ladson Billings and Tate, 1995).

Nevertheless, this dissertation discusses Ladson Billings and Tate's (1995) third proposition that the intersection of race and property creates an analytic tool through which we come to understand social inequity. Harris (1993) contends, stating that due to the long-lasting victimization of people of colorparticularly as property-it made room for the construction of whiteness as the ultimate property; possessing the power to lay the foundation of rights in property based upon the cultural practices of Whites. She refers to four ideas that exemplify this notion of Whiteness as property: (1) rights of disposition, (2) rights to use and enjoyment, (3) reputation and status property, and (4) the absolute right 
to exclude. First, she states that although property rights are to be alienable (transferable), entitlements; professional degrees/licensures have limited transferability, given who they are held or controlled by. Thus, when Whiteness is conferred on certain student performances, it can be alienable. White property becomes alienable only when students are rewarded for conforming to traditional "White norms" and sanctioned for cultural practices (e.g. dress, speech patterns, unauthorized conceptions of knowledge). Second, Harris (1993) validates the claims of a previous scholar when she asserts that Whiteness allows for extensive use of school property - such premium space that can create an enjoyable learning atmosphere with the privilege to adhere to appropriate capacity specifications, unlike those in more poverty stricken and non-White areas. Additionally, Kozol (1991) claimed that the curriculum is, yet, another area where enjoyment may occur for the dominant population, but not be structured in such a way that would allow Black students to enjoy. Third, urban schools-which have come to mean Black-lack the reputation or status of White (suburban) schools and are thus, seen as inferior; the busing of urban students to these schools, can essentially be viewed as a strain on the reputation of the suburban school. The fourth and final idea is concerned with the notion of White flight from certain schools and communities, whereby they seek vouchers or public funding for private schools. The absolute right to exclude is displayed through new ways of segregation that might include gifted/honors programs and advanced placement courses. As one can see, the history of Critical Race Theory runs deep, both historically (informally and formally) and within our educational structures. This is what we have come to understand as forms of institutional or structural racism. Taking the time to closely examine, analyze or evaluate-using race and racism as a critical tenant to the theory - is important. For example, the educational spaces, policies and curriculum might lead to an increase in awareness about the opportunities for social justice reform, when applying a Critical Race Theory lens to various research studies and evaluation research that lead to advancements in program theory.

\section{How forms of Institutional Racism Surface in Higher Education}


Constant attacks, dis-respect, and mimicking blacks at the hand of whites via blackface minstrel shows at "fraternity parties, university-sponsored theater events and by the placing of racial and ethnic caricatures that ridiculed black students or blacks in general in class yearbooks; and the use of these caricatures to specifically ridicule African American Greek-letter organizations," (Breaux, 2012) depict the racial campus climate experiences over a century ago. As this mistreatment continued, more outrageous measures were taken to stifle the progress of African Americans known as lynching, which "became one of the most violent forms of expressions whites used to keep blacks "in their place." Between 1889 and 1932, over 2,785 blacks were lynched by whites in the United States" (Breaux, 2012). Such harsh measures were like that of vigilante justice; a way to discourage blacks from continuing to even think of pushing for social, political, or economic equality with whites; this perceived and observed success is what seemed to infuriate and exacerbate the attacks on black people everywhere, including higher education. In an article, "Nooses, Sheets, and Blackface: White Racial Anxiety and Black Student Presence at Six Midwest Flagship Universities, 1882-1937," in Perspectives on the History of Higher Education, Breaux's (2012) work used six Predominantly White Flagship Institutions (PWFUs) to provide the historical account-aforementioned-of a campus climate racked with racial tensions for underserved populations. In our attempt to discuss literature on campus racial climates and student experiences related to racial discourse/dialogue, microaggressions, institutional racism and campus ecology, we must consider the history that serves as a backdrop for our understanding of how or why this legacy influences the culture of our college/university campuses today. In fact, in a seminal work by Hurtado, Milem, Clayton-Pedersen, and Allen (1998), an institution's historical legacy of inclusion or exclusion of various racial/ethnic groups was one of four dimensions in the campus climate framework they developed. The other three dimensions include: structural diversity in terms of numerical representation of various racial/ethnic groups, the psychological climate of perceptions and attitudes between and among groups, and the behavioral climate dimension, informed by intergroup relations on campus (Hurtado, et al., 1998). 
To expound upon this seminal campus climate framework, the researchers make clear that these dimensions are not isolated from each other, but rather interconnected. The first dimension, the institutional context historical legacy of inclusion or exclusion, is built on the premise that racial climates (including diversity) is affected by the historical vestiges of segregated schools and colleges. Resulting in Predominantly White Institutions (PWIs) maintenance of old campus policies that benefit homogenous groups and attitudes and behaviors not conducive to cross-race interaction; sometimes without recognition of such inequity among groups (Hurtado et al., 1998). It is believed that "A college's historical legacy of exclusion can determine the prevailing climate and influence current practices (Hurtado, 1992). This is a strong assertion and places a heavy responsibility on institutions to act against the historical legacies of exclusion (oppression). Thus, an institutions initial response to creating a supportive environment by way of, but not limited to determining the philosophy of education for students of color, commitment to affirmative action, institutional intent for minority-specific programs, and providing attention to the psychological climate and intergroup relations on campus (Peterson et al., 1978). Of importance to note, Hurtado et al. (1998) declare that, while colleges and universities cannot change or should not deny their legacies of exclusion, taking intentional steps to place diversity at the center of their educational enterprise is possible. It cannot be assumed that students enter knowing these histories or that they will be dissatisfied with learning about or engaging with them. Second, structural diversity and its impact on students relates to the impact that racial/ethnic diversity or the lack thereof can have on the education benefits for students. Hurtado, Dey, \& Tevińo (1994) argue that students' learning experiences and interactions with socially and culturally diverse groups is limited when campuses have high proportions of White students; therefore, increasing structural diversity is considered an important step in improving campus climate (Hurtado et al., 1998). Moreover, with increases in diverse student enrollment comes the need for the development of ethnic studies programs, diverse student organizations, targeted academic support programs, and multicultural programming (Muńoz, 1989; Peterson er al., 1978; Trevińo, 
1992). In validating this claim, Hurtado et. al. (1998) summarized Chang's (1996) idea by having stated that “...increasing only the structural diversity of an institution without considering the influence of each of the other dimensions of the campus racial climate is likely to produce problems for students at these institutions" (p. 286). The third dimension of the campus climate framework is the Psychological dimension of climate and its impact on students. Individuals' views of the campus' racial climate are at the center here, as it pertains to: relations with various racial/ethnic groups, institutional responses to diversity, perceptions of racial conflicts and discrimination and their attitudes toward people from other racial/ethnic backgrounds. It would be no surprise that racially diverse administrators, students, and faculty would be found to have varying views of the campus climate (Hurtado et al., 1998). Furthermore, Collins (1986) asserted that depending upon ones' status as an "insider" or "outsider" and power within an organization, he/she can strongly influence attitudes. Here, is where it becomes important to consider how students are made to feel within the campus environment before they leave without ever reporting their negative experiences as a result of feeling high levels of alienation. The fourth and final part of this model is the behavioral dimension of climate and its impact on students. Three factors to consider here are (a) actual reports of general social interaction, (b) interaction between and among individuals from different racial/ethnic backgrounds, and (c) the nature of intergroup relations on campus. Many scholars conclude that student involvement is critical to undergraduates' successful education experience because it enhances their cognitive and affective outcomes (Hurtado et al., 1998). Globetti, Globetti, Brown, \& Smith (1993) that White students were less likely to hold positive attitudes towards multiculturalism on campus for those who had the least social interaction with others of a different racial background. On the contrary, those White students who experienced social interactions discussing race-related issues with non-white peers or attended diversity (racial) awareness workshops, were more likely to value the promotion of racial understanding (Milem, 1992, 1994, 1998). Thus, creating opportunities for intergroup relations dialogue to occur and student organization involvement with those from other racial groups, 
affords students an opportunity to begin engaging with aspects of identity development; this would prove to be beneficial for a more comfortable climate. Whereby, intentionally and proactively taking steps to rewrite the historical narrative in effort to avoid having a campus whose students lack awareness and concern for notions of social justice and the valuing of minority specific support initiatives.

Donald H. Smith (1981) produced an article more than 35 years ago entitled Social and Academic Environments of Black Students on White Campuses. This article helps us to understand the dynamics of racial tensions of that time period which further provide a context for the importance of our concern for how PWI's serve students of color. Smith (1981) enters the study with two questions: What is the effect of these environments on Black persistence toward graduation? And how do these environments affect the students' personal development? He claimed that "inadequate financial assistance, lack of supportive services, deficient skills, high attrition rates, and underrepresentation in undergraduate, graduate and professional programs are factors which inhibit the higher education advancement of Black people" (Smith, 1981, p. 300). This was the impetus for taking Critical approach to the social and academic environment Black students experienced while hoping to achieve an education and degree. Socially, Smith (1981) shares how Black students were viewed by White students and faculty as being special admits and beneficiaries of affirmative action. Consistent with this finding is Bennett and Sekaquaptewa (2014) account of women and racial ethnic minorities in engineering majors. They noted that prevalent stereotypes exist for these groups regarding their lack of success and ability in engineering; as a result, they become targets of discrimination in subtle and overt ways. Thus, majority group majors may hold negative attitudes towards diversifying the field and question whether women and racial/ethnic minorities even belong in engineering. This may cause underrepresented populations to leave the field of engineering altogether or impede upon their ability to thrive academically or feel comfortable within their own identity. Likewise, Steele, Spencer, \& Aronson (2002) explained that such occurrences mightpsychologically - trigger a perceived threat to the self (social identity threat) which is felt when people feel 
devalued in a given setting because of their social identity. This threat is believed to have the ability to activate the negative stereotypes, which may result in low-performance and an increase in anxiety. Traditionally, in order to adjust to the campus climate, Black students had to, perhaps, abandon their cultural roots and swiftly adhere to White cultural norms. In response to such a harsh climate, Smith (1981) explained how Black students felt the need to counteract feelings of alienation and loneliness by dining together as a group, residing together, or joining all-Black social groups, which were sadly discouraged by university officials. In fact, one dean of students proclaimed that "in the interest of all students, race must be downplayed" (Smith, 1981, 301). Fast forward for a second to 2016 and consider the comparison between the "Black Lives Matter" movement vs. the counter phrase "all lives matter." When "all" lives are not being threatened or targeted the same way Black lives are, the focus on race is being downplayed despite the pervasive presence of race that drives our intergroup relations or the lack thereof. During the 1960's and 70's it was Black students, again which were being targeted and abused, not "all" students, but administrators had a problem with targeting the necessary support services to aid Black students in their development. Smith (1981) considered this to be impeding upon the very activities which could protect them and increase their likelihood to remain in college. Other literature consistent with these claims are described in McCabe's (2009) work on “Racial and Gender Microaggressions on a Predominantly-White Campus: Experiences of Black, Latina/o and White Undergraduates."

In this multi-methodological study, framed under Critical Race Theory, she examined racial and gender patterns in microaggressions that Black, Latino and White women and men dealt with on a PWI. To this end, the four themes that emerged were: (a) views of Black men as threatening, (b) views of Latina as sexually available and exotic, (c) the classroom as a particular setting for microaggressions experienced by Black women, and (d) male-dominated academic majors as particular settings for microaggressions experiences by White women (McCabe, 2009). First, several scholars provide this definition for a microaggression: it "refers to the more subtle and covert acts, often identified as verbal or nonverbal 
insults. Microaggressions are brief, 'subtle and stunning' encounters that are a frequent occurrence in the lives of subordinated groups and that impact views of the self" (Delgado \& Stefancic, 2001; Pierce, 1970:266; Solórzano et al. 200; Sue et al., 2007). McCabe (2009) discovered in her findings that all students in the study reported having felt discomfort and isolation in the college environment. Additionally, many of the students described their experience as a culture shock when structural diversity-a dimension of the campus climate framework aforementioned-was lacking, it heightened some students' awareness about racial issues but also led them to feel isolated as outsiders on their campus. Furthermore, Black men in this study described how they were viewed as a threat to White people on their campuses if they wore a doo rag on their head; the feeling is that White people become scared of them or think they are dangerous. To reiterate, these instances and feelings are consistent with earlier claims in the literature referenced by other scholars. Moreover, Latina women and men of color of often experienced anonymous harassment in their residence halls without any idea who left signs of racial or gender offensive slurs or physical evidence; making these students feel even more paranoid and uncomfortable. Of note, Black women in this study lamented about their range of microaggressions in the classroom; especially being the "token" Black person having to bear the burden of representation for all Black people every time their professor calls them out to address issues related to race and minorities. Considering all the accounts that emerged from the aforementioned studies, it would be of importance to consider the ways in which an institution might more intently focus on minority student success as a way to foster a better campus racial climate and ecology.

\section{Toward a Better Campus Racial Climate from Institutional Racism in Higher Education}

To begin framing this idea, note that campus ecology was introduced to the student affairs profession by James Banning and Leland Kaiser (Banning \& Kaiser, 1974) and is "the study of the relationship between the student and the campus environment...incorporates the influence of environments on students and student on environments" (Banning, 1978, p. 4). Barker's (1968) behavior- 
setting theory is built on the premise that environments are behavior settings, whereby behavior can be predicted. Thus, it was believed that if student affairs educators know enough about students, environments and the behaviors that those environments will elicit, they might be able to predict student behaviors and take the necessary steps to manage the setting to produce different behaviors (Evans et al, 2010). Moos's $(1973,1979)$ social ecological approach suggests that environments, like people, have unique personalities. Ones perception of campus climate will impact their behavior in that environment; furthermore, this includes student living situations and classroom settings. With the notion of campus ecology in mind, we can see how at least these two approaches appear to be consistent with similar ideas described in the literature throughout this paper. To conclude, the dissertation will present a study from a Generating Ethnic Minority Success (GEMS) institution which has some interesting ideas to consider.

This study used the "Campus Cultural Framework for Minority Student Success" which was constructed using the literature reviewed in the study. The four components include: campus culture, campus cultural agents, cultural connections, and racial and ethnic minority students' success. The framework is helpful when examining the campus cultures that may influence racial and ethnic minority students' connections to the campus and thereafter. It, for this study, looks at the values, beliefs and assumptions of the cultures of the GEMS institutions. Museus (2011) found it necessary to present Kuh \& Whitt's (1998) definition of campus culture as the "collective, mutually shaping patterns of norms, values, practices, beliefs, and assumptions that guide the behavior of individuals and groups in higher education and provide a frame of reference within which to interpret the meaning of events and actions"(pp.12-13). This notion, coupled with prior research (Attinasi, 1989; Gonzalez, 2003; Guiffrida, 2006; Khu \& Love, 2000; Museus, 2008a, 2008c; Museus \& Harris, 2010; Museus \& Quaye, 2009; Rendon, Jalomo, \& Nora, 2000; Tierney, 1992, 1999) that indicates students of color are more likely to succeed when they can connect to these cultures, helps to hone in on the uniqueness in responsibility of an institution as it pertains to ensuring their campus climate is conducive to forging connections from the onset of ones collegiate 
career. This would include culturally relevant institutional programs and practices which address student's cultural heritage. Museus (2011) points out that according to several sources (Allen, 1992; Feagin, Vera \&Imani, 1996; Lewis, Chesler, \& Forman, 200; Park, 2009; Khu \& Love, 2000; Museus \& Quaye, 2009) countless students of color often experience complications trying to connect with cultures of their PWIs. To this end, Museus and Quaye (2009) compiled an intercultural perspective of racial and ethnic minority college student persistence by using the voices of 30 students of color at PWIs that emerged from existing literature. This perspective suggests "that students of color who originate from cultures that are incongruent with those on their campuses can experience cultural dissonance, which can be defined as the tension that occurs from incongruence between a student's home and campus cultures (Museus, 2008a). The author goes on to assert that such dissonance makes it difficult for students of color to be influenced towards success, given the lack of connection with the culture of the campus. It is believed that such a disconnect causes feelings of alienation, marginalization and of being unwelcome in the campus culture (Allen, 1992; Feagin, Vera \&Imani, 1996; Lewis, Chesler, \& Forman, 200; Park, 2009).

Overall, the campus racial climate is of critical importance to consider for higher education practitioners and constituents. There are so many factors of concern for why it is important to even make this a priority. The historical legacies of a campus' climate or culture is of considerable importance to the future trajectory of an institution's racial campus climate and should be a driving force in determining how the school will move from traditional forms of racialized oppression experienced in old policies and practices. The campus climate framework and the campus cultural framework, along with familiarity of the theoretical foundations of campus ecology, would be beneficial to ensuring a more comfortable and culturally aware campus climate where intergroup relations and student involvement are encouraged, but also financially supported by institutions. In fact, McCabe (2009) shared an implication for educational policy-a tenet of Critical Race Theory-in that results of her study suggest that institutions should continue and increase their support to student organizations. The reason, they helped students to create a 
sense of belonging with others who supported their racial and gender identities while transitioning to college; several students were disappointed by the demise of racially-based groups they found to be of help to their transition and success. Overtime, the chilly conditions of many college campuses has sparked the need for a paradigm shift for race relations and learning; leading to Black student protests and their demands as interventions for an improved campus climate.

\section{General Overview of Campus Racial Climate \& the Journey to Creating Inclusive Climates}

Chapter one introduced the historical pervasiveness of racism and oppression experienced by Black people over time and the struggles fought by student activists as a backdrop to the need for student demands. This study, is by no means, attempting or claiming to have included all student protests or campus unrest issues, which can be tracked as far back as the late 1800s; to do so, would diminish our goal of examining current trends as mediated by race, demands and interventions. However, in addition to those instances referenced, this dissertation delves a bit deeper into the heterogeneity of resistance that show a correlation to the foundational power dynamics/inequities that campuses are facing today. During the 2014-2015 academic year, 61 protests of the 160 student activism incidents were sparked by issues of racism and police violence (Johnston, 2014). To support this claim, the Southern Poverty law Center (2017) has reported an increase in hate groups in society, but particularly on campuses, from 892 in 2015 to 917 in 2016. According to the Anti-Defamation League ( $A D L)$, white supremacists have been vigorously targeting U.S. college campuses since January 2016. 161 incidents on 122 different campuses in 33 state and the District of Columbia were documented; from September 1, 2018 to May 31, 2019, 313 cases of white supremacist propaganda on campus were documented (ADL, 2019).

Ndemanu (2017) concluded that these hate groups' actions, which have been intended to terrorize and intimidate students based on race, are being tracked by newspapers around the country. Ellis (2017), prepared a list of vandalism, frightening posters and spray-painted swastika's among others. Specifically, a 
University of Texas poster that read "around blacks, never relax," while another read "Imagine a Muslimfree America" were among this list. Some scholars see the \#BlackLivesMatter movement, after a series of police shootings and murders of unarmed and non-threatening Black people, as the fuel to ignite the mobilization of Black students to demand systemic changes in and around their PWIs (Wilson, 2015). According to Gusa (2010) and Patel (2015), they note that it is the traditional race neutral ideologies and discourses which mask the unequal distribution of power in institutions and society. Likewise, Hamer \& Lang (2015) emphasizes the issues with said anti-racist discourse by claiming that it "absolves predominantly White universities of any responsibility in substantively altering institutional policies and decision-making, effectively leaving the burden of racism to people of color" (p.889). In other words, some institutional policies and discourse exist or are uttered to "cover" themselves in the event of a racism crisis - like an insurance policy-rather than make clear proactive and ongoing actions to ensure equity is realized and felt among non-dominant groups and is favorably upheld by dominant groups and those in power. Thus, this forces underrepresented students to call out the lack of intentionality and commitment from campus officials to make good on policies and discourse available for public consumption; whereby, driving the conceptualization of student demands. It is these experiences and processes this study hopes to examine more closely, coupled with those of faculty and administrators (Faculty-administrators) from the University of Missouri (Mizzou), which is the single exceptional case for this study. Solórzano \& Yosso (2016) support this idea of experiential knowledge from people of color as it is considered "legitimate, appropriate, and critical to understanding, analyzing, and teaching about racial subordination" (p.129). In other words, claims like this found in the literature, coupled with our understanding of CRT/I help to validate our focus on students' demands to help us uncover useful data for those who seek empirical knowledge as a framework to guide institutional change efforts or to improve the function of the human ecological system. Thus, exploring Black student demands allows us to reach more accurate conclusions about campus racial climate, the human ecological system, of which include faculty-administrators, and 
the types of interventions that may be elicited from said demands to address change the organizational structure of institutions.

To further examine the intricacies of Black student demands, Ndemanu (2017) analyzed the Black students' demands nationally across 73 colleges and universities in the United States, which he did to "lay bare the multifaceted layers of institutionalized racism which have contributed to the current and recurrent racial tensions in the various citadels of learning" (p.238). Using CRT, he does an in-depth analysis of students' demands and "some of the most egregious racist policies of the past," that he believes contributed to today's "untenable racial climate in higher education" (p. 238). Ndemanu (2017) uses a qualitative research method, document analysis, as the only method of data collection given the vast availability of newspaper articles, press releases, offensive pictures/posters/graffiti and a website, thedemands.org, which compiled the demands of the 73 colleges and universities analyzed in this study. The intellectuals responsible for the website's organization of content are known as We The Protestors, a group of national collaborative activists fighting to end racism and police violence in America. Of importance to note, the demands on the website currently have expanded to 80 colleges and universities from the past several years. From the documents analyzed, Ndemanu (2017) found the following demands to be most prevalent across institutions:

1. Increasing diversity in the professoriate

2. Increasing the percentage of minority student enrollment

3. Requiring cultural sensitivity training for faculty and staff

4. Requiring a racial or social justice course in the core curriculum for all students

5. Divestment from certain corporations

6. creating safe space (e.g. Black culture centers)

7. De-naming and renaming historical buildings and schools across campuses.

8. Asking university leadership to issue a statement or apology about incidents of intolerance and oppression 
Using specified key phrases, the researcher used key phrases in the coding of news articles and college websites; priority was given to the themes considered most salient for university officials to address to minimize similar or re-emerging scenarios across institutions. Constant comparative method (Glaser \& Strauss, 1967) became the undergirding method used to classify the codes into clusters and next themes, as similarities and recurrent concepts emerged (Ndemanu, 2017). Next, using the data from the 73 universities and colleges across the nation, Ndemanu (2017) was able to determine the frequency of various demands presented to school officials depicted in Figure 3.

\section{Figure 3}

\section{Colleges and universities across the United States ranked in order of frequency}

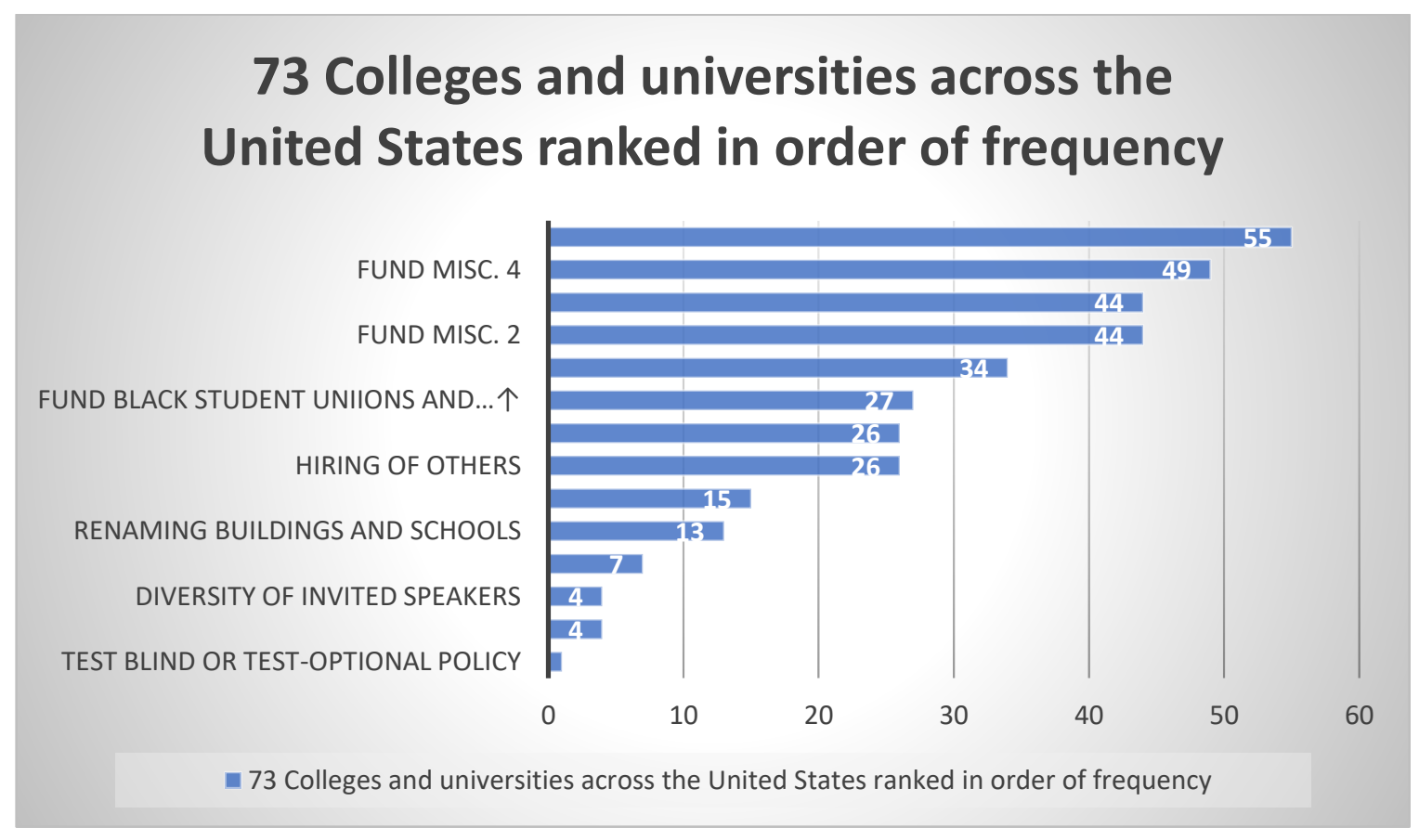

Note. (Ndemanu, 2017, The Journal of Negro Education, 2017, Vol. 86. No. 3)

In reference to this study, due to space limitations, the categories of demands in this article and figure 3. are not an exhaustive list of demands and are somewhat general in nature and limit our ability to see subcategories or the intricacies of demands, which fall under-in some cases-broad categories. However, the 
information provided helps us to see how widespread some demands are across the nation, given the amount of times they surfaced on student demand documents analyzed in the study. While it is extremely helpful to capture the dominant demands of Black students, for a change in campus climate, across the nation, this dissertation study must delve deeper to understand the empirical accounts of these students within the human ecological system.

In synthesizing the literature reviewed for our study, I find it necessary to see how other studies validate or differ from the findings presented in one study to the next. To this end, several instances were found in the literature that support the demands in Ndemanu's study by providing experiential knowledge from Black students who give us access to their lived experiences. For example, Jones and Reddick (2017) designed a single site instrumental case study (Stake, 1995) using focus groups for non-student leaders in the Black community and semi-structured interviews (Lincoln \& Guba, 1985) to interview multiple highly involved Black students holding leadership positions in both Black student organizations and traditional white organizations at a southern flagship PWI. In doing so, participants of the study were able to shine light on the power dynamics and challenges faced on their journey toward achieving lasting change within the ecology of their PWI. The researchers settled on the following themes to situate the data that emerged from participants; they are: the dichotomy of black student leadership, the emotional cost of individual and collective action, symbolic versus structural support, and disillusion of diversity efforts. (Jones \& Reddick, 2017). Although these themes are not overtly the same as what was found in Ndemanu's study, the content from student narratives provide context relatable to the broad demands. To compare, Figure 3 of Ndemanu's (2017) study list creating a safe space as one of the reoccurring students' demands; Jones and Reddick's (2017) findings contains a Black student leader's narrative relating to safe space within student leadership. To be clear, participants expressed significant value in serving the Black community as leaders of Black organizations as a way to form "shelters," within the campus environment, to emphasize and carryout the interests of Black students' needs to collectively unite against feelings of marginality in 
the broader context. Museus (2008) validates this notion, as their findings indicated that Black student organizations have been defended as a source of shelter at PWIs against marginalization within the dominant campus structure. But in addition to their involvement in Black organizations, participants placed considerable value in undertaking leadership roles in traditional White student organizations underrepresented by Black students. For example, one of the participants in Jones and Reddick's (2017) study expressed it this way:

If you graduate and don't connect to student government, you don't connect to these power players. I hate saying this. once you've been a president of [Black student organization], there's no more growth for you in those organizations...you have to move into student government or something else but stay connected. You have to get your foot in something else, in order for you to grow and make the best of the university (p.209).

This, forgoing counter-story helps us to understand the dichotomy of black student leadership and the limited ability of these organizations - on behalf of their community - to fully impact individuals and the university space as a whole. In other words, as described by Jones and Reddick (2017), participants realized the unjust benefits white organizations received linked to traditions and administrative support. Likewise, they discovered how Black representation in traditional white group spaces could yield a capacity for power to the individual leaders, but also a collective power for the Black community. But participants [Black leaders who networked with traditional White groups] did not find this easy to do; they had to accept the "tokenism" perception in taking these roles, while grappling with the pushback from the Black community, who often viewed their involvement in white organization spaces as personal gain. In part, because these "token" Black leaders in white spaces were often sought out by administrators/the media to address nationally debated topics [e.g. affirmative action) and how they affected the Black community (Jones \& Reddick, 2017). Jones and Reddick (2017) compared this dilemma to the "Talented Tenth" philosophy, in that an elite assembly of Black leaders attained individual prestige, which uplift the masses 
(Allen, 1998). Another participant in the study explained that because their graduate student council did not address discriminatory issues experienced by students of color, Black students seldom networked in this space. Because of students self-selecting not to participate-in what was viewed as White space-it stymied their ability to impact policy. On the other hand, when Black students vied for a place within student government association, they were often unsuccessful because they were not plugged into the White Greek system to secure adequate funding to yield a successful campaign to victory. Consequently, it reinforced their position as the lowest of the racial hierarchy; therefore, participants expressed having to be strategic in the parts of campus [college of Natural sciences] where there was a heavy concentration of Black students to rally together around Black candidates to get a seat at the table (Jones \& Reddick, 2017). Whether Black students led Black student organizations or participated in traditional white student organizations, the support they received from administrators [Channels of power] was appreciated, but the leaders who built these networks to produce benefits for the collective Black community began to question the longevity of said supports beyond their tenure. This leads us to better understand the nuances and motivations behind the widespread demand for institutions to support Black Student unions and the likes of entities that intentionally serve as safe social and academic spaces for students of color. It is clear from the findings in the literature-as expressed by participants - what the space feels like for Black students and how its traditional white spaces appear oppressive and structurally exclusionary against people of color. To this end, it provides us with an understanding of why the demand for safe space is important to them as Black leaders and to their collective Black communities.

As mentioned earlier, Ndemanu, (2017) points out the overwhelming instances where students across 73 institutions demanded their institution's support or funding of Black Student Union, multicultural centers and other structural supports. While the foregoing demand may be in a separate category among others, a demand for financial support to student organizations and initiatives connects to the idea of creating safe space. As this study continues the journey to understand this quandary, we must continue to 
consider the antecedents of the petition and the process by which this demand would be best implemented as an intervention. Participants in Jones and Reddick's (2017) study expressed the tension between those leaders who "worked in the system" and those who "overtly resisted" the system; it emphasized the need for heterogeneous approaches to Black leadership. Approaches, of which, included networking within the system [ecological in essence] to secure funding support from the "bureaucratic channels of power" (p.211). But to what end? Would these supports exist if it were not for the student leaders with select access to administrators? Or will funding supports continue when these token Black student leaders graduate? The answers to these questions were apparent in the actions of the leaders, as they found it critical to train the next generation of Black student organization members. One participant explained:

So it's like, "y'all know how to do this" in my absence. You know the things you shouldn't say-use target words and things of that nature...because the [the administration] thinks it's so much easier to just ask those same students...but it's like "I want y'all to be able to do it too," because we won't be here for long (p.211).

While the above participant, and others alike, used a special language to network with those in power and were successful in gaining financial access to benefit the collective, he also expressed how such approaches lessened or excluded other voices from the community to be heard. Administrative emphasis on funding raised concerns about the nature of symbolic giving vs. structural support.

Students in Jones and Reddick's (2017) study reported their experiences where university administrators gave emblematic endorsements through funding or promises for reform but failed to rattle the institution's structural inequities which remained unaddressed. Despite a racist "border party" held by a fraternity and a facebook page created in direct opposition to Black students' demands by peers, the students studied lamented their institution did nothing when incidents were off campus and gave no 
response in other cases. On the contrary, when the University of Oklahoma's president became aware of a racist video from an off-campus fraternity party, he severed ties with said fraternity because it went against the values of the institution. This immediate and clean break sent a clear message to all constituents what the latter university will not stand for or associate with as it pertains to racism and the mistreatment of its students. Again, those studying this literature are coming to understand some of the experiences behind the demands of Black students in Ndemanu's (2017) study through those studied in Jones and Reddick (2017), especially as it teases out what an actual safe space would look like. In other words, the conclusions that can be drawn from these studies are that administrators must do more than fund Black student unions and create multicultural centers; they must implement the structure within polices to elicit a campus climate where students do not feel as if their needs, desires and demands are being curtailed by monetary support but not true campus reform to the masses. In fact, one participant described her relationship with the president by stating " We have had those conversations, He has given funds to Black organizations, Black causes...with no policy changes...I hate to say this, they are throwing money into the community but not really solving the issues" (Jones and Reddick, 2017, p. 212). Moreover, also found in the literature instances where student participants in Jones and Reddick's (2017) study felt as though their administration relied on student-led efforts as opposed to institutional efforts to provide programming and support for Black students. Participants expressed concern that, while the Multicultural Center and New Student Assistance may have sponsored most efforts, they were led and staffed by students. On the contrary, another program called Friendship Camp received greater administrative support than the annual Black Student Weekend (BSW), which was only partly funded by the university. The bulk of the funding for BSW was achieved by student-led fundraising of up to $\$ 5,000$; this, left students feeling extremely taxed for organizing and fundraising. The disconnect between Black student programming and the administrator's absence in the institutional development of said programs, indicates a lack of understanding of Black students' needs and a lack of interest in creating a campus climate that is 
intentional about designing institutional structure geared towards serving Black students with specificity as it pertains to their unique needs to ensure holistic success for a population who requires a non-one-sizefits-all approach to student development. To support this assertion, a participant named Terence describes the experience for Black students this way: “I've been very involved in BSW and that is a completely student run initiative...I struggled a lot with that...getting it properly publicized [the weekend]. He stated that BSW was “a recruitment tool...I don't know if they're going to move on that...It felt like the university really honestly didn't care about really recruiting Black students" (Jones and Reddick, 217, p. 212). In addition to this program, was an orientation program called "Keeping it Real." The program was removed and re-introduced and enlarged to non-Black students, which Jones and Reddick (2017) stated created a gap for Black students hoping to foster community in that institutional control-in this manner-removed the notion of authentic discussion. Another participant, Courtney, lamented "Yeah, they eventually too it out ["Keeping it Real"]. Then my second year, we got it put back in, but we couldn't call it Keeping it Real...We also used to be able to stand at check-in and even if we weren't in the program, we would give them little papers that say what the Black events are that are going on. And then they told us we couldn't do that anymore (Jones and Reddick, 2017, p.212). The rational the Black students received for the abovementioned student-led intentional strategies, was that it might make White students feel uncomfortable. Likewise, a campus orientation social justice program was required for all students, but was later made optional because administration posed the question "why are students having to listen to diversity talks and be triggered?" Courtney's response to this rationale was "What's wrong with that one moment of un-comfortability, when we have four years where students never get over that "(Jones and Riddick, 2017, p. 213)? The student participants who took on the roles of organizing, advertising, fundraising, and executing programs they believed enhanced the campus racial climate and experiences for Black students, suffered from what Padilla (1994) denotes as cultural taxation. To this end, Jones and Reddick (2017) concluded participant experiences by explaining how the participants in their study felt 
they had a major responsibility to carryout intentional programs for Black students, while the administration remained uninformed about the valuable impact their work had on recruitment and retention. Additionally, the mere removal of intentional safe-guards [programs] and re-packaged versions of said programs to later serve all students or made optional, left participants believing that anticipated discomfort of White students were superior to the discomfort felt by Black students throughout their entire university experience (Jones and Reddick, 2017, p. 213).

\section{A Closer Look at what Undergirds Black Student Demands and Activism}

As this review continues, I sought to draw from the literature a clearer understanding of the racial climate across institutions and how racially tense campuses become the catalyst for Black student activism and demands. In doing so, Jones and Reddick (2017) have done a particularly good job of bringing to the surface the lived experiences of Black students attending PWIs and how the actions or polices of their administrators and institutions are cultivating an oppressive or marginalizing human ecological system. Such actions - or the lack thereof-and the absence of an institutional structure or policies aimed at nurturing Black student community needs and development, have a large influence on the demands put forth in Ndemanu's (2017) work. The previous study somewhat undergirds the latter by providing the why beneath each demand. For example, Ndemanu (2017) addresses the demand Increase Minority Faculty which was put forth by 55 of the 73 colleges in the study; the following demands were made: "We demand that by the academic year 2017-2018, the University of Missouri increases the percentage of Black faculty and staff campus-wide to 10\%" (Crawford, 2016) and Purdue University, "We demand that there be a 20 percent increase of underrepresented minority faculty and staff by the 2019-2020 school year" (Staff Reports, 2015). First, this demand for an increase in minority hiring is an umbrella statement for students - referenced in Jones and Reddick (2017) — wanting more institutional structure as it pertains to the carrying out of various university practices and the creation or modification of policies executed with the voices of non-dominant populations at the table. In fact, Ndemanu (2017) surmises this by sharing how 
such increases improve the chances of racial minority representation to help with or sit university committees where many policy decisions are made within department and college levels; ultimately affecting: faculty hiring, tenure promotion, curriculum and student recruitment and retention (p.244). The participants' issues with no structural and policy support could perhaps be addressed by increasing minority faculty and staff. But in addition to the connections between these two studies, Ndemanu (2017) expounds upon the importance of a campus that has higher percentages of minority/Black faculty, which could be why so many students across the nation list it as a demand. 84 percent of full-time faculty at fouryear degree granting colleges are White compared to 3\% each for Black and Hispanic faculty, even though the school-age (5-17) population rate for white students is decreasing while minority rates remain the same or are increasing. Said rates, are believed to foreshadow what will be seen in college, especially with more liberal admissions policies on the rise; therefore, it would be beneficial to have a more balanced faculty to avoid the domination of White professors over increased numbers of minority students (National Center for Education Statistics, 2013; NCES, 2013; Ndemanu, 2017). Likewise, Ndemanu (2017) states that a shortage of minority faculty prioritizes Eurocentric perspectives over others and limits the likelihood that Black students might aspire to join the ranks of academia, given a lack of relatable role models and mentors or the absence of exposure to Afrocentric scholarship. Prospective students and professors of color might be discouraged from applying to an institution for not having a human ecological system where underrepresented individuals can network and develop through shared experiences as raced people within a dominant campus population. To summarize this demand, professors have enormous power to produce and expand knowledge (Godin \& Gringas, 2000; Ndemanu, 2017), especially with tenure status. They can publish controversial works that address issues of power, oppression, racism and dominance without fear of being terminated. Thus, the researcher concludes this idea by proclaiming:

Therefore, if we are really determined to interrupt and decolonize the dominant narrative about the dominated and marginalized groups, the latter would have to be included in the 
knowledge expansion continuum; and this can only happen if more minority faculty members are being hired on tenure or tenure-tracked. Black students might have seen the difference it makes to not only take classes from Black faculty members on their campuses but to also read their scholarly articles. Expanding the number of faulty of color also benefits other faculty and students of European ancestry because it helps imbue them with new ways of knowing and expand their epistemological, cultural and linguistic horizons (Ndemanu, 2017; Hurtado, 2001, pp. 243-244).

Given the endless benefits described from the literature and the abovementioned quote, it is clearer to us why so many institutions of those studied for Black student demands, demand to see an increase in their faculty and staff. People of color in these roles can also be a source of knowledge for the entire campus constituency; particularly about a Campus-wide cultural sensitivity training for all faculty and a required racial/social justice course in the core curriculum.

Participants in Jones and Reddick's (2017) study lamented about their frustration in the university's decision not to require a social justice orientation program by making it optional. In line with this frustration, Ndemanu (2017) points out the overwhelming concern of students across the 73 institutions where students made demands along the lines of a required cultural awareness training and/or a required social justice/diversity course. In fact, the second highest demand across the board was for universities to require a campus-wide cultural sensitivity training for all faculty. The author points out a comparison that Pells (2013) made about 55 of the 69 Bowl Champion Series football universities requiring a mandatory sexual assault training and tightening their policies after the Penn State child sex-abuse scandal. He questions how many racist incidents need to be reported before universities do the same by requiring a cultural sensitivity training. A considerable number of expertise argue that given the uptick in colorblind philosophies and the overwhelmingly negative connotation of one being associated with racist ideologies or uncovering implicit or explicit biases, people are less likely to succumb themselves to such 
exposure and fear being labeled as a horrible human being. For one to self-reflect and acknowledge fault to themselves or others contrasts with a dominant group's early socialization experiences. (Marx, 2006; Alexander, 2010; Crenshaw, 1997; Ndemanu, 2017) Therefore, institutions that make said trainings optional, may be less effective in their efforts to provide critical education for awareness and development to change attitudes and learned belief systems among those of European descent. Thus, leaving a large and influential part of the campus population ill-equipped to navigate the nuances of cohabitating with non-dominant groups within the human ecological system. Today, researchers consider racism to be more passive than active and learning about the many forms of institutionalized racism will help people divest the prejudices they have internalized or exude implicitly (Marx, 2006; Delgado \& Stefancic, 2001;

Ndemanu, 2017). Similarly, the fourth most common demand from students was for universities to require a racial/social justice course in the core curriculum. While ethnic studies programs have expanded since the 1960s, the pre-college educational system has remained unapologetically Eurocentric and only deals with Black culture during Black history month in a limited, superficial or sometimes in contrast to Black scholars' beliefs about Black history. As a result, a large majority of students who enter college, do so with skewed versions and little to no knowledge about "American" history, which may include the harsh realities of treatment for minority groups (Zinn, 2003; Ndemanu, 2017). Additionally, I would also assert, from my own empirical knowledge, the shallow view of peoples of African descent only being viewed as slaves or inferior to the White race; neglecting the rich traditions and contributions of Black people on America and the world. To understand this as a campus climate dilemma, we must consider the ways academics influence cultural perspectives and behaviors of learners; who in turn, network and interact with the rest of the human ecological system, both in college and beyond. A larger concern becomes, how do we want institutions to effectively prepare all students and faculty for a diverse society to include social justice. Nedemanu (2017) points out that although institutions across the nation offer courses that align with a racial/social justice philosophy, they are not a part of the required core curriculum; therefore, 
students may graduate and enter the diverse workforce without having taken, at least, one racial/social justice course to prepare them. The researcher also asserts that while one social justice course is not enough to address racial issues in totality, it is a worthy first step for institutions to consider when trying to cultivate an inclusive and welcoming campus community for all. I suppose, a campus climate more conscious and intentional about how it serves underrepresented students might be better positioned to receive students of color into the campus community with less of a concern for campus unrest. To this point, the third most highly ranked demand by students in the study was to increase the enrollment of students of color.

The desire to recruit and retain more students of color was consistent with both Ndemanu (2017) and Jones and Reddick's (2017) studies. Earlier in this review of the literature, I came to know how participants in the latter study lamented about their concerns over having to take the lead on targeted programs and events for students of color; they saw these efforts as recruitment tools and strategies to help retain students. Along these lines, Ndemanu's (2017) study helps to understand the rationale behind this highly sought-after student demand. For example, in 2013 the six-year graduation rate of Black firsttime full-time students was 41 percent compared to 71 percent for their European American counterparts, which is the lowest among various racial groups (Musu-Gillette, Robinson, McFarland, KewalRamani, Zhang, Wilinson-Flicker, 2016). Another example described is predicated on the belief that European American pre-service teachers who may be inclined to gain more knowledge and understanding about race perspectives. He asserts having one or two students of color in class may limit the opportunity for a pedagogical practice that elicits multiple perspectives and that such enclosed spaces of the dominant group are breeding grounds for passive racism. Furthermore, White students in the Midwest have expressed only being exposed to racial difference during college; thus, robbing them of the opportunities to disrupt and address passive or active forms of racism in their psyche. (Ndemanu, 2017) Overall, the researcher references the claim that Intergroup Contact Theory has the ability to reduce prejudice 
between dominant and non-dominant groups when they are afforded opportunities for intergroup relations; whereby, increasing minority enrollment becomes necessary (Allport, 1954; Pettigrew \& Tropp, 2011). However important it may be to increase minority student enrollment, it cannot happen at any noteworthy rate if minority students cannot breakdown the barriers to enrollment. Barriers of which were brought to surface by only one institution in the study of student demands; that being a Test-optional or test-blind policy.

Black students at the University of North Carolina-Chapel Hill was the only institution to list a demand for their school to eliminate the consideration of SAT or ACT scores for the student admissions process. Their rationale was that “...these test correlate most closely with higher household income, disproportionately benefiting wealthier, White students. Following in the steps of Wake Forest University, UNC-Chapel Hill must become accessible to students who are presently marginalized from attending" (Ndemanu, 2017, p. 246; http://www.thedeamnds.org ). While this demand was an outlier of the study, Ndemanu (2017) asserted that there is a general propensity to view outliers as insignificant; however, he considered it to be one of the most transformative strategies to remedy minority enrollment inequities given the data reported from other institutions who theorized or implemented a test optional or test blind policy. For example, the percentage of Black students in Espenshade and Chung's (2011) study would have increased from 8.3 percent to 11.3 percent if standardized tests were optional and from 8.3 percent to 13.8 if tests were excluded. Additionally, Drew University noticed an increase in Black college student enrollment, from 12 to 54 students with their implementation of a test-optional policy and Black student enrollment increased by 35 and 41 percent, respectively, at Knox College and the College of the Holy Cross (Jaschik, 2006; Ndemanu, 2017). One concern for said changes in test policies is whether the student's academic performance or graduation rates would be up to par with the institution's rigorous expectations or suffer. But Bates College's test-optional policy showed that there was no difference in academic performance or graduation rates between those who submitted SAT scores and others who did not over a 
20-year study (Bates News, 2005). Likewise, Wake Forest University's website shares similar outcomes with their test-optional policy, as it states “...Furthermore, there has been no difference in academic achievement at Wake Forest between those who submitted scores and those who declined to do so..." (Wake Forest University, 2017). Although the results seem favorable and logical enough to elicit a reasonably swift improvement in minority enrollment, Ndemanu (2017) warned us of skeptics to the testoptional policy who believe that some universities might only implement said policy to swell their acceptance rate as more will apply and more will be rejected. The researcher points to what Critical Race Theorists refer to as Interest Convergence; in this context, the implication is that economic interest to boost rejection rates might be the ulterior motive for dominant groups to desire to embrace a testoptional policy rather than to benefit minority students. Similar skepticism has to do with the reputation of the institution and a fear of being viewed as less rigorous than other schools, but, according to this study, these arguments were debunked by U.S. News and World Report. (Delgado, 1995; Moss, 2017, Ndemanu, 2017) Either way, removing the standardized testing barriers to increase enrollment for minority students is even more logical to support, when other demands are being met; they provide the necessary safeguards and support structure to increase holistic achievement and student satisfaction with the overall campus racial climate experience. So far, a plethora of Black student demands across 70 institutions and the qualitative data that emerged to support or rationalize the campus climate circumstances that lead to prevalent and highly sought-after demands has been presented. A review of widespread student-demandliterature is the broad understanding needed to develop a foundation to better understand the single exceptional case being studied at The University of Missouri (Mizzou). A case, of which, has its own set of unique demands, much like those discussed in the studies above. What has been discovered thus far, are issues of human interactions, which are best understood through the lens of identity and how people grapple with their own journey towards racial identity development.

\section{Understanding Racial Identity Development for campus reform}


Our nation has been faced with numerous racial and social issues that have plagued the voices of non-dominant groups. As a result, there has been unwarranted deaths of Black men by police, mass murdering of LGBTQ+ people, overt hate responses and micro-aggressions expressed on our college campuses, and a current fear as to whether underrepresented groups will be safe in America with the current U.S. presidential administration. It is important for all people to understand their role in bridging this horrible and divisive divide impeding upon our ability to unite as a nation among and within cultures. For educators at all levels of education to effectively prepare students to engage across racial and social identities, they must provide strategic opportunities for their experiences to become interconnected. Students must understand, explore, and interpret their own life-worlds as well as the experiences of others in effort to construct individual meaning that would elicit a change in one's cognition, affective perspectives, and behavior as it pertains to diversity. Such notions led to the creation of a conceptual framework of Individual Diversity Development (IDD) by Chavez, Guido-DiBrito, and Mallory (2003). While this is technically considered a social development theory, there is much that can be derived from each dimension that describes, in general terms, the mindset, feelings and behaviors of which people-of any racial identity - are associated with. Given that our primary goal, aside from reviewing racial identity theories (e.g. African American, White, and Latino/s), is to discuss the research on curricular and cocurricular interventions that promote diversity, social justice, and racial identity development outcomes as demanded by students, IDD helps frame the rationale for this type of inquiry. Additionally, the descriptions of each dimension in IDD fit well with one type of curricular intervention in particular-intergroup dialogue-in our attempt to understand its intricacies related to the empirical influence on participants. Of note, the dissertation will discuss literature that has tested other interventions as a part of the aforementioned goal for this paper. Precisely, I explored studies that observed a dedicated diversity course vs. infusion in social work pedagogy; the comparative impacts of service learning, intergroup dialogue, and a diversity lecture-based course and the setting of an egalitarian social norm among engineering students. 
To properly frame the widespread lack of racial awareness and diversity/social justice dilemma for the context of this review, it is important to understand the negative implications that a lack of diversity development among college-age students will have on the collegiate environment. Furthermore, the dissertation study takes interest in how said development will influence future communities and work environments, of which these students will ultimately be a part of, upon exiting college. To this end, identity dualities surface and power structures, non-inclusive systemic practices and policies go unnoticed, unrecognized, or unconcerned about the continued oppression of non-dominant groups. This is an issue within various spaces across college campuses, including but not limited to: residence halls, student centers, Greek-life spaces, etc. Specifically, Torres, Howard-Hamilton \& Cooper (2003) spoke about the communication gap that leads to racial tensions in higher education even among faculty and administrators who lack knowledge about identity development. Thus, making institutions of higher education less equipped to deal with such conflicts dealing with race and ethnicity. To validate this claim, Hurtado (1996) conducted a study which found that one in four students at four-year colleges perceived "considerable racial conflict" on the campus, resulting in only 12 percent of college students at four-year institutions who thought racial discrimination was not an issue any longer in the United States (p. 492). Because of the racial tensions described from the literature in the previous sections about the camps unrest at institutions throughout the nation, it is known that traditionally marginalized students have protested or stood in solidarity with fellow institutions in order to advocate for inclusion and social justice. Unfortunately, this type of advocacy and concern for breaking down power structures that undermine equity for raced groups may be less likely to occur if people do not understand or consider notions of racial identity development.

\section{Curricular and Non-Curricular Reform Through Identity Development and Intergroup Dialogue}

There is a diversity learning gap among people from various racial/social identities that should be explored to better understand how these groups might learn and develop differently. It will enable 
practitioners to determine necessary future coursework in diversity education-along with other noncurricular interventions-unique to each group studied. More than 70 institutions in Ndemanu's (2017) study, including the University of Missouri in particular, demanded that a diversity education curriculum [racial/social justice course/diversity training] be implemented and required for its student and staff constituents. To explore this or similar ideas further, it would be important to measure the effectiveness of such efforts-in other instances - to see how the student's learning about racial/social diversity concepts are influenced. Studies have produced positive outcomes of-one type of curricular interventionIntergroup Dialogues to accomplish this task.

\section{A Brief Understanding of The Individual Diversity Development Framework}

For this paper, it is important to be aware that racial/social identities are being used somewhat interchangeably or in a closely related fashion. Racial identities are typically tied to identity theories that speak to a set of experiences, processes, stages, oppressions, privileges and ideologies of people, as it pertains to the race in which they ascribe to. Social identities expand upon this understanding to include other socially constructed identity theories such as gender, ability, and Christian ability; all of which can be explored with the aforementioned Individual Diversity Development framework (Zúñiga, Nagada and Sevig, 2002). Chavez, Guido-DiBrito, and Mallory (2003) have created this unique framework to gauge ones' process of development in the following areas: cognitive, affective, and behavioral when considering how people value the complex differences in others and themselves. This framework specifies six nonlinear dimensions in which developmental movement occurs, which include: 1.) Unawareness, 2.) dual awareness, 3.) questioning and self-exploration, 4.) and 5.) risk taking or other exploration, and 6.) integration and validations of others. A review of the literature discovered that in order to determine ones' achieved dimension across diversity as a whole, it is measured by looking at a person's a.) cognitive, b.) affective and c.) behavioral condition (Evans et. al, 2010; Chavez et al., 2003). To learn what is necessary to work through all dimensions, it requires further examination of each dimension in more depth to later 
discuss how the notion of Intergroup Dialogue can aid educators in helping students. First, Unawareness/lack of exposure to the other represents the place where people are when they have little to no knowledge or exposure with others who are unfamiliar. Cognitively, there is no consciousness or exposure to difference; affectively, there are no reference points of experience which yields a void of any type of feeling for otherness; behaviorally, the individual does not act when presented with differences. Next, Dualistic awareness is described by Chavez et al. (2003) in the following manner: cognitively people understand difference dichotomously without reflecting on it and thus, interpreting others from sudden analyses. Affectively, the egocentric self does not question their own analyses or expectations about "the other," making themselves superior. Behaviorally, the individual may have limited contact with others outside of their familiar groups, causing the person to correct others by pointing out what they perceive as incorrect behavior or by trying to exclude or eradicate others from the environment (Chavez et al., 2003). With this in mind, the behavior would be less likely to change if not presented with an opportunity or intervention-like Intergroup Dialogue-that could lead to changed feelings (affective) as a result of examining inaccurate information (cognitive). A further look at the literature showed similar value in measuring the cognitive and the affective positions of the learner; Nagda et. al (2014) mentions this when they discovered issues with the way some faculty had previously structured their diversity courses. Thus, they suggested to faculty that when teaching about diversity and oppression, it needs to address students' cognitive and affective development issues. They recommended utilizing both didactic and experiential learning while also motivating students towards both intellectual and emotional responses to course content (Nagda et. al, 2014). In response to these recommendations, Intergroup Dialogue is considered to be an ideal curricular-intervention strategy to intentionally aim toward achieving optimal outcomes. 


\section{Identity Development and Intergroup Relations Dialogue}

To understand the interconnectedness of the two models, Zúñiga, Nagada and Sevig (2002) state that Intergroup dialogues lead to student interaction and learning across differences by creating opportunities for student engagement with diversity (p. 7). So what is Intergroup Dialogue? IGD's are faceto-face sessions that promote evocative engagement between members of two or more social identity groups where potential conflict exists or who have a history of conflict (Zúñiga et, al, 2002, p. 232-248). The literature is clear in differentiating between what is discussed here and the other social identities that students may possess (e.g. athlete, an English major, and musician); the focus here deals with systems of power and privilege. Originally, in the 1940s and 1950s, the intergroup relations movement focused on reducing prejudice by shining light on the similarities among social groups in effort to bridge differences. Overtime, cross-cultural education was used to educate about culturally appropriate behaviors; all of which has led to how it has been used in more recent years, particularly the Program on Intergroup Relations at the University of Michigan referenced by Zúñiga, Lopez and Ford (2012); more on this later after exploring further research of the diversity development dimensions and specific racial identity theories (Zúñiga, Nagada and Sevig, 2002).

The third dimension is the questioning and self-exploration phase; the literature declares the critical relevance of this dimension in its depiction of how the individual begins to process feelings, opinions, and behaviors of others (Chavez et al., 2003). It is here where, cognitively, the individual begins to question their original belief systems (e.g., religion, familial, cultural practices) while affectively dealing with apprehension of being rejected by loved ones. However, at the same time, welcoming feelings of elation when contemplating the notion of opening the mind to a broader understanding of who they are, others, and the world itself. In line with this notion, the literature suggests that such constraints can be dealt with via dialogue. Dialogue, according to DeTurk (2007), has the potential to both underpin and contest these structures; its outcomes are unavoidably dialectical, recurring, and unpredictable, which 
highlights the natural risk of progress toward change. This could make people a tad uneasy or nervous. Additionally, Risk taking, or other exploration allows individuals to become engaged in the lifeworld of others; they move towards this notion of confrontation with their personal views and can even feel uncomfortable among family and friends whose values differ (Cognitive). At this point one can start to ponder their place in such a broad sea of diversity; (Affective) on the other hand, this pondering of feelings may lead to the act of advocacy for others by way of assisting them. As one seeks to become more aware of others and take risks to explore, I would argue that having a firm grasp on racial identity theories can be useful in coming to understand the intricate dynamics associated with such theories. Zúñiga, Lopez and Ford (2012) presented the interesting notion that intergroup dialogue cartels a critical examination and conversion of the oppressive social issues to elicit actions that would work to fulfill goals for a world where people can critically examine inequities, oppressive relationship dynamics among social groups that cause some to be advantaged over others, racism, sexism, heterosexism, etc. Such a critical examination coupled with dialogic praxis would give marginalized groups the capacity and opportunity to question and reject oppressive scripts and to name themselves, breaking free from the constraining and inaccurate narratives and dualisms. This powerful claim found in the literature characterizes the necessity for educators to structure their learning environments with opportunities for growth in diversity development. This idea is the epitome of what can be experienced via IGD in tandem with IDD and-for example-Black, White and Latino/a identity development theories, as it pertains to the previous two dimensions as well as the sixth and final one.

Likewise, the final dimension in this individual diversity development framework is integration/validation; this is the culmination of what we hope students can achieve and experience cognitively, affectively, and behaviorally. To expound upon this further, students who have reached this dimension can cognitively understand their civil rights, privileges, and responsibilities and those of others (Chavez et al., 2003). Differences and similarities between the self and others become recognizable, 
whereby the student reaches a level of comfort coupled with enough self-esteem to be secure in who they are in relation to others. As a result, this creates a culture of balance among their feelings, ideologies, and actions; this culture can permeate into the community, college campuses and work environments we wish to change. For example, Gurin-Sands, Nagda, \& Osuna (2012) found a positive correlation between students who were enrolled in a diversity course and social action engagement. An experiment of a dialogue and control group further validate this claim; a year after the dialogue group's treatment, they had a greater increase in commitments to action as compared to the control group that enrolled in social sciences courses on race and gender (Gurin-Sands et. al, 2012). To take this research further and discover best practices for improving higher education's campus climate and future community relations among racial and social groups, we must consider the ways in which we use these researched theories and practices to elicit positive change and discovery of self and others. For this purpose, I have provided an overview of racial identity development.

\section{Racial and Cultural Identity Development}

According to Evans et. al (2010), racial identity theories concentrate on the role of race and how it is incorporated into identity or self-concept. Helms (1993) defines the notion of racial identity as "a sense of group or collective identity based on one's perception that he or she shares a common racial heritage with a particular racial group" (р. 3). Given the inherent focus on race and ones' experiences with it and the associations with oppression, domination and privilege, Evans et. al. (2010) situates this idea in the context of Critical Race Theory (CRT); a model of which challenges and deconstructs the interaction of race, racism, and power. In other words, how people utilize CRT as a framework or lens of examination is connected-in many ways - to the place in which people find themselves being associated with their racial identity. And depending upon where one is with their own racial identity, CRT can help to validate and provide a rationale for the actions, in which, a person may take as described by their stage, dimension, status etc. Much of the literature on racial identity was built from the work of Erikson $(1950,1968)$, Marcia 
$(1966,1980)$ which led to the work of (Cross, 1978,1991; Helms, 1993a; Kim, 1981) and others. One model that helps to understand the stages and orientations that targeted racial identity theories use is the Racial and Cultural Identity Development (RCID) model by Sue and Sue (2003), which was originally developed as the Minority Identity Development Model by Atkinson, Morten, and Sue (1979, 1989, 1993, 1998). RCID is comprised of five stages: (1) Conformity, (Identifying with White culture, internalizing negative stereotypes about themselves/racial ethnic group and having no desire to learn about their cultural heritage.) (2) dissonance, (Experiences contradict ones' White worldview and initiates the desire to start questioning dominant culture and increasing knowledge of their own culture.) (3) resistance and immersion (The rejection of White Culture for the immersion into learning about themselves and their cultural group, leading to the formation of a new identity.) (4) introspection (The struggle of balancing between dominant culture and their own in order to shape identity.) (5) synergistic articulation and awareness (The acceptance of the new self, appreciation for the contributions of other groups while balancing their racial identity with other aspects of their identity) (Evans et. al, 2010, p. 254). In this regard, when comparing the literature on each of the racial identity theories, RCID can be used as a backdrop to guide our understanding of Black, White, and Latino/a identity theories.

Black Identity Development Theory. Evans et. al (2010) shared that other scholars including; Helms, 1990; Jackson, 2001; Sellers, Smith, Shelton, Rowley, \& Chavous, 1998 created models of Black identity development, but that Cross's theory of psychological nigrescence under Cross and FhagenSmith's model of Black Identity Development was best known. Nigrescence is the "process of becoming black" (Cross, 1991, p. 147) whereby three concepts: personal identity (Traits and characteristics that comprise an individual's personality.), reference group orientation (Personal values, how one sees the world and their philosophical or political lens guiding their views.) and race salience (The significance of race to ones' life approach.) were introduced. Given page constraints for this paper, this dissertation will 
not attempt to discuss the intricacies of all six sectors of Black Identity Development, but rather provide highlights; however, the sectors are listed below:

1. Sector One: Infancy and Childhood in Early Black Identity Development

2. Sector Two: Preadolescence

3. Sector Three: Adolescence

4. Sector Four: Early Adulthood

5. Sector Five: Adult Nigrescence

6. Sector Six: Nigrescence Recycling

Throughout each sector, the theory references the notion of one possessing either low or high race salience or internalized racism as developed from the parental socialization referenced in Sector two. For example, Cross and Fhagen-Smith (2001) described how they assumed most adolescents hold some awareness of a "Black self" which is accepted without critical reflection; also known as having a foreclosed identity. Additionally, to reach an achieved identity status, one enters a moratorium phase and realizes that their self-concept is based upon their own beliefs and not others. Those who enter a moratorium with low-race-salience or internalized racism patterns might be able to move beyond this and develop a healthy Black-self-concept with high race salience. On the other hand, these Black youth may confirm their self-concept of low race-salience, indicating that lack of exploration of Black identity for other preadolescent-learned concepts such as being American and not African American. Nevertheless, Black youth that possess an internalized racism identity pattern during adolescence (Sector three) will probably maintain and strengthen their negative Black self-concept if such assumptions go unchallenged or fail to get dispelled (Evans et. al, 2010). To highlight another important take away from Sector five's stage four model, is this idea of the Immersion-emersion stage. Evans et. al, (2010) summarize Cross' (1991) explanation of what happens here in the best terms: 
Entering immersion, individuals have a clear sense of the identity they wish to shed, but have little information about the identity they wish to assume. As a result, individuals go through an inbetween phase in which they connect themselves to symbols of black identity (hair style, clothing, music, language; Cross, 1991). In some cases, individuals may take on a "blacker than" attitude. However, as individuals move away from an antiwhite perspective toward a pro-black vision, they begin to focus on nurturing a connection and commitment to black people. In emersion, individuals begin their transition towards stage four, internalization, by reexamining, through a more balanced and focused lens, the coalescing of the affective and cognitive aspects of black identity. (p. 259)

This direct language provides a very rich description of what is theorized to happen to those who have immersed themselves into Black culture initially and later begin to pull back from the anti-white agenda to take on a more altruistic understanding of a Black Identity. To help with this idea of exploration and understanding of other racial groups, it would be important to note the literature of Helms $(1952,1992)$ on White Identity Development (WIDM) and Row, Bennett, and Atkinson's (1994) White Racial Consciousness Model (WRCM).

White Identity Development and White Racial Consciousness Models. Helms (1995, 1992) created a model for people of color and the White Identity Development model to increase White people's awareness of their role in creating and maintaining a racist society, whereby prompting the need for their action in dismantling it. There were two sequential phases: abandonment of racism and the evolution of $a$ nonracist identity. The latter of which calls for individuals to begin the quest to understand themselves as racial beings and owning the privileges given for being White, while redefining themselves and learning about other racial groups. This idea of abandonment of previously taught ideas and exploring others is similar to what was found in Cross and Fhagen-Smith's Black Identity Development model as well as both RCID and IDD mentioned earlier. Other literature from Row et. al (1994) informs our understanding of the 
White Racial Consciousness model and how it was seen to move beyond previous White racial identity models including WIDM. To be clear, their issue was that previous models suggested a parallel identity development process with people of color and how they neglected to focus on the actual development of White Identity, but rather having described the development of different attitudes toward sensitivity for other racial/ethnic groups (Evans et al., 2010). On the contrary, the WRCM involves "ones' awareness of being White and what that implies in relation to those who do not share White group membership" (Rowe et al., 1994, pp. 133-134). The assumption, according to Row et al. (1994) was that white racial consciousness and racial awareness were related; therefore, the way in which dissonance is resolved is the main cause for a change in racial attitudes. The model used "types" instead of stages to speak against the notion of a linear progression; the two types are unachieved White racial consciousness and achieved White racial consciousness. The theory here is that whether positive or negative, a significant amount of dissonance is what determines the type one moves into. To avoid delving into the details associated within each type, I bring to surface some important ideas that will provide the gist for understanding this theory. Evans et al. (2010) Shared that individuals with a dominative type attitude are ethnocentric and firmly believe in their superiority over people of color; they use negative stereotypes as a backdrop for their knowledge for people of color. Furthermore, those who passively possess this attitude may avoid interaction with people of color all together and perhaps, never admit this or realize that their beliefs and actions are contradictory. Moreover, those who oppose discriminatory practices, but are not in favor of programs or procedures designed to eliminate or reduce it possess a conflictive attitude. This attitude is the foundation for our previous mention of meritocracy and the idea that people of color have equal opportunity now and are responsible for their own issues. On the other hand, a reactive racial attitude recognizes the inequities and injustices that exist for people of color to bear the burden. Here, individuals know and feel for people of color, but are not active in their approach to challenge the dominance of a White status quo. In contrast, to this attitude is the integrative racial attitude, which is committed to social 
change and activism while maintaining genuine relationships with people of color. Although, much of this has been a dichotomy between Black and White identities, our life-worlds are reflective of other identities including Latino/a people, who have historically been oppressed and underserved. Now that the racial identity theories of Black and White identities have been reviewed, it is necessary to discuss how Latino Identity Development is framed in the literature.

Latino Identity Development. Ferdman and Gallegos (2001) asserted that previous racial constructs within the U.S. do not easily apply to Latinos; forcing them to fit shortens and distorts Latino realities. Of particular note, race is secondary to Latinos, skin color plays a significant role in how one is valued based upon skin tone (e.g. devaluing of those with darker skin) and it is difficult to place Latinos in a finite racial category because of their mixed heritages and the multiplicity of skin gradations. Also, some Latinos choose to identify as White, others reject being White, while some use Latino as their racial and ethnic group. Much of this might be determined by their physical appearance, familial influences etc. To frame these experiences into theory, Ferdman and Gallegos (2001) use orientations instead of stages used in aforementioned theories to make clear that this theory on Latino identity is neither cyclical nor linear; meaning that people may correlate with several or one orientation for their life-span. These orientations include: (1) Latino-integrated (willingness to challenge racism and views self within larger multicultural framework), (2) Latino-identified (Pan-Latino identity; Recognize entire Latino community as encompassing one Latino race), (3) Subgroup-identified (Identify with a specific Latino subgroup based on inferior/superior beliefs and is also aware of White oppression on their subgroup), (4) Latino as other (Do not know their specific background within the heritage, but may be inclined to interact with other Latinos because of physical characteristics), (5) Undifferentiated/denial (the adoption of a color-blind philosophy and living according to dominant culture because racism is only experienced individually and not via systemic racism), (6) White-identified (The adoption of a White identity, lives as White and sees Latino and other racial groups as inferior). Throughout all the previously mentioned racial identity theories, we come 
to understand the points at which an individual is essentially clueless/careless, interested/explorative, zealous and one-minded, open and willing to learn, in denial or unbothered as it pertains to their racial or ethnic background and those of others. Thus, it is important to have an awareness of how people, we interact with daily or within our life-worlds, mediate race and all its diversity and social justice facets. Racial identity theories are useful in their ability to frame our understanding of the people within our human ecological systems, to create outcomes more tied to notions of equity and inclusion. Therefore, it is important to make note of the ways practitioners have promoted diversity, social justice and racial identity development outcomes through curricular and co-curricular interventions. Such concern and participation with the identity development process for students and faculty-administrators has the potential to shift fixed mindsets and learned behaviors, while swinging the pendulum of social constructs inside the ecological system in ways they can be properly understood and mitigated for campus reform.

\section{Curriculum and Student Organizations: Understanding more on Campus Reform}

\section{Reform through Student Organizations}

In like manner, Harper and Quaye (2007) studied the phenomenon of "Student Organizations as Venues for Black Identity Expression and Development among African American Male Student Leaders," which tie into this idea of a co-curricular intervention. In their review of the literature, Taylor and HowardHamilton (1995) were found to have examined the relationship between student engagement and African American male student's racial identity attitudes. In fact, they found that higher levels of out-of-class engagement contributed to strong racial identity attitudes in a study of $n=117$ participants across 10 predominantly white institutions (PWIs). Of note, the highly involved students were at Cross's (1995) Immersion-Emersion and Internalization stages, while the less engaged participants reported preencounter attitudes at high levels. Harper and Quaye (2007) explored this idea further in their qualitative study of $n=32$ African American undergraduate men across six midwestern universities. Each of the 
participants reported a commitment to uplifting the African American community by attempting to dispel stereotypes, breaking down barriers, and opening doors as a result of their co-curricular involvement in primarily Black student organizations. Likewise, some reported having joined predominantly White student organizations to help fund minority initiatives, Black speakers, musicians, and entertainers and to increase opportunities for collaboration between these groups and Black organizations. To validate this claim, one student participant reported that "I joined Union Board because they bring all the concerts to campus. I noticed that they kept brining nothing but White artist. Because of me, Union Board and Black Student Union co-sponsored the first hip-hop concert we've ever had" (Harper and Quaye, 2007, p. 136). Through this example, we can see student organizations as interventions in achieving diversity outcomes as a result of campus programming that shifted towards more diverse entertainers. Additionally, it was also reported that, for those who worked within diverse student organizations, students came to appreciate the differences of others and were able to teach people about their backgrounds, life histories and Black culture (Harper and Quaye, 2007). Overall, Harper and Quaye (2007) argue that it was leadership and engagement in student organizations, which allowed participants to experience Cross's (1995) internalization stage of having inner comfort with forming alliances with those outside ones' own racial group for the purpose of social justice for African Americans and other oppressed groups. Although, Harper and Quaye (2007) found positive outcomes for diversity and social justice with co-curricular interventions, Krings, Austic, Gutiérrez, Dirksen (2015) explore this phenomenon by looking at "The Comparative Impacts of Social Justice Educational Methods on Political Participation, Civic Engagement, and Multicultural Activism."

\section{Service Learning, Intergroup Dialogue or Lecture-based Diversity Curricular Reform Approach}

In a cross-sectional, repeated measures, quasi-experimental study using $n=653$ college students at a midwestern university, they sought to understand the commitment and confidence in these areas as a result of their participation in either service learning, intergroup dialogue or a lecture-based diversity class, 
while using a general psychology course as a non-intervention group in the study. Of importance, social justice education course content usually is structured in such a way that it helps students understand racial dynamics, and their role in critical analysis, social change and personal reflection. These courses aid them in answering the call to for schools to produce students who are committed, engaged and socially responsible (Krings et al., 2015). These outcomes are consistent with the service-learning concept (intervention) but through the collaboration of community-based learning, which attempts to respond to the needs of marginalized groups. After examining the outcomes of the participants from each type of intervention, Krings et al., (2015) found that those who participated in the service-learning course ended with the highest-level participation in political participation, civic engagement and multicultural activism. Those with the intergroup dialogue intervention showed increases in all three outcomes. Likewise, the lecture based social justice course showed increases in political and multicultural activism, but not quite civic engagement.

\section{Dedicated Diversity Course, Infusion Model or Egalitarian Approaches in the Curriculum}

Similarly, Pitner, Preister, Lackey, and Duvall (2018) also explored interventions to determine which yielded more effective results in their article "A Dedicated Diversity Course or an Infusion Model? Exploring which Strategy is More Effective in Social Work Pedagogy." In their quasi-experimental study of n=286 Master of Social Work (MSW) students, they discovered that a dedicated diversity and social justice course as most effective at increasing students' cultural responsiveness and social justice awareness. To ensure clarity, the study compared the outcomes of students who completed a social work course dedicated to diversity and social justice with those of students who completed a course with the infusion intervention, whereby diversity and social justice was "infused" into a course, but not the sole purpose of the course. Along similar lines, Bennett and Sekaquaptewa (2014) tested an intervention strategy within an engineering school among men. Given the underrepresentation and perhaps, sexist treatment of women in the engineering field, the researchers sought to determine the ways in which attitudes and 
behaviors of men might lean more toward affirming the presence and abilities of women, while viewing them as counterparts rather than inferior or not belonging within engineering programs. In a quantitative study of 129 students enrolled in an introductory engineering course, one group was given the egalitarian social norms message-which explicitly spoke against racial and gender bias-while others in the control group did not receive this message. As a result, by the end of the courses, the treatment (intervention) group were found to have stronger intentions to speak out against racist behaviors and held more positive attitudes towards diversity. Thus, Bennett and Sekaquaptewa (2014) produced results that suggested that the egalitarianism and intolerance of bias tone set for incoming students have the potential to create a more inclusive environment; especially in a field dominated by White males. Consistent among the research thus far, is the success of explicit and dedicated interventions that highlight and target diversity and social justice.

Overall, through the language of IDD, a social identity theory, we can better understand the interworking of racial identity theories, of which provide a framework for our knowledge of various raced groups. It is through these theories that we determine our cognitive, affective and behavioral responses to for both ourselves and those of whom we interact with. While everyone may not be exposed to these theories to know where they, themselves, fall on the continuum, it is likely that much of what is theorized will be experienced. But knowing and being aware can increase the chances of individuals becoming more inclined towards activism and collaborating with others to achieve outcomes that favor equity and disdain for oppressive structures. To this end, what I have found in the literature regarding curricular and cocurricular interventions, gives us the tools to achieve positive outcomes related to diversity, social justice and racial identities. Intergroup dialogues, service learning, dedicated diversity courses (lecture or intergroup structured), and an egalitarian social norms approach in traditionally biased spaces, all serve as interventions, of which institutions of higher learning can use to influence campus climate and more fully prepare its constituents for an ever-diverse society. Although, this literature points to the utility and 
favorable outcomes of the interventions, we stand to strengthen our knowledge base on the cognitive and psychological impediments to a people fraught with oppression as a result of our education system.

Furthermore, we must consider the large girth of information on organizational change, reframing organizations, and the rise of chief diversity officers as we come to understand how colleges change and what scholars recommend for those seeking to enact change within higher education.

\section{Understanding the Foundation of a Seminal Critical Pedagogy}

Drawing from the limited but wealth of radical literature of the past, this dissertation brings forward the seminal works of the British Philosopher, Paulo Freire, with his book Pedagogy of the Oppressed (POTO) originally published in 1970 and again in 1993 and 2000. Pedagogy of the Oppressed was considered so revolutionary among totalitarian states in Cape Verde, people risked cruel punishment or imprisonment for being caught reading it, as they along with other nations struggled to overthrow totalitarianism and oppression (Macedo, 2000). Freire's work ventured to people a philosophical understanding of the relationship between the oppressed and the oppressor. It exposed the various educational instances, structures and processes where oppression colonization was experienced by people labeled as underclass, poor, and less human. POTO dealt with liberation and education as mutual processes, the "Banking" concept of education as an oppressive instrument, problem-posing education as an instrument for liberation, dialogics and dialogue and several other important frames of thought. Given the expansiveness of POTO, this dissertation will not attempt to address all of Freire's philosophies presented throughout the text in detail, but those most germane to the direction of this study. Of note, in Macedo's (2000) introduction of Freire's (1970/2000) POTO, he initiates our understanding of the use of the word "pedagogy." He states that very few liberal scholars who embrace Freire's work view pedagogy as more than just a teaching method in effort to see it as a philosophy or a social theory. In fact, "pedagogy" according to its Greek roots translates as "to lead a child;" Macedo (2000) concluded that the term illustrates that education is fundamentally directive but must always be transformative. He goes on 
to reference Stanley Aronwitz, who argued that “Freire's pedagogy is grounded in a fully developed philosophical anthropology, that is, a theory of human nature, one might say a secular liberation theology, containing its own categories that are irreducible to virtually any other philosophy (Macedo, 2000, p. 25). While Freire sought to, as Frantz Fannon termed, transforming "the wretched of the earth from being for others to beings for themselves," he also wanted to acknowledge his indebtedness to the philosophical traditions of Marx, Gramsci, Hegel, and Sarte among others (Macedo, 2000). As the focus in this study addresses notions of oppression by studying campus racial climate and the demands for a culturally inclusive curriculum, it is foundationally necessary to extrapolate from the literature traditional views on oppression, education and how people learn to be liberated in educational spaces-in this study's case, higher education. To underscore a piece of Freire's philosophy on education and fully become aware of his desired approach to attaining true knowledge, he frames it best as he stated:

In order to understand the meaning of dialogical practice, we have to put aside the simplistic understanding of dialogue as a mere technique. Dialogue does not represent somewhat false path that I attempt to elaborate on and realize in the sense of involving the ingenuity of the other. On the contrary, dialogue characterizes and epistemological relationship. Thus, in this sense, dialogue is a way of knowing and should never be viewed as a mere tactic to involve students in a particular task. We have to make this point very clear. I engage in dialogue not necessarily because I like the other person. I engage in dialogue because I recognize the social and not merely the individualistic character of the process of knowing. In this sense, dialogue presents itself as an indispensable component of the process of both learning and knowing (P.17).

The words of Freire above, seem foundational to current curricular traditions of intergroup relations dialogue referenced earlier in this paper. It validates the importance and necessity for a practice that seeks to elicit knowledge and learning rather than linking what surfaces from dialogue to various inquiries into 
political, cultural and social contexts etc., which have the power to produce ideas and opportunities for liberation. Macedo (2000) goes on to note that some so-called Freirean educators fail to make the goal of dialogical teaching a process of knowing and learning that perpetually encompasses theorizing about the experiences shared in the dialogue process, by using it as a method. In the same vein, Macedo (2000) helps us to understand that Freire never wanted educators to engage in a pedagogy that would overdose on the experiential celebration which leads to a reductionistic view of identity. In doing so, Macedo (2000) shares Henry Giroux's argument that such pedagogy would cause identity and experience to be unconcerned with the problematics of power, agency and history; an overindulgence in the voices and experiences caused educators to fail at moving beyond difference that is structured binarism and uncritical appeals to the discourse of experience. Macedo (2000) argued that said approach transforms Freire's notion into a method invoking conversation that gives participants a group-therapy session for sharing grievances; a space that bell hooks described as nauseating because it brooks no dissent. To be clear, Freire was not against or trying to eliminate the idea of learning from the shared lived experiences of others, but rather was concerned at the connection those experiences has to politics, culture, policy etc., in order to question the interplay and root causes of oppression, to learn from and create the kind of knowledge to help free oppressed people to fight for change. In many ways, parallels can be drawn from this philosophy to previously addressed literature previously presented in this chapter, which highlights the widespread demands by Black students to require some form of a course/ curriculum infused with an opportunity to engage in critical dialogue about race, racism, privilege, oppression and social justice. As stated earlier, it is through cultural exploration and opportunities for intergroup relations dialogue to occur, that people begin to discover new knowledge, their identity and that of those around them and ignite the flame to tear down oppressive structures. In other words, if people are never taught or are afforded this opportunity on their educational journey, they may never realize their cause of or place in oppression, to know what organizational change is necessary; they may also struggle to recognize false generosity from those is 
positions of power. To underscore these notions, Freire (1970/1993) cautions us about the mindsets and behaviors of oppressed people seeking to rise from dehumanization to become more fully human. He urges the oppressed not become oppressors of the oppressors, but restorers of humanity for both, for the struggle to have meaning. Freire $(1970 / 1993)$ proclaims that the greatest humanistic and historical responsibility of the oppressed is to liberate themselves and their oppressors; only the power that comes from weakness will have the ability to free both. He goes on to say that attempts to "soften" the power of the oppressor in defense of the oppressed almost always reveals false generosity. Earlier in this chapter under General Overview of Campus Racial Climate \& the Journey to Creating Inclusive Climates, I recall attention to the experiences of Black students -in Reddick and Jones (2017) study-who lamented about their administrators throwing money at them. Money to support various programming but failed to root their support for students as ongoing institutional practices with policies to legitimize and ensure its stability. I believe this is the crux of what Freire (1970/1993) sought to warn us about; in contrast, he believed true generosity consists of fighting to destroy the causes, which nourish false charity. Central to this study, is an in depth look into Black student demands and how these demands might become interventions at Predominantly White Institutions. Freire's (1970/1993) work provides us with a backdrop-relatable enough for campus reform efforts-through his philosophy on how the oppressed people should approach change and structural racism. He proclaims:

This lesson and this apprentice must come, however, from the oppressed themselves and from those who are truly solidary with them. As individuals or as peoples, by fighting for the restoration of their humanity they will be attempting the restoration of true generosity. Who are better prepared than the oppressed to understand the terrible significance of an oppressive society? Who suffer the effects of oppression more than the oppressed? Who can better understand the necessity of liberation? They will not gain this liberation by chance but through the praxis of their quest for it, through their recognition 
of the necessity to fight for it. And this fight, because of the purpose given it by the oppressed, will actually constitute an act of love opposing the love-lessness which lies at the heart of the oppressors' violence, love-lessness even when clothed in false generosity (p. 45).

This quote drives home the basis for why students make demands on their campuses today; it justifies and reminds students and higher education professionals of the collective work and responsibility, necessary to bring about change rooted in love and good intentions on their campuses. However, there are those students who do not see the need to seek liberation or possess the drive to fight for a more humanizing place in society. Freire (1970/1993) blames this on the premise that during an existential experience of the oppressed, they adopt an attitude of "adhesion" to the oppressor; while they know they are downtrodden, this mindset does not allow them to objectivize or see the oppressor outside of themselves. Hence, some people's perception as being oppressed is impaired by their submersion in the reality of oppression. Likewise, they do not signify their binary nature to the oppressor as a reason to engage in the struggle to overcome, but rather their model of "manhood." (Freire, 1970/1993) Freire (1970/1993) contends that those who begin to recognize themselves as oppressed, must become developers of "the pedagogy of the oppressed," which he describes is the "pedagogy of the people" engaged in a fight for their own liberation. With it being cloaked in false generosity and objectifying humankind, Freire (1970/1993) proclaimed the pedagogy of the oppressed an instrument of dehumanization, which he contends makes it contradictory for the oppressed to be the developers of a liberating education given their positionality. This is why Freire believed that both men and women (people) must first deal with their consciousness as oppressors and oppressed people by considering their behavior, ethics and view of the world. It must be understood that oppressed people cannot be the initiators of oppression when they themselves are the recipients of violence and subjugation. Freire (1970/1993) says to determine this, "Any situation in which "A" objectively exploits " $\mathrm{B}$ " or hinders his and her pursuit of self-affirmation as a responsible person is one of 
oppression" (P. 55). Dialectically speaking, what this describes is a need for reflection, which Freire (1970/1993) deemed as essential to transformative action, which does not transform itself; in doing so, he urged for dialoguing with people (the masses) about their actions rather than explaining to people. Thus, invoking the need for the critical intervention of the people through the praxis of dialogue. Similarly, Freire's work in the 1970s is essentially rearticulated as we have come to understand the notion of intergroup dialogue (IDG). Previously, in this chapter, literature describing and advocating for the use of intergroup dialogue as a pedagogical praxis in educational settings to aid in the resolution of conflict among social groups was referenced. As a reminder, IGD's are face-to-face sessions that promote evocative engagement between members of two or more social identity groups where potential conflict exists or who have a history of conflict (Zúñiga et, al, 2002, p. 232-248). I recognize the forward-thinking and recurrence in the literature among Freire, Zúñiga et, al. and their advocacy and impact on the praxis of true dialogue; one that should be used as a Critical intervention in manners of social conflict and forms of oppression. Albeit, higher education practitioners and scholars must consider the true intent, caveats and warnings Freire presents to ensure proper praxis of dialogue. Freire (1970/1993) challenges us to view the essence of the word "dialogue" and its purpose as more than just an instrument that makes dialogue possible, but one that contains two dimensions: reflection and action. If one word (dimension) is sacrificed, it is to the detriment of the other as the word, when used in its truest sense, is a praxis; one of which can transform the world when properly utilized. He argues that when a dichotomy of a word imposes upon its constitutive elements, it renders the word as unauthentic; unable to transform reality (Freire, 1970/1993). To underscore this perspective, Freire (1970/1993) declared "when a word is deprived of its dimension of action, reflection automatically suffers as well; and the word is changed into idle chatter, into verbalism, into an alienated and alienating "blah." It becomes an empty word, one which cannot denounce the world, for denunciation is impossible without a commitment to transform, and there is no transformation without action. (p. 87) Likewise, if only action is emphasized in the absence of reflection, it becomes activism which 
yields the notion of action for action's sake; thereby refuting the true praxis to make true dialogue possible. The kind of dialogue Freire believed needed to be nourished by true words of men and women (people) who should be able to discover its problems, transform, name, and change the world; to that, he argued was to exist humanly. However, he believed that because this dialogue was to be mediated by the world to name the world, dialogue could not occur with those who do not seek to name the world (transform it), as they would constantly deny others the right to speak of their experience, to ultimately prevent the persistence of dehumanization and subjugation. In other words, all parties must act humanly to bring about change. This is important for practitioners and students who are involved in campus reform efforts; creating and establishing a safe space to engage in Critical dialogue is important for producing an improved campus racial climate and institutional change outcomes. As we have come to understand more about the

pedagogy of the oppressed, juxtaposed to the vast literature on campus racial climate, we are faced with a multitude of historical and pervasive problems related to the discontinuities in race relations; one that requires this study to delve deeper into what knowledge exist to reframe these organizations and the various organizational change theories and practices to consider for those interested in campus reform.

\section{Organization Change}

Higher education institutions are by far not the only places where racial climate is tense, nor are they the only organizations that fester internal dilemmas unrelated to race, but that cause an impediment to healthy work environments and successful outcomes. Thus, there is much knowledge to be gained from theories and models developed for the sole purpose of addressing organizational change or reframing from a general perspective; of which may be used in both educational and non-educational settings. Because I am studying issues with the academic and non-academic experiences of people, primarily Black 
students, as mediated by the discontinuities in race, I am inevitably led to infer that some of these issues must stem from flawed, stagnant, oppressive, or outdated organizational systems and structures. Hence, I was inspired to consult the literature to understand more about these models and theories, which may serve as a resource for institutional change agents. Bolman and Deal (2008) produced a four-frame model in their text Reframing Organizations which include the: Structural, Human Resource, Political, and Symbolic frames. Each frame is presented with its own outline that describes its root functions in four categories: metaphor for organization, central concepts, image of leadership, and basic leadership challenges. Through these categories, Bolman and Deal provide us with an overview of each frame to understand the importance of multi-frame thinking when attempting to develop a strategy for reframing an organization with problems, Table 1 is a visual depiction of these four frames in summation.

\section{Table 1}

Overview of the Four-Frame Model

\begin{tabular}{|c|c|c|c|c|}
\hline & Structural & Human Resource & Political & Symbolic \\
\hline $\begin{array}{l}\text { Metaphor for } \\
\text { organization }\end{array}$ & $\begin{array}{l}\text { Factory or } \\
\text { machine }\end{array}$ & Family & Jungle & $\begin{array}{c}\text { Carnival, temple, } \\
\text { theater }\end{array}$ \\
\hline Central concepts & $\begin{array}{c}\text { Rules, roles, goals, } \\
\text { policies, } \\
\text { technology, } \\
\text { environment }\end{array}$ & $\begin{array}{l}\text { Needs, skills, } \\
\text { relationships }\end{array}$ & $\begin{array}{l}\text { Power, conflict, } \\
\text { competition, } \\
\text { organizational } \\
\text { politics }\end{array}$ & $\begin{array}{c}\text { Culture, meaning, } \\
\text { metaphor, ritual, } \\
\text { ceremony, stories, } \\
\text { heroes }\end{array}$ \\
\hline $\begin{array}{l}\text { Image of } \\
\text { Leadership }\end{array}$ & $\begin{array}{c}\text { Social } \\
\text { architecture }\end{array}$ & Empowerment & Advocacy & Inspiration \\
\hline Leaders is & Analyst, Architect & Catalyst, Servant & $\begin{array}{l}\text { Advocate, } \\
\text { Negotiator }\end{array}$ & Prophet, Poet \\
\hline $\begin{array}{c}\text { Basic Leadership } \\
\text { challenge }\end{array}$ & $\begin{array}{l}\text { Attune structure } \\
\text { to task, } \\
\text { technology, } \\
\text { environment }\end{array}$ & $\begin{array}{l}\text { Align organization } \\
\& \text { human needs }\end{array}$ & $\begin{array}{l}\text { Develop agenda } \\
\text { and power base }\end{array}$ & $\begin{array}{c}\text { Create faith, } \\
\text { beauty \& meaning }\end{array}$ \\
\hline
\end{tabular}

Note. Listed as Exhibit 1. in Bolman and Deal, 2008, P. 18 
As I continue to extrapolate meaning from this literature, I will expound upon some of the categories depicted in the model. Additionally, Bolman and Deal (2008) emphasize their focus on, as well as the importance of, both, management and leadership; arguing that some modern organizations require brilliant sparks of vision by wise leaders and the objective perspective of managers. At the heart of what they do, Bolman and Deal (2008) make an appeal to managers and leaders by avowing:

We need versatile and flexible leaders who are artists as well as analysts, who can reframe experience to discover new issues and possibilities. We need managers who love their work, their organizations, and the people whose lives they affect. We need leaders and managers who appreciate management as a moral and ethical undertaking. We need leaders who combine hard-headed realism with passionate commitment to larger values and purposes. We hope to encourage and nurture such qualities and possibilities. (p. viii)

Immediately, this quote presents parallels to the work of Paulo Freire and his insistence on humanity, being more human-like and concerned with the inhumane or inferior treatment and structures that some people experience. To do this, Freire (1970/1993) argued against false generosity toward oppressed individuals, but rather for the willingness to hear their experiences and seek for their liberation. Doing so, validates Bolman and Deal's (2008) philosophy that managers and leaders must reflect upon their moral and ethical stance and possess a love for the people (care for humankind). In like manner, Bolman and Deal (2008) provide some example content, which has been placed in Table 2, on expanding managerial thinking to consider by using an engineering and art analogy to describe the various manager styles. 
Table 1

Expanding Managerial Thinking

EXPANDING MANAGERIAL THINKING

\begin{tabular}{|c|c|}
\hline \multicolumn{2}{|c|}{ EXPANDING MANAGERIAL THINKING } \\
\hline How Managers Think & How Managers MIGHT Think \\
\hline $\begin{array}{l}\text { They often have a limited view of organizations } \\
\text { (for example, attributing almost all problems to } \\
\text { individuals' flaws and errors }\end{array}$ & $\begin{array}{l}\text { - They need a holistic framework that } \\
\text { encourages inquiry into a range of } \\
\text { significant issues: people, power, } \\
\text { structure, and symbols. }\end{array}$ \\
\hline How Managers Think & How Managers MIGHT Think \\
\hline $\begin{array}{l}\text { Regardless of a problem's source, managers often } \\
\text { choose rational and structural solutions: facts, } \\
\text { logic, restructuring. }\end{array}$ & $\begin{array}{l}\text { - They need a palette that offers an array } \\
\text { of options: bargaining as well as training, } \\
\text { celebration as well as reorganization. }\end{array}$ \\
\hline How Managers Think & How Managers MIGHT Think \\
\hline $\begin{array}{l}\text { Managers often value certainty, rationality, and } \\
\text { control while fearing ambiguity, paradox, and } \\
\text { "going with the flow." }\end{array}$ & $\begin{array}{l}\text { - They need to develop creativity, risk } \\
\text { taking, and playfulness in responses to } \\
\text { life's dilemmas and paradoxes, focusing } \\
\text { as much on finding the right question as } \\
\text { the right answer, on finding meaning and } \\
\text { faith amid clutter and confusion. }\end{array}$ \\
\hline How Managers Think & How Managers MIGHT Think \\
\hline $\begin{array}{l}\text { Leaders often rely on the "one right answer" and } \\
\text { the "one best way"; they are stunned at the } \\
\text { turmoil and resistance they generate. }\end{array}$ & $\begin{array}{l}\text { - Leaders need passionate, unwavering } \\
\text { commitment to principle, combined with } \\
\text { flexibility in understanding and } \\
\text { responding to events. }\end{array}$ \\
\hline
\end{tabular}

Note. Listed as Exhibit 1.2 in Bolman and Deal, 2008, p. 20.

From the examples shown in Table 2 above, one might deduce that both mind-sets (engineering or artful) have their own unique place in any organization and that the Art analogy, listed under how managers might think, could be an enhancement, rather than a replacement for managers and leaders to consider. With this in mind, practitioners (managers, leaders etc.) should sure up their ability to lead effectively by increasing their knowledge of multiple perspectives or frames for leadership, to avoid being clueless about 
their decisions (Bolman and Deal, 2008). Bolman and Deal (2008) compare the utility of these frames to that of a map to aid in navigation, tools for solving problems, filters for sorting essence from trivia; all while being grounded in managerial wisdom and social science. Furthermore, research from Dunford and Palmer (1995) indicated significant positive short and long-term effects for management courses teaching multiple frames; 98 percent of participants rated reframing as helpful or very helpful and approximately 90 percent believed it gave them a competitive advantage. Kezar's (2018) work also supports a multiperspectives approach, as I discovered through her advocation for a multi-theory approach to how colleges change. So, what do these four-frames of leadership include within each frame? In summation, the structural frame deals with the architecture of organization, how units and subunits are designed, the rules, roles, goals and policies. The human resource frame accentuates getting to know people, their strengths and idiosyncrasies, reason and emotion, fears and desires. The political frame conceptualizes organizations as competitive arenas with scarce resources, competing interests while struggling for power and advantage. The Symbolic frame is concerned with issues of meaning and faith; ritual, ceremony, story, play, and culture are central to the heart of an organization's life. (Bolman and Deal, 2008) The authors make clear their assertion that any organizational problem or desire for change can be viewed from each of the four lenses to determine optimal effectiveness. While it is necessary to present literature on reframing and organizational change to inform our knowledge of how Black student demands might be implemented to elicit change, this dissertation does not attempt to cover the vast intricacies for each frame, model or theory scholars have detailed. To do so, would shift the focus of this study too far from its centrality on campus racial climate, Black student demands as interventions and the student and facultyadministrator experiences in the face of adversity. Therefore, I have pin-pointed various aspects of the organizational change literature I find most relative to this study.

Of importance to the four-frames model, I discuss each of them in greater detail to provide more context for their utility within higher education institutions. Before applying the lenses to a frame, 
the authors urge us to beware of the peculiarities of organizations. Bolman and Deal (2008) infer that: organizations are complex, challenging and erratic environments with unpredictable people and systems; organizations are surprising, what is expected is not always the outcome and today's solutions might me the futures downfall; organizations are deceptive, mistakes and surprises get camouflaged and subordinates are fearful to speak up at critical times to avoid disaster; organizations are ambiguous, difficult to pin point or draw conclusions to find a starting place for change because the facts may yield varying interpretations (Pp. 31-32). According to Bolman and Deal (2008) the structural frame is how an organization is designed and whether or not it will be tightly structured or flexible. They contend ridged bureaucratic structures are buried in red tape that impede upon employee morale and skill-utilization and work completion; thus, giving managers too much control over the people. On the contrary, Bolman and Deal (2008) compared the flexible, flat and entrepreneurial structure of BMW with that of GM and Ford which they described as ridged and bloated bureaucracies too slow to respond to market trends or competitive threats. Of resemblance to education, Bolman and Deal (2008) believe that the pressures of globalization, new technology, competition, customer demands and workforce undercurrents are causing organizations to rethink the design of their structure; they suggest a focus on social architecture to allow people to do their best, which requires flexibility and encouraging people to speak out to embrace new ideas. Managers and leaders should use either vertical or lateral coordination or a combination of both depending upon the organization's issues. The former being more prone to roles of authority, rules and polices, and control systems, while the latter includes task forces, coordinating roles, matrix structures and networks. (Bolman and Deal, 2008) All, of which higher education practitioners should become more familiar with when designing the structure of institutions, as context, human demographics, demands, technology, goals etc. are factors to consider. In fact, Bolman and Deal (2008) detail a number of structural dilemmas, some of which include but are not limited to: underuse versus overload, lack of clarity versus lack of creativity, excessive autonomy versus excessive interdependence, too loose versus too tight, and 
irresponsible versus unresponsive among others of which to consult for their implications on organizations. Given our interest in curricular interventions, Bolman and Deal (2008) discuss the professoriate as an example of professional bureaucracy, which is indicative of a structural dilemma. Using Harvard University, its structure was described as flat and decentralized given the few managerial levels between faculty and the topmost senior leadership; thus, professional training and indoctrination are heavily replied upon to control knowledge production. Therefore, faculty professionals are shielded from formal interference, allowing them-as highly trained experts - to operate best in their scholarly domain. However, Bolman and Deal (2008) claim that such separation, especially for tenured faculty, from professional bureaucracies make it difficult coordinate pedagogical practices and content knowledge or to assess quality control. External changes for reform efforts are slow to take flight, because faculty professionals may have their own scholarly aspirations and view them as annoying distractions; on the other hand, the institution may be calling for reform efforts to take form at a rapid speed. In fact, Harvard's president, Larry Summer was challenged in this professional bureaucracy when he suggested that the celebrity African American studies professor, Cornel West, redirect his scholarly pursuits. This private conversation became front page news of the New York Times, Summer's copious public apologies failed to rectify his grasp for scholarly control and West left for Princeton. The authors insist that in a struggle between the strategic apex and the operating core (faculty professionals), the professionals are likely to win given their constricted bond with their field than any one institution. (Bolman and Deal, 2008, Pp. 82-83) It would serve higher education managers and leaders well to consider these structural dilemmas when trying to bring about campus reform efforts. Perhaps, a more humanistic and people centered approach reflective of the human resource frame might be more beneficial to consider.

Central to the human resource frame is what organizations and people do for each other. This frame is concerned with the assumptions that organizations exists to provide human needs, to provide people with careers, salaries and opportunities; in return, they need new ideas, energy, and talent to 
succeed from people (Bolman and Deal, 2008). On the off chance, such harmony does not occur and one or both are exploited, both tend to suffer. As referenced earlier, Paulo Freire (1970/1993) compared said exploitive behavior to that of oppression, where the person being exploited becomes oppressed. To avoid a tense working environment, Bolman and Deal (2008) presented three things-from Cable and DeRue (2002) - related to workers concerns about "fit," how their skillsets will be utilized and if a job will meet their needs enough to be retained. The three things are: how well an organization responds to individual desire for useful work; how well jobs enable employees to express their skills and sense of self; and how well work fulfills individual financial and life-style needs (p. 122). To expound upon this frame, Bolman and Deal (2008) present an extension of Maslow's Hierarchy of Needs introduced by Douglass McGregor (1960), known as Theory $X$ and Theory Y. The notion is that Theory X managers (organizational structures) tend to make assumptions about working people, which often become self-fulling prophecies. In other words, managers believe that workers are lazy, need to be tightly directed and controlled; thus, instituting threats and punishments to ensure execution of responsibilities without a lack of success. In implementing the former strategies described, it was believed that the people would actually begin to fulfill the assumptions of their Theory X managers. On the contrary, McGregor (1960) argued, just as ones' dietary deficiency deems one as sick and yields a lack of physiological needs, which alter behavior, the deprivation of higher-level needs (safety, status, independence, social and egoistic) makes a person sick and will also yield behavior consequences of passivity, hostility and refusal to accept responsibility. Nonetheless, this enters McGregor's (1960) Theory Y position, which essentializes management conditions where people can achieve organizational rewards and satisfaction in their work with organizational requirements that are aligned with employee self-interest. In "getting it right," Bolman and Deal (2008) drew our attention to a summation of Basic Human Resource Strategies in exhibit 7.1 (See appendix I), of which include: Build and implement an HR strategy, hire the right people, keep them, invest in them, and promote diversity. For example, hiring the right people allows organizations to select candidates who "fit the mold" reflective of 
their overall goals and vision of service offered; to select people with positive attitudes, good interpersonal skills, a sense of humor, and are open to diversity etc. In addition, investing in employees' continued learning through training and development opportunities limits the likelihood of undertrained workers who bring down the organization with shoddy quality, poor service and costly mistakes. Although companies, according to Bolman and Deal (2008) are reluctant to invest in developing human capital, the cost is immediate, but easy to measure and long-term. They also reference Waterman's (1994) return on investment study at Motorola, which found that the company gained twenty-nine dollars for every dollar invested in sales training. To see more return on investment, Bolman and Deal (2008) suggested giving power (Empower employees) to employees and promoting egalitarianism; allowing them the autonomy to redesign work, foster teams, and imbue work with meaning. The underlying premise is treating people well, which includes ideals such as equity, inclusion, and the unmitigated insistence on fighting against discriminatory and racist practicing and promoting diversity. Bolman and Deal (2008) framed for us, the increase in lawsuits since the early 1990s into the new century; for example, Denny's Restaurants, Shoney's, Coca-Cola, Texaco, and Wal-Mart were all hit with lawsuits, stock-falls or became the biggest face of corporate discrimination in history, that resulted in a loss of $\$ 54$ million, $\$ 134$ million, $\$ 192$ million, a half billion in stock value drop, and a 1.5 million person (women) class action suit that reached the supreme court, respectively. It is for these reasons that we continue to see an uptick in the placement of chief diversity officers (CDOs) in not only corporate settings, but higher education institutions. Given the relativity of Bolman and Deal's (2008) insistence on promoting diversity and our research focus that is wrapped in ideals of diversity and campus climate changes, I briefly pause our four-frame model exposition, to discuss the role of a chief diversity officer.

\section{The Role of Chief Diversity Officers for Institutional Reform}

In line with Bolman and Deal's (2008) recommendations to "hire the right people" and "promote diversity," what better way might higher education institutions do this than to hire a person to 
lead these efforts? While this might seem logical and full proof, some scholars have varying opinions about the effectiveness of chief diversity officers on organizational change and diversifying an institutions' environment. First example, Alex-Assensoh (2018) contends that there is more to hiring a CDO to avoid failure in his "Hiring a Diversity Officer Is Only the First Step. Here are the Next 7" article in The Chronicle of Higher Education. Of note, he stresses the criticalness of chancellors, presidents and provosts, who must collaborate with CDOs to build a campus infrastructure where diversity, equity and inclusion are a part of everyday campus life. The first four steps Alex-Assensoh (2018) suggests are: (1) go first (diversify ones' own staff from the top down of others are expected to do so); (2) manage expectations (determine a collaborative change agenda with benchmarks that is clear, accessible, contains CDO's responsibilities and a reasonable timeline with routine communications on progress); (3) develop meaningful measures of success (Align CDO performance measures with campus strategy designed to incentivize faculty, staff and students and assess their role in collaborating with others to promote modernizations for programming and policies while creating a more diverse culture and providing support to underrepresented people on campus); (4) engage faculty members (Given their virtual ownership of the curriculum for which the institution receives its rankings, they should be engaged to garner their support in sustainable change in diversity efforts) (p.2). In somewhat opposition to Bolman and Deal's (2008) push for investing in people through training and development, Alex-Assensoh (2018) suggests institutions (5) fix systems, not people, because when underrepresented people leave a campus they rarely point to a lack of training or workshops, but rather unchecked discrimination, harassment, and inequitable treatment. He recognizes admiration and worth behind programs on mentoring faculty etc. but indicate that it only focuses on one aspect of a dilemma. Of particular importance to enhance diversity, Alex-Assensoh (2018) emphasized the importance of "fixing" the system "that undermine success for underrepresented groups. For example, fix the performance-review processes that are blind to issues of departmental climate so that manager, department chairs, and deans are better able to develop respectful working environments for all" (p.4). 
Likewise, replacing antiquated tenure and promotions processes that reduce ground-breaking research and teaching and to dismantle personnel procedures reminiscent of sexual harassment, racial discrimination and ADA violations. These crippling systems must change and the CDO must unify the campus community to recognize the barriers and form partnerships to eliminate them (Alex-Assensoh, 2018, p.4). The final two steps are to (6) provide adequate support and (7) LACE up. For the former step, Alex-Assensoh (2018) declared: diversity offices need to be in locations that indicate their significance in daily campus life; CDOs need regular access to senior leadership for change efforts with sufficient support for staffing, programming and innovation; and that donors should be consulted with state-of-the-art diversity ideas to be a resource for endowed faculty positions, student spaces and lectureships. And finally, Alex-Assensoh's (2018) notion to LACE up is: to Love (Be kind in the midst of spitefulness and patient with people but not injustice); Authenticity (Admit missteps and where help is needed); Courage (Don not use the CDO as a shield in the face of adversity, call it out and defend what is right); and Empathy ( Feel alongside the people being served and understand their perspectives even in disagreement). AlexAssensoh (2018) proclaimed that hiring a CDO without the aforementioned steps to follow "it's little more than a doomed public-relations stunt" (p.5). Along similar lines, Hansen (2018) shares the results of a study by an economics professor and his colleagues from Baylor University in an article entitled "Do Chief Diversity Officers Help Diversify a University's Faculty? This Study Found No Evidence." When asked about the paper's mention of universities only hiring CDOs to say they have them as a sign of commitment to diversity but not make a commitment to much internal change, Professor James West stated his colleagues took the assumption that university administrators were acting benevolently in their plans to establish a CDO. However, critics of the paper during review how they would know; the answer was that they did not know for sure given they would have to show negative effects on diversity, but it would be hard to pare out if a campus was hiring a CDO due to a lack of campus diversity to begin with. However, the National Bureau of Economic Research working paper by Bradley, Garven, Law and West (2018) used publicly 
available race and ethnicity hiring data from 2001-2016 for faculty and administrators at U.S. universities with Carnegie R1, R2, or M1 status and student populations of 4,000 or more. After using 462 universities in the dataset and the year in which the first executive-level CDO was hired, they were unable to find significant statistical evidence to show that the hiring of an executive level diversity officer affected preexisting growth in diversity for tenure and non-tenure track hires, faculty hired with tenure, or for university administrator hires (Bradley et al., 2018).Hansen's (2018) interview with one of the researchers, James West, revealed that the results of the study might be attributed to cabinet level administratorincluding a CDO-usually had no direct effect over department hiring given academic autonomy. However, West did suppose that a CDO who sets a positive tone for how underrepresented-minority faculty members are viewed might affect attrition. Additionally, Bradley et al. (2018) studied the "supply" of new PhDs and reported that in 2016, 5,043 unrepresented persons earned a PhD out of 35,719 PhDs earned for U.S. citizens and permanent residents, which is $14.1 \%$ of the total. $48.5 \%$ of the overall populations with a $\mathrm{PhD}$ reported having secured jobs in academia, but there were only $11.6 \%$ of diverse tenure-track hires and $13.7 \%$ non-tenure track hires with a substantial dissimilarity in the proportion of underrepresented minorities with PhDs by field from a low of 7.9\% in Physical Sciences and Earth Sciences to a high of 23.1\% in Education. The difference by subfield is even greater for those in Latin American History or Area and Ethnic Cultural \& Gender Studies-40\% and $8.2 \%$ respectively-compared to $6.4 \%$ in Physics and Astronomy and $9.6 \%$ in Economics. Thus, the conclusion from these results was that there is a limit number of PhDs in some academic fields for universities wishing to increase faculty diversity to choose from. (Bradley et al., 2018) Overall, Bradley et al. (2018) makes it clear that their "inability to identify a significant effect should not be interpreted as an argument that an effect does not exist" (p.23), but rather a topic that deserves a considerable amount of additional research given the array of factors that may have contributed to their lack of evidence in finding a significant effect. In fact, the Hansen (2018) article with James West revealed that their work may contain a very small significant effect but numerically too 
small to be detected amid other factors in the dataset. Because my research, thus far, has presented literature pinpointing the increase in faculty/administrators as a dominant demand from Black students across more than 70 institutions, I find it necessary for higher education administrators and students to consider when attempting to strategize a plan for campus climate change and reform. As pressures increase and power, conflict and culture issues arise, students and faculty-administrators working together for campus reform might find utility in considering the assumptions made within not only the structural and human resource frames of reorganizing an organization but those of the political and symbolic frames as well.

\section{Organizational Change Continued}

Hence, Bolman and Deal's (2008) political frame supposes that individuals and groups within an organization/coalition have continuing differences in a world of scare resources. The political frame's perspective is summarized by five propositions:

1. Organizations are coalitions of assorted individual and interest groups.

2. Coalition members have enduring differences in values, beliefs, information, interest, and perception of reality.

3. Most important decisions involve allocating scare resources-who gets what.

4. Scarce resources and enduring differences put conflict at the center of day-to-day dynamics and make power the most important asset.

5. Goals and decisions emerge from bargaining and negotiation among competing stakeholders jockeying for their own interest. (Bolman and Deal, 2008, pp. 194-195)

Bolman and Deal (2008) recommend that parties who wish to see their interest come to fruition use politics and power productively, which is contrary to how most people view the concepts. They infer that the two concepts can be used in a way that allows people to leverage their power to do bargaining and 
negotiations with other contenders who are seeking their own set of goals, structure and policies.

Therefore, managers and leaders within higher education would benefit from a perspective that suggests ways to manage competing interests - which is inevitable-in any organization, especially when there is diversity among people, culture and values present. Based upon research from the 1970 s through the 1990s, Bolman and Deal (2008) recommended four key skills for managers as politicians to exercise: (a) agenda setting, (b) mapping the political terrain, (c) networking and forming coalitions and (d) bargaining and negotiating (Kanter, 1983; Kotter, 1988, 1982, 1985, 1988; Pfeffer, 1992; Smith, 1988,Pfeffer, 1992; Pichault, 1993; Bellow and Moulton, 1978; Fisher and Ury, 1981; Lax and Sebenius, 1986). To break down one example of agenda setting in the political sense is to devise a statement of interests and a scenario for getting the goods; whereas, a structural framed agenda outlines the goal with a schedule of activities. Scholars, over the years have concluded two major elements for leaders with an "agenda for change:" (a) to create a vision that balances long-term interest of key parties and recognizing competing internal and external forces to create a strategy for achieving the vision (Kotter, 1988). To expound upon this idea, Pfeffer (1992) advocated for leaders to know what others care about and how they think; this puts a positive spin on the idea that politicians are arm twisters, if they twist the right arm based on the cares of the people. Kanter (1983) adds to this idea when he said, "While gathering information, entrepreneurs can also be 'planting seeds' - leaving the kernel of an idea behind and letting it germinate and blossom so that it begins to float around the system from many sources other than the innovator" (p.218). in other words, gathering information to develop a vision should not be done by the innovator seeking change alone but rather should include collective voices. Another skill of importance to reference-especially when major conflict has arisen or is likely to arise-is Networking and Building Coalitions because it calls for leaders to:

1. Identify relevant relationships. (Figure out which players you need to influence.)

2. Assess who might resist, why and how strongly. (Determine where the leadership challenges will be.) 
3. Develop, wherever possible, links with potential opponents to facilitate communication, education, or negotiation. (Hold your enemies close.)

4. If step three fails carefully select and implement either more subtle or more forceful methods. (Save your big guns until you really need them but have a Plan B in case Plan A falls short.) (Kotter, 1985; Bolman and Deal, 2008, p. 219).

After leaders navigate the terrain of the political assumptions and recommendations Bolman and Deal and other scholars have presented, shifting to a symbolic lens might allow leaders, managers and those in the "lowerarchy" to frame things based upon the mold of what Bolman and Deal (2008) developed to be the symbolic perspective of reframing organizations.

Bolman and Deal's (2008) symbolic frame is an umbrella that brings together ideas from an array of disciplines such as sociology, anthropology, organizational theory, magic, political science, and neurolinguistic programming with suppositions for leaders interested in organizational reform; they are:

1. What is most important is not what happens but what it means.

2. Activity and meaning are loosely coupled; events and actions have multiple interpretations as people experience life differently.

3. Facing uncertainty and ambiguity, people create symbols to resolve confusion, find direction, and anchor hope and faith.

4. Events and processes are often more important for what is expressed than for what is produced. Their emblematic form weaves a tapestry of secular myths, heroes and heroines, riuals, ceremonies, and stories to help people find purpose and passion.

5. Culture forms the superglue that bonds an organization, unites people, and helps an enterprise accomplish desired ends. (p. 253) 
How humans make sense of chaos in an ambiguous world is the focus of the symbolic frame and its central concerns are meaning, belief, and faith. Take for example, the American flag; it is a symbolic and powerful symbol for both those who hold it in high regard and those who view its symbolism as oppressive and imperialistic. The way we experience life, symbols and meanings derive from our cultural beliefs, which are often invisible to us because we accept them as the way things are or ought to be; neglecting the experience and stories of others (Bolman and Deal, 2008, p. 248). To address a few of the organizational symbols listed in the assumptions above, Bolman and Deal (2008) contend that myths are "the story behind" (Campbell,1988, p.254) to enlighten, share, legitimize and maintain harmony while transforming the workplace into a well-regarded institution and an all-inclusive way of life, while undergirding the values. The values are essentially an organizations' identity from senior leadership to the those invested in the day-to-day work and provide a sense of self-worth and meaning to what they do. If it is providing excellent customer service, it is evident and present regardless of whether it is included in mission statements and formal documents, because it is what people know and feel the organization to be. Similarly, the vision takes the organizations' sense of purpose and core creed and frames it as an image for the future. It becomes a shared magical dream that brightens the path toward new possibilities with its myths and values at the center (Bolman and Deal, 2008). Other organizational symbols to address are stories and fairy tales, which give voice to inner conflicts and tensions and can give direction, comfort, encouragement and hope for people of any age. They continue to spread values and feats of heroines that have impacted an organization or similar spaces to underscore purpose and hope for the future. Bolman and Deal (2008) reflected on a story told by former Chancellor of Vanderbilt University, Joe B. Wyatt, at their annual convocation with several hundred professors and staff. The chancellor told a story about a second-grade teacher who consistently noticed classroom materials were missing and being taken by a student. The teacher met with the child's mother to inform her of the thefts over time to rectify; the mother explained to the teacher that the child comes home every afternoon and plays school and 
pretends she is you (the teacher). This story was a way for the chancellor to place emphasis on the universities core values of teaching in a dramatic way. Stories are a way to share human experience and give voice to those otherwise not heard or understood; they can be used in eight categories listed by Bolman and Deal (2008) ascribed by Denning (2005) for:

\section{Sparking action}

2. Communicating who you are

3. Communicating who the company is-branding

4. Transmitting values

5. Fostering collaboration

6. Taming the grapevine

7. Sharing knowledge

8. Leading people into the future (p.260)

For example, J.W. Marriott Sr., founder of Marriott hotels died a while ago, but stories to depict his steadfast commitment to customer service and his philosophy of taking good care of employees so they will be more inclined to take good care of the customers are still uttered today to continue this way of practice (Bolman and Deal, 2008). And finally, on symbols, Bolman and Deal (2008) proclaimed that metaphors, humor, and play have the "as if" factor of symbols; they view things as though they might be, out to be or could be. Metaphors have the ability to repurpose complicated issues of an organization into understandable images to influence attitudes, behaviors and places into perspective whether a university head leads the institution like a factory, craft guild, shopping center or beloved alma mater (Bolman and Deal, 2008). For example, a manager may see their organization as: a maze but want it to become a welloiled machine; aggregation of tribes with competing agendas but want it to become a symphony orchestra; herd of cattle on the rampage but want it to become a fleet of ships heading for the same port (Bolman and Deal, 2008, P. 268). Humor shows flexibility and adaptiveness and can save face in light of 
errors and express skepticism rather than spaces that remain too serious and tense. Play can open the door for relaxed rules, alternative exploration, experimentation, and creativity to elicit innovation with any activity or project. Each of these symbolic forms, when placed into a higher education context, would serve higher education practitioners well to consider when seeking to reframe and reform. But leaders would do well to consider the type of change or reforms needed and how to deal with or implement change efforts as theorized by organizational change theory and development research and an understanding of how colleges change.

There is a litany of literature from organizational change/development theoreticians that principally focus on several models for successfully implementing change and the type of change to employ, contingent upon the circumstances of the organization/institution needing change. Given the vastness of the intricate details on this body of research, I will only address in this dissertation, a summary of a few models and extant knowledge most relevant to the study.

\section{Episodic and Continuous Change}

Change, for an organization, deals with the notion of difference and how organizations function, the form they take, who the leaders and members are, and how they allocate resources (Huber, Suctcliff, Miller, and Glick, 1993). In terms of organizational development, Porra \& Robertson (1992) declare change to be "a set of behavioral science-based theories, values, strategies, and techniques aimed at the planned change of the organizational members on-the-job behaviors" (p.723). Lewin (1951) developed three stages of change: unfreeze, change, and refreeze which have been a universal formula for organizational development and undergirds his assertion that one cannot understand a system until their attempt to change it. Additionally, Random House's (1987) definition for the tempo of change provides us more perspective on change, as we understand this potential barrier to be the "characteristic rate, rhythm, or pattern of wok or activity." Weick and Quinn (1999) conducted an analysis of organizational change at 
the turn of the century to delve into the tempo of change by contrasting episodic change and continuous change against five properties Dunphy (1996) proposed surface in any comprehensive theory of change. They are: (a) a basic metaphor of the nature of organization; (b) an analytical framework to understand the organization that specifies both a direction for change and values to be used in assessing the success of the change intervention (e.g. survival, growth, integrity); (d) an intervention theory that specifies when, where, and how to move the organization closer to the ideal; and (e) a definition of the role of change agent (p. 543). When organizations experience a widening misalignment between an inactive deep structure and apparent environmental demands, it constitutes divergence, because the organizations have strayed from their steadiness. Such conditions create episodic change, which group together organizational changes that are intermittent and planned. (Weick and Quinn, 1999, p. 365) On the other hand, Continuous change refers to organizational changes that are constant, evolving and collective; in this case, when there are no a priori intentions, new patterns of organizing emerges (Orlikowski, 1996). Weick and Quinn (1999) contend that "the distinctive quality of continuous change is the idea that small continuous adjustments, created simultaneously across units, can cumulate and create substantial change" (p. 375). In other words, taking time to reflect, assess, learn and experiment with old and new practices while carefully and continually making enhancements to things, will make strides toward reform efforts that collectively become noticeable change. On the contrary, a basic metaphor of episodic change is imitation, which implies that for every theory of organizational change, leaders consider adopting what they see other organizations doing by observing one another for advancements in growth strategies and organizational structure (Sevon, 1996; Weick and Quinn, 1999). This type of imitation is perceived with the previous mention of institutions hiring chief diversity officers; some, to actually bring about cultural changes in diversity and others who do so to compete with a growing trend in organizational leadership structure. Additionally, Weick and Quinn (1999) assert that punctuated equilibrium, the edge of chaos and second-order change are also basic metaphors to help understand episodic change. When organizations 
are steady and not progressive, effectiveness decreases and people begin to increase pressure for change and revolutionize; usually resulting in an episode of basic change in action patterns and personnel for a new period of equilibrium (Tushman \& Romanelli, 1985; Weick and Quinn, 1999). The edge of chaos refers to the idea that organizations have several simple elements linked together by nonlinear and complex relationships; the system has both positive and negative responses and is back and forth between stable or instable. The last metaphoric example is second-order change, which makes the organization a place where shared beliefs work together for coordinated action to either bend or replace first-order change (Same philosophy or structure with minor changes) with deep change (Second-order change; different philosophy or structure with major changes) (Weick and Quinn, 1999). These ideas resemble Bolman and Deal's (2008) four frame model as its centrality deals with complex relationships and differing opinions considers the structural, human resource and perhaps political frames, which all deal with leadership structure, empowerment dynamics and the conflict or forging of relationships. Disjointed and misaligned university departments with little to know communication might experience this phenomenon if they are serving the same or similar audiences or are competing for the same scarce resources; it becomes chaotic and when it gets so bad, constituents are forced to collaborate for a solution, but it may only be episodic to calm internal or external pressures. Along these lines, Weick and Quinn's (1999) view of the episodic change process from an analytical perspective dealt with time/inertia. They contend that episodic change is usually not fully implemented, is slower to occur and that people will begin to get frustrated and stage a revolution when the change process and adaptation of solutions lags. According to Weick and Quinn's work, inertia was defined as the "inability for organizations to change as rapidly as the environment" (Pfeffer, 1997, 163) which may be attributed to deep structure (Gersick, 1991), first-order change (Bartunek, 1993), routines (Gioia, 1992) success-induced blind spots (Miller, 1993), top management (Virany et al, 1992) identity maintenance (Sevon, 1996), culture (Harrison and Carroll, 1991), complacency (Kotter, 1996), technology (Tushman and Rosenkopt, 1992) etc. Whatever the reason for the inability of 
organizations to change quickly, Romanelli and Tushman (1994) proclaimed the need for a revolution to change "a system of interrelated organizational parts that is maintained by mutual dependencies among the parts and with competitive, regulatory and technological systems outside the organization that reinforce the legitimacy of managerial choices that produced the parts" (p. 114). In other words, when an organization has strong ties to its current structure and behaviors due to the interconnectedness of their practices and other influences, its leadership will not be swayed by light-hearted suggestions for changes; it must be a revolution to bring attention to the peoples' demands and begin to value an organization's people, structures, and practices and more than peripheral to success.

On the other hand, to briefly summarize the metaphors to describe continuous change, organizations would need to engage collaboratively with constituents in ongoing improvisation (Inventiveness) and executions of work practices and polices etc. Additionally, constituents should repeatedly translate changes in a way that converts ideas into useful pieces that align with the intended purpose and engaging in continuous learning to increase, strengthen or reduce the repertoire of responses to determine the best form of action. Weick and Quinn (1999) believed that continuity and scale are where the most issues with continuous change occur and that continuity issues are associated with organizational culture (Trice and Beyer, 1993). The notion is that culture clasps a number of changes together and gives validation to nonconforming actions that improve the ability for things to be reworked and grounds the understanding of this reworking into the norms and values (Kotter and Heskett, 1992; O'Reilly and Chatman, 1996). Colville, Dalton, and Tompkins (1993) declared "If we understand culture to be a stock of knowledge that has been codified into a pattern of recipes for handling situations, then very often with time and routine they become tacit and taken for granted and form the schemas which drive action" ( $p$. 559). To this end, culture becomes a standard of knowledge that serves as an outline for how people will measure their expression, actions and evaluation of actions. In other words, when one changes the often uninterpreted culture and analyzes language paradigms, it changes the climate (Schneider, Brief, Guzzo, 
1996; Colvill et al., 1993). When scale is considered in continuous change efforts, some scholars may believe that micro-level changes that address the nuances in processes, policies, behaviors etc. are too small to make a difference or be of much importance. On the contrary, other scholars have contended that small changes are not trivial, nor do they stay small but when occurring on the edge of chaos, can be transformational and ready for institutionalization (Staw and Sutton, 1993, Staw, 1991; Maruyama, 1963; Colville et al., 1993). Weick and Quinn (1999) suppose that a serious oversight to small, incremental or micro-level changes exists when they are overshadowed by large scale revolutions that often get the credit for being the only catalyst for revolutionary changes. Nonetheless, insight into intervention theory in both episodic and continuous change would be helpful to study for a better understanding of the intervention process. As the demands of Black students across the nation continue to rise on college campuses to form interventions, I find it vital to briefly synthesize a portion of the vast literature related to intervention and implementation.

\section{Intervention Theory and Implementation}

In Marshak (1993), Lewin (1951) proclaimed "to break open the shell of complacency and selfrighteousness it is sometimes necessary to bring about deliberately and emotional stir-up" (p. 400), which implies the need for revolutionary measures to achieve revolutionary organizational change. While Gersick (1991) considered this emotional approach fuel to energy, Barr and Huff (1997) and Driskell and Salas (1996) warn that the revolutionary passionate approach might cause strain and stress on the cognition and performance of those fighting for intentional change. Ford and Ford (1995) help us to understand that intentional change is realized by a change agent who intentionally and knowingly seeks to create circumstances and environments different from how they exist and next fulfills this goal by using a set or series of actions and interventions unaccompanied or by collaborating with others. In doing so, Lewin advocated for the unfreezing of group norms, personal defenses, and organizational culture. Schein (1996), in Weick and Quinn (1999), further this idea by providing a three-stage process for unfreezing, which are: 
(a)disconfirmation of expectations, (b) induction of learning anxiety if the disconfirming data are accepted as valid and relevant (e.g. the fear that if people admit to themselves and others what is discovered to be wrong or imperfect, they will lose effectiveness, self-esteem/identity), (c) provision of psychological safety that converts anxiety into motivation to change (p. 29). While not mentioned among the organizational change literature reviewed thus far, parallels can be drawn to these processes with the previous review of literature on identity development and intergroup dialogue. As people gain new cultural knowledge especially, it happens from various stages of unawareness to exploration and awareness; intergroup dialogue practices could make room for such cultural and social justice enlightenment. Moreover, Schen's (1996) work asserts that after these forgoing unfreezing processes that elicit change, cognitive restructuring occurs and causes words to be re-examined to redefine previously understood meanings and people establish new standards for judgement and evaluation. Additionally, during unfreezing people become more inclined to learn and become more focused on ideas that are in translation; thus, when refreezing happens, it implants the new behaviors learned until the next period of episodic change. However, Grimley, Prochaska, Velicer, Blais, and DiClementer (1994) conducted a study on weight loss and smoking cessation to view intervention for change from a different perspective. From this study, they presented for stages that people enter when exposed to change interventions; they are: precontemplation, contemplation, action, and maintenance. Pre-contemplators are oblivious to any need to change, contemplators are cognizant of the change needed, but have not committed to do so, those in action stage have modified their behavior and of course those in the maintenance stage is where people maintain their modified behaviors after spiraling back and forth through the stages during the intervention process (Grimley et al., 1994). If we apply these stages in a higher education context, we might find leaders and practitioners spiraling through these non-linear stages as new campus interventions are proposed or implemented; all constituents involved would do well to understand the somewhat cyclical nature of these stages and how people may move into action for a campus intervention, struggle with maintenance and 
return to contemplation before entering action again. To validate this assertion, Grimley et al.'s (1994) study reported that $85 \%$ of those who relapse returned to the contemplation stage but not as far as the pre-contemplation stage after action; meaning they are closer to re-entering action than not. Given the complexity of organizational change and development, people often look to or are called out by a person known as a change agent or prime mover.

The change agent is one who is the prime mover to bring about change in an organization and is tasks with neutralizing the tendencies of people in large groups for large-scale change. Such groups typically induce stereotyping, lack of ownership for ideas, more abstract constructs, and less willingness to share unique thoughts (Weick and Quinn, 1999). The prime mover-in this case-should neglect traditional organizational development assumptions by relying less on action or discrepancy theory and more on systems theory; by using less internal data and more data from the environment that can be shared broadly; by focusing more on a mixed model management approach to include both senior management and the organization in decision making etc. (Weick and Quinn, 1999). Moreover, Bate (1990) and O'Cononor (1995) inferred that language interventions were becoming a critical means for agents to bring about change. To support this idea, Rorty (1989) declared "a talent for speaking differently rather than for arguing well, is the chief instrument of cultural change" (p.7) and Bartunek (1999) add to this idea by claiming that a strong alternative schema should be presented clearly and persistently to produce second-order change. Other scholars have advocated for "cultural consciousness raising" by meeting with actors or smaller units of organizations to interpret their actions alongside a particular cultural purpose, meaning or history rather than combating their actions with technology, data, or feelings of incompetence (Wilkof, Brown, and Selsky, 1995). Moving along the lines for intervention theory, but with continuous change, a modification to the previous unfreeze, transition, and refreeze approach in episodic change was discovered. 
Intervention theory in continuous change follows a freeze, rebalance, and unfreeze approach. Unlike episodic change, the focus is on redirecting what is already in progress, of which Marshak (1993) developed six assumptions from a Confucian and/or Asian perspective. The assumptions are (a) cyclical (rise and fall patterns repeat themselves); (b) processional (movement consists of a logical sequence cycle, but departures cause imbalance); (c) journey (there is no end spot); (d) equilibrium (interventions restore balance); (e) appropriateness (precise action maintains harmony); and (f) change (nothing will remain the same incessantly) (Weick and Quinn, 1999, p. 379). To expound upon this, freezing allows constituents to show visuals of what exactly is happening in the present; it is reflective. Rebalancing requires organizations to reinterpret, relabel, restructure issues to create opportunities, respond to and address injustice, use appreciative inquiry to reinterpret historical dilemmas etc. And to unfreeze is to recommence improvisation, knowledge transformation, and be more considerate of sequences or systems when learning (Weick and Quinn, 1999). Leadership in continuous change focuses on the use of a logic of attraction; a counterpart to the logic of replacement in episodic change, whereby change happens in people and organizations when they are attracted, drawn, and inspired by it. Kotter (1996) contends that in order to lead change, one must show people how to be (to attract them) rather than manage change by telling people what to do (to replace what was). Thus, Weick (1995) stated the role of the change agent in continuous change must aid in the understanding of change dynamics already in progress; they bring attention to and reframe emergent changes, explicate where current upheavals are going and the potential outcomes of their redesign. In doing so, much like Freire (1970/1993) Schein (1993) advocated for dialogue in the face of creating new knowledge for changed conditions. For example, Schein (1993) believed that dialogue empowered groups of people to generate a shared set of meanings and a universal thinking process and stated, "the most basic mechanism of acquiring new information that leads to cognitive restructuring is to discover in a conversational process that the interpretation that someone else puts on a concept is different from one's own" (p. 31). According to Weick and Quinn (1999), J Quinn 
(1996) add to the strategic change idea with the assertion that "good conversation is vocal, reciprocating, issues-oriented, rational imaginative and honest," (p. 381). Likewise, Barrett, Thomas, Hocevar (1995) and Dixon (1997) proclaimed that everyday conversations is a tool to produce the most powerful change interventions. Higher education constituents would benefit from these scholarly recommendations in their attempt to reform campus climates by implementing various interventions for change. In addition to what is now known about organizational change/development, reframing organizations, and intervention theory, consider the process literature for the multiplicity of phases for implementing change along with the scholarship on how colleges change.

Armenakis and Bedeian's (1999) work synthesizes a wealth of literature pertaining to the vast research conducted on implementing change for change agents to consider. First, Judson's (1991) five phase model for implementing change is comprised of: (a) analyzing and planning the change; (b) communicating the change; (c) gaining acceptance of new behaviors; (d)changing from the status quo to a desired state; and (e) consolidating and institutionalizing the new state. Given the reality that change efforts are likely to cause resistance, Judson (1999) predicted reactions to the phases of this change model and ways to minimize resistance through alternative media, bargaining and persuasion, and reward programs (Armenakis and Bedeian's, 1999, p. 301). Contrastingly to Judson's (1991) model, Kotter (1995) presented eight steps for change agents: (a) establishing a sense of urgency by relating external environmental realities to real and potential crises and opportunities facing an organization; (b) forming a powerful coalition of individuals who embrace the need for change and who can rally others to support the effort; (c) creating a vision to accomplish the desired end-result; (d) communicating the vision through numerous communication channels; (e) empowering others to act on the vision by changing structures, systems, policies, and procedures in ways that will facilitate implementation; ( $f$ ) planning for and creating short-term wins by publicizing success, thereby building momentum for continued change; and (g) consolidating improvements and changing other structures, systems, procedures, and policies that are not 
consistent with the vision; and ( $h$ ) institutionalizing the new approaches by publicizing the connection between the change effort and organizational success (Armenakis and Bedeian's, 1999, p. 301). While the two models have some similarities, Kotter's (1995) work delves a bit deeper in its step by step process during to process of producing successful change efforts. Moreover, Armenakis and Bedeian's (1999) also suggests Galpin's (1996) nine wedge wheel model, of which I find to be similar to Bolman and Deal's (2008) work by considering an organization's culture, norms, ceremonies and events when implementing change. Additionally, as previously referenced in our explanation of continuous change, Trice and Beyer (1993) validate the importance of culture in change work as in Galphin's nine-wedge model which calls for: (a) establishing the need to change; (b) developing and disseminating a vision of a planned change; (c) diagnosing and analyzing the current situations; (d) generating recommendations; (e) detailing the recommendations; (f) pilot testing the recommendations; (g) preparing the recommendations for rollout; (h)rolling out the recommendations; (i) measuring, reinforcing and refining the change. As one my gather from the previous model, it takes a more cautious approach by gathering recommendations and pilot testing before a large-scale rollout is implemented. Such precautions here and with aforementioned models are mindful of potential resistance to change and thereby seek to minimize it through its theoretically driven approaches. Armenakis, Harris, Field (1999) drew from Bandura's (1986) social learning theory and Lewin's (1947) work to put forth two more models concerned with minimizing resistance and the other to adopt and institutionalize the desired change. First, Armenakis and Bedeian's (1999) synthesis of process literature stated that Armenakis et al., (1999) argued that to be effective, change agents should include five components: (a) discrepancy (i.e., we need to change); (b) self-efficacy (i.e., we have the capability to successfully change); (c) personal valence (i.e., it is in our best interest to change); (d) principal support (i.e., those affected are behind the change); (e) appropriateness (i.e., the desired change is right for the focal organizations) (Armenakis and Bedeian's, 1999, p. 302). Second, as previously noted in, Bate (1990) and O'Cononor's (1995) assertion for language intervention, Armenakis et al., (1999) put 
forth strategies to aid change agents in their transmittal of change messages: (a) persuasive communication (e.g., speeches by change agents and articles in employee newsletters); (b) active participation by those affected (e.g., vicarious learning, enactive mastery, and participative decision making); (c) human resource management practices (e.g., selection, performance appraisal, compensation, and training and development programs); (d) symbolic activities (e.g., rite and ceremonies); (e) diffusion practices (e.g., best practice programs and transition teams); (f) management of internal and external information; and (g) formal activities that demonstrate support for change initiatives (e.g., new organizational structures and revised job descriptions) (Armenakis and Bedeian's, 1999, p. 302). Much of the literature I discovered on models for implementing change will be useful for change agents to dwell on and use as guides for their aspirations to implement interventions within the higher education context. Given the overwhelming amount of campus unrest and student demands for change efforts within the campus climate and ecology, change agents will increase the likelihood for successful change outcomes if they utilize the recommended models and are prepared for minimizing and managing resistance, which is bound to happen during campus revolutions. Albeit the scholarship of the 1990 s contains an overabundance of theoretical gems to employ within organizations in general, consider how colleges change today.

\section{How Colleges Change}

Kezar (2018) is among the plethora of theoreticians who focuses her work on understanding, leading and enacting change, but more specifically within the higher education context. I highly recommend her text, How Colleges Change, to any prime mover seeking to bring about change on their respective college campuses because of its specificity to the profession and the empirical knowledge used to inform her work. Although, I find Kezar's (2018) work to be an exceptional guide and resource for higher education practitioners/educators, I do not attempt to present or expound upon the girth of her work in this dissertation due to space limitations but have tried to ascertain some of the most salient information 
to this study. As I bring this topic forward in the context of today's campus climate, Kezar (2018) presents several reasons as to why the environment for change is different today; they are:

1. Connection of higher education to the global economy

2. The greater public investment and sense of accountability

3. Increasingly diverse students who engage campuses differently

4. The corporatized higher education environment

5. For-profit-higher education, competition, and marketization

6. New knowledge about how people learn

7. Technology

8. Internationalization of campuses

Kezar (2018) contends that while these factors reinforce the need for change, we should also realize how they change the context for change and begin a new era whereby change occurs; thus, context matters. Today, more students than ever are being educated by higher education institutions, but because all students are not the same, practitioners must consider the needs of the various types of students served (e.g. first generation, adult learners, physically challenged, and a greater diversity of racial, ethnic, socioeconomic, gender, and international populations) (Kezar, 2018, p. 9). Despite the increase in the diversity of students on campuses today and the growing number of Hispanic students, Kezar (2018) calls our attention to the low retention and graduation rates for low-income and racial minorities. In fact, the 2000 Census reported 16 percent of all Hispanics, 21 percent of all African Americans, 15 percent of Native Americans earned a college degree, compared to 35 percent for Caucasian and 49 percent of Asian Americans. Additionally, the graduation rates for Hispanic and African American students are 45 and 31 percent respectively, while Caucasian and Asian American student rates are 58 and 65 percent respectively at Division I colleges. Thus, low academic persistence and completion rates coupled with the increase in in diversity among faculty have shaken up traditional campus practices, communications and ways of 
thinking that were more aligned among faculty and staff of the same racial and social backgrounds. The increase in diversity has made the creation of a shared vision more complex and in some instances, change agents are ill-prepared to listen, understand unfamiliar perspectives, and include people from diverse backgrounds in the complexities of decisions along the change process. (Kezar, 2018, p. 10) Nevertheless, these issues, among many other faculty and administrator power dynamics etc., are met with challenges for remedy in a corporatized higher education climate where leaders across institutions are less collegial in sharing information because they view themselves in competition with other schools. (Kezar, 2018, p. 12) Another issue today is around new knowledge and how people learn; Kezar (2018) argues that traditional teaching practices that present information abstractly is counter-productive when a growing body of research shows that students learn better when explicit connections are made across content areas and by scaffolding their existing knowledge. Likewise, Kezar (2018) contends that studies reinforce a need for practitioners to rethink curricular structures, pedagogical practices, values, the architecture of classroom space, and the student-teacher relationship. To address these findings, campuses are shifting towards senior capstones, learning communities, and problem-based and experiential models that draw from students' interests and emotions through service learning, self-created majors and student-driven assignments (Kezar, 2018, p. 14). Even in terms of the internationalization of campuses, Kezar (2018) advocates for student preparedness in global perspectives and international experiences as a result of a reexamined curriculum and pedagogies. With this in mind, Kezar (2018) informs change agents that people often fear change because of a lack of understanding of the change itself and not just their own attachment to the status quo, but that sensemaking and organizational learning is important to swing the pendulum away from resistance towards change efforts. Moving forward with the importance of thoroughly understanding today's current context of issues, is the public purpose versus corporatization context. Kezar (2018) highlight the revenue-generating and corporate framework of higher education institutions and warns change agents against sacrificing one for the other by moving steadily towards 
financial gain at the expense of critical aspects of the institutional mission. In fact, in spite of the current revenue-generating and corporate context, Kezar (2018) states "It takes a very courageous and systemic style of leadership to recognize the substantial pull of the revenue generation, while also understanding the need to emphasize the mission and priority of the public good...there is a need for advancing a change agenda that has much broader scope, tying individual changes to a broader vision for the future of the enterprise" (p. 19). In other words, Kezar (2018) believed that the quality of curriculum and the incorporation of innovative pedagogies and teaching approaches, along with how well students are supported towards completion, improve higher education. In light of this belief, Kezar (2018) asserts the critical impact that addressing diversity and equity issues can have on the sense of community and relationships of any campus; thus, it would be beneficial for change leaders to find a balance to reap the benefits and rewards of an improved campus racial climate and have fiscal stability. To do so would ensure that a reasonable focus is placed on a continuously improving experience of those who will benefit from change.

Along these lines, Kezar (2018) asserts the notion of ethics in change and its place as a primary consideration for change agents given the likelihood of resistance to ill-conceived changes university officials may face. Thus, Kezar (2018) stresses the importance of examining the beneficiaries of any proposed change, determining whose interest will be served and being vigilant with identifying ethical situations to yield choices in support of the greater good. But while making ethical decisions for the greater good is recommended, Kezar (2018) reminds us to use caution if considering Kiddler's (1995) maxim: "the greatest good for the greatest number," (p. 26) because serving the broader good, in some cases, can hurt minority groups; especially when their specific and uniquely different needs are not being fully considered. For example, Kezar (2018), argues that in her 20 years on college campuses, there has been little to no discussion about ethics of change initiatives; therefore, campus leaders have failed to allow campus stakeholders to inquire as to whether the institution's pursuit for corporatized prestige etc. 
undermine the institutional goals for student learning outcomes. As a result, Kezar (2018) states that "the question of whose interests are served by a change and who loses out needs very careful consideration from change agents...changes that put undue stress on those in marginalized positions or work against their interests should be viewed with suspicion, compared to those that might decrease the interest of those in power" (p. 28). In other words, Kezar (2018) harps on the fact that whether a change benefits the client or not is always important and that because students are the primary concern and beneficiaries of educational institutions, their interests should be the ultimate interest being served by any change initiatives. Another ethical dilemma to consider is when change agents attempt to exclude the people they believe will resist or challenge the problems they have with a proposed campus reform/change initiative. This unethical method gives agents a chance to begin implementing their reform efforts before criticism and resistance is able to emerge; whereby missing the opportunity to rather create room for buy-in to avoid resistance and cynicism (Kezar, 2018). Mabin, Forbeson and Green (2001) validate this idea in their studies that show leaders who sought to obtain input from resisters and cynics who were better able to adjust and strengthen their original plans, gain buy-in and even relinquish poor change initiatives. Kezar (2018) show how Dean, Brandes, and Dharwadkar (1998) also support this idea by noting that cynics can essentially become a conscience or moral compass for an organization given their willingness to be outspoken when other might be afraid; they may also be more likely to engage in dialogue unlike active resisters. To properly frame this idea, resistance is described by Kezar (2018) as a positive force for change agents to welcome in ongoing dialogue rather than avoiding and overcoming it as previous literature has suggested. However, Kezar (2018) shares Thomas, Sargent and Hardy's (2011) critical assessment, utility and precautions when engaging resistance; they corroborate the productive nature of it, but only when it is constructive. This important assessment by Kezar (2018) from Thomas et al. (2011) asserts that ...there are forms of resistance that do not lead to better outcomes. When people are constructive in their resistance, they have become unilateral and are often no longer willing to listen to to 
engage in two-way communication. The authors caution that many poor change processes can leave organizations depleted and incapable of engaging in this type of positive dialogue. Still, the generative dialogue and communication may help with creating a better atmosphere that can foster change down the line, even if the current initiative fails (p. 35).

Of importance, I draw similarities in the seminal work of Paulo Freire $(1968 / 1993)$ and other theoreticians presented in this review of literature around the critical nature and need for dialogue. Given the current campus racial climate issues, protests and student demands highlighted from the literature, it would be crucial for change agents to create opportunities for diverse groups of people to collaborate on proposed plans, submitting alternative plans and creating more synergy around campus reform efforts. Abandoning these recommendations for a top-down change implementation approach causes violations to organizational fairness and justice (Kezar, 2018). To avoid these pitfalls, Kezar (2007b) study found that college presidents who engaged regularly with students of color, learned from them, and consequently advanced their diversity agendas. Kezar (2018) supports this idea with the assertion that stakeholder participation in change efforts should be authentic and that people in power should include the opinions of all stakeholders in their planning or run the risk of needing to explain why their feedback was ignored. Therefore, employees must not only trust a leader, but believe in their ability to bring about change. For these reasons, change agents should consult Kezar's (2018) multifaceted framework for understanding change.

The multifaceted framework for change was developed by Kezar (2018) and includes four macro areas: (a) type of change, (b) context for change, (c) Agency/Leadership, (d) approaches to change (See Figure 4). Each area consists of various features to reference to help people understand the change process, how external and organizational context shape success or failure, available leadership to develop change, and the characteristics of the change initiative as it pertains to fit inside the organizational culture and structures (Kezar, 2018, p. 66). Given space limitations, I will not address all features, but recommend 
change leaders use this framework to guide campus reform efforts. However, one example feature that falls under type of change is content of change; depending upon what is being considered for change, agents must consider the dynamics they may face because of the content of the change.

\section{Figure 4}

Change Macro Framework

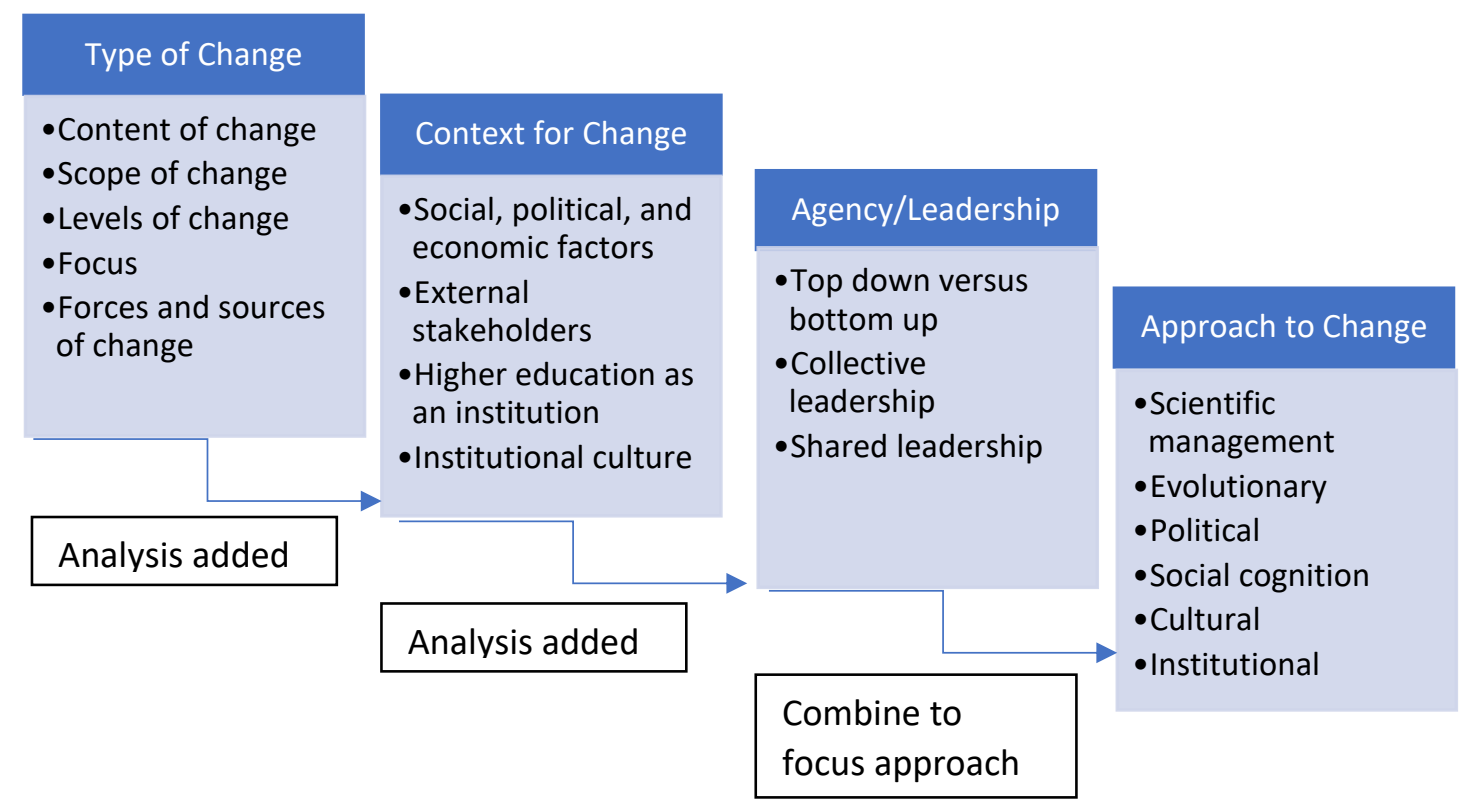

Note. Kezar, 2018

For example, Kezar (2018) contend that multiculturalism, unlike technology where everyone accepts it to be an inevitable part of the workplace, is not always the interest of White faculty, staff and students as it is for faculty, staff and students of color. Naturally, given the indifference to be concerned with multiculturalism, there is bound to be more resistance towards change efforts that do not appeal to others' interests. Kezar (2018) asserts that "multiculturalism often requires deep shifts in thinking and learning, requiring people to undergo double-loop learning in order to fully appreciate the concepts. Double-loop learning is harder and less common because it calls for people to questions underlying principles. Multiculturalism involves understanding new cultures, recognizing privilege and power, and 
rethinking ones' view of the world" (p. 70). To expound upon this claim, Kezar (2018) situates multiculturalism and technology in a story of a higher education practitioner who is more successful at implementing new technology efforts that she is with multicultural initiatives. The text suggest she work with campus administrators garnering their support to multiculturalism by reframing the campus mission and vision-among other suggestions. Additionally, in moving toward the context of change area of the multifaceted framework, Kezar (2018) includes institutional culture as a feature to consider, so that institutions understand the history of the institution/department and whether or not its values are aligned with institutional practices that support or prevent change or if academic freedom or shared governance is a part of the culture. Agents who do their research to understand the dynamics and previous concerns can avoid being blindsided by unknown historical perspectives. Kezar (2018) warns that agents who try to implement universal or campus wide policy change on a decentralized campus might cause resistance, but if the change leader works from the bottom up with different departments to gain buy-in and support for new policies or initiatives, might see the light of day campus-wide. In line with institutional culture are an institutions values and whether the campus mission and values statements are more aspirational for what they hope to be rather than a reflection of daily actions that align with said statements. Likewise, Kezar (2018) recommend change leaders speak with and monitor the actions of people to determine if campuses are in support of multiculturalism. To this end, Kezar (2018) cautions agents to "not be fooled by manifestations of espoused values, which often lead individuals to believe that changes are going to encounter greater success and less resistance," and that conversations with individuals across campus along with observed behaviors and an analysis of campus artifacts help to determine the campuses' "actual" value system. In order for campuses to change, Kezar (2018) argues that organizational capacity and readiness for change are critical to the success of changes and need to be strong; in fact, she lists Toma's (2010) outline for smoothly participating in the change process:

- A clear and meaningful mission 
- Structures that support the mission and vision

- Healthy governance processes

- Polices that support institutional mission and vision

- Streamlined and clear processes

- Healthy information resources that are well shared

- Facilities

- Technology

- Human infrastructure

- Capital assets that are continually maintained and updated

- An institutional culture that promotes the predominant values of the mission and vision ( $p$. 130).

These areas listed above, reside under elements of the context of change feature, are not an exhaustive outline of all change dynamics but should be considered among the many other elements attached to each feature within the multifaceted framework for change. Albeit, the outline covers a general perspective on context for the areas change agents may begin strategizing from, Kezar (2018) provide a number of intricate assumptions about the type of leadership needed and their agency for change; this is the next area in the framework to address.

The most salient among the literature on leadership and agency of change fitting our interests are negotiation skills and skepticism and suspicion. Negotiation among administrators and other people within the ecosystem is necessary because administrators may not always agree to issues or demands for initiatives, so Kezar (2018) recommends advocates for change ask for more than what they really need to account for the percentage of the request that administrators are bound to deny. Doing so would likely end in change that is just enough or more that needed, but those seeking change must remain flexible as well so that all desires for change do not come to a complete halt. This is an important recommendation to consider for our study in particular because of the numerous demands Black students have made across the nation. Kezar (2018) provided two examples in her text where group of students asked for an 80 percent reduction in the campus' carbon footprint when they only needed 50 but the university agreed to a 65 percent reduction. In another example, after the faculty refused to negotiate on their request for 10 new faculty hires, the administration decided not to hire any new faculty; as a result, Kezar (2018) warns 
that some grassroot leaders not willing to negotiate may be shunned and their initiatives not embraced by top-down leaders. The particularism of skepticism and suspicion bold well for our study as it speaks directly of administrators/top-down leaders who-in many cases-do not share the same interests as grassroots leaders, especially diversity. Kezar (2018) infer that top down leaders may focus on diversity by addressing community needs or improving retention rates, whereas, bottom-up faculty and staff leaders are concerned with a broad-based understanding of diversity and equity on campus along with raising awareness of White and Class privilege. The faculty and staff in this example were interested in changing the curriculum and leaning experiences by mixing materials and lesson that more accurately reflect their understanding of diversity. Moreover, Kezar (2018) suggests grassroots leaders not be fooled by false rhetoric of top-down leaders and try to approach such massive change efforts with skepticism and suspicion. Finally, on this idea, Kezar (2018) shares how one change agent who carefully ignited the need for a particular change effort among colleagues, however, became a focal point for criticism which limited her ability to be effective; thus, she reached out to other faculty and student groups to rally around her efforts and begin to push them from where they are by using their collective power. For example, student groups can address more radical ideas, cluster hiring, curriculum changes etc.; things that are much too controversial for one change agent to bare alone. To address the fourth area of the multifaceted framework called approach to change, I urge change leaders to view Kezar's (2018) work for an understanding of the six schools of thought in their entirety; they are: scientific management, evolutionary, political, social cognition, cultural and institutional. Next, I wrap this massive review of literature up by citing Kezar 's (2018) work on change implementation. While I have discussed a wealth of literature on change for campus reform, we must be concerned with the sustainability of the change being implemented through institutionalizing change.

Therefore, Kezar (2018) references a change institutionalization framework to guide thoughts on institutionalizing the change processes. According to Curry (1992) and Kramer (2000), to institutionalize is 
for change efforts to become a part of the day-to-day operations with an established standard within the campus' human ecological system; change becomes more permanent, expected, widespread and routine (Kramer, 2000). Thus, a three-stage model of change was put forth; they include:

1. Mobilization: "the system is prepared for change"

2. Implementation: "the change is introduced"

3. Institutionalization: "the system is stabilized in its changed state."

(Curry, 1991, cited in Curry, 1992, p. 5)

To this end, after moving through the above stages, institutionalization should be realized and achieved when the change become a part of the overall value system of the organization and is no longer seen as innovative, but rather commonplace for how things just are (Kezar, 2018). Students, faculty-administrators and all the constituents of any campus reform efforts or those who put forth demands to top-leaders, are strongly encouraged to consider work collaboratively together and use dialogue to guide the journey towards campus reform. 


\section{Chapter Three}

\section{Methodology}

This chapter expounds upon the nature of inquiry and grounds the direction for conducting a Critical Race qualitative study using case study design methods. To investigate the discontinuities in race relations on a college campus, the student, faculty, and administrator perceptions and responses were collected to highlight the process, while they were making, developing, and implementing Black students' demands as interventions. Thus, a single case study method was used to understand the current contextual factors which ultimately impact organizational change in higher education. In doing so, the following has been addressed: (a) purpose of the research method used, (b) research questions, (c) research design, (d) site selection, (e) sample participants, (f) data collection, (g) data analysis, (h) research validity and (i) author's positionality.

Qualitative inquiry is concerned with understanding social and human problems through individual or group meanings. Emerging questions, procedures and collecting data in participant's settings most often are common research practices in qualitative studies. Inductive data analysis is built from gathered details that inform or become linked to general themes (Creswell, 2014). This inquiry, allows researchers to seek out the complexities of problems not often known, told or understood and to draw out meaning and empirical knowledge to come to understand complex social and human phenomena.

The purpose of this study was to explore the perceptions of the institutional response to an exceptional campus racial climate issue at the University of Missouri and the process of formulating and participating in a diversity training course and a semester long course centered around race. Additionally, this dissertation helps to understand how participants perceived this curricular intervention to have addressed the discontinuities in race and racism on campus and contributed to organizational change, and institutional reform. 


\section{Research questions:}

This research was guided by the following questions:

1. What are the perceptions of the process of formulating and participating in a curricular response to an exceptional campus racial climate issue at the University of Missouri??

2. What are the perceptions of how this curricular response addressed discontinues in race and racism on campus and contributed to organizational change and institutional reform at the University of Missouri?

A researcher's epistemology typically guides or ignites the nature of inquiry to explore. My ascribed epistemology as a researcher is constructivist and transformative. I ascribe to a constructivist epistemology, because it does not assume one single truth to form knowledge, but rather is subjectiveversus objective truth -in that individuals make meaning and socially construct knowledge. Using a constructivist worldview is important for this study for multiple reasons: Human beings construct meanings as they engage with the world they are interpreting. Qualitative researchers tend to use openended questions so that the participants can share their views, which was used in this study. Another reason, is that the rudimentary creation of meaning is continuously social, ascending in and out of interaction with a human community. As an inductive approach, I generated meaning from the data collected in the field. (Crotty, 1998)

To prepare for data collection, I took a moment to consider my overarching theoretical framework - Critical Race Theory/Intersectionality - for this study, to craft topics and semi-structured interview protocols able to produce responses that are in line with the foundations for my inquiry and interest in transformation and institutional change. Additionally, it is important to note that my constructivist and transformative epizoologies pair well together. In fact, DeCuir and Dixson (2004) contend that social change is implicit within CRT; therefore, those who use it must do so with caution to 
consider how their scholarship assists in the mission of social justice and social change. To validate this praxis, Patton (2002) proclaims that Critical Theory (A precursor/umbrella philosophy to Critical Race Theory) is one of the most influential orientational frameworks as it focuses on how injustice and subjugation form people's experiences and understanding of the world. Additionally, according to Kincheloe and McLaren (2000), a critical social theory deals with issues of power and justice and how the economy, issues of race, class, gender, ideologies, education, and other social institutions and cultural dynamics interplay to construct a social system. In fact, Kincheloe and McLaren (2000) espoused “...inquiry that aspires to the name critical must be connected to an attempt to confront the injustice of a particular society...Research thus becomes a transformative endeavor unembarrassed by the label political and unafraid to consummate a relationship with emancipatory consciousness (p. 281,291). This claim reinforces and confirms my philosophical worldview given its insistence for research to become transformative and leading to change for non-dominant groups; in this case, institutional/organizational change within the campus racial climate for Black students who demand it. In addition, Patton (2002) shares that the term critical is as such because it moves beyond the act of studying and coming to understand society but rather to critique and change society (p.131). Likewise, he cautions researchers espousing such theoretical orientations to remember that "the focus of inquiry is determined by the framework within which one is operating, and findings are interpreted and given meaning from the perspective of that pre-ordinate theory. Such qualitative inquiry, therefore, aims to describe and explain specific manifestations of already-presumed general patterns. Such inquiry is aimed at confirmation and elucidation rather than discovery" (p.131). Because of my inner desire to address social inequities with an action agenda, my epistemological ascription to constructivism is further advanced by the transformative worldview. The transformative worldview is intentional in its efforts to produce research with the ability to elicit change in the lives of marginalized people by using social justice, power, oppression, and inequality as a focal point to engage and give voice to participants. Again, this is a clear connection to the tenets of 
Critical Race Theory and Intersectionality. As a constructivist researcher, then, I am interested in the transformative outcomes that lead to racial and social equity. It is with these assumptions in mind that I reflected on my epistemologies as a constructivist and transformative researcher when I prepared to enter data collection and analysis within the scope of CRT and CRT/I frameworks.

\section{Research design}

This dissertation used a case study research design. According to Yin (2009) "A case study is an empirical inquiry that investigates a contemporary phenomenon within its real-life context, especially when the boundaries between phenomenon and context are not clearly evident" (p. 18). Merriam (1998) contends that the most defining characteristic of a case study is "delimiting the object of the study, the case" (p. 27). Of importance to this study, is Becker's (1968) definition of a case study's two-fold purpose; "to arrive at a comprehensive understanding of the groups under study" and "to develop general theoretical statements about regularities in social structure and process" (p. 23). Given this study's concern with Black students' demands of faculty and administrators involved in the event, inequities in the social structure and processes involved in the institutional response, a case study research design was appropriate. To illuminate the nature of a case study research design, Yin (2018) provided the second part of the definition of a case study by stating that it a technically distinctive situation where there will be a lot more variables of interest than data points, of which, leads to two results: the benefits from the previous development of theoretical propositions guiding design, data collection and analysis and the relying on of numerous sources of evidence with the need for data to converge by triangulation (p. 15). While there are likely to be more variables of interests than data points for this case, I relied on the use of my theoretical frameworks to remain focused on the purpose of the study while also relying on multiple sources of data as a form of triangulation to reinforce validity. While being fully aware of a nationwide campus racial climate issue and an uptick in Black student demands, I limited my inquiry to the curricular interventions 
and/or institutional reform efforts following the racial unrest and protests incidents of 2015 at The University of Missouri at Mizzou.

\section{Site Selection}

The location for this case study takes place at the University of Missouri (Mizzou) in Columbia Missouri. Established in 1839, Mizzou is a flagship land-grant institution, the first public university west of the Mississippi River and is known for starting the world's first school of journalism and homecoming as a tradition. It is one of four universities within the University of Missouri system, which also include: University of Missouri-Kansas City (UMKC), Missouri University of Science and Technology (S\&T), and the University of Missouri-St. Louis (UMSL); collectively representing one of the greatest assets in the state as a $\$ 3.0$ billion enterprise (History of Mizzou, n.d.; About the University of Missouri System, n.d.). As of fall 2018, MU or Mizzou, as it is often referred to, had 29, 843 students enrolled, 3,103 faculty, 13.804 staff and awarded 9,279 degrees in 2018. Comparing numbers from 2015 and 2018 allows us to see how things were at the time of the incidents and following the incidents, using the most recent information available in 2018. For enrollment by race in 2015, the main year of inquiry, Black/African American/non-Hispanic was 2,544 compared to 2,067 in 2018. White and non-Hispanic enrollment in 2015 was 26,921 compared to 22,506 in 2018 . As it relates to faculty, tenured, tenure-track and non-tenure track employed in 2015 , Black/African American/Non-Hispanic was 58 compared to 70 in 2018. For White and non-Hispanics in 2015, there were 1,503 compared to 1,446 individuals employed (History of Mizzou, n.d.; About the University of Missouri System, n.d.). Because we are coming to understand the perceptions and responses of those involved in the aftermath of the case through a CRT/CRT/I lens, it would be helpful to have some knowledge of what the university values. Mizzou's vision under the auspices of "what makes a Tiger" are:

1. Respect: We commit to acting ethically, welcoming difference and exchanging ideas openly.

2. Responsibility: We are accountable to ourselves, each other and the public we serve. 
3. Discovery: We foster the lifelong process of seeking knowledge and greater understanding.

4. Excellence: We reach for excellence through diligent effort and collaboration.

\section{(https://missouri.edu/about/history\#)}

With these institutional and geographical data facts, I examined the events of this case with them in mind as I sought to understand more about the campus racial climate and human ecological system issues which led to campus unrest worthy to spark national attention.

\section{Sample participants}

I selected participants for this study with a widely used qualitative technique known as purposeful sampling and snowball sampling. Creswell (2014) urges researchers to select sites and participants that will best help to understand problems and the research question(s) but limited in quantity that is typical in quantitative studies. Four aspects researchers might consider when selecting sites and participants are: (a) the setting (i.e. where the research will take place), (b) the actors (i.e. who will be observed or interviewed), (c) the events (i.e. what the actors will be observed or interviewed doing), and (d) the process (i.e. the evolving nature of events undertaken by the actors within the setting) (Miles and Huberman, 1994, p. 30; Creswell, 2014, p. 189). While Creswell (2014) espouses that there is not one set number of participants for each qualitative design, he infers that most case studies would have about four to five; however, saturation should be considered to avoid under or over-collecting data once the researcher achieves data saturation.

For this study, I selected participants from two categories: students and faculty/administrators. Students and faculty/administrator interviewees included participants who were directly or indirectly involved in the development of demands, protests, discrimination, intervention implementation or in opposition to student demands, their tactics, or the proposed interventions for reform. Given the centrality of race to this study, student and faculty participants selected were reflective of multiple races 
(Black, White, Hispanic, etc.) involved in the case and were officially affiliated with the university either at the time of the incident or presently. Race is indicated in the findings to account for any variation in racial dynamics among participants. Snowball sampling was used to help identify key constituents involved in the case and critical documents for data collection and analysis. Snowball sampling began as I was introduced to a University of Missouri administrator intricately involved and through my fraternal affiliation with individuals who connected me to people directly involved or recently/currently working to remedy and/or prevent the campus racial climate issues surrounding the 2015 incidents. Table 3 outlines the sample participants.

Table 3

Outline of Sample Participants

\begin{tabular}{|c|l|l|}
\hline \multicolumn{2}{|c|}{ OUTLINE OF SAMPLE PARTICIPANTS } \\
\hline \multicolumn{1}{|c|}{ Interview Participant } & \multicolumn{1}{|c|}{ Participant Affiliation/Role } & \multicolumn{1}{c|}{$\begin{array}{c}\text { Primary Purpose for } \\
\text { interviewing }\end{array}$} \\
\hline Key Interviewee 1 & $\begin{array}{l}\text { Professor and Department Chair } \\
\text { I Black Studies Department and } \\
\text { creator of both curricular } \\
\text { interventions. } \\
\text { Discipline: Black Studies and } \\
\text { Musicology } \\
\text { (Citizenship @Mizzou and Race } \\
\text { and the American Story) } \\
\text { provide pertinent perceptions } \\
\text { and information on the } \\
\text { development process of } \\
\text { curricular responses as the } \\
\text { creator and co-creator of both } \\
\text { interventions. }\end{array}$ & \begin{tabular}{l}
-Dr. Stephanie Shonekan \\
\hline
\end{tabular}
\end{tabular}


Table 3 (Continued)

\begin{tabular}{|c|c|c|}
\hline Interviewee 2 & $\begin{array}{l}\text { Co-Faculty creator of the Race } \\
\text { and the American Story course } \\
\text { Discipline: Political Science } \\
\text {-Dr. Adam Seagrave /Political } \\
\text { Science }\end{array}$ & $\begin{array}{l}\text { Faculty member who can } \\
\text { provide pertinent perceptions } \\
\text { and information on the } \\
\text { development process of } \\
\text { curricular responses as the } \\
\text { creator and co-creator of one of } \\
\text { the curricular interventions. }\end{array}$ \\
\hline Interviewee 3 & $\begin{array}{l}\text { Instructor of the Race and the } \\
\text { American Story course } \\
\text { Discipline: Political Science and } \\
\text { Black Studies } \\
\text {-Dr. Stephen Graves }\end{array}$ & $\begin{array}{l}\text { Can provide perceptions of the } \\
\text { course materials and impact on } \\
\text { students etc. from an } \\
\text { instructor's perspective. }\end{array}$ \\
\hline Interviewee 4 & $\begin{array}{l}\text { Student of the diversity training } \\
\text { course (Citizenship @ Mizzou) } \\
\text {-Jacob Somerscales }\end{array}$ & $\begin{array}{l}\text { A student who has co-facilitated } \\
\text { or attended the Citizenship } \\
\text { @Mizzou diversity training } \\
\text { course can give their } \\
\text { perceptions on the experience. }\end{array}$ \\
\hline Interviewee 5 & $\begin{array}{l}\text { Student of the diversity training } \\
\text { course (Citizenship @ Mizzou) } \\
\text {-Zoe Romyn }\end{array}$ & $\begin{array}{l}\text { A student who has attended the } \\
\text { Citizenship @Mizzou diversity } \\
\text { training course can give their } \\
\text { perceptions on the experience. }\end{array}$ \\
\hline Interviewee 6 & $\begin{array}{l}\text { Student of the Race and the } \\
\text { American Story course } \\
\text {-Luke Davis }\end{array}$ & $\begin{array}{l}\text { A student who has attended the } \\
\text { Citizenship Race and the } \\
\text { American Story course can give } \\
\text { their perceptions on the } \\
\text { experience. }\end{array}$ \\
\hline Interview 7 & $\begin{array}{l}\text { Student of the Race and the } \\
\text { American Story course } \\
\text {-Ida Campbell-Jones }\end{array}$ & $\begin{array}{l}\text { A student who has attended the } \\
\text { Citizenship Race and the } \\
\text { American Story course can give } \\
\text { their perceptions on the } \\
\text { experience. }\end{array}$ \\
\hline
\end{tabular}

\section{Data Collection}


After Institutional Review board (IRB) approval from both my host institution and being told approval was not needed from the case study site location - given its widespread national attention- I will used several qualitative data collection strategies to gather data from the case studied. Collection strategies included semi-structured interviews with guided topics and open-ended questions and document analysis. Brinkmann and Kvale (2015) contend that interview research is a craft that becomes an art when carried out effectively; they define the semi-structured life world interview as one "with the purpose of obtaining descriptions of the life world of the interviewee in order to interpret the meaning of the described phenomena" (p. 6). The qualitative interview is further described by Patton (2002) as one with open-ended questions and probes (follow-up questions) to gather in depth responses about the participants experiences, perceptions, opinions, feelings, and knowledge; thus, eliciting verbatim quotations with adequate context to be interpreted. Creswell (2014) adds to the options within this data collection strategy, which allow the researcher flexibility to conduct face-to-face, telephone, or group interviews with a few open-ended questions. Patton (2002) teaches us that the purpose of collecting responses to open-ended questions afford the researcher the opportunity to understand and capture the viewpoints of others without predetermining perspectives through apriori questions. The interviews for this study allowed me to collect and interpret participant responses that helped give meaning and understanding to the nature of inquiries made.

Next, documents collected were analyzed; they included the following: program records, university website correspondence, official journal publications, newspaper articles, a YouTube video, and responses to a course evaluation survey. (Patton, 2002). Creswell (2014) extends the notion of documents for analysis to qualitative audio and visual materials of relevance to a study, such as videotapes, website main pages, e-mails, text messages, social media text and other forms of sound.

For this study, I used semi-structured interviews beginning with case-relevant topics to get the story of reform to look for discrepancies and points of similarities and complexities during analysis. Virtual 
interviews were conducted and were video and audio recorded for subsequent transcription, analysis and to create a visual representation of the summary of findings. Participants interviewed were able to provide or suggest documents for analysis. I also continued to search the internet, university website and archives, local newspapers and media sources to gather materials to provide more unique insights into the case investigated. Table 4 is a list of documents analyzed.

\section{Table 4}

\section{Outline of Sample Documents}

\begin{tabular}{|c|c|}
\hline \multicolumn{2}{|c|}{ OUTLINE OF SAMPLE DOCUMENTS } \\
\hline Type of Document & Name of Document \\
\hline Newspaper Article & $\begin{array}{l}\text { MU Chancellor Loftin announces new diversity } \\
\text { initiative }\end{array}$ \\
\hline News Article & $\begin{array}{l}\text { MU Chancellor } R \text {. Bowen Loftin announces } \\
\text { resignation after backlash }\end{array}$ \\
\hline $\begin{array}{l}\text { List of Black Student } \\
\text { Demands/Letter }\end{array}$ & $\begin{array}{l}\text { The heart of the case study examined. Helped to } \\
\text { know what to track and inquire about from } \\
\text { demand to intervention. }\end{array}$ \\
\hline $\begin{array}{l}\text { Public domain journal: } \\
\text { Faculty interview }\end{array}$ & $\begin{array}{l}\text { The Mizzou Meltdown: How a President Lost } \\
\text { Control }\end{array}$ \\
\hline $\begin{array}{l}\text { College of Arts and } \\
\text { Science Website }\end{array}$ & A\&S Faculty Approve Diversity Course Requirement \\
\hline $\begin{array}{l}\text { Application to make } \\
\text { course Diversity } \\
\text { Intensive. Campus } \\
\text { website }\end{array}$ & $\begin{array}{l}\text { Frequently asked questions: How do I make my } \\
\text { existing course diversity intensive? (Guidelines) }\end{array}$ \\
\hline Course Evaluation & $\begin{array}{l}\text { Summary of Student evaluations for Race and the } \\
\text { American Story, taught by Dr. Stephen Graves. }\end{array}$ \\
\hline YouTube Video & Loftin Response to Racial Slurs Video \\
\hline
\end{tabular}




\section{Data Analysis}

After the collection of data from semi-structured interviews and data analysis within the case study design, I lean to the scholarly work of Johnny Saldaña to guide the data coding and analysis process. Additionally, I compared the themes/concepts from Critically coded data-using values coding-with the tenets and propositions of CRT/CRT/I theoretical frameworks to investigate the centrality of race and racism. After reading the themes and comparing them against the framework tenets, I reported how the themes/concepts were interpreted from a Critical race lens. Here, I provide a brief overview of the basic structure found in Saldaña's manual for coding, followed by the steps I used to mimic for my analysis. According to Saldaña (2016) a code is "a word or short phrase that symbolically assigns a summative, salient, essence-capturing, and/or evocative attribute for a portion of language based or visual data" (p. 4). Likewise, Charmaz (2001) described the process of coding as a "critical link" between the data collected and their explanation of meaning. Vogt, Vogt, Gardner, \& Heaffele (2014) and Saldaña (2016) described a code as a construct generated by the researcher(s) to translate data, whereby each individual datum is assigned an interpreted meaning. Such meanings assigned by codes are later used to detect patters, make categories, develop propositions, assertions, theories, and other analytic purposes. Before this can begin, researchers must be familiar with the various code types and uses to determine those most appropriate for the study. Coding examples include but are not limited to: descriptive, In Vivo, eclectic, values, process, emotion, versus and many more. After coding types are selected, and applied to each datum, the researcher should consider reviewing and scrutinizing their first cycle of coding decisions to add another layer of reliability to the interpretations being made to capture participant meanings. Next, the inquirer should begin to search for patterns among the codes applied. Saldaña (2016) defines a pattern as "repetitive, regular, or consistent occurrences of action/data that appear more than twice" (p. 5) and Stenner (2014) expound upon this idea with an assertion that patterns deal with the relationship between unity and multiplicity; "A pattern suggests a multiplicity of elements gathered into the unity of a particular 
arrangement" (p. 136). Moreover, qualitative researchers seek patterns as steady indicators of humans' ways of living and doing to better comprehend the world and predictability of human behavior. Patterns become the trustworthy evidence for our findings as they reveal habits, salience, and what is most important to people. Of significant importance, the researcher must remember the initial purpose for inquiry and how what emerges can be used to advance knowledge; they must consider and determine the analytic lens, filter, angle, or philosophical worldview when coding during analysis. For example, if coding a statement addressing one's opinion about a particular race in society: (a) In Vivo coding concerned with maintaining the participants exact words might code as "no place," (b) an ethnographer using Descriptive coding that is concerned with the breadth of opinions by multiple participants might code as "immigration issues," and (c) a critical race theorist using Values coding might code the participant's statement as Xenophobia. For this study, it was most appropriate to code using Values coding methods to be in line with the CRT/CRT/I framework used to guide analysis. After coding, reapplying codes and searching for patterns, the researcher should codify, which is to "arrange things in a systematic order, to make something a part of a system or classification, to categorize... A process that permits data to be divided, grouped, reorganized and linked in order to consolidate meaning and develop explanation" (Grbich, 2013 in Saldaña, 2016, p. 9). In other words, because the coded data under analysis may share some characteristics, they can be grouped into categories or families of which can be determined by the researcher's tacit and intuitive wits to group based upon the data that "look alike" and "feel alike" (Lincoln and Guba, 1985, p. 347). Following these steps, are the steps toward themes, concepts and in some cases assertions/theories. Saldaña (2016) contend that "a theme can be an outcome of coding, categorization, or analytic reflection, but it is not something that is, in itself, coded..." (p. 15). Figure 5 is a streamlined codesto-theory model for qualitative inquiry. Once themes/concepts are selected they can be shared along with the researcher's assertions or new theory (if applicable) as a part of the findings. 


\section{General Overview of Analysis to a CRT/CRT/I Specific Analysis}

Next, I have provided a general overview of my analysis, which included general qualitative analysis strategies that was also used with the applied CRT and CRT/I tenets to the data as a method for analysis. Second, I will provide a breakdown of how my overarching theoretical framework, CRT and CRT/I, has been used in previous studies and how I used it for this study.

\section{General Overview of Analysis}

In my analysis, I entered the process with a critical race theory and transformative lens. I examined the data collected, jotted down important details and initial discoveries as analytic memos. Next, I assigned codes using the framework of Values coding, which is suggested by Saldaña for Critical Race theorists, to symbolize and interpret participant responses and document data that form their own meanings but applied the CRT and or CRT/I tenants or analytical assumptions as codes. I used the analytic memos to double check and determine if initial memos were consistent with the codes applied. I then sifted through a second cycle of the data to confirm or re-code data to accurately reflect interpretations made initially. I codified the codes into categories or families based upon their characteristics in common, which seemed to have emerged naturally during coding. After categories of coded data were grouped, I applied themes/concepts to the categories with propositional statements to define the type of data that could appropriately fall under said themes. Where necessary, sub-codes and sub-categories were made to break out important aspects of the data needing to be highlighted. Based upon the themes/concepts that emerged, I was able to make or form assertions/a framework of considerations model to best articulate the transformative and practical nature of my findings, while drawing connections to the CRT/CRT/I tenets. 


\section{Figure 5}

A streamlined codes-to-theory model for qualitative inquiry

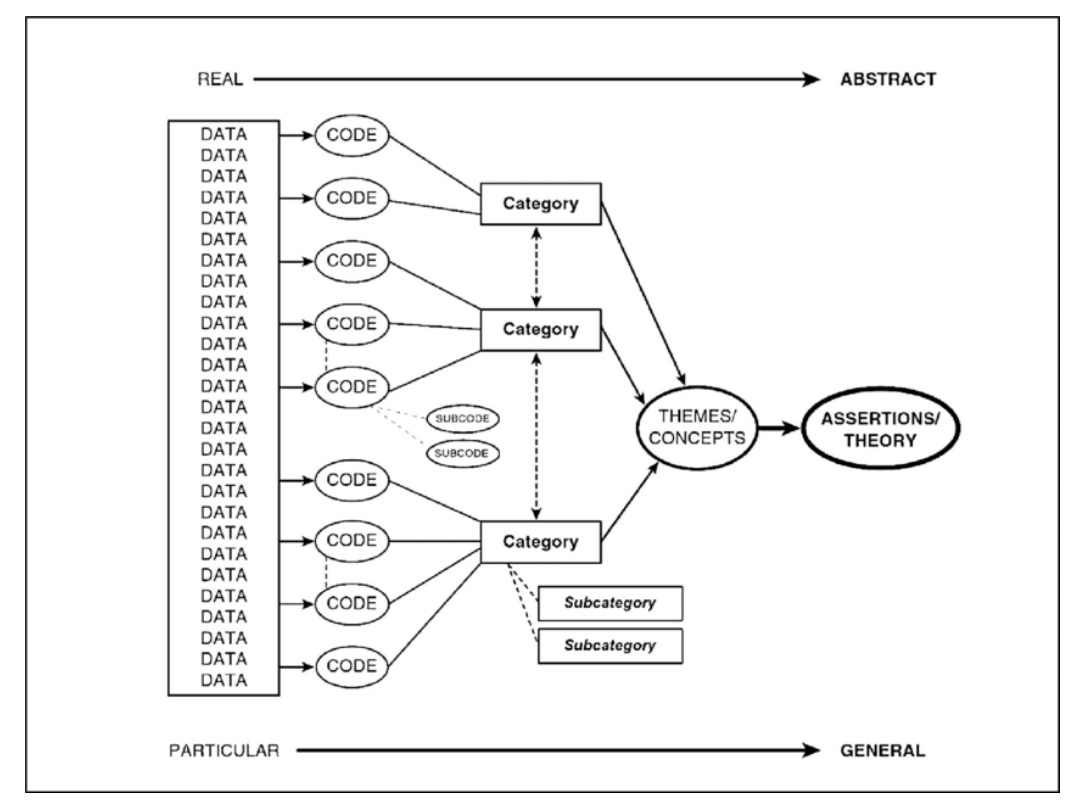

Note. Listed as figure 1.1 in Saldaña, 2016, p. 14

\section{The Use of CRT and CRT/I for Analysis}

I searched the literature to find a CRT framework for analysis, but there is not one set way to analyze data using CRT as a framework. Given that there is a gap in the methods, specifically the "how to" of CRT writing (Cook and Dixson, 2013), some researchers and scholars have made their own framework or used similar analytical strategies of other scholars to analyze their data using CRT, CRT/I and other critical race scholarship. I followed suit by somewhat modeling my critical race analysis after another scholar, but to fit my study, as I have been reflective about the kind of results I hope to present in the findings. First, I will briefly reference the works of a few scholars who used CRT or CRT/I as an analytical tool for analysis. DeCuir and Dixson (2004) use the five tents of CRT: Counter-storytelling, The Permanence of Racism, Whiteness as Property, Interest Convergence and Critique of Liberation to analyze race and racism in education. In their study, they tied various participant responses to at least one of the tents of CRT and was able to interpret the meaning of the data by framing it in the context of the chosen tenet. In another 
example, Cook and Dixson (2013) uses CRT as a method to analyze composite counter-stories on the experiences of Black teachers in New Orleans, post-Katrina. One of the authors listened to interviews, took additional notes, and created themes from interviews regarding pre- and post- Katrina school related experiences. Next, after coding the data pre-, post- or both, she dug through the data to determine what questions were answered or addressed and three themes emerged: loss/anger, isolation and the importance of education. She returned to the historical/temporal codes (pre/post/both Katrina) to overlay the three themes over the codes to advance the thematic story that emerged from participants. The thematic stories provided a foundation for the composite counter-story she wrote in subsequent sections. The author analyzed the themes that emerged to determine if and how they correspond with tenets of CRT, particularly counter storytelling. To create characters in the composite story, the author used larger CRT theoretical understandings of race and racism, of which connect in ways to the participants feelings of loss, anger, and isolation. This author used a CRT methodological framework to create counter-stories from interviews, professional and personal experience, and literature. One other example of CRT's use in analysis stems from two University of Missouri administrators who used duoethnography to conduct an analysis of their recorded pláticas (detailed stories of their experiences) during campus unrest (McElderry and Hernandez Rivera, 2017). CRT and Critical Race Feminism (FemCrit) were used to shape the analysis by allowing the researchers to interpret their findings through a framework that grounded their stories and experiences in a way that would challenge dominant structures and beliefs in effort to decide how ideologies and institutions can become more equitable. Experiential knowledge, which extends from the notion of a counter-story, is among the tenets of CRT; thus, this tenet was woven throughout their discussion of the themes that emerged and how they interpreted them. For example, they used to CRT's centrality on race and racism to discuss their feelings of isolation among colleagues who failed to recognize that the administrator's response was to a hostile environment and not their own character flaws; such preoccupation with racially coded assumptions spoke to the centrality of race and racism within CRT. The 
final example will be an example of how I used CRT and CRT/I to analyze my data. Chapter one outlined the foundational propositions and/or tenets of both CRT and CRT/I. To reiterate the combination of the two theoretical ideas, Intersectionality, according to Watkins Liu (2017), adds to CRT assumptions - of which we have previously discussed earlier-(1) intersecting categories (How different social categories interact/co-constitute), (2) multi-level analysis (How multiple levels of society inform and interact impacting lived experiences on social structure and vice versa), (3) power dynamics (Intersectionality examines how power operates in different social contexts) , (4) reflexivity (The constant act of critical selfawareness and reflection), (5)time and space (The inference that social phenomenon and dynamics must be considered within temporal and spatial contexts), and (6) diverse knowledges (The careful consideration of how marginalized groups are positioned within notions of power and knowledge production). Analytically, as a lens, CRT/I:

1. Identifies relevant social categories

2. Pays attention to the social construction of such categories-especially the historical context within which such categories operate

3. Is attentive to how power operates and is challenged

4. Operates at the individual level to investigate how nuanced lived experiences inform social structures and vice versa

5. Pays attention to the treatment of vulnerable voices, perspectives, experiences, and presentations

6. Analyzes the power within and the implications of knowledge production.

Watkins Liu (2017) provides an example how aspects of these frameworks - although not to their fullest extent - integrate. Her study deals with academic scholarship as it pertains to the knowledge production of social movements; particularly, using the American Sociological Association (ASA) and its Collective Behavior and Social Movements (CBSM) while analyzing 10 years of award-winning books-positioned to 
spread knowledge production within the field -that received the Charles Tilly Award for Best Book. CRT/I, as a socio-structural analytic framework, was used to: (1) examine the prominence of knowledge production artifacts via 10 years of Charles Tilly Award for Best Book recipients and (2) to critique social movement studies in sociology by evaluating these books, which are exemplary to the field. The belief of the researcher is that if the intersections of social categories are not Critically structured into forms of knowledge production (the books), it is a disservice to the social phenomena and accuracy of scholarship (Waktins Liu, 2017). The researcher used constant comparative content analysis and coded-by handdata after patterns and themes from the book were identified. Saturation was reached after multiple levels of analysis with particular attention to CRT's notion of whiteness using intersectionality to examine race, class, gender and how these groups were prioritized relative to each other. Additionally, attention was given to citations, the populations that appeared to be marginalized, valued, centered and the overall benefit of what was produced from the study. Next, the researcher investigated and applied the social identities of the authors to the context of the study and finally, came to understand certain power dynamics and exclusions - that may not have otherwise been found-by including lived experiences as a final dimension of the analysis. As a result, the findings revealed that there was a limiting impact of social movements; failing to change the White-male-dominated power structures, which would essentially reinforce the exclusion of underserved populations and the notion of normalizing and prioritizing Whiteness. Additionally, it was discovered that no books with male only authors used ethnography of the $\mathrm{n}=12$ books that used ethnographic methods, which suggests a gender trend within methodological choices. Furthermore, the case studies analyzed in this study primarily privileged White groups and individuals as powerful movement actors, limited Black social movements to integrationist efforts, excluded altogether radical movements such as Black Panthers or the Young Lords, positioned Latin American participants as being permanently helpless while excluding the Brazilian Landless Movement as well. Moreover, $\mathrm{CRT} / \mathrm{I}$ paid close attention to how socio-structural power flows over knowledge production 
through analytic and interpretive frames. For example, this form of inquiry (frame) allowed the researcher to see patterns in voice and who was given "voice" and who was being studied or doing the studying. It was concluded that despite a book's inclusion of underserved populations or discussions of power, the perspectives or voices of such vulnerable groups are not always going to be given priority (Watkins Liu, 2017). To this end, the researcher frames this dilemma in her discontent with how some authors of the texts analyzed, failed to disaggregate the social groups discussed in their texts to fully and accurately account for the realities that may vary among social categories. One example of this is discussed around three countries of women and abortion policies, resulting in the importance of women having control of their livelihoods, health relationships etc. Watkins Liu (2017) argued that by failing to intersect race and gender, the authors who conducted the study framed it as a women's issue, with women as one homogenous group. Nevertheless, excluding the realities of Black and low-income women whose experiences vary.

\section{Data Analysis using CRT and CRT/I in this Study}

For this study, all five of the CRT tenets were used for analysis of the data, which include: Counterstorytelling, The Permanence of Racism, Whiteness as Property, Interest Convergence and Critique of Liberation, to analyze power dynamics, race, and racism in education. For an example, I formulated data collection strategies to capture perceptions and/or responses about the type(s) and/or the pace of institutional change in order to interpret findings by examining the notion of Incremental change for marginalized groups, in that it might be more palatable for those in power. Incremental change is one area of focus under this CRT tenet. Additionally, the CRT/I analytical assumptions I use for analysis were developed by Watkins Liu (2017) and are: a) Identifies relevant social categories, b) pays attention to the construction of such categories-especially the historical context within which such categories operate, c) is attentive to how power operates and is challenged, d) operates at the individual level to investigate how nuanced lived experiences inform social structures and vice versa, e) pays attention to the treatment of 
vulnerable voices, perspectives, experiences, and presentations and f) analyzes the power within and the implications of knowledge production. For example:

a) I identified the social category of each participant (e.g. Race, gender, class etc.) to consider how different social categories interact/co-constitute along with that of peers or colleagues involved in the case or historically.

b) I considered the historical context of race and racism in America, Mizzou and within various social categories over time.

c) I formulated data collection strategies to capture perceptions and/or responses about institutional power dynamics and any challenges for organizational change or institutional reform, to be able to interpret responses based on the connection with this CRT/I assumption.

d) I was reflexive by self-reflecting on why the research was being conducted, for what purpose and how it informs me and ultimately social structures within institutions. I also noted how participants were reflexive in their involvement.

e) I formulated data collection strategies to capture perceptions and/or responses about the treatment of Black students' demands, perspectives, experiences and presentations, to be able to be able to consider social phenomenon and dynamics within temporal and spatial contexts (time and space within the case) to effectively interpret the responses based on the connection to this CRT/I assumption.

f) I formulated data collection strategies to capture perceptions and/or responses about how marginalized groups were/are positioned for notions of power and knowledge production.

\section{Validity of Research}

To ensure accuracy of analyzed and interpreted interview responses from participants and to maintain their real-life story (With thick, rich descriptions) in the data collected, several techniques were 
used to validate findings. First, all participants were given the opportunity to engage in member-checking; a practice that allows participants to read the transcript or report of their interview to confirm the accuracy of researcher interpretations; researcher modifications can be made to the most polished version of the report to better reflect actual meanings (Creswell, 2014). Second, Denzine (1978b:28 in Patton, 2002) proclaimed a principle rule that multiple methods need to be used in every investigation because "no single method ever adequately solves the problem of rival causal factors. Because each method reveals different aspects of empirical reality, multiple methods of observations, must be employed;" this is known as triangulation (p. 247). To further legitimize this claim, Brewer and Hunter (1989:17 in Patton, 2002) stated that the use of multiple methods allows inquiry into a research question with "an arsenal of methods that have nonoverlapping weaknesses in addition to their complementary strengths" (p. 248). Denzine (1978b in Patton, 2002) noted four types of triangulation: (1) data triangulation, the use of a variety of data sources in a study; (2) investigator triangulation, the use of several different researchers or evaluators; (3) theory triangulation, the use of multiple perspectives to interpret a single set of data, and (4) methodological triangulation, the use of multiple methods to study a single problem or program (p. 247). This study primarily use data, theory and methodological triangulation. For data triangulation, interviews were conducted from multiple data sources; including student and faculty perceptions and responses of those involved in the event and/or its aftermath under investigation, to gather similarities, differences, or complexities of the story towards institutional reform. Because this dissertation study is generally framed by Critical Race theory and/ Critical Race Theory and intersectionality to guide analysis and later situated through the lens of the Human Bioecological Systems theory to report findings; thus, it is fitting to offer two perspectives to interpret data as described by theory triangulation. Finally, for methodological triangulation, both interview and document analysis were used as the two methods to study a single case. 


\section{Researcher positionality}

I am Black male of African descent, born and raised in Cleveland/Cleveland Heights, Ohio. As a first-generation college student, I wholeheartedly embraced the act of mentorship upon entering college when it was afforded to me as an Academic STARS student (A summer transition program for recent African-American students entering college). Since then, I continued to perform well academically and became extremely involved in extra-curricular activities, programming, fraternity life (Service and programming), peer mentoring and campus protests for racial equity, safety policies and renovations to communal spaces for Black students. As a result of my undergraduate, graduate and professional experiences at one Kent State University in particular-birthplace of the Black Student Union movementalong with my professional experience at West Virginia University, I have developed in many ways. I have a strong passion for creating spaces, experiences and learning opportunities for people to come to know and embrace themselves, others around them and thrive in spaces that are conducive to their holistic development and spark their concern for: issues of social justice, oppression, equity, and desire for mentorship, to mentor and engage in intergroup dialogues with other racial and social identities to create and learn new meanings and knowledge. It hurts me to my core to watch underrepresented students and Black students like me, be overlooked by their campus faculty and administrators who do not serve them intentionally according to their cultural needs. I bring this with me to this research study as a bias towards my understanding of what happens or does not happen within college universities and how fragmented our university services are across the nation; along with universities who do well with diversity and inclusion practices and foster tenable campus racial climates. However, I have set aside my personal findings and allowed my passion to catapult me into a mindset of curiosity; one that pushes me to uncover the experiences of Black students in demand of change and to understand the ways in which institutional reform happens in the face of demanded interventions put forth by students who feel marginalized. In doing so, I occasionally found it necessary to use bracketing techniques to jot down my own thoughts and 
feelings but stayed un-bias in my data collection and analysis strategies by allowing my report of findings to be reviewed and confirmed by participants to ensure accuracy in my interpretation of their experiences. 


\section{Chapter Four}

This chapter presents the data collected through interviews and document analysis pertaining to events at the University of Missouri-Columbia (Mizzou), which was used as the unit of analysis for this single exceptional case study under investigation. This chapters contains the researcher interpretations of student and faculty responses during semi-structured interviews and documents both during and after the initial 2015 protest incidents to address the following research questions:

1. What are the perceptions of the process of formulating and participating in a curricular response to an exceptional campus racial climate issue at the University of Missouri??

2. What are the perceptions of how this curricular response addressed discontinues in race and racism on campus and contributed to organizational change and institutional reform at the University of Missouri?

To preface the research findings of this case, it is necessary to emphasize the analytical approach to how data was coded to extrapolate meaning from participants of the study. Using the theoretical lens of Critical Race Theory and Intersectionality (CRT/I) together, I also relied upon the Human Ecological Systems Theory to-where possible-situate what was discovered from $\mathrm{CRT} / \mathrm{I}$ within the various systems of the theory. Given how much of what is uncovered from participant experiences by using a CRT/I lens is social interaction, it made sense to help conceptualize them by drawing some correlations to how race, racism and intersectionality impact what happens within the human ecological system on the college campus. Such impacts play a significant role in whether people see the campus in a state of racial unrest, comfortable, or apt to social change. Additionally, I make every attempt to present findings in the most chronological manner possible; however, please note that CRT/I tenants used to code data do not lend themselves to being confined by sequential order, as many of the tenants can and were applied to all themes that will be used to guide the presentation of findings. This overlap in the presentation of data might impact the chronology 
to some extent but it does not detract from the richness and value in the findings themselves. Overall, the research interpretations and meanings applied to data in this chapter's findings are rooted within my philosophical worldview of constructivism and being transformative. Despite my epistemology, it is my hope these findings add to and advance knowledge around curricular reform interventions dealing with race, racism, identity and what lies in and around these ideas influencing organizational change and institutional reform.

\section{Structural Organization of the Chapter}

This chapter will (a) give a brief high-level summary of the case for contextual purposes, (b) introduce participants, (c) introduce documents analyzed, (d) list emergent themes, (e) offer a summary of findings.

\section{High-Level Case Contextual Summary}

This study has been designed to explore the phenomenon of a racially tense campus climate at the University of Missouri-Columbia (Mizzou), a Predominantly White Midwestern Institution. This institution was in the forefront of the media regarding student and athlete protest, leading to the resignation of senior level administrators. The students put forth eight demands to their administrators to address problems felt by African American students. Said demands did not shy away from direct administrative or curricular changes; some of which began almost immediately. In fact, included in this list of demands to their administration was the creation and implementation of a required racial awareness and inclusion curriculum throughout all campus departments and units. Given their problems with race relations and the actions that followed, this case gained national attention. 


\section{Introduction of Participants}

Both faculty and students from the University of Missouri participated in a semi-structured interview to share their overall experiences and perceptions of Mizzou's response to a number of factors related to the case, particularly around the institution's curricular reform efforts following the campus' protest of 2015. A total of 7 interviews were conducted, $\mathrm{N}=3$ faculty and $\mathrm{N}=4$ students. Participants created, taught, facilitated, supported, or enrolled/attended one or both of the curricular interventions that will be presented in the findings.

\section{Faculty Participants}

A. Dr. Stephanie Shonekan served as the Chairperson and an Associate Professor for the Department of Black Studies and Professor of Ethnomusicology in 2015. She was the creator of two curricular interventions: Citizenship @ Mizzou and co-creator of Race and the American story and facilitated/taught both. She currently serves as the Associate Dean for Faculty Development, Graduate Studies and Inclusive Culture in the College of Arts and Science at Mizzou and a Professor of Music and retains a faculty role in the Department of Black Studies. She also serves as the coleader of the Race and the American Story Program, which promotes teacher and student collaboration among multiple institutions of higher education to streamline the program's course and ongoing projects. She identifies as a Black woman of African descent.

B. Dr. Adam Seagrave served as Associate Professor of Political Science and the Kinder Institute of Constitutional Democracy at Mizzou during the 2015 case. He was the co-creator, with Dr. Stephanie Shonekan, of the Race and the American Story Course and taught the course. He currently serves as an Associate professor, an Associate Director of both the School of Civic and Economic Thought and Leadership and the Center for Political Thought and Leadership at Arizona State University. He also serves as the co-leader of the Race and the American Story Program, 
which promotes teacher and student collaboration among multiple institutions of higher education to streamline the program's course and ongoing projects. He identifies as a White man.

C. Dr. Stephen Graves served as a Postdoctoral Fellow in Political Science and Black Studies at Mizzou during the 2015 case. He was a member of the course planning committee to develop the Race and the American Story course and has taught the course. He currently serves as an Assistant Professor in the Department of Black Studies and Director of Undergraduate Studies. He continues as participating faculty for the Race and the American Story Program. He identifies as a Black man.

\section{Student Participants}

A. Jacob Somerscales was a student participant in the Citizenship @ Mizzou diversity training course. He served as a musician, facilitator and provided student input for the training course's design. He has since graduated from Mizzou and identifies as a Pacific Island man.

B. Luke Davis was a student in the Citizenship @ Mizzou diversity training and Race and the American Story. He was a student participant in both and engaged in regularly in course activities. He identifies as a White man.

C. Zoe Romyn was a student participant in the Citizenship @ Mizzou diversity training course. She sat in the front of the course and engaged regularly in course activities. She identifies as a Puerto Rican LGBTQ woman.

D. Ida Campbell-Jones was a student participant in the Race and the American Story course. She engaged regularly in course activities. She was intricately involved in the protest of 2015 as a member of the Concerned Student 1950 group. She is currently a doctoral student at Mizzou and identifies as a Black woman. 


\section{Documents Analyzed}

Table 5

Documents Analyzed

\begin{tabular}{|c|c|}
\hline Type of Document & Name of Document \\
\hline YouTube Video & Loftin Response to Racial Slurs Video \\
\hline News Article & MU Chancellor Loftin announces new diversity initiative \\
\hline News Article & MU Chancellor R. Bowen Loftin announces resignation after backlash \\
\hline Journal Article & The Mizzou Meltdown: How a President Lost Control \\
\hline $\begin{array}{l}\text { College of Arts \& } \\
\text { Sciences Website }\end{array}$ & A\&S Faculty Approve Diversity Course Requirement \\
\hline $\begin{array}{l}\text { Application to } \\
\text { make course } \\
\text { Diversity } \\
\text { Intensive. } \\
\text { Campus website }\end{array}$ & $\begin{array}{l}\text { Frequently Asked Questions: How do I make my existing course diversity } \\
\text { intensive? (Guidelines) }\end{array}$ \\
\hline $\begin{array}{l}\text { Course Evaluation } \\
\text { Summary }\end{array}$ & $\begin{array}{l}\text { Summary of Student evaluations for Race and the American Story, taught by Dr. } \\
\text { Stephen Graves. }\end{array}$ \\
\hline
\end{tabular}

\section{Emergent Themes}

After the coding analysis process was complete, I studied the codes and sub-codes categories to determine the themes that seemed to best reflect the data coded. Despite some overlapping where multiple codes applied to more than one theme, I managed to consolidate codes and sub-codes under what I considered to be a new theme or the more obvious theme. The following themes emerged after the coding process. A general description of the type of content eligible to be categorized under each theme is 
referenced to provide readers with a better organizational perspective of the information that will be presented and where it will be presented.

1. Process of Curricular Reform Implementation

a. Description: This theme will include content related to the process of creating institutional curricular reform efforts following the protests of 2015 at Mizzou. This may include institutional reform efforts made on behalf of senior administrators, faculty/staff, or students and help describe the process used to create curricular responses to campus unrest.

2. Towards Combating Discontinuities of Intergroup Relations in American Society at Mizzou through Education

a. Description: This theme will include content pertaining to Whiteness as Property, the Permanence of Racism, the treatment of vulnerable voices, resistance to reform efforts, how marginalized groups are positioned within notions of power and knowledge productions, counter storytelling, attention to power dynamics, identity development and the curricular reforms or pedagogical strategies used to address structural racism and social interactions in America that help articulate its impact on Mizzou. Perceptions about the most valuable contributions to the course are included here.

3. Required or Optional Racial Awareness Curriculum to Elicit Institutional Reforms

a. Description: Based on the participants values, attitudes, or beliefs, this theme will include content that pertains to student and faculty perceptions on whether race-focused curricular interventions should be required or optional for institutions seeking ways to reform their institutions because of racial campus unrest.

4. Perceptions about the Institutional Response / Curricular Reform Efforts to Change Mizzou's Campus Racial Climate

a. Description: This theme will include content pertaining to the perceived, desired, or actual impact of the institution's response to student demands for institutional reform due to racial campus unrest. This may include data findings for any of the student demands made with special attention to how curricular reforms addressed the discontinuities of race and racism and their contribution toward campus climate change and perhaps what has been or is yet to be reformed.

i. Student perceptions on e.g., the curricular reforms, the institution's response to student demands, teacher preparedness/effectiveness for change, and their/their peer's ability to be more advocacy-driven for social change within the human ecological system.

ii. Faculty perceptions on e.g., course assignments, teacher preparedness/effectiveness on learning outcomes and achievement, their/their students' ability to be more prone to social change of critical importance to note, is that many of the traditional and extended Critical Race Theory and/or Intersectionality tenants of analysis were used to code the data and thus, will be 
interwoven throughout each of the themes to interpret and give Critical meaning and perspective to the findings.

The list of tenants used as codes to analyze the data were:

1. Whiteness as Property

2. The Permanence of Racism

3. Critique of Liberalism

4. Interest Convergence

5. Pays attention to the treatment of vulnerable voices, perspectives, experiences and presentations

6. The role of time and space on social phenomena within temporal and special contexts

7. Identifies and pays attention to the social construction of categories and the historical context within which such categories operate

8. Operates at the individual level to investigate how nuanced lived experiences inform social structures and vice versa

9. Counter storytelling and pays attention to the treatment of vulnerable voices

10. Analyzes the power within and the implications of knowledge production / how marginalized groups were/are positioned for notions of power and knowledge production.

11. Time and Space (The inference that social phenomenon and dynamics must be considered within temporal and spatial contexts)

\section{Thematic Findings}

Before delving into the themes directly, I offer a brief explanation of how my use of an overarching framework, Human Ecological Systems Theory, informed and organized the results of this study. As the people within the human ecological systems at the University of Missouri Columbia-Mizzou continued to experience dissonance and racial campus unrest through their social interactions, it became necessary to address the discontinuities of race and racism and overall oppression felt by students. Consider the various 
ecological systems referenced in chapter one, e.g. Microsystem, Mesosystem, Exosystem, Macrosystem, Techno-subsystem, and Chronosystem. If we conceptualize minoritized students as individuals in the center of the development model and consider the ways in which they experienced their micro, meso and exosystems with their individual identities as factors driving the nature of their social interaction. Particularly, with Mizzou's administration, faculty, classrooms, campus spaces, residence halls; the neighborhood community; friends; members or peers of the Legion of Black Collegians Alliance; the Concerned Student 1950 group; the mass media on the reporting of a controversial murder of an unarmed Black man by police in the neighboring city of Ferguson. Next, we can conceptualize the racial campus unrest over time and the historic influences at Mizzou (Chronosystem) that serve as oppressive legacies, while simultaneously existing to disrupt the current ecological system and create discord in the various social interactions of the individual and their peers, school officials and immediate environments etc. According to the array of racist student experiences in this case, referenced in Chapter One, students felt the need to rally together by creating multiple networks of people, organizations and even the Mizzou athletic department, whereby forcing senior leaders to rethink and address the Macrosystem of Mizzou, which are its norms and values of the campus culture. As Black students involved in this case grew increasingly tired and outraged by their campus racial climate experiences at Mizzou, their demands were aimed at helping to change "the set of social patterns that govern the formation and dissolution of social interactions between individuals, and thus the relationship among ecological systems (Neal and Neal, 2013, p. 729). In other words, those who share the same race, gender etc. [homophily] or commitment to social justice form a set of social patterns that determine how social networks are structured. These social networks influence the kind of social interaction to occur in the ecological environments whereby the focal individual will experience (Neal and Neal, 2013). Accordingly, those who networked around the need for social justice and a more equitable and culturally aware campus constituency pointed to Mizzou's curricular exclusions around culture and inclusivity as opportunities for change. Although, demands for diversity education-generally phrasing-are 
common across higher education institutions, there is a dearth of knowledge around the process of curricular reform and its subsequent implementation. Members of the Mizzou faculty, with varying levels of support from senior leaders, found ways to develop curricula as a response to student demands and to combat the discontinuities of race relations at Mizzou by paying attention to the historical legacies of oppression in American society through a series of educational strategies.

\section{Process of Curricular Reform Implementation}

In February of 2010, White students scattered cotton balls on the lawn of the Gaines/Oldham Black Cultural Center; in August 2014, Michael Brown was shot to death by police in Ferguson, sparking a "Racism Lives Here" rally on September 24, 2014 at Mizzou. Following the shooting death of Michael Brown and just before the start of the fall semester at Mizzou, three queer women (Black, Afro-Puerto Rican, and South Korean) led their peers in the first resistance act; a photoshoot called "Hands up, Don't Shoot," under the name \#MU4MikeBrown, of which they and their peers presented a "Call to Action List" to administrators but it wasn't until the following April of 2015 that university administrators held a town hall to report out on the Progress of the Call to Action list; by then, students' level of frustration grew from a lack of transparency and accountability from leadership. Next, in September of 2015, the Student Government Association President expressed his experience that month with racial and heterosexist slurs, prompting the Chancellor, Dr. R. Bowen Loftin, to state that the comments and incidents of bias are "totally unacceptable." However, students were unsatisfied with the response and protested in late September and early October; just prior to a subsequent racial slur incident where members of the Legion of Black Collegians were targeted during their homecoming court practice on October $4^{\text {th }}$. On October $5^{\text {th }}$, the University's Chancellor, posted a YouTube video to his website in response to racial slurs hurled at students. Critically speaking, he addresses the Permanence of Racism at Mizzou and the mistreatment of vulnerable voices whose racial identity status as Black students made them a target for such demeaning slurs. He laments: 
Its happened again, just last night. On traditions plaza. Hate and racism were alive and well at Mizzou. The LBC Royalty court was in the middle of preparing for their event this week and someone, one of us called them racial slurs and showed how much hate they have in their hearts. Its enough, let's stop this. Let's end hatred and racism at Mizzou, we are part of the same family. You don't hate your family; you don't call your family those kinds of names. Join with me and everyone else who is truly a part of this family and say enough is enough and stop it now.

Chancellor Loftin's plea to cease the racial hatred towards fellow students who should be considered family was not enough to remedy the problem alone. To this end, the "MU Chancellor Loftin announces new diversity initiative" article used for document analysis reported on October 8, 2015 that:

Chancellor R. Bowen Loftin announced a new diversity and inclusion initiative at MU Thursday morning. The initiative is a mandatory diversity and inclusion training program for all faculty, staff and new students entering MU in Jan. 2016. New students who do not complete the training will not be able to register for classes. Loftin said the program is the first step towards a more inclusive campus at MU.

As a senior level administrator, Loftin presented an educational training initiative around diversity and inclusion as a strategy to bring about institutional reform, to combat racial campus unrest issues and work towards improving the dynamics of social interaction within the human ecological system at Mizzou. In fact, Loftin offered his perception of student's ability to change as a result of this educational institutional response over time. Loftin remarked:

There's no simple answer to this kind of issue. Changing people's hearts is very difficult and very challenging," Loftin said. "You do it one step at a time, you do it multiple ways and this is one more piece of what we're doing here at Mizzou to make us a better place. 
Higher Education scholars have presented literature on how colleges change and how institutions are reformed. In some cases, it is episodic, incremental, or continuous. Loftin seems to invoke the tenant of time and space as it pertains to the work necessary to change people's hearts over time at Mizzou, considering the longstanding historical oppressions felt. But Loftin's reaction and institutional response came much too late for students bearing the brunt of a racially and socially tumultuous campus. On October $20^{\text {th }}$, the student group Concerned Student 1950, named in honor of the year the first African American student was admitted to Mizzou, wrote a letter addressed to the University of Missouri outlining historical and recent pervasiveness of racism and discrimination and put forth their list of demands to administration. Nonetheless, after another incident on October $24^{\text {th }}$ where someone used feces to draw a swastika on a residence hall wall, the University of Missouri System president, Tim Wolfe, met with Concerned Student 1950 members privately but did not meet any of their demands. The lack of an action-oriented response led a graduate student to begin a hunger strike on November $3^{\text {rd }}$ demanding the removal of the system president, Tim Wolfe. Joining forces were the Black players-with support from their White teammateswho stood in protest by refusing to play anymore football games until Wolfe was removed from office, which could have cost the university more than a million dollars in forfeitures. Consequently, the UM system president, Tim Wolfe resigned, on November $9^{\text {th }}$ followed by the Mizzou campus' chancellor, R. Bowen Loftin. The November $9^{\text {th }}$ “MU Chancellor R. Bowen Loftin announces resignation after backlash" article used indicated that nine University of Missouri deans called for his resignation and more specifically:

Loftin has been under fire for his handling of graduate student rights and the university's relationship with Planned Parenthood. He was also criticized by students, faculty and staff who said his leadership in the wake of racial tensions on campus was unsatisfactory.

The following was announced by the University of Missouri System (UM system), which is the governing institutional body for Mizzou and other universities under the UM system umbrella, for implementation within the next 90 days to address the racial climate on its four campuses. They are: 
- The creation of a new position for a Chief Diversity, Inclusion and Equity Officer within the UM System

- A review of UM System policies regarding staff and student conduct

- Additional support for students, faculty and staff who have "experienced discrimination and disparate treatment"

- Additional support for the hiring and retention of diverse faculty and staff The UM System also announced the creation of system-wide and campus-based diversity, inclusion and equity task forces, as well as an education training program for holders of the university's top leadership positions. The UM System said it will continue a review of student mental health services and resources.

The remarks of UM System's governing board provided an institutional response; although similar, there are differences from Loftin's prior announcement. Particularly, there was no mention of an actual required or optional curricular reform intervention for students or the broader faculty and staff at Mizzou or within the system. However, their remarks were situated within time and space as they indicated the implementation of reform efforts within 90 days for the entire system in response to student demands. In a 2019 document about the 2015 case analyzed called "The Mizzou Meltdown: How a President Lost Control," Dr. J. Martin Rochester, a faculty member of the system's St. Louis campus, shared how he remembers the university responding to student's curricular demands and said:

On the same day that Professor Click was terminated, Middleton, an African American, received a revised set of demands from Concerned Student 1950 which included: compulsory cultural competency training for all staff, faculty and students, overseen by persons of color; an increase in the percentage of black faculty and staff to 10 percent; and other demands aimed at "advancement of Blacks on campus." The next month, the University of Missouri hired a Chief Diversity Officer with a starting salary of $\$ 235,000$ and instituted a new three 
credit hour "diversity intensive" course required for graduation, focusing on "understanding different social groups..."

Professor Click, referenced in the above narration, was an Assistant Professor of Mass Communications, who joined Concerned Student 1950 in the campus's quadrangle, following the resignations of senior leaders, was caught on camera grabbing the cameras and verbally fighting away student journalist and asking for "some muscle" to remove them; in an attempt to protect the protestors from potential media perspectives counter to CS1950's true story.

Using a Critical lens to more clearly understand what students were demanding, I draw connections to their demand for a curriculum that identifies relevant and intersecting social categories and operates at the individual level to investigate how nuanced lived experiences inform social structures. According to a March 23, 2016 University of Missouri department website announcement under analysis, it was actually the College of Arts and Sciences faculty and not the CDO who set in place a "diversity intensive" course requirement for graduation, of which a version of this idea had been demanded by 200 students in 1990; it was the protest of 2015 that solidified the more than two decades long battle's current relevance that was believed to have prompted its approval on March 14, 2016 but not as the sole direct response. This requirement was a way for the institution to create an academic intervention for more than half of the campus' population that was in peril and gaining negative national attention at the time. This designation of some courses as "Diversity Intensive" would allow Arts and Sciences majors to experience inclusive curricular reform, stemming from the efforts of student activism more than two decades ago. Doing so was clearly a way for Mizzou to embrace the students' historical demand-with a similar intervention-by grounding it in university policy to massify the opportunity for a good portion of students to begin understanding and valuing different social groups other than their own. In fact, the web released document by Yount (2016) frames it as such: 
Students, faculty, and administrators have been calling for curriculum revision to include a diversity requirement for over two decades. In 1990, over 200 students met at Jesse Hall to hold a town hall meeting to discuss racism on campus and a proposal requiring all students to take one multicultural class. Although the proposal was debated for the next decade, no action was taken. Other proposals also fell short, but the events of fall 2015 forcefully demonstrated that the concerns students first raised in 1990 have not disappeared. "It is important to note that this is not something that we whipped up in response to the events of last fall or the student protests," says Elisa Glick, an associate professor of English and women's and gender studies who chaired the Arts and Science Diversity Committee. "The proposal builds on decades of student activism and the work of previous diversity committees. I would be remiss if I didn't acknowledge the work in particular of April Langley and Roger Worthington, whose campus-wide diversity proposal we drew from in crafting our proposal."

Critically, it is important to point out the notion of Whiteness as Property, Permanence in Racism, space and time Interest Convergence at work here. Traditionally marginalized students and presumably some allies, raised their concerns about racism on campus and advocated for a curricular reform, but also that racism could remain pervasive, and without treatment and proposals for interventions to treat the problem were debated, delayed, and left dormant for more than two decades. Space and time are vital constructs for understanding this case, as Mizzou's curricular advancement around the intersections of race and social identities and how inequities exist within these constructs or realities, was stifled for an overwhelmingly long time. Again, we see how the right to determine who, when and how intellectual property can be enjoyed and experienced remained under the control of dominant groups in power, who prioritized Whiteness over multiculturalism in the education system; excluding the identities of thoseunderrepresented-who proclaimed their discontinuities with race relations at Mizzou. This document 
suggests that a lack of treatment to a campus racial climate issue within the human ecological system was evident, as it was blatantly ignored, unprioritized, and placed in the often dogmatic and structurally oppressive institutional structure of an organization. Fundamentally, the delay in action, undervalued the voices of the vulnerable and did not act on an opportunity to improve the treatment of relevant racial and social groups, until it was in the best interest of the university to do so; after being in the spotlight of negative national attention, pegged as a racist campus by its own students. Nonetheless, the academic unit's actions toward liberalism after the added pressure of 2015 protests, included an outline on their department website to detail the "who, what, when and where" of the new requirement, which did not have to overtly discuss race and gender to possess the designation of being "diversity intensive" (DI). In my analysis of the website, their method to assign a course DI status, provided substance and safeguarding to the idea and its implementation process.

To this end, faculty members hoping to receive DI status on one of their courses had to follow a submission process to have their course(s) evaluated and approved by the College of Arts and Sciences Diversity Committee. While the Diversity Intensive Requirement was/is not the primary focus of this study, nor was it referenced in any of the participant interviews, it is germane to the case, as it helps us to understand more about what happened in the area of curricular reform and might prove to be useful to practitioners/educators looking to restructure academic units in this area. Table 6 is the document under analysis, outlining the process:

\section{Table 6}

Diversity Intensive Guidelines

\section{How Can I make my existing course diversity intensive?}

The following guidelines can provide helpful suggestions about how to adapt your course into a diversity intensive one. Importantly, they also provide a list of items to submit for your proposal (Guidelines 2-6 
below). Of course, please contact us at muasdiversity@missouri.edu if you have any questions at all. We welcome the opportunity to help you have a successful DI course proposal.

\section{Table 6 (Continued)}

Guideline 1: Diversity Intensive courses must be designed and taught by MU faculty members for courses in the College of Arts \& Science. Specifically, DI instructors must come from the following categories: tenure track, teaching, NTT, Non-Regular, Visiting, and Post-Doctoral Fellows. Graduate instructors who wish to teach DI courses must meet first meet their department requirements for teaching and then submit signed letter from their Department Chairs, Directors of Graduate Studies, or other departmental approving authority.

Guideline 2: Submit a completed New Course Proposal Form. It will require you to upload and submit the following items in Guidelines 3-6 (below):

Guideline 3: Submit a complete syllabus that includes a "Diversity Intensive" statement (maximum two paragraphs) which explains in detail and describes specifically how your courses meet each of the following two fundamental DI criteria: a) focus on understanding differing social groups (locally, nationally, and/or internationally) and b) exploration of at least one form of social inequality, broadly defined to include class, race, age, ethnicities, disabilities, genders, sexualities, veterans, geographic disparities (e.g. rural and urban), economic and/or resource disparities, indigenous cultures, among others.

Note: Everything you always wanted to know about the Diversity Intensive Statement but hesitated to ask

We understand a short statement cannot possibly convey the richness or complexity of your course. So, it might be helpful to think about this statement as a way to let your students know what is unique about your course and why it qualifies for A\&S DI credit. The Diversity Intensive Statement should be a brief and clearly articulated (200-350 word) description of how assignments, assessment, and other elements of the proposed course demonstrate that the fundamental DI criteria are distributed through the semester rather than concentrated in one area or section of a course. We encourage you to resist revising existing templates from previously approved courses with similar topics or range of material. We each have unique ways of teaching what may seem like the same course. This is especially true of lower division undergraduate courses that require us to cover specific material in a given field of study or discipline (e.g. Principles of Economics, Freshman Composition, Introduction to Religion, etc.).

The Diversity Curriculum Committee may ask instructors to elaborate (as needed) on their assignments or methods of assessment (e.g. rather than observing that a particular assignment asks students to compare statistical data about the rising number of women in biology or STEM, it will be important to provide information about how this "new" information about gender is 
used by students to analyze what this means for the continued gender gap despite the fact that more than half the biology majors are women). Hint: This note is approximately 250 words.

\section{Table 6 (Continued)}

Guideline 4: Submit a completed schedule/calendar or other materials to be covered, with specific titles of articles and studies, and make available copies (links) or related materials as needed by the Diversity Curriculum Committee for a thorough review of the extent to which proposed DI course meets requirements and fully integrates DI criteria above throughout the course. Important: Please indicate whether or not the schedule/calendar is included on the syllabus or as a separate document.

Guideline 5: Grading scale or rubric should demonstrate that diversity intensive assignments constitute a major component of the course grade. Specifically, diversity intensive courses are diversity-centered, rather than diversity-inclusive or enhanced Important: Please indicate whether or not the grading scale or rubric is included on the syllabus or as a separate document.

Optional: While current guidelines do not require sample assignments, instructors have the option of submitting up to three assignments that indicate how their discipline applies elements of diversity to meet both the course objectives for the discipline and the DI criteria for A\&S. Any assignments submitted should be complex enough to engage the student in demonstrable efforts to grapple with complex issues related to issues raised by core objectives related to difference and social inequality, as applied through the lenses of the specific discipline. Neither required discipline specific, nor student learning outcomes for diversity intensive courses should be sacrificed. These optional sample assignments will aid the Diversity Curriculum Committee in considering the extent to which a course, based on its context, scope specific objectives, fully integrates DI criteria.

Approvals: A copy of any service-learning or IRB approvals as needed, as well as any graduate student instructor approvals should be submitted.

Note. Retrieved from https://coas.missouri.edu/diversity-intensive-guidelines

Guideline 3-in Table 6-in particular, is directly in line with CRT/I's analytical lens of identifying relevant social categories, operates at, somewhat of, an individual level to investigate how nuanced lived experiences inform social structures and vice versa and being attentive to how power operates-by exploring inequity. The diversity committee's insistence on faculty having to prove their method of ensuring a) focus on 
understanding differing social groups (locally, nationally, and/or internationally) and b) exploration of at least one form of social inequality, broadly defined to include class, race, age, ethnicities, disabilities, genders, sexualities, veterans, geographic disparities (e.g. rural and urban), economic and/or resource disparities, indigenous cultures, among others, is evidence of my analytical interpretation. While significant and appropriate to require a focus on differing social groups, the framework allows for the likelihood that some faculty might choose to focus on a non-marginalized or non-domestic social group (i.e., international diversity or intellectual diversity), circumventing domestic underrepresented racial/social groups altogether. In doing so, students might find themselves focusing on, for example, people of German decent or conservatives; excluding an opportunity for students to be engaged with coming to understand more about the social groups within their own human ecological systems that are a part of or influence the way they experience campus life at Mizzou. On the other hand, the committee does seek to evaluate and approve proposals that consider power dynamics by calling attention to at least one form of social inequity that exists in a variety of broad categories. The committee further grounds the exploration of social inequities by providing examples of what they are less interested in and how to dive deeper with inequity data found by students to investigate the nuances and how they inform social structures and vice versa. In fact, the committee offers faculty applicants an option to submit assignment examples that show a deep level of engagement. To these end, the document states the following: Any assignments submitted should be complex enough to engage the student in demonstrable efforts to grapple with complex issues related to issues raised by core objectives related to difference and social inequality, as applied through the lenses of the specific discipline. Guideline 5 advances the level or rigor of diversity engagement a proposal course must possess by drawing attention to the word "intensive," clarifying that a DI course is "Diversity-centered" and not "Diversity-inclusive," or "Diversity-enhanced." Doing so, forces faculty to fall from the surface level of the subject matter by creating opportunities for students to engage in a more meaningful and intentional way; a way that brings to the surface robust knowledge, useful enough to highlight the continued impact of 
the inequity and the ways to challenge it for change. The guiding document also applied the lens of time and space to Guideline 4, as the committee sought to evaluate a calendar of assignments, over time, to assess how and when the course will address social phenomenon and their dynamics to ensure frequency of intensive diversity. For the most part, the document concludes that the DI course proposal submission process set forth by the diversity committee, includes aspects of a Critical approach to streamline a formal process of ensuring that courses designated as such are in practice intensified with "diversity." While not full-proof and while some faculty may choose to focus on international diversity or make modifications after approval, the specificity of what DI courses must do and the nature of course assignments faculty must propose they will do, solidifies the likelihood of a course more deserving of the DI designation. Having a somewhat stringent evaluative process might limit the number of faculty desirous of the DI designation but not highly eager to refit or develop a course to reflect the committee's idea of a course that bears the label. On the other hand, what remains unknown for me is if the department/university offered some incentives to faculty to reach a sufficient number of students to increase the likelihood of an effective intervention. Like the incentives offered for faculty and staff to participate in social justice/diversity training.

In addition to the Diversity Intensive requirement, Dr. Rochester also stated in an article that: ...faculty and staff were encouraged to attend social justice/diversity training sessions in order to earn extra points in the university's "Wellness" program.

Given that the Social Justice/diversity training session mentioned in the document-which carries a level of importance for practitioners-was not technically a curricular response to the 2015 case, I will address it in Chapter Five and not in detail here, as it sits outside the bounds of the case. However, Dr. Rochester's reference of the faculty and staff wellness program incentive is consistent with the tenant of Interest Convergence, as faculty and staff might be more likely to participate in a social justice reform effort if they are receiving a benefit of interest, rather than doing it because they see natural value in participating or 
think it is morally necessary. Several interview participants for this study provide us with a first-hand look into the institution's curricular responses and the process of their creation and implementation. Of which may serve to be of great use for higher education practitioners seeking to generate curricula where race and the intersection of identities are at the center.

Dr. Stephanie Shonekan was approached by the university's first temporary leader of a newly created Inclusion, Diversity and Equity division, Chuck Henson, Esq. to inquire about what they could offer as an educational institutional response. Henson was a Professor of Law in Mizzou's Center for the Study of Dispute Resolution and was temporarily at the helm of the new unit until the arrival of the division's first Chief Diversity Officer, Dr. Kevin McDonald. Henson reached out to Dr. Shonekan after hearing her speak from the audience at a campus town hall. To describe what occurred, Shonekan was asked what the impetus was for the curricular reforms that resulted after the meeting with Professor Henson, and if they were a direct response to the protest, she replied:

Yeah, so citizenship at Mizzou was a direct response of what happened in 2015. One of the areas that we quickly realized was missing on our campus was some sort of collective response in the classroom. Right? It's like a pedagogical response. You know, creation of safe spaces is one thing, you know, talking about safe spaces is another thing but there was no response to dealing with behaviors like racist behaviors as we as we had with dealing with behaviors around alcohol or around sexual harassment, or sexual violence. So we had an alcohol.edu training.

Dr. Shonekan's response binds the impetus for the course to time; specifically, as a result of the protest of 2015. She goes on to make clear that talking about safe space is different from the actual creation of safe space; space that is a part of Mizzou's ecological system. A system that encompasses a macrosystem level that determines the culture of an organization's climate, along with subsequent systems that are influenced by the macrosystem; ultimately shaping the nature of social interaction to occur amongst individuals within 
the entire ecological system. To this end, she perceived the reality of Mizzou's need for more than pockets of safe spaces, but rather a need to make the entire campus safe for thoughtful, open, multi-perspective, and respectful engagement. Therefore, a pedagogical response was employed to combat racist behaviors. Racist behaviors that, like alcohol abuse and sexual violence, can be life-threatening and detrimental for the people who experience them. Thus, Shonekan believed that it was the unpleasant experiences and mistreatment of racially diverse students that led to campus unrest, prompting the protest of 2015. Dr. Shonekan went on to describe her observation of required and non-existent offers along with when the idea for a comprehensive curricular intervention was presented:

So we had an alcohol.edu training. We had a training for Title IX, but we did not have anything for, to address issues of racism and other kinds of isms, right, other kinds of discrimination, which I think at the end of the day was what led to the protests in 2015. So, I remember being in town hall meetings as the Chancellor would have, and they'd be, he'd be trying to answer questions and I would, you know, myself or someone else in the in the audience would, you know, say we need some kind of really comprehensive risk response to orient our students to who we are and what our expectations are. So, you know, that was at the time the Chair of Black Studies really worked with supporting the students along with so many others. We have a great faculty and staff at the university. And so when things came to a head and when things were kind of spiraling, you know, in a very complex way, one of the things that came out was the, or that resulted was the creation of a much more structured process of inclusion, diversity and equity.

Dr. Shonekan's mention of what had been excluded or absent from the curriculum is instructive. Whiteness as Property inherently deals with the notion that Whiteness is the standard and thus, those who control it maintains the authority to control and determine who, how and when one can be afforded the right to certain freedoms, dispositions, and enjoyments; including intellectual property. Dr. Shonekan references the 
inclusion of required trainings for alcohol and Tittle IX specifically; a clear indication of what the university deems as necessary subject matter to advance knowledge. While these, two areas are vitally important and should remain required, it was meaningful to the participant that these objectives were given institutional sanction, whereas the treatment and protection of vulnerable voices, perspectives, experiences across race were absent. To delve deeper, in the face of racial and social calamity, it became more apparent that "the classrooms" were exclusionary in their content and required a pedagogical response to the racial unrest, including ongoing content around race, racism and the intersections of social identities. Dr. Shonekan's plea to orient students to understand who Mizzou constituents are and what is expected was a plea for the institution to become concerned with, understand, and commit to the identity development of marginalized populations. Identity, in many ways, is at the crux of the curricular response. An education was required that was concerned with the social construction of identities, the treatment of vulnerable voices and how historical context shapes social interaction and the American society in which we live. Consequently, Dr. Shonekan was given the approval from Professor Henson to begin creating what was first called Diversity@Mizzou and later changed to Citizenship@Mizzou; I will provide my analytical commentary about the rationale for this name change later in this chapter. The following sections explores the implementation of Citizenship@Mizzou as well as a course that followed called Race and the American Story.

\section{The Implementation Process of Citizenship@Mizzou}

Citizenship@Mizzou and Race in the American Story, were administrator-led or faculty-led institutional responses to the protests of 2015. Dr. Shonekan said she collaborated with "some really wonderful faculty and staff" to:

... create a program that would be offered to all incoming students we wanted it to be face to face, not some kind of online program, but a face to face program where we could really like look our new students in the face, you know, ask them, you know, what, what their expectations are of 
Mizzou and for us to be able to also say what our expectations are of them coming onto the campus. We kind of built it around our values. So the values at Mizzou are very clear. They are excellence, responsibility, discovery and respect. And we thought, okay, those four things, if we could really drill those in, we could get to a point where our students understand that you really just can't call anyone the $\mathrm{N}$ word. Because those are those don't reflect the, the values of Mizzou. We also really wanted to be strategic in how we did it, you know.

Shonekan began by designing a training course that would create an opportunity for face-to-face social interaction to occur amid all students; creating an atmosphere of back and forth dialogue through the sharing of ideas and experiences around the institution's values. Using Mizzou's four core values of excellence, responsibility, discovery and respect, to frame expectations, behaviors and attitudes, was a way to bring cultural value and inclusive excellence to the forefront of Mizzou's overall character; directly engaging the cultural values of the macrosystem. In fact, Shonekan expressed that many years of professional teaching experience has taught her that her students do best when they engaged in having conversations with each other. She recommends professors-in some instances-only need to "present the material, step back and moderate a conversation between diverse groups of students, you know, so that they understand that they're all coming from different places, they all have different worldviews." Dr. Shonekan's recommendations are rooted within her experiential knowledge educating students and point to the need for those moderating or engaged in dialogue, to pay attention and listen to the treatment of vulnerable voices and their perspectives, experiences and presentations that possess the potential to advance ones' knowledge of both their own and their peer's racial and social identities. Such networked communication among multiple identities from the onset of ones' collegiate journey, sought to set the tone and temperature for social interaction and comfort within the institution's microsystem. During the process of implementation, Professor Shonekan listed other strategies and concerns that led to the development of Citizenship@Mizzou, as she recounted: 
And but then there's this material that you're all trying to, to, to address. So I decided to bring students in, in with me to the drawing board. And because I studied music, I wanted music to be a very central part of the program. So, really Citizenship@Mizzou was a direct response to what happened in 2015 . I wanted to make sure, also knowing how much work those students had put into the campus already, that we valued, we showed that their contributions would be valued, so I made sure they were paid. I wasn't going to have them up on any anybody's stage without some kind of renumeration. You know, if the university says it's about its values, student input, and student engagement, then this was a really great, great way or opportunity to show.

The professor recounts of her use of Music as another glimpse into the creative nature of pedagogical practices employed to ground the course along with Critical face-to-face dialogue. The professor's insistence to both include students in the decision-making process and to ensure student participants of the newly implemented course were paid, suggests a real concern with how such vulnerable populations of students or advocates were being treated on campus, not being used for free labor, and modeling respectable behaviors. She was careful to respect their perspectives, used to drive course content and their experiences and presentations during each course session, to emphasize the institution's value in their contributions. She recalls their hard work and contributions during the protests to fight for such curricular reform and vowed to make sure their experiential contributions to advance knowledge was being valued by framing it as the institution's economic investment into the reframing and resocialization of its campus climate. While not overwhelmingly significant in monetary value, she felt it was a necessary step for the institution to put its money where its vocalized support is to begin shifting how marginalized populations see themselves positioned within notions of power and knowledge production, when being educators to their peers is not their primary focus. One student, Jacob Somerscales, when asked to 
describe the process of creating and implementing the training, described his perceptions about the initial stages of his involvement and support of Dr. Shonekan's efforts from the start:

Yeah, so you know, obviously my position was a, quite a bit down the ladder. I think. I don't know much of what went on between administrators and Dr. Shonekan. But I had been a former student, and, you know, just a friend of Dr. Shonekan for a while. And you know, having taken some of her music history classes and musicology classes. So, she kind of just came to me with basically, I guess, the idea, the sketch for Ctizenship@Mizzou and we knew that we wanted it to be focused on or at least centered around music. Because, you know, this is a kind of a popular culture, consumer social circle, you know, a university, I mean, everyone knows popular songs and stuff like that. So, we wanted to try and create a message that would sort of meet people where they are. I guess the other option would have been, you know, make it a very textbook kind of teaching process, which most kids don't really receive well. So yeah, so I mean, at that point, we had kind of come up with just the basic blueprint. And then I, you know, I had been playing music and in bands and backing up vocalist and stuff for a while in Colombia and so I kind of assembled the avengers, people that I knew were good musicians but more importantly good people who I knew shared the value of, of kind of respect and these, these four pillars of Mizzou... The four pillars of Mizzou are respect, responsibility, discovery and the other one that I always forgot [Pause to think] excellence. And so those are kind of like, ingrained into what you know Mizzou always promotes itself as being. So the idea was to kind of live out those values...

Jacob's role as a student was not networked directly with Dr. Shonekan and the administration's network to prescribe a curricular plan to influence the focal individuals within the institution's human ecological system. Much like Bronfenbrenner's theory, the focal individual(s) of the system may not always be 
involved at every level of the system in order to be directly impacted by the decisions of those in power who seek to define the system at, for example, the macrosystem level. I do, however, assess that Jacob minimized and undervalued his contributions to the implementation process, as his student perspectives networked with Dr. Shonekan and helped shape the nature of the instructional strategies and musical atmosphere employed for learning. His "boots on the ground" approach helped to drive critical components of the course; namely, music and who and what value each presenter should represent. Being, both, a Pacific Islander and a formally educated musician, speaks to his place within the institution's underrepresented racial and academic groups. Thus, a Critical lens forces us to see how Jacob was situated within notions of power to be a part of the process to advance knowledge in predominantly White spaces. His racial and social identities, at the individual level, allowed for an investigation of both his and his musician peers' nuanced lived experiences and how they might influence social structures and vice versa. For each session, music was a way to-first-centralize and name a common interest amongst all identities present, followed by the sharing of four diverse individuals' counter-stories linked to Mizzou's core values around the notion of good citizenship. Upon learning of the centrality of music, I immediately wondered or was a bit troubled that the central focus was not racism and the intersections of race; later, I came to understand their rationale to take a careful approach to addressing the campus racial climate issues given the varying backgrounds and perhaps, unidentified identities of the audience being served and the limited time for each session to do an effective deep dive into the material. This is reminiscent of change happening at a slower pace, but it was the first curricular response to the Concerned Student 1950 group's demands, to broadly ensure all future Mizzou students had a foundational starting place to chart the journey towards racial/social awareness and inclusion.

While not mandatory for all students, faculty and staff as Concerned Student 1950 wanted in their letter of demands, the training was mandatory for all freshmen and transfer students slated to attend Mizzou. Dr. Shonekan described the thought progression of the mandate, stating: 
...it was mandatory and we had to really, you know, put our foot down and say look, if you come, if you decide to come to Mizzou, here's what we need you to do.You have to take this two hour session, sometimes one and a half hours depending on how many students we had in the auditorium. And to be able to register for next semester, for your classes the following semester, you would have to go through Citizenship@Mizzou.

Additionally, there was a firm stance to require this training course for new freshmen and transfer students, which make participation in a session important and necessary for Mizzou students clear from the onset of enrollment. After the training course's first iteration, students who completed the training provided mainly positive verbal feedback, which led to its renaming and a spinoff course to follow.

Diversity@Mizzou was the first name assigned to the training course when it was initially implemented. After a Black student approached Dr. Shonekan, who took issue with the general use of the word "Diversity" and offered the name Citizenship@Mizzou because they were all citizens at Mizzou. Professor Shonekan describes this reality within the confines of space and racial or social backgrounds that drive emotions and attitudes around the idea of diversity. She explained:

You know there's some students who just don't like the word diversity. Particularly living in a state like Missouri, you know, there are some, there are some trigger words, you know that that trigger people off depending on where you stand in the in the sort of identity spectrum, right. And first, for some people in Missouri, you know, small rural towns, you know, or even some suburbs, you know that word diversity comes with a lot of baggage. You know, which I don't think is bad, but if we're really trying to reach a certain group of students, we need to figure out, figure a way around it. So I remember it was one of the students who had some issues with the title diversity at Mizzou.

A Critical analysis of this name change suggests the influence of a so-called colorblind philosophy that takes away the backdrop of "diversity" or "difference" which hint strongly to the nature of the training's 
sole purpose, to discuss the multiplicity of identities at Mizzou. While it is true that all the students would have a common bond of being "citizens," adopting the umbrella identity of "citizenship" alone moves further away from the inclusion of race, racism and the intersection of social identities that might be gleaned from the tittle alone. According to DeCuir and Dixon (2004) doing so is consistent with CRT's tenant of Critique of Liberalism that perpetuates colorblindness "as a way to justify ignoring and dismantling race-based policies that were designed to address societal inequity." Adopting such ideology does not eliminate the likelihood that acts of racism will persist. To further expound this analysis of Mizzou's implementation process, DeCuir and Dixon (2004) offer the following:

Under the notion of incremental change, gains for marginalized groups must come at a slow pace that is palatable for those in power. In this discourse, equality, rather than equity is sought. In seeking equality rather than equity, the processes, structures, and ideologies that justify inequity are not addressed and dismantled. Remedies based on equality assume that citizens have the same opportunities and experiences. Race, and experiences based on race are not equal, thus, the experiences that people of color have with respect to race and racism create an unequal situation. Equity, however, recognizes that the playing field is unequal and attempts to address the inequality. Hence, incremental change appears to benefit those who are not directly adversely affected by social, economic, and educational inequity that come as a result of racism and racist practices (p. 5).

To be clear, Dr. Shoenkan's agreement to change the name of the training course is not an indication of her having a desire or plan to exclude race and racism to push a colorblind ideological agenda. However, her assessment of the use of the word "diversity" carrying baggage and being a trigger word for some people is what drove the decision, as a way to temper students' initial emotions and attitudes towards participation upon learning they would be required to take a diversity training course. In my analysis of this section of data, I do not attempt to discredit the great work of Dr. Shonekan but draw attention to my 
critique of the liberalistic efforts of the institution's senior leadership that placed such a heavy responsibility on her to execute such critical and massive work, primarily alone and in addition to existing roles at the university. DeCuir and Dixon's (2004) work guided my analysis decision as they described a similar circumstance around curricular reform implementation, as one high school district hired one African American to implement multicultural courses and explore how they changed racist practices and policies. Here is DeCuir and Dixon's (2004) example of a CRT analysis under Critique of Liberalism:

A limitation of the liberal commitment to diversity was manifested in Well's hiring one person, an African American, to attend to the school's diversity initiative. Making her responsible for teaching all the multicultural courses and providing all the programming and professional development in the areas of cultural sensitivity and awareness demonstrates the school's lack of commitment to diversity. This token commitment to diversity, which rested solely with one person, and encompassed a wide range of responsibilities, essentially ensured that change at Wells would not be sweeping or immediate. Thus, with the limited human resources Wells employed to "diversify" the school and the curriculum to create a more diverse and inclusive schooling environment, it guaranteed that changing the racist remnants of the "Old South" would not likely happen quickly, but incrementally and superficially instead, if at all (p.5).

Given the increase in national attention to this case, one might assume that an abundance of human resources, not to mention financial resources, would be directed towards a curricular reform intervention plan. Granted, she did not complain or hint about her increased responsibility in any way and gave credit to Professor Henson for his support of her, I did not gather from my time with Dr. Shonekan, that a team of faculty and staff were tapped and secured by senior leadership but rather her own professional networks of people she consulted to garner support for Citizenship@Mizzou. While Dr. Shonekan appeared to be innately driven and genuinely passionate about carrying out the work of inclusive excellence and educating 
students to improve intergroup relations, some students who admire her greatly, were concerned for her bearing the brunt of this work alone. One student, Ida Campbell-Jones, stated there was much unnecessary pressure placed on the faculty teaching RAS, because there was not much support from faculty outside of the departments represented by the planning faculty.

Such overtaxing of race-focused work with limited human and financial resources can certainly lead to Racial Battle Fatigue. Racial battle fatigue is a theoretical framework that describes the racial weight Black/people of color face as a result of systematically inequitable structures that perpetuate notions of institutional racism. It is the Psychosocial, psychological and physiological stress response associated with being Black/a person of color (faculty, staff or student) in predominantly White spaces. For example, racial battle fatigue signs may include frustration, anger, exhaustion, social withdrawal, depression, anxiety, stereotype-threat, physical burnout (e.g. insomnia, head/backaches, high-blood pressure) (Smith, Allen, \& Danley, 2007). Faculty and students engaged in this work on an ongoing basis are likely to become overwhelmed; faculty especially, carrying the burden of trying to cater to people must be concerned with the delivery of their curricular offerings and approaches to avoid alienation and a disinterest in subject matter. One of the precautions Dr. Shonekan describes is around how explicit she and her team decided to be with students about the historical nature of racial campus unrest and how power was being challenged during the protest of 2015 , leading to the creation of the course.

Similarly to the dynamics of the name change issue, when asked whether race and racism or identity were outwardly spoken about to describe the course to students, Shonekan shares her perceptions of language use and words that guided her first iteration of the training course, stating: You know, Bruce, I think there are some, some words that have now entered the register of diversity training, right? That a very good word, microaggression, implicit bias, white, white privilege, you know, these are terms that have now entered the lexicon that are very important. But, bringing them into a training for kids who have just come out of high 
school, you know, that don't think that there's anything wrong with the way they've been brought up, you just have to sort of walk that through that line carefully. That's my own personal view. I mean, somebody else would, you know, come with a different approach, but that was my, my approach.

The professor describes her careful nature of directly addressing the aforementioned terms that have entered the so-called register or lexicon of diversity training terms to avoid too much push-back from those who are from racial/social groups that have not had the opportunity to explore the intersections of identity groups or question their own upbringing, values, attitudes and belief systems. It appears that the mere mention of coined diversity terms and ideologies - for this course in particular-might present some resistance and/or apathy towards the content of the training or that could not be adequately addressed in the time allotted and in such a large setting. However, as another indication of how time and space plays a role in institutional response to racial climate issues, these attempt to be more indirect with diversity terms and the campus unrest incidents of 2015, through the use of the institution's core values, were a significant distance in time and space from when students wanted a more direct approach and desired to gain a deep understanding of the protests of 2015 that gained national attention. In fact, Dr. Shonekan explained that:

And we were there were some people, some of my colleagues, the faculty members up on stage who were like, maybe we shouldn't talk too much about "Concern Student", you know, let's just keep moving and so on. I know there is a hesitation with talking about history, even the most recent history, right? And so, we kind of tried to do that and then we would always get questions from the students from the participants in the room in the auditorium. You know, so can you tell us what, what happened last semester or last year? A Critical lens focuses attention to the construction of relevant social categories, especially the historical context within which such categories operate and to be attentive to how power operates and is 
challenged. Thus, for reasons-arguably-valid, the initial construction of the training course intentionally opted not to highlight the events of 2015 in any literature about the required training or during the session, to limit potential resistance and disengagement from the participants. However, after this experience, Dr. Shonekan modified her approach slightly stating:

Every time after that we would, we would definitely say this was this is something we created because students really raised the question of who we are and what were we about, you know, so we're going to take time, these two hours tomorrow. Talk about that, you know, and I did, I did. I knew all the students personally who had done the work, they were exhausted, they had done so much already. It was time for them to really get back to what they were here for, you know, and I didn't want to burden them with, you know, coming into the room. I think I did [Call upon one of the members of CS1950] one time when one of our students [A regular student facilitator] was off. One of the Concerns Students was a musician [in the session]. So I asked him to come in and, and uncover and he did and he was great, you know, but for the most part, the students knew what we were doing. I didn't like write up a thing and says hey, there was [a group called] Concerned Student $1950 \ldots$ This is one thing that was not my job, that I felt that that was either the ID [Inclusion, Diversity and Equity] office or the administration should be able to count this towards what they were doing. I wasn't going to be the one to write that up or make a statement about that. We were just about doing the work.

Dr. Shonekan's shift in curricular messaging came as a result of her reflexivity an act of Critical selfawareness and reflection (Watkins Liu, 2017). She used the experiential nature of social interaction she and her colleagues had with students initially and made adjustments to the pedagogical framing of the session, rather than blame or ignore the voices of the students; a clear display of an equity-minded approach to yield more successful learning outcomes. The decision to both not burden or rely upon the 
experiential knowledge of members of Concerned Student 1950 is in line with Shonekan's insistence on an attentiveness to how vulnerable groups have been/or were being treated and operating at the individual level to investigate how, for example, the nuanced lived experiences of the Concerned Student 1950 member(s) felt about sharing their counter stories of protest participation and campus activism. The ascribed analytical framework reminds us to consider how traditionally marginalized people are positioned within notions of power and knowledge production; not all will feel it is their place or possess the same level of comfort with sharing their experiences and may deal with racial battle fatigue. Thus, peers or institution officials should not rely solely upon them, overburden, expect or wait for them to educate their peers in the courses where-they themselves-should have a right to be present, listen and learn as those from dominant groups.

Jacob Somerscales, to a degree, validates the above synopsis of students' experience with racial battle fatigue as he recounts his perceptions of some Black students' reaction to the training course's subject matter. He recounts that:

...it was always kind of those, you know, you notice that a lot of the black students would be there and they kind of, you know, this is something, you get the sense based on their body language that this is something that, you know, they think about a lot. And, you know, it's something that I guess they, you know, to varying degrees have had to, you know, just be conscious of in their life, whereas a lot of the kids that were like, you know, the kind of stereotypical, like, frat guys were, you know, widely the least engaging and also the least, likely to kind of like turn around most of the people that we turn around, we're like white women.

Jacob's perceptions, hint at the way some vulnerable students respond to being situated within notions of power and their oppression in an academic setting with their White counterparts. A setting in which, some of their White peers treated the content discussed, that disproportionately disenfranchises Black people, 
with a lack of engagement and display of their desire to be enlightened/more equity-minded.

Nevertheless, Jacob perceived a lot of the Black student's body language to have resonated with the material given their own lived experiences on a daily basis; which for some, can display as being frustrated, angry, hopeless about their ecological system's ability to change and tired of having to think about the course topics so much. For others, it may display as highly engaged in the mission to share their experiences and remain motivated to seize all opportunities to advance knowledge around their day to day struggles in predominantly White spaces on campus. As noted above, Jacob perceives the White male students - primarily resembling the oppressive mindsets of historically White fraternity members - as being least engaged and willing to "turn around." Their apathy toward social justice and embracing Mizzou's values for good citizenship hints at the notion of a permanence of racism and a need for identity development, given the lack of interest in the treatment of vulnerable groups different than their own. While this section of the chapter is primarily dedicated to the perceptions of the process of implementation for the curricular interventions, it is important for us to consider the perceptions of student responses to this intervention, as it is, in my opinion, a necessary part to consider when attempting to implement such a reform effort. Student perceptions are valuable in their ability to inform practitioners/educators of what to be prepared for and how to create an atmosphere reminiscent of the transformational change sought after to achieve, which is the central to the purpose of Critical research and practice. Therefore, when asked if the session reached the demographic of students who needed it most, Jacob shared his perceptions of his peers reaction and the influential social networks shaping their attitudes, beliefs and behaviors within the microsystems, by confidently stating:

I think all of the people, if I recall correctly, who were talking about themselves coming into this having a certain preconception, and us turning them around, were more women. So, yeah, it doesn't come as much of a surprise to me because the kind of White fraternity culture is, much less inclusive. They're, almost archaic in their inclusion kind of methods 
and they're just a little bit either ignorant or, willfully or not, or just, unlearned on the topics. So yeah, I mean, I definitely felt like they needed [The training] the most. Again, Jacob calls out the permanence of racism and Whiteness as property given the stability of the historical "archaic" and inequitable structuring of historically White fraternities. This is one of the networked relationships within the human ecological system that directly impacts the focal individuals; in this case, it is presumably some of the White males who hold membership in these organizations. This is not generalizing of all White males in each session as being fraternity members but much rather a recognizable portion to reach his assessment. Under analysis, the idea in the example he provided forces us to consider the impact of the historical legacies of structural oppression on marginalized groups; their influence to afford people in positions of power a right to hold certain dispositions as underscored by Whiteness as Property.

Overall, although it is my assessment from the data, that more senior level resources infused into Dr. Shonekan's desire for a comprehensive curricular program would have improved her mission, the university was very supportive of her efforts towards rebuilding a better campus racial climate and allowed for her to have a smooth implementation plan without bureaucratic constraints. After the implementation and rollout of Citizenship@Mizzou was underway, it paved the way for the creation of a second curricular reform with a course called Race and the American Story.

\section{The Implementation Process of Race and the American Story}

When asked what led to the creation of a course situated around race, Dr. Adam Seagrave reflects upon the role of Citizenship@Mizzou in the development of one called Race and the American Story(RAS). He shared:

...the course itself was built on the already existing orientation program that Dr. Stephanie Shonekan had developed the year before. So, so she had developed this orientation program that was required of all incoming Mizzou students. It was...I think, a two hour or 
so, in person, you know, event essentially that involves music and some talks and conversation that all students were required to, to complete as they entered Mizzou with the goal of healing the fracturing on campus following the Michael Brown killing and the protests and incidents that you mentioned, right, the many racist incidents on campus that were just surfacing at that time and so, the university was trying to heal that fracturing on campus first through this orientation program that Dr. Shonekan designed and launched, and it was successful, but she received feedback from this. A lot of the students who participated in this in this program, they you know, it was good, but it just wasn't enough. Like it was too short. It was a one-time thing. You know, they needed more...

Seagrave's perceptions of what led to RAS, stems from the initial institutional curricular response to student demands with Citizenship@Mizzou, which he describes as fulfilling a need for healing social networks after much racial unrest. He situates the impetus for both reform efforts within the notion of time and space, of which he perceives to have stimulated the need for healing after the death of an unarmed Black man by police in a neighboring city. The racial tensions felt by communities, both nearby and across the nation, fueled the discontinuities among racial groups within Mizzou's ecological systems, resulting in several racist incidents of which Black and other underrepresented racial and social groups became victims. Dr. Seagrave's perspective is that students perceived the initial curricular institutional response to be good, but not enough; leaving them wanting for more. To some degree, I interpret the broad curricular approach and limited training time of Citizenship@Mizzou align with Critique on Liberalism. Whereby, change happens at a slow pace and the concern for how people might resist it, supersedes the inequitable treatment of underrepresented groups pushing for change. To be fair, we should also consider time as a driving variable to this broad approach, in that it was important that the university responded to student demands for a curricular intervention with swift action to begin remedying the fracturing that occurred over time. In this case, the university made a quick effort to implement an intervention that could be instituted without much difficulty or delay to start the path 
toward healing. And from it, students desired a deeper dive into the discontinuities in racism, race and the intersections of race in American society. From said requests by students, Dr. Seagrave explained his approach to create an additional curricular response, saying:

And, and so actually at that point, so I had been interacting with Dr. Shonekan and you know, various meetings and things like that on campus since I arrived at Mizzou. And I had had the idea to, to do something on my end to try and to try and undo the fracturing, bring students together, promote learning and understanding and, and those things. So, we got together the two of us Dr. Shonekan and I, I kind of pitched to her the idea of a course like a semester long course. And then she immediately said, you know, that's that's exactly what I've been thinking because the students have been coming to me saying that we, we like, what, what the orientation program is doing, but we need more, you know, we needed to kind of extend across a whole semester. So Both of our, our thinking just aligned right there. So it was, you know, it was in response to student demands for more of what Dr. Shonekan was providing, and you know, more learning, more awareness. And and so and then it was a response to students and then also a response to the faculty, she and I kind of seen what was going on on campus and thinking, you know, we've got to do more. And so let's design this semester long course, to try to try to meet that need.

Under analysis, the collaborative work of two faculty members aligns with the treatment of vulnerable individual's voices, perspectives, experiences and presentations. Both Dr. Shonekan and Dr. Seagrave were determined to respond to Black students and some faculty demands and desires for a deeper dive into a curriculum around the discontinuities in race, as a strategy to reconcile social relations, that were fractured by racist rhetoric, behaviors and attitudes. Dr. Shonekan recalled that some students would comment that two hours was too long but others approached her wanting to know how they can learn more about "this," "what's next," because they felt they were only "scratching the surface." She and her colleagues would direct them to 
take courses in Black Studies, Political Science or History at the time. Present here is the professors' internalized concern and inclination to propose transformational curricular efforts to fulfill the need for more awareness and understanding of identities within Mizzou's human ecological system. Dr. Shonekan details her and Dr. Seagrave's interactions as they approached the idea of implementing a new course as:

Adam approached me and said, you, you know citizenship. C@Mizzou is great, but how about we created something that that gave students an opportunity to really dive into the history of the ways in which race has been... the discourses on race have evolved. And so I thought that was a an excellent idea...so together we designed Race and the American story as a one credit course. And we just said, you know what, let's just see, you know, let's just enjoy not mandatory, it's just a one credit course students can take it from any discipline. And let's see if we can ask students to read these essays, these poems or speeches, all these primary source documents that come straight from the Declaration of Independence all the way to Obama's race speech. And I included, sometimes I'd include Trump's op ed on the Central Park five that he had written some years ago because you know, I think it's all there you know, we can trace it all the way to that.

First, the mere need to expand upon the institution's initial response to student demands with a formal one credit hour (later 3 credits) course, tells us that both students and some faculty did not perceive the initial intervention to be sufficient for fully addressing Mizzou's campus racial climate issue. Therefore, a racefocused course, situated within a historical context, was proposed to speak more directly to the structural implications of race in American society. The name of the course includes the word "race," keeping an emphasis on race/identity being at the center. Next, the faculty sought to identify relevant social categories to explore the historical legacies of race in American society by examining historical and foundational documents from a Critical perspective to prompt dialogue. This curricular strategy would afford students an opportunity to investigate at the individual level, the ways in which social structures have informed the lived experiences 
of different social groups and vice versa. While I see the proposal of an additional and formalized curricular intervention as a step towards institutional transformation, I wanted to know if this idea was supported by senior university leaders and considered an "institutional response" to the protests of 2015 and, if so, how widely this course was touted as such. Both Drs. Shonekan and Seagrave described Race and the American Story as more of a student and faculty-led response than an "institutional" one, given its grassroots implementation. Dr. Shonekan shared that she and Dr. Seagrave called upon several of their colleagues to join a course planning team to detail the course syllabus and determine how the course would be experiences by its attendees. The interdisciplinary approach included faculty from, Black Studies, Political Science, the Kinder Institute and History. Dr. Shonekan shared the following:

We, the four or five of us, got support from our units, and the departments, so Kinder, Black Studies mostly and then tangentially I would say political science and history. To create it, we just wanted to see... it was very much experimental you know, we wanted to see if, if this would work, if there was a thirst among the students for this kind of experience. I would not call that one an institutional intervention, I think, Citizenship@Mizzou was but recently American story was an intervention that some faculty created with the support of their particular units.

Dr. Seagrave shared similar perceptions of RAS's label not initially being an institutional response:

...we ended up working with the administration. And, you know, they knew what we were doing, and they approved of it and supported us. But, but it wasn't as much an institutional initiative as it was a faculty and student initiative from the beginning. I mean, I think I think now, it's pretty, you know, it's pretty widely known. I mean, we, we were interviewed a number of times through the campus media and local media, you know, soon after we started the course. And so we got the word out there. There were five sections of the course taught 
in his first semester, and they've continued to really ramp it up at Mizzou. It's [now] become a required course for a new residential college program that they've got their at Mizzou. So, so it's become institutionally embedded now, but it wasn't initially an institutional response, its more of a faculty student response with the support of the institution, if that makes sense.

While the creators of this course did not report RAS as being an institutional response in the same way Citizenship@Mizzou was, I gently push back on this idea in a semantical manner, as I interpret the faculty to be representatives of the institution who initiated the reform effort. Therefore, I have framed the implementation of RAS as a "faculty-led" institutional response and Citizenship@Mizzou as an "administrative and faculty-led" institutional response. Whether or not direction from a senior level administrator prompted the change or impelled the faculty to design a new course, the students' experience and engagement with the course would not differ. Additionally, given the faculty's influence and control within academic affairs, they held institutional power to shape and determine the institutions' curricular agenda. Furthermore, even if the senior level administrators prompted an idea to implement an additional curricular reform, they would have consulted faculty to lead the effort. I felt it was necessary to provide clarity around the co-creators language used to describe the type of response RAS was because of this study's focus on the curricular "institutional responses" following the case in 2015. Despite the course being the idea of senior level administrators or junior and senior faculty, the course is an outgrowth of the 2015 case and the subsequent demands, strategies and efforts to reform the campus climate through a structured curricular intervention designed by officials of the institution. Moreover, Dr. Seagrave's latter perception above describes RAS as being "institutionally embedded now" given its continued status and being a required course for the Kinder Institute's residential learning community. The Kinder Institute on Constitutional Democracy is an interdisciplinary academic center at Mizzou with expertise in research, teaching, and community engagement and is guided primarily by faculty from Political Science and History, who study ideas of American political thought through the examination of the events of American founding and their continued impact on society 
today. It was appear that the institution's value in Race in the American Story was deemed worthy enough to become a part of the Kinder Institute Residential Learning Community required course curricula.

The following section offers insights on the details the faculty committee experience designing the course to shed light on the perceptions of the implementation process. Dr. Shonekan described it as such:

...we [Adam Seagrave] just sat down over lunch, several lunches and coffees and we designed an outline of the kinds of readings that we would want to have in that course. Thought about what it would look like for one credit, so [we] knew about the number of hours...we didn't want to call it a one credit, but make it a three credit you know, either for ourselves because it was an overload and for the students. We didn't want to also push students out, you know, and so there were many reasons why we wanted to make it a one credit. And then we, I think we did, we did propose it to IDE (Inclusion, Diversity and Equity office) I think and IDE was very very receptive to, I'm glad I just thought of that, to helping to support the dinners. So we did get some money from IDE to pay for the dinners. I believe, if I remember correctly, I have to ask Adam, to pay for the dinners with, that brought us all together. And then we were also planning a big event to bring in a big speaker to talk to the students. And we were, I think, in talks with IDE about support for that.

Analytically, I bring attention to Dr. Shonekan's identity as a Black woman to shed light on how she is positioned within notions of power to advance knowledge. Despite her collaborative efforts with a non-Black faculty person, she was able to maintain a position of power and allowed her voice, perspectives and experiences to be heard as a main factor during the implementation process of RAS without being silenced by her administrative superiors. On the contrary, she reported that the IDE Administrators were very supportive and giving of financial resources to fund her and Dr. Seagrave's efforts to hold out of class events. In addition to administrative support, they received support from their colleagues who joined the team and contributed 
significantly to the completion of the course syllabus and pedagogical practices employed to advance knowledge. Both Professor Shonekan and Dr. Seagrave walk us through the next steps as Shonekan explained:

After we had an outline, we, Adam and I met with Justin Dyer, who is in charge of the Kindle Institute, we had a meeting with Justin, April Langley, who was a faculty member and in Black studies, she's now the chair of Black Studies. And Steven Graves who is also a faculty member in Black Studies. So, there was five of us that just sat down and looked over the draft [Syllabus]. April did a really great job, she's a literary scholar. She did a great job of making sure we had some Phyllis Wheatly, some Francis Harper, some kind of Julia Cooper in the syllabus, you know, Ida B. Wells, we had some women but we didn't have enough, you know, so April did a good job of bringing in some Zora Neale Hurston and then we kind of just talked through it, you know. As an ethnomusicologist, I included an assignment where students would have to create a playlist, you know, to bring or to kind of lighten it up, you know, I think music can help lighten things up, but have some deep ramifications, you know, so yeah, you know, just throw in a public enemy song, you know, flavor flave and his clock... "oh, what are they talking about?" [People might think] Yeah. So we collaborated on the syllabus that we ended up using and we're still using that syllabus for the most part. As I said with making it a three-credit course [After the first iteration of the course] we were able to add some components, add some assignments, add a book, you know, in addition to all the readings on Moodle [Online learning platform], had more hours for deliberation and reflection.

The committee's approach suggest a commitment to intentionality with their selection process of authors and scholars to include in the curricular plan. To this end, the committee was concerned with their treatment as "historical vulnerable voices," who-even in death for some-hold perspectives, experiences and presentations vital to the creation of new knowledge, awareness and understanding through their contributions to the world of knowledge. There was a clear concern for how the historical activists and literary 
scholars, named above, were positioned-in the past and present-in position of power to advance knowledge around race and racism in American Society. The faculty planning team considered the relevant social construction of identities (e.g. Black women) that were originally not sufficiently included in the course syllabus but were added to ensure opportunities for students to explore how intersecting identities influence structures and vice versa. Dr. Seagrave validates Professor Shonekan's account of implementation with a few more important details of the process, as he recalled:

And so the five of us I think it was five of us then sat down and designed the syllabus over the course of a few weeks or something going back and forth and soon after the course launched, we started meeting as well with the, I believe his title was Vice Provost for Inclusion, Diversity and Equity at Mizzou. Started meeting with him to talk about, you know, the sort of institutional support, help, you know, co-sponsoring lectures and making him aware of what we were doing and, and that sort of thing. So, so it was it was basically a planning team of faculty members. And then also in discussion with university administrators who, who were interested in what we were doing. Yeah, and then of course, you know, with the student feedback, I mean, that was a key part of it from the beginning too I mean, we were always listening to what our students were saying in our other courses and in the Race and the American Story course, and we would consult them, you know, and ask them what they thought and get feedback and then incorporate that into what we were doing too.

Analytically, Dr. Seagrave described what takes place in the Exosystem of the Human Ecological System framework. The iterative course planning process was conducted among a network of interdisciplinary faculty, for the most part, without the "focal individual(s)" (the Students) being directly involved, along with a senior administrator to ensure institutional support. Albeit, occasionally, the faculty network would engage directly with students receiving the intervention, which is more in line with Critical reflexivity. Whereby, the faculty consistently engaged in acts of Critical self-awareness and reflection to modify their curricular approaches, by 
listening to the experiences, perspectives and voices of the intersecting identities represented in each of their classrooms. In fact, Seagrave offered an example of how he reflected upon and used Critical feedback from his students in a previous course he taught to inform his pedagogical approach to planning Race in the American Story. He recounted:

So when Dr. Shonekan and I designed the course, I was teaching a previous version of it myself and, and I, it's funny for me to think about now embarrassing, really, but I didn't I hadn't included any women authors in my syllabus. You know, the whole thing had no, no women authors. And so, you know, so a couple of students, couple of female students came up to me after class and, and said, you know, we just wanted to express that we think it'd be important for you to include some, some female authors in the syllabus. And so it was like, Yeah, absolutely. Yeah, you're right. I can't believe I'm not doing that. Right. So um, so you know, they helped me kind of Think through who to include what to include. And, and then I brought that to the table to when we were designing this the syllabus for the Race and the American story course, you know, that sort of thing. So, yeah, so we so we get feedback through students both informally that way. And then also, you know, with the evaluations at the end of the semester, they'll always, you know, comment about how ]it] went and what they'd like to see changed.

Professor Seagrave's reflexivity allowed him to enter a new network of colleagues with a different Critical perspective based upon his willingness to be reflexive about his teaching, by ensuring that RAS would be more inclusive of women, an identity he left out in his previous attempt to address race in the classroom. His actions fall in line with identifying relevant social categories and how intersecting categories interact or co-constitute in a CRT/I framework. Until now, much of the data reported are the perceptions of Drs. Shonekan and Seagrave, who give their perspectives as the architects of curricular reforms, which provide a rich and introspective understanding of the implementation process. The accounts of Dr. Stephen Graves further enrich 
their stories and provide us with the perspective of a collaborative faculty member who accepted the invitation to participate in RAS as a new curricular reform effort. Dr. Graves, began by recounting his invitation as a Black Studies professor joining his peers is Black Studies and Political Science, to offer additional readings and materials to the course around race and what he thought needed to be included. He described his perceptions this way:

...And what kind of, you know, reading should we put together in order for these students to have a successful you know, discussion surrounding race and America and everything else it is and it's really kind of focused on United States and the founding fathers and kind of how we got here type of situation. Leading up to the protests of 2015. And so it was just meant to be this kind of lens for which we could explore kind racial issues and how it even got to the point, and what kind of ideas and concepts and philosophical ideologies got us to where we are and everything else, but to have the students be confronted with these readings and discussions across the spectrum of history, race, culture, arts and literature and everything else. It is to kind of paint this picture of races of racial America. So, we all just kind of contributed readings that we thought were important in everything else, as someone who's in black studies and political science and everything else is I definitely wanted to make sure that I contributed Black readings, because a lot of the time these courses can just be Founding Fathers centric man [course] and it just becomes about Jefferson and Washington over and over and over and over and the same readings and the same, you know, excuses and ideologies by White people in these things.

A Critical Race analysis immediately highlight Dr. Graves role to contribute scholarly content focused on race in America to prompt successful classroom discussions. Additionally, he situates the committee's goals around notions of time and space as they were careful to discuss race within the context of its history in America, leading up to the protests of 2015. The committee sought to make race central to the course by examining 
historical social documents, structures and powerful influencers, (e.g., the constitution, the founding fathers, ideologies and philosophies), through the lens of race. In doing so, it would allow participants to increase their awareness of the ways in which race has been used to oppress people and both build and maintain systems of oppression pervasive enough to be a catalyst for the racial campus unrest of 2015. Graves discussions about the courses treatment of vulnerable voices, perspectives, experiences and presentations of historical Black writers through literature was perceived, as a way to counter American educations' historical tendency to teach around Whiteness (Whiteness as Property) and ignore the treatment of non-Whites and their positioning within scholarship or American society. Moreover, Graves' mention of his intersecting identities as a both a Black studies and political science Scholar and how it influenced his insistence to ensure that Black readings were added to counter the traditionally "founding-father-centric" approach to political science. His role as a Black male scholar in Black studies and political science certainly placed him in an underrepresented position of power to advance knowledge around the historical legacies of race in America. However, of importance to note, Dr. Graves was intentional with his scholarly inclusions and moved beyond even traditional Black readings and ideologies readily incorporated in some classrooms. To this end, he attempted to offer curricular options that would not normally be presented in a formal manner for the purposes of prompting a good classroom discussion; of which I interpret him to mean as intergroup relations dialogue. In fact, Dr. Graves explained his perspective by stating:

...And so I definitely wanted to make sure that other ideas got implemented and not just the traditional idea, you know, not just traditional African American readings. Everyone does Frederick Douglass you know, What is the Fourth of July ["What to the Slave is the Fourth of July"] and of course and okay, let's have Birmingham ["Letter from a Birmingham Jail”] and those readings of course, again, staple traditional readings. And so I definitely wanted to make sure that I contributed something that was going to be you know, discussion based..., that really added something different and something new. So of course, I went to Malcolm X man 
and I went straight for those readings and also contributed "Ballot or the Bullet" and some of these other things, what we want and stuff like that contribute to that part of the story, because it's heavily important and...you can't tell the story of America without you know, and race in this country without Malcolm X, the kind of readings of Black Nationalism, so I felt that vitally important that we include stuff as well. So, for the course, it kind of came along. I again, I think it was just an idea of we wanted to have discussion. We wanted students to have discussions, you know, and so the way that the class really started just the idea of how can we break these ideas into smaller 10-15, you know, 20 little section, you know, 20 student sections amongst the four of us, and we all kind of keep the section, and then we cover all the same readings that the students are all participating at the same readings, or around the same time, that we could really have a really good dialogue and a really good discussion about these racial issues, that was, that allowed the students really to direct or at least lead the conversation based on the readings.

Dr. Graves' pedagogical approach suggests a challenge to the notion of Whiteness as Property. In an American society where Whiteness has historically been established as a form of property, functioning at three levels: the right of possession, the right to use, and the right of disposition, it also impacts the right to transfer, the right to use and enjoyment and the right to exclusion (DeCuir and Dixon, 2004). Graves was insistent on disrupting the incessant control over American education by challenging the power dynamics that have prioritized and positioned Whiteness over Black thought through exclusionary practices. In other words, Graves sheds light on the fact that, historically, Black Nationalist ideologies have been excluded from the curriculum, while at the same time, pre-determined Black ideological thoughts from Dr. Martin Luther King Jr. and Frederick Douglass, of which, do not reflect the wide range of Black ideology or the Black experience that may be considered controversial. And, by avoiding that controversy, controlling the disposition of those who experience the education system. Dr. Graves suggested the righting of curricular wrong by breaking a cycle of 
exclusion with his inclusion of Black ideological thought likely to invoke deep, controversial, and uncomfortable discussions and dialogue amongst all students who attend Race and the American Story. I draw attention to his emphasis on wanting students to become immersed and engaged in deep dialogue to discuss the centrality of race and racism as a vital part of the construction of American society. This desire to incorporate, arguably controversial, race-focused content to invoke discussion speaks to the prominence of the nature of dialogue in advancing knowledge; of which was heavily highlighted through the literature of Paulo Freire (1970/1993) and on Intergroup Relations Dialogue. Graves's reported approach to his contributions to RAS, certainly helped me to understand how central race actually was in its construction but he also provided very rich data about his perceptions of both he and his colleagues' role in the implementation process. His, nearly, step by step accounts help us to understand the ways in which an interdisciplinary team of faculty might collaborate and infuse their own academic values into a curricular reform effort in the face of a campus's racial adversity. When asked to describe a bit more about the process of implementation, Dr. Graves explained that:

...we all got drafts of the syllabus ahead of time, on purpose about what we wanted the class to be, we were all asked to contribute, you know, a list of readings that we thought were important. And then to kind of put them in, you know, scheduled or timeline categories... we kind of move from, you know, the early revolutionary period into kind of reconstruction and everything else. So we were all kind of thinking of important reading from these specific time periods that were highly valuable, highly valuable, and that we think the students should read across and again, we all kind of have little specialty areas we had some, you know, strict constitutionalist, founding father areas with Dr. Seagrave and Dr. Justin Dyer, who really focused on kind of American political thought. We had Dr. Langley, who was big on literature [bringing] readings and stuff from Langston Hughes and from the, you know, the literature, and cultural experience. Same with Dr. Shonekan on the music tip to create and have the kind of musical listings and the musical element to the course. And then I do Black politics and Black 
political thought. So being able to put all of our specialties together into these readings and into the syllabus to make sure that it flowed right was important, but we probably battled that syllabus back and forth three or four times via email, making changes and making adjustments and figuring out, bringing up you know the timelines, because we're going to have four or five different sections and we wanted them all to be on the same readings at the same time.

I gathered from the above recapitulation, that Dr. Graves perceived he and his colleagues to be Critically reflexive about their contributions by paying attention to the social construction of social categories and how they operated within a historical context during the revolutionary period and reconstruction era. Time and space also resurface as attention was given to specific time periods and certain places in America where scholarly materials from American political thought and Black political thought can be discussed through the lens of music, culture and African American literature of those times. Such an exploration would allow students to pay attention to the voices, perspectives, experiences and presentations of those who have been historically oppressed and discover new meaning. It is also important to bring attention to the iterative nature of this curricular reform effort, as he describes the back and forth adjustments to the course syllabus to bring all sections into alignment, which was another important aspect of how this course was implemented. Specifically, Dr. Graves shared the committee's intentionality behind synchronizing all sections of the course and stated:

...there's also an element that students could take classes from other professors if they want to and you can sort of exchange for extra credit. If someone had registered in my class, they could be given a week or a couple of weeks, if they wanted to just take Dr. Seagrave's class, with Dr. Shonekan's class...that way they could kind of have a different discussion or highlight different discussions in different classes with different students, even though they were covering the same readings, they wouldn't really be missing out, and they will still be able to participate in the other sections if they wanted to. So, we really wanted to make sure that we're having, you know, a nice scheduled timeline that we're all following in different sections. 
But we battled that syllabus around maybe three or four times until we got it just right. We wanted it to be easy and accessible and put these things in, in a reader [Reader friendly manner] that wouldn't require students to have to pay for the book. So a lot of the things that we have were all online, and we found a nice little teaching, you know, teaching American history-org website. Justin had a lot of the founding documents and readings and stuff already compiled to make it easy for students. And then it was just about, you know, the assignments and how we really wanted this, you know, what was the purpose of the course and it was only a one credit course too so wasn't a full [3 credit course] [The course was] was one credit, was a one day a week, kind of, you know, one day a week for an hour like really just discussion-centered based. So, it wasn't like a Monday, Wednesday, Friday or frequently meeting, we literally met one day a week for one credit. And so, that was really supposed to appeal to the students too. So that they didn't really have to focus on all the homework, the homework, the homework, they could really just dive into the readings and having the discussion. And that really being the focus point versus the focus point being on just getting assignments done. So I can turn them in and get the grade... So by having it one credit by having you know, limited assignments, or highly discussion based, you know, definitely, definitely made a little bit easier, constructing the course wise. And so I think that going into it as a group, creating the course, the idea of having it being one credit, one day week, and kind of focus on the discussion and the readings and stuff really kind of made it easier for us and was really, you know, part of the purpose to make sure that the discussion and the conversation amongst students was really the focal point.

Dr. Graves' perceived that students would benefit from occasionally changing their classroom network setting (Microsystem) to experience the same course amongst a network of different people from varied social and racial groups, likely to have diverse ideologies around race and racism in America. Setting all sections of the 
course on the same synchronous timetable, would allow students the opportunity to place themselves in a more diverse intergroup atmosphere in case their course of record was more homogeneous. All faculty participants reported having 1) mainly Black students with varying social identities, 2) mainly White students with varying social identities, or 3) a mix of students from multiple racial and social identities in their sections during different semesters. They attributed that to students' initial awareness of the new course and their familiarity with each of the participating faculty. For example, some of the Black studies professors reported having more of their Black students who were already familiar with them because they had taken classes in the department or were majoring in Black studies. The same was reported by faculty for many of the White students in political science. All participating faculty reported having advertised this new course to their existing students in other classes, which was the likely impetus for them enrolling in the course under the professor they were already familiar with, heard about, and enjoyed. Therefore, the faculty planning teams' intentionality to create synchronous sections is noted as an opportunity for students to network within and between more than one microsystem at a time to influence their learning with a heavy emphasis on dialogue/discussion. Lastly, the committee was very intentional about the amount of out of class time students need to spend reading and completing assignments. Dr. Graves made it very clear that they did not want students spending too much time reading and doing assignments for a good grade and miss the substantive nature of the course; but rather, they preferred students read excerpts with a Critical lens and be more engaged in course dialogues. Interestingly enough, the course moved to a three-credit model shortly after the initial implementation. When asked if he or his colleagues experienced pushback or enrollment declines after moving from one to three credits, Dr. Graves shared his perceptions about this change as follows:

I particularly don't think any of the folks who I've been communicating with throughout this process have had any problems as far as students' concerns about going from the one credit to the three credit. I think if anything, it was beneficial in the fact as you were talking about credit requirements is to try to graduate during certain times, sometimes it's hard to plug in 
the need for a one credit course, you know, versus if they need a three-credit course, a three credit course would get you closer to graduation. You know, a one credit course is kind of in there, you know, somewhere in the middle, a student who takes it at a one credit level is really taking it out of interest more than a need. This course is going to help really get me you know, to graduation, whereas I think as a three-level credit course, you could get both, a student can be really interested in it and receive, you know, a full credit that will get them closer to degree completion. So, I think it was really beneficial and twofold in that way.

The perceptions brings attention to the ways in which institutions set in place aspects of their institutional culture, values and beliefs around how race/identity-focused academic coursework will be offered, if at all. It also speaks to the perceived motivations or deterrents possessed by students who use graduation requirement policies to guide and craft their schedules. To this end, Dr. Graves' account reminds us to consider the flexibility and opportunities within core curricular requirements/major-specific policies that might support or impede students' desire to enroll in race/identity-focused coursework that are often excluded from available or heavily marketed options. What I infer from this report, around culture change, is that practitioners who find ways to increase students' awareness of or support their desire to take race/identity-focused coursework, have an opportunity to help define an institutions' cultural values and beliefs around the vital role they place in all students' development and inclination to be advocacy-driven for social justice. An institutions' curricular policies that are favorable to the ideals of diversity, equity and inclusion and how it will be experienced, supported and encouraged can aid more faculty to take the bold approach the faculty in this case did to design RAS, in creating additional curricular reforms without administrative pushback.

Overall, the faculty in this case who implemented Race and the American story did not encounter pushback or administrative backlash from Mizzou officials during the process of implementation. Dr. Shonekan reported that given the administrative faculty roles she and others on the planning team held within their respective departments, they were able to approve the course within the department and cross list it through 
Black Studies, Political Science and the Kinder Institute without an issue. Likewise, Dr. Seagrave reported the implementation process to be fairly easy, much easier than trying to implement a degree or certificate program. He shared that the course was approved by the colleges' curriculum committees without a problem and he did not have to go through the faculty senate for approval. He also clarified that faculty could have taken the option to amend an existing course, changing its title and content to fit a reform effort; avoiding cumbersome implementation processes but they decided to create a new course altogether. He explained that:

...we actually didn't run into really any pushback from administration or department chairs about offering the course. Maybe that was partly because of the fact that the problem was so clear and there was such, such an appetite for trying to address it, that our department chairs and our administrators, I think we were just happy that we were trying to do something and were supportive of it. And the way we were doing it, too, you know, it was just as if I had designed a course, in response to the protests, intentions are just, you know, steady. If Stephanie and her Black studies colleagues had designed a course there might have been more opportunity for pushback or, or, you know, people having a problem with it for one reason or another, but because we did it together, I think that made a difference that, you know, people, yeah, there was more room for people to get on board basically.

Professor Seagrave perceived the absence of resistance to implementing the course, a result of the tense campus racial climate displayed via the protests of 2015. The problem within Mizzou's human ecological system was clearly fractured by racial unrest. The pervasiveness of racism perceived and felt by students and some faculty are a reflection of CRT's Permanence of Racism; whereby, Bell (1995) contends that in a CRT framework, the "realest view" requires one to realize the role racism has had and continues to have on American society unconsciously or consciously. Consequently, the faculty team who developed RAS at Mizzou realized the incessant role of racism amongst their students and became eager to fulfill-what Seagrave 
called-"an appetite for trying to address it." Under Critical analysis, one might also conclude that administrative officials may have recognized the institutional benefit to the university by being able to point to curricular interventions that help to calm the campus racial climate and remove the negative national spotlight, presumably damaging Mizzou's reputation. Such a conclusion is consistent with CRTs notion of Interest Convergence, where making change-for those in power-is primarily driven by the benefits to gain or gained from obliging to demands for making changes. Along these lines, Seagrave cautioned administrators at any institution who are faced with the responsibility to guide campus reform as he explained:

So, administrators I think sometimes tend to tend to think about like programs that can have, like immediate, some kind of quantifiable result that will look good on their resume or something right? Not all the time, but I think sometimes so they can be a little short sighted that way. And it's important to, to be aware of how deep the problem is. And, and to be, you know, humble in in knowing that hey, I as an administrator need to work with faculty, with students, listen to them, get feedback, and really do something sustainable rather than just trying to put a Band-Aid on the situation.

The professor suggests the importance of administrators, in particular, paying attention to the voices of the vulnerable, to be concerned with their treatment and to listen to their counter stories in order to move beyond short-term and episodic change that temporarily remedies a new crisis. He calls for administrators to, essentially, be more focused on how efforts actually make people feel rather than solely on what can be largely quantified and touted for personal or institutional benefit. What is also important to note from Seagrave in the second to last passage above, is his perception that Black Studies faculty alone might have experienced some level of pushback if they did not collaborate with faculty peers from other disciplines. His perception of how others at the institution may have reacted to a Black Studies-only, faculty-led intervention, speaks to perceptions of a lack of value in the academic contributions of Black academic scholars on their own accord. As if the content produced for consumption might not be deemed as credible or fit for people from all 
backgrounds and identities. While such perspectives of some individuals may have aligned with this skepticism, Dr. Seagrave's account reifies the vital role that a collaborative interdisciplinary approach to implementing race/identity-focused curricular reforms has on large-scale receptiveness and academic success. Therefore, in the interest of my concern for institutional transformation around social justice, I suppose that curricular reform interventions addressing race/racism and identity are more likely to gain large-scale receptiveness through the interdisciplinary collaboration of faculty. Although Dr. Seagrave's perception of how people may have responded differently did not occur, he did share that some fellow colleagues within his discipline seemed to have displayed some vague awareness or mystified behaviors related to his role in the course. He described it this way:

...I'd say subtle pushback. Mostly I've had support, so mostly support from other faculty and even enthusiasm from, you know, some of my colleagues, for sure. But there's been some subtle pushback, I think, and most of it not even not even noticeable to anyone, probably except for except for me, you know, just things like, you know, things like forgetting...that I'm teaching the course, you know, for whatever reason, when you're thinking about, like...the whole curriculum, or not prioritizing, you know, just kind of things that make it clear that some faculty maybe think it's not that important or not needed, or, you know, "why are we doing this?", you know, kind of questioning from other faculty like, "why again, are we teaching this course...why aren't they just teaching it in the Black Studies Department?" You know, things like that, where it's kind of phrased as a question or something, but really, it's kind of a subtle, subtle attack...

The subtleties Dr. Seagrave reported are reflective of microaggressions, the unsettled/unfavorable thoughts, opinions or behaviors that stem from an individuals' personal biases or unconscious biases held about a people or practice. The mere fact that some of his peers questioned why there needed to be a collaborative approach to RAS, speaks to their failure to recognize the Permanence of Racism by realizing the role race and racism has 
played in American society through their own discipline of political science. Said failure to recognize the role of race stands in contrast to Dr. Graves' earlier statements questioning how one can truly teach or learn about American politics but exclude race from the discussion. I have highlighted Dr. Seagrave's perception of his encounters with peers to bring attention to how White Privilege moves and operates, perpetuating the ideologies of Whiteness as Property and the Permanence of Racism amongst the faculty ranks. For example, imagine being in such a position of power that one can move freely throughout the faculty ranks, maintaining their privilege not to be concerned with how race and identity influence their discipline and to exclude the intellectual property of vulnerable voices. Their failed interests and actions perpetuate the notion of Whiteness as the standard of normalcy and superiority while infringing upon underrepresented groups' right to possession, use and enjoyment of a rigorous and inclusive learning environment. It is the pervasiveness of the aforementioned ideologies that develop and guide institutional structures of oppression that go unchecked and unnoticed by those in power and not directly affected. Thus, creating the discontinuities in race relations in American Society, of which, carried out at Mizzou.

\section{Towards Combating Discontinuities of Intergroup Relations in American Society at Mizzou through}

\section{Education}

The previous section of this chapter focused heavily on the perceptions of the process of formulating and participating in a curricular response to an exceptional campus climate issue at Mizzou. I presented the findings of my faculty participants-primarily-and reported my analysis of the data collected, to reveal their perceptions under a Critical Race Theory/Intersectionality lens, situated within the story of curricular reform interventions. I intentionally avoided reporting the findings on the intricate details of the purpose of the curriculum/assignments, the most valuable and important inclusions to the course or the actual counter-stories and perceptions of how the course addressed the discontinuities in race and racism, to allow the implementation process to remain central to the focus. Accordingly, I now shift our focus to the latter ideals to offer a clearer picture of the ways in which educational practices or an 
educational setting was perceived to combat the discontinuities of race and racism. I begin with Citizenship@Mizzou.

\section{Citizenship@Mizzou}

When asked about the purpose of the overall curriculum for Citizenship@Mizzou, Dr. Shonekan reported that it was to orient students to the expectations of the University of Missouri through the four institutional values that many people, before, never really knew existed. The goal was to move beyond traditional orientation methods of displaying information on a screen and running through the slides, but to become aware of how each value should be reflected upon in practical contexts. She explained her perception of how little people knew of or could expound upon the university values, saying:

...even if they could name them couldn't really talk about how those values, you know, how does the value of respect to have roots in how we live in our dorms, how we interact in our classrooms, you know? And so we felt that that if we could do that in two hours, with music, with engagement with the new students, then at least that was an orientation really, it's an orientation. It's not it's not a deep course pedagogy, you know.

Dr. Shonekan hinted at the aspect of the various microsystems students' network with by aiming to help students consider how the institutions' values influence the social interaction within their human ecological system at Mizzou. She also made it clear that this intervention was not meant to be a deep dive but rather an introduction to these ideals as a foundation for framing how ones' embrace of or failure to embrace the institutions' values uphold or disrupt the ecological systems' social networks. From a Critical perspective, I questioned the role race played in the presentation of anecdotes that represented each value. I was initially unclear of how this intervention could be considered a direct response to address the discontinuities in the campus climate mediated by race if the four values were the central focus of the diversity training course. Thus, to Dr. Shonekan, I inquired "would you say that that race was sort of a 
focus in Citizenship@Mizzou in how you sort of described the values of the institution and how you gave examples or anecdotes throughout, what would that be accurate to say?" She responded by clarifying:

Yes, I mean, it would be accurate to say because I started with "this is What happened at Mizzou, you know, a group of students felt that after Mike Brown, they felt that the university was not stepping up to the to the plate with making sure that the campus was a space that was welcoming to all," you know, and so we so we started there and then realizing too, that we had a lot of international students, we had a lot of LGBTQ community members, we had many, many different identities. So just keeping it with race may have excluded a lot of other concerns. And so we, we kept it like that. I mean, one of the faculty members on the panel, you know, her story was, I think she connected hers to respect, I think, and she talked about coming out, you know, to her family, you know, and she has a very personal story about, about her journey in a very orthodox Jewish family coming out, to coming-out to campus, you know. So as a student first, as a student at a different university and then coming to the university. So, race was what brought us there, what brought us in the room. And we definitely used it to project into other types of concerns that would be a problem at a university, you know, we created it not only after the protests of 2015, but also after a student had written some anti-Semitic stuff on dorm walls, you know, so we it wasn't just about race, it was definitely race that brought us in, but there were other things that we wanted to talk about, and we thought that the values allowed us to do that. Yeah. If you think about real quick, if you think about -I think the reason why I did that and why I always will do that is that you know, as bell hooks talks about this is not, racism is not just one thing. You know, it's part of a much wider umbrella of that comes from White supremacist status quos. You know. And so, when we think about sexism, when we think about homophobia, when we think about racism, when we 
think about ageism, all of it comes from a patriarchal, white supremacist framework. And so we shouldn't ever, I don't think we should ever, respond with just one thing. You know, we should understand that but it's much bigger.

Dr. Shoenkan's explanation above provided deep insights into how she, on behalf of the institution, sought to combat the disjointedness in race dynamics with an educational approach framed around campus values. First, under analysis, I note that her foreword to each training session called attention to the centrality of community racism as the impetus for requiring the intervention. She quickly, however, grounded the nature of the course within the theoretical framework of Intersectionality. Dr. Shonekan argued that it was important for Citizenship@Mizzou to not only address race to combat Mizzou's campus unrest, but to identify all relevant social categories and to give attention to the intersections of race and identity by examining the nuanced lived experiences of individuals. She proclaimed that the vitality of the course might be minimized if it solely dealt with race and failed to consider the intersections of racial and social identities; including religion and LGTBQ+ identities that were also under attack at Mizzou. With a CRT/I lens, Dr. Shonekan's perceptions drew attention to the Permanence of Racism, intersectionality, and a greater need for Individual Diversity Development. For example, Shonekan referenced bell hooks's perspective that race is a part of a wider umbrella that also includes ageism, sexism, homophobia, racism etc. grounded within historical forms of White supremacy and patriarchal ideologies and behaviors. Consequently, I interpret her to mean that the historical legacies of power held by White supremacists and male-controlled systems have upheld forms of oppression for not only Black identifying bodies but for varying social identities that may or may not be Black or of color. To this end, Professor Shonekan affirms that race-and the perceived racist incidents - was what prompted the need for the course, but it was important not to exclude the voices, experiences, and presentations of multiple social identities during the training. A huge part of this training session replied upon the personal counter-stories of presenters, to help people understand how ones' identity influences how well they are treated within spaces. Thus, by 
embracing Mizzou's core values, students can become good citizens by being conscious of their role during social interactions in any space for which they are a part of or, on the contrary, decide to perpetuate forms of dissonance and oppression. Either way, Dr. Shonekan sought to expose the students to presenters who might, for some, start the process of contextualizing the correlations between identity and the institutions' core values that ultimately lead to good citizenship behaviors and a more inclusive and welcoming environment.

With this in mind, Jacob Somerscales shared his perception of how he and his peers were impacted by one guest presenter who spoke candidly about one of the values. Jacob's rich perspectives of how effectively he felt the training course leaned toward combating Mizzou's social issues is depicted here:

There was one in particular where we had a guy named Reuben Philby, who was like a former football player at I think like Georgia, who was a grad student at Mizzou at the time, and he played guitar. And he gave the talk that day on excellence [Core value]. And it was a special session that was just for student athletes. So he, you know, Reuben is an amazing guy and he really got kind of like, into the nitty gritty of like, what it means to be an excellent student athlete and uphold values. And, you know, it was extremely motivational, you know, kind of style. And it was, I guess it was very indicative of just how important is to kind of meet people where they are, you know, and take a step toward them. where, you know, he was the perfect person to talk to those student athletes. You know, I mean, like, none of us were college athletes [The band and facilitators], you know, I mean, like, we played, like, you know, sports in high school or something like that, but he was the only college athlete that really understood, you know, who those people were and what, what they were doing. So, moments like that were really important. And that was, it's kind of one of those things where, you know, we were going through and some people 
were, like, falling asleep and stuff, and then he kind of like, you know, metaphorically, like, slammed his fist on the desk and was like, "listen to me! You know, I've been where you are, you know, you gotta take this shit seriously!" So, yeah, that was a really beautiful moment. And I think it was very effective.

Jacob's account of what took place during one session, draws attention to the speaker' s identities as a Black male, a graduate student, a former college athlete and a musician and how these identities intersected during his journey towards excellence. Jacob recognized the lack of empirical knowledge he, his peers and other faculty presenters held as athletes and noted the value of having someone to "meet students where they are," which is consistent with CRT/I's concepts of identifying relevant social categories, operating at the individual level to investigate how nuanced lived experiences inform social structures and vice versa, and paying attention to the experiences, perspectives, and presentations of vulnerable voices. Rueben's identities and experiences were nuanced and were used as a way to promote students' inner aspirations to achieve excellence, based upon how he had to perhaps challenge the process and navigate success while carrying multiple intersecting identities. Jacob reported Rueben's approach to be "beautiful and effective," even to combat the dispositions of those students who may have been initially uninterested.

To further probe perceptions of effectiveness, I explored how participants in this study perceived those needing the course most for the institution to combat around racial and social unrest. One student participant, Zoey Romyn, explained:

Um, I think yeah, I don't know, that's tricky because it, it talks about a lot of identity. So it's, I don't know, any identity who wasn't necessarily on the stage I think should be bearing witness to that. So people who are cisgender people who are straight, white people, especially, who have yet to acknowledge their privilege, like just people who I feel 
like having the story behind like statistics or being able to put a name to a face is really important in terms of imparting that knowledge and understanding.

Zoe's perception about who needed the course most centered around identity and privilege. Analytically, she believed that anyone who carries unchecked privilege based upon their identity status, should take the training to listen to the voices, experiences, perspectives and presentations of the vulnerable. Zoe also perceived the experiences of people who have counter-stories behind the existence of generalizable data about them, valuable to the advancement of knowledge. Whereby, consistent with CRT/I's attention to how traditionally marginalized people are situated within positions of power and knowledge production.

On the other hand, I asked the participants "how do you perceive the course's instructional strategies, assignments, activities and projects as fulfilling its purpose to create learning opportunities around race and racism? And how well do you think the course has influenced behaviors to combat, advocate or face race and racism?" Jacob was a bit perplexed by the question and offered a flurry of thoughts that addressed a number of possibilities. For instance, he suggested that Dr. Shonekan's optional reading and music list for students to review and write about for a door prize might have been conducive to the learning outcomes. But on the contrary, he supposed that students might not draw out the true meaning from what they read or hear, because they are not yet "cured" from the problem after going to frat parties and hearing rap or hip/hop music all the time. His perspective on current and future effectiveness was:

...it's, it's, it's tough to say whether that stuff was really effective or not. I think, I think it has all the potential to be effective. But it again the responsibility kind of sits on the consumers' shoulders to treat it with a Critical mind and, you know, a certain level of introspection I don't know, we had we had talked about also doing a, like a follow up thing. So like having people go to the first session, and then we have a second session later on, that is also 
required that was, you know, basically just like, how far have you gotten in this journey, how are you enacting these values in your life?

Jacob's perceptions highlights the challenges facing the institution in effectively combating Mizzou's disjointedness in intergroup relations, primarily ignited by racism. Of importance to the CRT/I analytical framework, is Jacob's perception that the burden of student effectiveness did not solely rest upon the instructional strategies, activities and assignments offered, but also on the student's mindset. He argued that students needed to treat the training course materials with a Critical mindset and that they needed to be reflexive by constantly being culturally self-aware and reflective about their own positionality in notions of privilege and oppression as they discovered more about race and identity dynamics. To this end, Jacob and Dr. Shonekan's team had hoped to implement a subsequent required training course to assess the nature of their cognitive attitudes and behaviors in relation to the core values espoused during the initial training, but this did not materialize. Moreover, Zoe responded to the same question with rich information about her perceptions of Citizenship@Mizzou's pedagogical strategies on effectiveness and how she perceived the course to be more effective given the nature of the subject matter. She very kindly but firmly stated:

Um, well, the style of the course was $100 \%$ lecture, like there wasn't really any engagement component of it and because it was such a large lecture hall, I don't know that any of the lessons were necessarily imparted successfully. Just because, I feel like a lot of those conversations need to be more individual, to like address specific conversations or specific questions from people who are struggling to identify their privilege and not really understanding because they haven't seen anything other than what they've always known. And so I think that those conversations and specifically conversations about race and understanding the implications of race, I think those conversations need to happen on a much smaller scale, like on a more individual basis. So, I don't know that citizenship 
necessarily accomplished that just because it was only lecture, like there wasn't that much engagement happening with individuals in the audience...

Interestingly enough, Zoe perceived the training course to lack engagement and labeled it "100\% lecture," despite Jacob and Dr. Shonekan's reports of their attempts to elicit engagement with a live musical band to play the songs that would be discussed. Zoe believed the course's focus on race and identity-very controversial and heavy subject matter- needed to be addressed in a smaller and more engaging space, where people grappling with ideologies and experiences presented can interactively dialogue. Under analysis, I took her comments to align with CRT/I's espousal to, identify relevant social categories within historical contexts to determine how they operate and to work at the individual level to investigate how nuanced lived experiences inform social structures and vice versa. Additionally, it is important to note that Zoe shed light on the idea that some of her peers have not recognized their privilege because they have not had to do so, which aligns closely with notions of Whiteness as Property, as they have been afforded the right to said disposition; one that diminishes or excludes the contributions and experiences of non-dominant racial and social groups. Furthermore, I interpret Zoe's response to strongly advocate for small-scale intergroup relations dialogue opportunities as a vital part of the learning process, to more effectively address the discontinuities in race and identity prone to arise. In essence, I draw attention to Zoe's perception of the role special dynamics and having interactive pedagogical strategies that intentionally shape the educational atmosphere, have on making it more conducive towards combating issues with intergroup relations. Along these lines, but beyond instructional strategies, Zoe continued to share her perspective on the course's learning materials being surface, intermediate or in-depth, as she explained:

umm I would say very surface level in terms of like How the content reached them, which was unfortunate. Because I think it was really dependent on how much the individual wanted to engage. And if they weren't me who was sitting in the front row, they definitely had a choice not to engage or not to pay attention at all. So, I would definitely say surface 
level. And then yeah, going back to just like how I would amend that and make it more engaging for those who are partaking in it would just be smaller, smaller, like discussion groups, and minimal lecturing because I feel like it's, it's through conversation that we're going to actually make progress and get people to understand and if people are just being told how they're supposed to behave alongside 699 [Random estimated number] other people. It's a little, it's not the most successful tactic.

To me, Zoe's assertion about the surface level nature of Citizenship@Mizzou sound counter-productive at first but earlier in this chapter, I reported that Dr. Shonekan clearly stated that this intervention was not meant to be a deep dive but rather a foundational introduction. Despite Zoe's discontent with the instructional method, learning material and spacing, she reported herself to be engaged in the front row. It was her perception that the meeting space was too big to hold the attention of her peers, several rows behind her, or to adequately discuss such content to any degree of significance. Analyzing this Critically, forced me to consider the was in which her peers' disposition or uninterested nature upholds the notion of Whiteness as Property, which simultaneously perpetuates a Permanence of Racism-sometimes unconsciously and for others it is done so consciously. On the other hand, Jacob's perception of the training course's learning materials was:

I would say no more than intermediate. You know, I would say it was, you know, it was focusing on these values about how to, you know, how to be a good minister to students you know. But, you know, ultimately, those are four values that were invented by a White institution, you know, and that are not really... No, I mean, there's a reason that like the word diversity was not wanting to be used, you know, people didn't want to touch on the topic. And, and make it so precise. So yeah, I mean, I would say it was intermediate. We did as good as we can, you know, talking about this kind of stuff without getting too hard edged. Regretfully. 
Jacob recognized the goodwill aspect of students learning to be good to each other but under my analysis, I consider his personal theory about the creation of the values to be much like that of a Critical Race Theorist. His attention to the fact that White people conceived the institution's four values and touted them as the central focus to remedy Mizzou's racial unrest, reaffirms the notion of Whiteness as Property. Along these lines, Jacob called attention to the discouraged use of the word "diversity" to describe the course, as a Critique of Liberalism; episodic change that is slow-paced and carefully implemented to avoid resistance from dominant groups in power. Because of the institution's failure to precisely address "diversity" in the description and throughout the training in a "hard-edged fashion", he concluded the education level of Citizenship@Mizzou to be intermediate at best. Jacob did, however, confidently state that he and the other facilitators did their best with what they had to make some progress but regretted not being able to be more direct with the subject matter. In framing the dilemma around indirectness and a lack of depth on diversity content, earlier in this chapter I reported that the name change was initiated by a Black student's suggestion to Dr. Shonekan. On the other hand, Jacob viewed this as "people not wanting to touch on the topic;" it is likely he was referring to university administrators who did not want to ignite resistance in the White students taking the course. Naturally, one might assume that Jacob was inaccurate in his assessment given the previous explanation of how the name change came about, but it is important to note the reasons behind why the Black student may have felt uncomfortable using such direct terms. To this end, consider the nonmonolithic reality of Black people who do not feel or think the same on every topic and the tense conditions Black and other traditionally oppressed identities might feel when they know that their White peers are likely to focus narrowly on and blame them for being required to take the course. Thus, Jacob's statement that there was a reason why the word diversity was not used, is not limited to who called for its non-use but rather the fact that the atmosphere was not perceived conducive to use it without more social unrest. I believe it is important for practitioners to make note of the nuances in cases such as this; the diversity of 
perceptions about race/identity dynamics are not simplistic, nor do the individuals who hold them embrace a one-size-fits-all prescribed approach to reform.

With Jacob and Zoe's stories in mind, I explored the perceived preparedness and ability to facilitate the course around race (identity) of those facilitating the training. Zoe replied:

I think that the professors who were there were really passionate about what it is that they were talking about and talking about their stories and their experiences. So, I think that in terms of faculty and staff, I think that it was one of the best groups that could have been present. I just don't think that the strategy of imparting their knowledge to a room of 700 [Overestimated number] people was the best.

Zoe gave much credit to the preparedness of Citizenship@Mizzou's facilitators and valued their counterstories and passionate nature towards the treatment of vulnerable voices and experiences, despite her unfavorable perceptions discussed above. An important detection here is the difference between those who discuss or teach around race/identity dynamics passionately, versus those who might do this work out of undesirable obligation. Although-not solely focused on instructor preparedness -in a more detailed manner of reflection, Jacob described his perceptions about Dr. Shonekan's preparedness by reaffirming the musically creative aspect of her teaching style with a few examples, while pushing back on how he perceived the university to be controlling the intervention's packaging. Jacob stated:

So, you know, in hindsight, I don't think there's anything we really like could have done because our hands were tied by the university. But, you know, from kind of a structural standpoint, I think that the university you know, the people above Dr. Shonekan should have been more accepting of, of that kind of teaching. You know, I mean, if we would have talked about, you know, I mean, like, for instance, yeah, we played you know, what's going on by Marvin Gaye. And we kind of talked about, you know, the values in that song and what he's 
talking about a little bit, you know, we did like a mashup with that and john Lennon. Imagine, you know, to kind of say like these songs, they were both actually written in the same year. And it was kind of two guys from, you know, obviously an American Black man and a British White man talking about what they see in the world around them. We touched on those things very little. And it was, I think it was good, but I would have liked to have talked about, like, you know, I mean, numbers and stuff. And I mean, like, this is, you know, when, when Marvin Gaye was like writing the song, I mean, he's like, you know, an American man who is like, faced a lot, you know, I mean, he's, I think he's from Detroit. You know, which has like a, just an insanely deep, like, redlining history, talking about stuff like that. I mean, really talking about like, the nitty gritty about what's going on, I think probably would have been more effective. Because that's something that people, those, those kids that, you know, frat kids or something like that, you know, grew up in the suburbs or something. They're not taught that in high school. They're not in there. Not going on the internet, usually and searching for that kind of information. And I think that's what probably the race in the American Story class was trying to get at. But again, when it's a class, that's an elective like that, you really only get the people that probably already know these things. You know, I would have liked to integrate a little bit more of that. You know, those difficult conversations into the citizenship courses.

Jacob's answer quickly validates the level of preparedness to have carried out the work he, Dr. Shonekan and others did by stating that there was nothing else they could have done to go more in depth, given his belief that their hands were tied by the university. Using Marvin Gaye and John Lennon's music to examine the lyrics in relation to the values of the course was a pedagogical strategy used but Jacob felt a limited endorsement of university officials to delve deeper into-what I know to be-a Critical Race Theory/Intersectionality analysis. In this case, I am using CRT/I to analyze Jacob's response but also drawing 
attention to how Jacob's desire for an improved curricular approach is essentially a desire for a CRT/I pedagogical approach. For example, Jacob identified the relevant racial and social categories of which Marvin Gaye and John Lennon were a part of and he recognized the historical context of one growing up in Detroit during a specific time period in a space with a deep redlining history. Additionally, he remarked about the American education system's exclusion of opportunities to advance knowledge with such information; because of this, a population of students will never even know to seek out ways to explore race and identity or possess enough interest to pursue educational opportunities to attain it. Nevertheless, these curricular exclusions and rights to hold such dispositions are consistent with Whiteness as Property, which is why Jacob believed it would have been more effective to have deeper intergroup conversations in Citizenship@Mizzou. It appeared to me, that Jacob perceived the university's ultimate control, to somewhat stymie the freedom to delve deeper, ultimately limiting the effectiveness of the training course as an intervention to the campus unrest it was meant to reform. Such limitations in Citizenship@Mizzou did, however, pave the way for a faculty-led institutional curricular response called Race and the American Story, which has been offered as a one-credit or three credit hour course.

\section{Race and the American Story}

Race and the American Story was considered an outgrowth of Citizenship@Mizzou and was meant to delve deeper into the discontinuities in the intergroup relations in American Society and Mizzou, mediated by in depth dialogues examining race and racism. I continued to provide rich accounts of this study's participants under a CRT/I analysis, to help us understand the perceptions about the institution's shift towards combating the social and racial disjointedness of Mizzou and how participants perceived their peers or their own experiences creating, teaching or enrolling in the course. To begin, Dr. Shonekan offered her perspective on the transition from Citizenship@Mizzou to Race and the American Story by describing its overall curricular purpose this way: 
Similar to citizenship at Mizzou. But this one was clearly about race you know, we have had a few people say, you know, what about gender and the American story what about you know, LGBTQ and the American story and we definitely agree that there are all kinds of things that we need to do along those lines. But this wasn't an orientation course this was a course-course you know and so for us in academia, we like to tell, we tend to take one thing and drill down on it and for us, it was important to drill down on race, which we couldn't do in Citizenship@Mizzou, but we could do it in this course. At the time, we created it, there was so much you know, Twitter was [Inaudible], you know, it was the 2016 election and you know, all that stuff was going on, if you remember that, and we wanted to go beyond the tweets to give it some deep connection to the history. So, that was the purpose of resetting the American Story, it was definitely to bring context to our conversations about race to show the historical context and to even connect it back to our campus experiences.

Immediately, under a CRT/I analysis, I recognized the reported focus on race. Dr. Shonekan was very clear to note that while she and her colleagues agreed that other aspects of ones' identity are important to explore in addition to race, this course needed to focus on one primary area in order to do a deep dive into its complexities. As an effective pedagogical strategy, she set out to move beyond Citizenship@Mizzou's broad and foundational knowledge approach to intently focusing on race, given the tumultuous climate in America and Mizzou during the time of the case; all of which stemmed from the discontinuities in race relations. Another analytical take away is Dr. Shonekan's mention of Twitter, which I interpreted to have compounded Mizzou's campus climate issues along with the racial unrest following the presidential election of 2016. The reference to Twitter speaks to the Techno-subsystem in the adapted Human Ecological System theory; it considers the ways in which social media, mobile phones, television, and other forms of media influence individuals. Dr. Shonekan reported wanting to move beyond Twitter posts and what was happening nationally as reported by the media, to provide historical context. Her desire to situate the 
complexities of race relations within a historical context, reinforces CRT/I's insistence on examining the ways race has historically been used to inform social structures and create systemic forms of racism and oppression. Said approach, paved the way for the course to intellectually step outside of the Mizzou case in order to consider the Permanence of Racism upheld over time that ultimately led to what students experienced at Mizzou. Likewise, RAS' co-creator, Dr. Adam Seagrave, stated his perspective of the course's purpose in this way:

You know, basically it was to create learning communities among people who, who wouldn't otherwise get together and learn together about these topics and the history. So that was the goal. I mean, we wanted to get students together, who wouldn't maybe otherwise be together in the same classes or hang out with the same people or have the same friends. And we wanted to get them learning the history of race in America and the conversations that have taken place about race in America throughout our history. And to encounter that history in a really deep way to talk about it with each other and with other students. To kind of immerse themselves in the history. So that that was the goal. So, create a learning community and broaden people's perspectives. And in a couple of ways, I mean broaden their perspectives because they're talking to other students who, whose perspectives are very different from theirs, who they might not otherwise talk to outside of class. And then also you broaden their perspectives by showing them that the things they see around them, the issues that we see today, have a really deep history, and they've come from somewhere, right? They didn't just appear today, like they come from somewhere. And so I think that perspective is really important. And we wanted to give students that perspective.

Dr. Seagrave instantly spoke to the intergroup nature of RAS' goal to create multiple learning communities. Whereby, students can academically network with multiple microsystems comprised of a diverse group of peers, which is consistent with the networked model of the Human Ecological Systems theory. The idea of 
creating a learning environment for people representing multiple identities to intermix is on par with the literature presented on intergroup relations dialogue (Zúñiga, Nagada and Sevig, 2002). Dr. Seagrave’s perspectives on the necessary pedagogical strategies to employ; for example, the use of historical documents and intergroup dialogue, follow CRT/I's philosophy regarding the exploration of relevant social categories (race) within a historical context to dialogued about the role it played in structures of American society. Additionally, their strategies operated at the individual level to investigate the nuanced lived experiences of traditionally marginalized groups and considered how these groups have historically been positioned in notions of power to advance knowledge or have had to challenge power and fight to dismantle systems of oppression. Dr. Seagrave's response aligns with individual identity development principles, as he planned for students to share and become deeply aware of other perspectives different from their own; perspectives they have not had interest in or had to grapple with. Thus, creating an opportunity for discovery and awareness about other racial identities they otherwise may have never networked with in any social or academic setting. In a related but different fashion, Dr. Stephen Graves provided his perspective on the overall purpose of RAS's curriculum in combating Mizzou's campus climate issues by going in depth with specific examples of course content. I decided to pare apart his initial responses from those that led into the intricacies of the most valuable contributions to the course, to focus separately on the rich information shared. Dr. Graves first stated:

And the purpose of the overall curriculum was to be short enough readings. So, not no full book chapters, we wanted short enough readings, short enough excerpts so that students could fully not have to worry about the highlighting and they could really read and concentrate on content and not be overwhelmed by page numbers and lengthy readings. We wanted a nice short and compact reading, so students can fully engaged and fully, you know, could reread it if they wanted to in five or so minutes. And I think it fully developed into the actual readings and not be overwhelmed by page numbers and highlighting and 
making sure I have these certain things. We wanted to make sure that the concrete fundamentals/beginnings were always there at the very base. So the constitution and Articles of Confederation in the Bill of Rights and Jefferson's notes and Jefferson's letters on the constitution in the early draft, we really wanted to get a, you know, we want to deal with some factual knowledge, we were trying to be intellectuals and everything else. That is right. We wanted the ideas to make sure that students had a firm grasp of the opinions of the founding fathers and Kant and know exactly what the constitution and Bill of Rights says, so that we're not getting off into some idealistic perspective about what the country is, [but] that we're dealing with living documents and the words of the founding fathers, that we have some concrete, you know, facts about the purpose and goals, or what America was supposed to be and the ideas going into it. So, we want short, compact readings and everything else it is, we wanted to deal from a factual and again, like I previously stated, and we wanted to diversify those opinions and stuff as well. But obviously, there are just some readings that you can't do without if you're talking about the story of America. So like I said, with the constant, and, and, you know, Jefferson's notes on slavery and early draft of the constitution, the Bill of Rights and things like that, you know, the Emancipation Proclamation and, and those readings that the, you know, the debates between Douglas and Lincoln, those are some foundational, racial, you know, discussions that that we had to have, you know, we wanted to make sure that we had those very important basic documents that really tell the story of the founding fathers and the development of America racially and the discussions and debates going back and forth. So that was important to have those founding debates about, you know, Africans and slavery and the founding fathers and everything else it is. 
As a pedagogical strategy for optimal course engagement and learning outcomes, Dr. Graves emphasized the planning teams' perspective to assign short readings to avoid overwhelming students with lengthy reading assignments that might deter their ability or interest to focus fully on the nature of the content. I think it is important to note the pre-conceptions on student receptiveness during the development of course assignments, to increase the likelihood of wide-spread interest and classroom engagement about the subject matter. The faculty found it more beneficial to cover more of America's founding documents, to offer historically diverse perspectives, by using shortened excerpts rather than spending too much time on one historical ideology. Analytically, the said approach would allow the students to use historical context to consider the ways in which foundational American documents, written by the founding fathers, have addressed race and influenced the lived experiences and institutional structures of non-White American citizens. Essentially, Dr. Graves reported the significance of Critically analyzing the documents and debates of foundational figures to examine the role of race during the founding of America and how the country's systems, values and peoples' ideological perspectives sought to undermine various forms of racial oppression. He and his colleagues afforded students a formalized academic space to be deeply engaged in dialogue as intellectuals, analyzing and being attentive to the historical nature of power dynamics, situated against the backdrop of philosophies and experiences of the founding fathers, President Lincoln, peoples of African descent etc. The approaches described, will allow students to come to understand the Permanence of Racism in America throughout history and the role Whiteness as Property played in determining who could be afforded the rights to possession, use and enjoyment of knowledge, property, space, freedom, happiness etc. or to hold superior dispositions of power and control. In fact, Dr. Graves referenced the importance of presenting students with opportunities to weigh the different perspectives of racial groups over time, as people from oppressed groups began to form opinions and speak out against their mistreatment. In a very thickly described manner, he continued by stating: 
...But once we get past that, it was really about diversifying the ideas and diversifying opinions. And so we want you to start getting the Frederick Douglass, getting the Reconstruction, we start adding in Booker T. [Washington] we're adding Dubois [W.E.B.], we start adding these other ones, because now Blacks will have voices and now their perception of America starts coming into [play]. And we would definitely want to make sure that we highlighted a difference of opinion and a different perspective, around the same time periods as the founding fathers, so when we start getting into, you know, Frederick Douglass and the Dubois and Booker T. and some of these others, and Alice Cooper and some of the others, now we're really trying to update the story and the impact that those founding documents that we started with, with the Constitution, and now we're trying to see how those things are working and how they're being implemented in real time, over time, and everything else it is.

My interpretation under a CRT/I analysis finds Graves and his colleagues' attentiveness to the treatment of vulnerable voices, experiences, perspectives and presentations of the Black scholars he named, to fit nicely within the framework. Additionally, their curricular approaches described, continued to point at the necessity to operate at the individual level to investigate the nuanced lived experiences of Black scholars. However, much like a CRT/I analysis, it was important to do so within the bounds of time and space, while examining the influential role of historical documents on race in American society. Multiple faculty reported the vitality in evaluating the historical legacies of racial discontinuities in order to fully understand the deep roots of racism that reared its head at Mizzou. In fact, Dr. Graves excitedly gave an additional breakdown of his perceptions of RAS' assignments and the intended outcomes for student learning. He went on saying:

And so, being able to tell the story of the founding fathers first, but then be able to tell a story that African Americans are experiencing really helped a lot and bringing the class discussions and stuff and really bringing the purpose of the course together, right, because 
as you know, what things were facing at Mizzou and the things we're facing in the in the country. When we're talking about White people [They are saying/thinking] "we're supposed to be equal, what are Black people so mad about? It's not like that anymore," right? "slavery is over with, get over it" type of a thing and so these discussions after these readings are coming up, we are saying well 1 ) it was never equal and 2) even 100 years later we're talking about slavery ending and we're still not having equality and there's still no racial justice. And so we're trying to paint the picture of why these things happen, why these racial incidents keep happening, why they happened at Mizzou in particular because this is an issue that clearly has never been dealt with properly and has never been addressed in a way to bring the country and bring these racial things together. So we're trying to show that process you know, going up, so when we started getting to, you know, 1776 / 1762 or whatever else it is and we get to the letter from the Birmingham jail in 54-55. And we see that we're still having the same problems. Students can trace the students can trace the intellectual and philosophical and the ideological thoughts and opinions from 1760, all the way into 1960. And you can see Mercy [Just Mercy, 2019 film], right like, these, these same racial instances and ideas keep presenting themselves, even though the opinions are somewhat changing, and the people are somewhat changing and the time to changing. But the, but the elements of racial frustration have not changed and are not changing.

Beneath analysis, I surmised Dr. Graves' reference, of first beginning with the forefathers and foundational documents followed by the written and current voices of African American experiences, to champion the vital role of counter storytelling. Coming to understand the counter-stories over time, shed light on the Permanence of Racism in America and how it has never been fully addressed in subsequent spaces like Mizzou, which provided the basis for the racial unrest in the Mizzou case itself. Graves' perception was that we continue to see the same racial problems over time despite some people and opinions changing; this 
persistence, yet again, reinforces the notion of the Permanence of Racism that so desperately needs to be addressed. All of which is not largely incorporated or dialogued about in America's current education system. The notion of dialogue and/or discussion-as it is sometimes used interchangeably by some-was also validated by Dr. Graves as he reported-above-that hearing the counter-stories of African Americans' experiences within a historical context helped the class discussions and brought together the overall purpose of RAS together.

Dr. Seagrave supports Dr. Graves' mention of the usefulness of discussion in the learning process for RAS. His perceptions drill down a bit more into the faculty planning teams' perceived effectiveness of using dialogue/discussion. He stated:

You know, I think, from a pedagogical perspective, the most important thing is, is having discussion in class, I think class discussion. So I think by and large, we've tried to make it so that this is not a lecture course, that this has got to be a course that's largely based in discussion interaction. That's been really important. I think, in terms of readings, it's been important to include, you know, I mentioned before, including female authors, and just including as broad of a range of both perspectives and demographic backgrounds of authors as we can. And so, so it's been important to include, and, you know, for example, you know, Ida wells for the Lynch Law book [Lynch Law in all its Phases], and, you know, Anna Julia Cooper, and Francis Harper and some of these, these writers that most people don't know, but who are really, really important. And so, so that's been really important. And I think the combination, too, in terms of what we include. So combining those, those authors and authors like Malcolm X and W.E.B. Dubois, combining those authors with authors like Thomas Jefferson and Abraham Lincoln, you know, so having both White and Black voices, you know, kind of conversing with each other in some way throughout American history. I think that's been important too. 
Dr. Seagrave's promotion of structuring a discussion-based course, grounds Race and the American Story within the intergroup relations dialogue framework presented in significant detail through Chapter Two. He also re-references his inclusion of women authors while validating Dr. Grave's advocacy to expose students to multiple perspectives about America's racial history by including the voices of scholars who can provide the nuanced counter-stories within a historical context. Specifically, Seagrave referenced the value in introducing students to the scholarly works of Black voices of whom many have never heard of but offer significant counter-stories and perspectives that can advance knowledge. Another clear display of the committee's actions to consider the ways traditionally marginalized people have been positioned in knowledge production and how they have historically challenged power dynamics. Analytically, Seagrave thought it valuable to examine the discontinuities of race-particularly between Black and White peopleby analyzing its role in the development of American cultural values, that have shaped the racial and political ideological perspectives and tense intergroup relations of Black and White people over time. Much of the findings I have reported thus far has been heavily focused on faculty perspectives; so, what are students' perspectives about Race and the American Story and Mizzou's educational attempts to combat the discontinuities in intergroup relations stemming from racial unrest? Both Luke Davis and Ida Campbell-Jones were enrolled in the course and provided some thick descriptions of their perceptions about RAS.

Luke Davis was asked to give his overall perceptions of how the course's instructional strategies, assignments, activities and projects fulfilled its purpose to create learning opportunities around race and racism, and how well he thought the course influenced behavior to combat, advocate or face race and racism, he provided a large amount rich information to help us thoroughly understand his perspective as a White male student engaged in Mizzou's curricular intervention. I have done my best to break up my interpretations of Luke's responses - to the questions above- under a CRT/I analytical lens, while continuing to thread the networked model of the Human Ecological Systems theory as necessary. Luke first began by addressing his general perceptions of RAS by passionately sharing: 
Um, so I think that I was really lucky to be instructed by Dr. Shonekan. And it being, it being sort of her brainchild, right, was really important to me. And selling. I had an incredibly good experience with her because I think she is really understanding and kind of a meet-youwhere-you're-at kind of teacher. So, I think that the coursework, the course load was, was really good because it wasn't, it wasn't too much for a one credit hour class. It's usually just a couple of readings every week that we would then come back and talk about, and then probably two or three different projects that we did throughout the course of the semester. So, the course load was really not onerous. And it was it was really, it was a good; it was a good setup.

While I did not associate a Critical race analysis to the above response, it is important to note Luke's admiration and appreciation for his Professor, Dr. Shonekan, who he felt was understanding and relatable to students. He described his learning experience with Dr. Shonekan as incredible, touting his appreciation for her well-balanced construction of course assignments that did not leave him feeling overburdened with a ton of reading or homework assignments. Luke's account, validates earlier participant reports of the faculty's insistence on assigning modest reading assignments to allow students more motivation and comfort to read the short excerpts provided and engage in classroom dialogue. Along these lines, Luke dove deeper in his recollection to share a wealth of additional perspectives germane to this research study. He shared that:

...it was it was really just like a free-flowing kind of conversation. And we had really genuine and honest conversations and we felt like it was structured in a way that we can trust our classmates, that we got to know our classmates and that we formed a little community where we can be very open and honest about our experiences and about our opinions. There wasn't any conflict, like I'm sure there was, there was plenty [of times] where we disagreed on things or where there was pushback. But it was by no means just like an echo 
chamber. But it was a an environment that was super conducive to open and honest conversation and conversations that I had never had the experience of, nor did I have again at Mizzou. So yeah, I think we, I think it had a really, I think it had a really powerful setup. Because not only were we examining the history of race in America, and how that impacts each of our experiences and, and the way that our society is set up today, but we're also you know, we're seeing it in the big systems manner, and historical context and contemporary context. But we're also hearing about our year's experiences with institutional racism. So, it became, you know, not as objective, right? Because it will get into these academic spaces where you're looking at systems and it can be really different systems of oppression and, and the ways that, that Black and brown folks are oppressed in our country and see it as kind of removed from yourself and not, and especially as a White person and not relate to it, and how it impacts that you know, and love. And so, I think this class really allowed for that, because you were sitting across from your classmates or sitting across from your community members and hearing their perspective and looking into their eyes as they, as they told you. This is my limbic hearing. This is how I'm being treated on this campus, or this is how, you know, I have experienced things in my life beyond school. And that was extremely powerful. Extremely impactful. And it yeah, I mean, I think the class design, like, I wish it was more. I wish it was a three-credit hour course that we could meet more times a week because I think it was so important. So yeah, I think it's been a really, really good job in the way that it was structured and the way that it attempted to address the things that I wanted to address.

From an analytical perspective, Luke expressed his pleasure with the construction of a social network that created a safe and trusting microsystem to elicit productive and engaging opportunities to advance knowledge. It felt good for Luke to be in a comfortable space to directly discuss race, during a time when 
many people at the campus were on edge, longing to heal and experience change. The RAS course exposed Luke to the diverse perspectives and counter-stories of his Black peers in the course, to hear and discuss subject matter he had never been afforded the opportunity to learn prior to his enrollment. He used words like "trust," "open," and "honest" to describe the values for which he attributed to the effectiveness of the intergroup relations dialogues on learning outcomes and improved social relations. Luke's mention of never having experienced another course at Mizzou like RAS again, speaks to the uniqueness and rarity of a course dedicated to the analysis of racial dynamics throughout American history and its impact on systems of power and oppression. It also reminds us of how Whiteness as Property strips away and determines the nature of intellectual property, how it will be distributed, and what will be excluded in order to maintain ownership over the patriarchal narrative that names and defines how people should be viewed and treated in America. In fact, Luke referenced how RAS allowed he and his peers to examine the historical and contemporary contexts of race in America to understand the Permanence of Racism woven throughout history, that provided the platform for the institutional racism experienced within Mizzou's human ecological systems leading to the time of the case. Analytically speaking, Luke identified relevant social (racial) categories participating in the course and expressed how he perceived their involvement in RAS aided in the process of learning. Namely, he shared how his perspectives and experiences in American systems as a White identifying man, have been very different from the counter-stories expressed by his Black and Brown peers. Consequently, he perceived the positionality of traditionally marginalized groups to be instrumental in the advancement of knowledge, because he valued the contributions of their voices, experiences, perspectives and presentations. It became clear to him, that what he learned from listening to the counter-stories and engaging in classroom dialogues, were experiences that he and White people in America have never had to grapple with and in some cases, have never realized the full extent of what Black and brown people, they know and love, experience. Thus, I gather that these academically-driven intergroup intervention strategies to advance knowledge, are why Luke perceived RAS to be so effective in combating the intergroup relations 
issues between White and non-White people. I understood Luke's proclamation of how extremely powerful it was to sit across from his peers, who looked him in his eyes, and hear their counter-stories to be his way of operating at the individual level to investigate how their nuanced lived experiences were influenced by social structures and vice versa. In like manner, Luke's responses forced me to brig attention to the role individual diversity development and other identity development theories has on student development. Particularly, as identity theories pertain to exposing people to identity groups other than their own, questioning ones' own family-driven belief systems, experiencing cognitive dissonance, becoming outraged by the newly discovered mistreatment of their own or other groups, and the desire to fight for social justice. All of which students are likely to experience when curricular interventions are crafted with transformational outcomes for its human ecological systems in mind. To achieve this end, requires intentionality towards the exploration of racial and social identities and the preferment of intergroup relations dialogue opportunities to mend the brokenness experienced across multiple microsystems and reinforced by macro and mesosystems. To validate said idea, the summation of Luke's response above addressed his appreciation for exploring the history of racial identities and the intergroup relations strategies that allowed him to discover and be enlightened by their stories of oppression, which affirm the need to advocate for healing and social justice. While I cannot infer that Luke's reaction to RAS can be generalized to massively represent all people of European descent-as doing so would perpetuate monolithic labeling - I do infer that it is an empirical indication higher education practitioners/educators should remain open to considering and exploring further, if they are truly interested in combating racial and social unrest through education reforms. Some practitioners/educators may be hesitant to shift towards combating racial/social identity unrest in such a direct way, out of fear for resistance. There are, however, creative pedagogical strategies-in addition to intergroup dialogue-that can be employed to create an academic atmosphere more conducive to widespread receptiveness among dominant groups who are likely to resist. One participant, Ida Campbell- 
Jones, who self-identified as a Black woman now in a doctoral program, shared some of her perceptions about RAS.

Ida Campbell-Jones gave insight into the ways she perceived RAS to help influenced behaviors to combat, advocate or face race and racism outside of the classroom by describing the nature of the intergroup relations outside of class with her White peers and their actions. She remarked:

...I think because we were able to engage with the material in such a personal way, it definitely struck conversations with, I know I have personal relationships, with some of the students outside the class. And I know that they talked about the class to their friends, to their roommates to their family members, and it was a good way for us to both like engage with the material but also like share what we learn outside of the classroom and a really easy, an easy way. I think because we enjoyed the class so much, it gave us a way to kind of, gave me a way to like reconsider what activism looks like and how music can be a form of both education and activism. Even just sharing material that I didn't think was easy to share was really, that was something that I learned from that class was just like, it's not hard to share material. It's not hard to share information that isn't as accessible to other people. And I think because a lot of people do, a lot of people in that class did share what we've learned outside of the class. It was a good way for people to 1 ) learn about the class and the work that Dr. Shonekan was doing and that Black studies was doing but 2) really see that this information in this curriculum is important.

After applying the Human Ecological Systems framework for analysis, I presume Ida's response to have described the networked relationships that ensued outside of the classroom itself. Essentially, she referenced the multiple microsystems of which she and her White peers were able to network with regarding the content acquired from RAS; namely, with friends, family, and roommates. All of which are 
their own microsystems, whereby, individuals find themselves influencing or being influenced by as a result of social interactions occurring. When applying a Critical race lens to these incidences, it is the nature of the conversations being held within the microsystems that is of importance; in this case, the dynamics of race in the American story. All the while, these networked experiences are eventualizing with aspirations to advance knowledge within various social systems. Thus, I construe the out of classroom exchanges to have been undergirded by the notion of transformation, which is the crux of Critical race research. Consequently, I presume the impetus for said Critical conversations to be grounded in the inclination to transform minds, attitudes, spaces, and pervasive systems of oppression. For the benefit of her own reflective thinking and reflexive praxis, Ida articulated her self-awareness and new-found level of comfort in sharing her lived experiences to advance knowledge. Analytically, I suppose, Ida realized her positionality as a Black woman and embraced the value of her voice and perspectives, in the realm of knowledge production through Mizzou's Black studies program. And additionally, inspiring people to see the Critical value in curricular opportunities like Race in the American Story. On the other hand, Ida explained her perceptions of the course's, instructional strategies, assignments, activities and projects as fulfilling its purpose to create learning opportunities around race and racism as:

...each professor had the same curriculum but had their own interest area [Disciplinary expertise] to which it was taught. And I took the class with Dr. Shonekan, her focus is ethnomusicology, so she focuses on music and I really do think that that was a great way to reach the students that were in my class, because it's very relatable. So, we were able to really kind of tailor the class to common interest. So, I think by doing that, it was really it was kind of easy for all of us to relate to the material because a few of our assignments were related to music specifically. And because almost everybody enjoys music, I think that it was really good for... both of us, like the material that she's teaching us but also to like, relate it back to her. 
The important takeaway here is the interdisciplinary pedagogical approach to ignite interest and feasibility of students engaging with materials and activities that yield positive intergroup learning outcomes. Explicitly, music was utilized in an academic manner to mitigate the existing or potential discontinuities within the intergroup relations of students from varying identities, because of its perceived ability to induce a sense of universal stimulation across multiple identities. Thus, music and intergroup dialogue appear to be suitable didactic strategies to mediate the historical complexities of race and racism in America. To underscore Ida's favorable perception of RAS's incorporation of music, Dr. Shonekan shared what she perceived-as facultyto be the most valuable critical curricular inclusions as:

Oh, gosh, I think Ida B. wells. Lynching in its Many Phases, I believe it's called, was critical. You know...I can't remember who recommended that piece out of the five of us. I think that piece even now, given what we've gone through this summer, you know, with Ahmad Arbury, with Briana Taylor and with George Floyd, you know, lynching from Ida B wells in Haiti in the late 1800 s and then we look at that document in 2020, It's chilling, you know. So, that's a really, really important one that really helps us pull that thread all the way to our current moment...and I think that the music part-of course I'm going to plug that-was an important, like almost chance to get away from the sort of deep traumatic readings that we were going through and give students a chance to kind of breathe and get that breath back, you know, and just get back into the rhythm of life and then go back in.

Anon, Dr. Shonekan alluded to the scholarly work of Ida B. Wells as a valuable contribution to RAS because of its ability to undergird the notion of a Permanence of Racism in American history. She quickly likened the historical manifestations of lynching to the pervasiveness of America's modern-day lynching of Ahmad Arbury, Briana Taylor and George Floyd, as a way to accentuate the horrendous intergroup relations between Black and White people that continue to impede efforts to improve notions of idle White supremacy. Simultaneously, she recognized the calming role her discipline in music had on students' ability 
to balance their frustrations and discoveries of America's very tumultuous and fatal history with racism. Reflecting upon the deeply traumatic content studied in RAS, knowing if anything aided or impeded Dr. Shonekan and her colleagues' implementation of this undoubtably pointed course would be helpful.

Professor Shonekan described her perceptions of how she and her colleagues were able to successfully move towards combating the discontinuities in racial intergroup relations, by first drawing from the perceptions of Citizenship@Mizzou that gave way to Race and the American Story. Because the Concerned Student 1950 group listed the creation and implementation of a required racial awareness and inclusion curriculum as one of its eight demands, it was important to inquire with Dr. Shonekan if that aided her team's efforts. She stated:

Definitely aided the fact that the students demanded that were not trying to see anything that was done halfway; they didn't want an online thing. And so that made the case for us to make it face-to-face. We couldn't meet one-on-one, clearly or even have small classes, small groups; we had to have very large gatherings of new students [Citizensship@Mizzou]. So, it really definitely aided the creation, the development and the implementation of Ctizenship@Mizzou. And I think that demand also helped to reinvigorate our creation of Race and the American story because we knew that as much as we had done with Citizenship@Mizzou, it wasn't enough, you know, it was still two hours. So, I know I was hearing shady comments from some folks I talked to. I totally get it, you know that "how can you do this in two hours?" So, we knew we'd done better than just an online thing, but we knew that we hadn't completely knocked it out of the park, you know. And so, creating Race and the American Story for us was our way of, of extending that in a pedagogical way that allowed us to give students more. 
Professor Shonekan perceived the Concerned Student 1950's demand for a required racial and inclusion curriculum to have aided the creation of both Citizenship@Mizzou and Race and the American Story. Thus, the analysis suggests that her perspective as Mizzou official's attention to the treatment of vulnerable voices and presentations, which also speaks to the recognition of how marginalized people were positioned in a position of power for the advancement of racial awareness and inclusion knowledge. Amid their propagation for curricular transformation are variables of time, space and Interest Convergence. To be clear, these findings indicate that Mizzou's institutional response to marginalized students' demands took form due to the time and spaces for which students protested under national media attention. Black and other marginalized student identities bask in experiences and systems of oppression daily. However, it was the moments of this case (time) and the space being occupied, namely, the quadrangle and Mizzou's football program, that propelled the institution's interest to converge and allow the space for episodic change to ensue. Mizzou's leadership stood in support of curricular interventions demanded by students and led by faculty, the roll-out of the first treatment (Citizenship@Mizzou) was careful not to use language and pedagogical strategies that might incite widespread resistance from White constituents of Mizzou. Constituents of whom may have been made to feel uncomfortable being required to sharply address the discontinuities in race and racism at Mizzou or in America; analytically said strategy is a Critique on Liberalism, which is why RAS had to delve deeper into the dynamics of race relations throughout American history. With this in mind, Dr. Stephen Graves contributed additional insights into the crux of RAS's curricular content; of which, is important for us to ascertain in our objective to understand the story of Mizzou's faculty-led response towards combating racial unrest.

To achieve the aforementioned end, Professor Graves expands our understanding of RAS's strategies to combat and reform campus unrest by sharing a wealth of information, which allowed me to analyze the data through the lens of his perspective. A significant amount of data from Dr. Graves was used in this report because of the thick and rich descriptive nature of his perspectives germane to the study. It 
was necessary to maintain the value and richness in his contributions by including his original voice and language used and to expand our understanding of how a faculty member philosophizes around racefocused curriculum. He expounded upon his earlier remarks above about following the story of race in American over time through the present by explaining:

So, it was important to be able to show and highlight that evolution, not just from a book, obviously, we're talking about Black feminist readings, and women's and feminist reading as well. And doing a visual of women at the same time. So, it really kind of felt real. We want to just tell as many of those stories as possible to paint the fullest picture possible of what race in America looks like. And I think that we were just trying to be as accurate as possible. We really wanted to just to provide the best mirror that we could possibly think of, to tell this racial story where in so many other instances and in so many other courses they only present a partial history. And we felt like there was nowhere to really tell a story of race in America, we wanted to hold up the biggest mirror that we could possibly that we could with the many different opinions and ideas, intellectually as well. And so, telling that story was important.

The faculty's value in examining the intersections of race and gender was extrapolated by including the scholarly works and stories of Black women discussing the role of Black women during feminist movements by reading both Black feminists and women's feminist works. Said strategy would allow students to understand the experiential differences and oppressions Black women faced in comparison to White women, in the fight for women's rights. Dr. Graves and his peers wanted to expose the truth about the story of race in American history, because of the pervasive exclusions of race-focused content amid our education system, which implies a maintenance of Whiteness as Property. For example, some people may assume that both Black and White women were unified in their attempts to obtain rights for women; failing to consider the ways women who also identify as Black were marginalized in a movement fighting to 
bestow rights to only one part of their identity while continually oppressing their Black identity. Graves perceived RAS to fulfill an academic void in an education system that has refused to grapple with America's racial history and listen to the multiple voices, experiences and counter-stories of Black people and their intersecting identities. Graves emphasized the notion of a White dominated education system that excludes Black thought by describing the intellectual philosophies absent from mainstream curriculum:

... again, some issues of Black nationalism, some of the other stories that aren't familiar that haven't really ever been told about Black power, Black nationalism, or Black separatism, and everything else that say "Black people should have their own thing, selfdetermination, and everyone's ideas," these ideas for some people are not just radical, but some are unheard of. And some of these students never even heard of these ideas before.

Professor Graves named several Black ideological thought movements that are often not heard or explored intellectually in the historical context of America's history with race. Consequently, the analysis suggests that Graves contended that if the education system continually excludes the counter-stories of Black voice, it will force people to apply their own definitions and perceptions about the status, aspirations and racial plight of Black people today. In fact, he explains his perceptions as follows:

...there's a lot of people growing up thinking that when you get to Emancipation Proclamation and get on to M.L.K. [Dr. Martin Luther King Jr.] that African Americans are just looking to be part of the American project and we're looking for inclusion. Well, there's a large chunk of people and a high percentage African Americans at a point in history who said, "this country ain't for us. And we really don't want any part of the sale. So, this just give us our freedom and justice and let us go do our own thing. And we're not trying to be incorporated. And we're not trying to be involved in white America, And America is in some cases is the problem. The problem is sometimes with the race in 
America's story is White people, right? They are the problem with racial injustice." Nowhere else in Missouri, are these students going to hear that message or get that perspective. They just think everyone's trying to be M.L.K. and just Kumbaya this thing. Well, no, there's actually opinion out there that says, "Black people don't need this shit. And we're not interested. And we can do our own thing." And so introducing those ideas and opinions is heavily important. So, that was a contribution that that was highly necessary and highly important when you're trying to get a full picture that not all African Americans are just trying to walk hand-in-hand and Kumbaya this thing out and believe that we're going to turn the other cheek, and that love is the answer to all of our problems.

Professor Graves elucidated the preconceived notions that some dominate racial groups hold, which undermine the diversity of thought and ideologies of African American people; perpetuating a monolithic view of how Black people think and experience life as an American. By failing to operate at the individual level to investigate the nuanced lived experiences and perspectives of Black people or pay attention to the variation of their historically held beliefs, it is likely that people will assume that all Black people desire to nurture intergroup relations and be engaged in a fellowship of love with their White counterparts. Such thinking is dismissive of the pervasiveness of racism and may subsequently infer an eradication of structural oppression causing Black people to only be desirous of acceptance, approval and validation from their White counterparts, because - their eyes - racism has ceased since the Emancipation Proclamation. Dr. Graves' perspectives supports the examination of Black voices who unapologetically did/do not wish to live harmoniously at the will of White American norms but who only demand equity and justice to establish their own way; unleashed from the domineering control over their right to enjoyment, disposition and use of space in America. In other words, to combat the unrest within intergroup relations at Mizzou, Graves adamantly perceived the incorporation of diverse perspectives as instrumental in 
helping students understand the full picture of America's treatment of race and why some Black people feel different about the ways in which they wish to exist and interact or not with their White counterparts. When some Black people are hesitant to share their knowledge or experiences to advance knowledge or when some Black people do not wish to reply upon White acceptance or align with White normalcy to live harmoniously, it can be better understood through the academic discovery and examination of multiple viewpoints. In fact, Dr. Graves framed it by saying the following:

...Man, some of us are a little more realistic than that. And we're not interested in all that nonsense. And we're trying to get what we're deserving of and get justice and get what we believe that we're do and whether we do that with White people or not it's not our issue. So, those contributions, I think were just as important if not more important than the basics. Yes, it's important to have the very foundation documents and everything else from the founding fathers, but different and diverse opinions about this country, even Frederick Douglass and the Fourth of July. That's always been a good discussion that students have had about how Black people celebrate the Fourth July. We don't really celebrate the Fourth of July, it ain't really freedom for us, we don't do fireworks. We don't do American flags. We don't wear you know, flag t-shirts and nothing like that or are really interested in that whole thing. And so how these two groups just have complete differing opinions of the same country was something that we really want to bring out. In the curriculum and the way that it was dealt with, so, of course, it's you know, it's timeline centric. So, the course is broken down into timelines from, you know, pre-revolutionary, and to the revolution era to, you know, civil war to reconstruction, to civil rights into the modern era, we get into Obama and everything else, it towards the end. So, it's meant to be broke down, you know, chronological order and some kind of a timeline. But the emphasis on that, you know, diverse opinions and the differing opinions, especially you start getting 
the, you know, the black readings was is huge, and probably what I would argue are the most important readings in the series.

Dr. Graves highlights one viewpoint that is primarily concerned with receiving justice and equity over the centrality of harmonious intergroup relationships. He uses this idea to reinforce the importance of students being exposed to the diversity of perspectives about race relations; just as much, if not more that the foundational American documents. Additionally, the professor referenced the ongoing discourse about the role of the Fourth of July in the lives of Black people and how, a Critical race analysis points to the idea that it is not a holiday that symbolizes freedom for peoples of African descent. In the faculty's attempt to work towards fighting for improved intergroup relations, his responses indicate that they saw value in examining the chronology of critical moments in America's racial history, to gather up the diverse opinions and perspectives needed to progress those relations. Time was important in the learning process of RAS, as Dr. Graves articulated that their organizational course strategy was intentionally structured for students to explore the role of race and racism in a chronological order, to best follow the story of race in America. Doing so, would certainly require a high level of preparedness, comfort and personability to be effective at an attempt to elicit transformative outcomes.

Given the transformative nature of a race-focused curriculum, it is helpful to understand faculty and students' perceptions about instructor preparedness and their aptness to teach in this realm. Ida gave the following response:

So, I don't want to say that I'm biased but Dr. Shonekan is one of the best professors that I've ever had. I think because she is so passionate but also, she has the curriculum behind her passion and almost every class is like a passion project to her. And I think that adds to her preparedness. She's taught almost every kind of student and I think her ability to connect with students, to know how college students operate, but to also know how people 
are and how to adapt means she's very adaptive in every classroom. And I think with her background, both being in music and in Black Studies, it definitely gave our class a different kind of, it gave our class a different kind of habit experience. A lot of the class's [RAS] have professors that are dual department professors. So, I think that even in Dr. Steven Graves class, he's political science and Black studies, and Dr. April Langley's class, she's English and Black Studies. So, I think the professors that taught the classes brought their broad experience and knowledge, it allowed each different class to have a different experience. There was more than just Black studies. Even though Black Studies is enough, like you have the different experiences both with the professors and of all of the students in the class. Because they weren't just English majors in Dr. Langley's class, there weren't just political science majors, like we had journalism, we had I think maybe education. So, the passion behind the professors as well as they're good.

Ida's perception of her professor's aptness to guide students in RAS stems from an awareness of the intersections of Dr. Shonekan's identities as a Black woman, ethnomusicologist, and Black studies scholar who possesses an innate passion to perform the work of a Critical Race scholar to advance knowledge. Her mention of Dr. Shonekan's ability to engage students, in this work, from all backgrounds is a testament to the professor's value as a person who is in a position to produce knowledge in an intergroup setting. The CRT/I framework leads us to consider the ways, in which, people from traditionally marginalized communities are situated in notions of power and knowledge production. Ida's comments continued along these lines as she began to describe the vitality of the inclusion of backgrounds and experiences brought to the classroom by RAS's other faculty instructors, of which, also hold intersecting racial and scholarly identities. As she reported, the interdisciplinary approach to guide RAS's mission was largely successful as a result the combined variation of experiences of both faculty and students, who were racially and disciplinarily diverse. Ida's admiration for passionate professors, validates Jacob Sommerscale's earlier 
mention of the important role passion plays in carryout this Critical work; this serves as another point of reflection for practitioners/educators engaging in this work. In response to a similar inquiry around teacher preparedness and whether RAS was considered by him as surface level, intermediate, or in-depth, Luke Davis shared specific examples of the course's content that informed his perspective about the courses instructional strategies that proved why he felt as though his professor what thoroughly prepared to teach in the realm of race. He shared:

Well, so starting off with the instruction method, um, I've said this before, but Dr. Shonekan was a good guide; she was exactly the right kind of person...she has a deep and more understanding as a Black woman and as a professor of Black Studies [Inaudible] she just wants us to be understanding of these issues and how it relates not only to our history but to work ethic as well. As for the way that she structured the class, I think she has a really good style and is really personable, she's really about the students and students know that she cares about you [them] and wants you to learn in that environment. And she's careful about not ostracizing people. And also making sure that there isn't going to be any kind of racism or homophobia or any or any of that kind of behavior. And not only that, she goes out of her way to make sure that all of her, all of our students feel safe. As for the assignments, they were great assignments. We were reading Phyllis Wheatly, we read Frederick Douglas and Washington, W.E. B. Dubois and all these other fantastic Black writers and historians and activists and also Latinx, activists and writers and, and even White writers and White historical figures. It was it was by no means surface level and even as someone who had dug a little bit into that scholarly literature and into Black authors and writers, she exposed us to so much that I never would have found even with people that I had read before, right, you know. And like Douglas, with what is July Fourth to the slave ["What to the Slave is the Fourth of July"], that's typically one that people read in classes of that nature 
but probably brought about four or five or six even other works that were famous at the time but may have been a little more lost. And you know, that I had never heard of that before from Douglas and she also another thing that was particularly powerful was we dove into a Sojourner Truth speech that has been revised over the course of history, and has been, I think just need to kind of evolve about women, about Black women's Suffrage rights and over the course of history it has been edited to make her sound like she's more from the south when she was raised in like Pennsylvania. And we dove into that right, we read this one to an even further level and historians argue that this is not what Sojourner Truth even sounded like and so not only did we examine primary sources that I hadn't been exposed to before but we dug into how those might have been revised and how, you know, this redemption after the Civil War, this resurgence of, of the KKK and of White supremacy had revived so much of our history. And so yeah, it was, it was a deep dive into the historical and contemporary, so it was definitely, it was definitely anything but surface level.

Immediately, Luke referenced Dr. Shonekan's intersecting identities as a Black woman and professor of Black studies, much like Ida Campbell-Jones did in the previous findings above. As we reflect on the significance of her identities, we should consider how her Blackness intersects with her identity as a woman and furthermore, how her identity as a professor of Black studies intersect with her being a Black woman. All areas of traditional marginalization but, as earlier stated, it is how Professor Shonekan has been positioned to aid in the process of creating new meaning and producing new knowledge for those of whom she instructs. Luke recognized the value in Dr. Shonekan's intersecting identities and how they advanced the process of coming to understand the nuanced lived experiences from those who have often not had a formal platform to share their counter-stories. He, like Ida Campbell-Jones, spoke to Shonekan's personable nature and ability to make students feel welcomed in her classroom; an example of another microsystem, in which, students network with other individuals different than them. Along these lines, Luke-a White male 
himself-expressed a value in his professor not making students feel ostracized, perhaps, for their White identity, while navigating the learning space. Space, is referenced in this instance to highlight the importance of creating a safe and welcoming learning environment-within the human ecological system-where students do not feel marginalized and are shielded from incidents of intentional racism and homophobia. Luke's thickly described examples of diverse historical course content underscore the faculty's reports about exposing students to a wide-range of diverse perspectives, within historical context, to examine the story of race throughout American history. Of which, we know, the CRT/I framework encourages us to do in order to understand the ways race has determined how people-considered to be inferior to White people-have experienced continual oppression through America's structurally-designed inequitable systems. He validates earlier perceptions reported in this chapter about the newness of information, traditionally excluded and overshadowed by Whiteness, that many students have never be extended the rights to learn in a system where intellectual property is controlled by people who value White normalcy as the American standard. Specifically, Luke described an example of a counter-story discovery as his class explored and uncovered the truth about Sojourner Truth's counterfactual dialect that made her appear to people as a southerner rather than from Pennsylvania. It appears this was done to have her be regarded as an uneducated southerner, as an attempt to discredit her intellect, activism for women's rights and as an abolitionist to slavery. Luke pointed out that this type of deep dive, along with discussions about various movements over time, are a display of how stories have been told inaccurately, counter to the true stories of people from oppressed groups. Thus, all people deserve the right to said intellectual property that includes the counter-stories of the oppressed. On the other hand, the responses were collected from the faculty who also shared their perceptions about how to ensure teacher preparedness. Given the sensitive nature of the course content, participants were asked to, "talk about the qualifications and resources to sort of gauge or ensure teacher preparedness for instructing or facilitating either or both Citizenship@Mizzou and Race in the American story." 
Although this section is primarily dedicated to how Race and the American Story was used as an educational tool to combat the discontinuities of intergroup relations, particularly around race, Dr. Shonekan's brief mention of Citizenship@Mizzou is referenced in this section. Specifically, because in her response to the inquires around ensuring teacher preparedness, she explained the slight difference in how this was done with both interventions. She stated:

I think we were very careful about who we worked with. Adam and I just met with our new colleague who will be coming to Mizzou, and we'll be teaching the course this fall [Fall 2020]. We just kind of talk with people, it's not an interview or anything, we just want to see what they are, what their training is and how their own reflections are on race in the American story. So, for the first Race and the American story all the faculty who teach are PhDs in one discipline or the other. I think at some point that course can definitely be taught by grad students as well. But it does need some degree of maturity, to be able to moderate discussions that are sometimes very difficult. If you're a big fan of Thomas Jefferson, and we all read what he said about Black people in notes on the state of Virginia, your feelings are going to get hurt, you know, you're going to learn some things about your that you didn't know and that you now see other students react to viscerally. And so whoever's at the helm, whoever's teaching that course has to know how to help the students negotiate around those epiphanies and come out at the other end in a way that's constructive and productive, and generative, most importantly. And so, that's how that's done recently in American story. Citizenship@Mizzou, because I had I worked with students, these are students I knew very well, that again, very personal right? So, I knew these students, they've been in classes with me. I knew they were wonderful musicians. But I also knew where their heart was in terms of their love of Mizzou and their push towards social justice. And then, of course, the faculty as well. I knew the faculty members very well. One of them was a professor of medicine who 
I had heard talk a lot about growing up, he's Japanese American, growing up in an internment camp you know, so he had a different entry point to thinking about citizenship, that was compelling because not a lot of people had lived in internment camps, but most of us have heard about them. So, we could take that story and apply it to what we will going all the way through until we get at Mizzou. So, I kind of was really careful about who I chose to work with; who also could carry a story, tell a good story right? In two hours, you really need engagement, you know.

The passage highlights the Critical approach curriculum developers must take during the selection process before onboarding new faculty into the role of instructor for Race and the American Story. Dr. Shonekan recalled she and her colleague's process of meeting with a colleague to ascertain their ideological perspectives about race and its role in American history. This appears to be a part of the process where the course developer's took a moment to analyze, with a Critical race lens, the new faculty's disciplinary background driving their philosophy, their level of comfort, and approach to advancing knowledge in the realm of race. Dr. Shonekan hypothetically perceived that new faculty-who admire Thomas Jeffersonhoping to teach RAS might be emotionally hurt when they discover the Critical race examination of Thomas Jefferson's historical notes. In this case, we must consider how said response might undermine the transformative nature of the course, if they reject the notion of unraveling the favorable view of Thomas Jefferson maintained by some today. In other instances, it may cause the new faculty instructors to feel uncomfortable and ill-equipped to facilitate an intergroup relations dialogue with students, of which is supposed to be exposing students to diverse voices and perspectives. If Dr. Shonekan and Dr. Seagrave were/are not careful and fail to examine how potential new faculty will treat the voices and perspectives of those underserved, it may negate RAS's attempt to combat the discontinuities in race and racism amongst White and Black people historically. Analysis suggests that Dr. Shonekan operated at the individual level to investigate the nuanced lived experience of a Japanese professor who grew up in an internment camp, by 
including his perspectives and experiential knowledge in Citizenship@Mizzou; a way to allow someone from a traditionally marginalized group to be positioned in knowledge production and counter-storytelling, on an intergroup public platform. Likewise, Dr. Seagrave also reported his perceptions about resources to ensure teacher preparedness. He described his perspectives by stating:

That's a good question. Because as you said, these are really, difficult, controversial topics that we're covering. And, it's difficult because the faculty, right, we have different backgrounds and perspectives and that's so it's done well. So, one thing is that the core syllabus and the core readings keep us grounded and on the same page to a certain extent regardless of the backgrounds of faculty. I think that's pretty key that they were focused on primary source readings from American history. And that's what drives the course by and large. So, I think that helps. ...I think we, as we grow, I think that is something that we will do more of, so just this past year, we had our first faculty summit; a meeting of all the faculty talking about issues in teaching, how we approach things, problems we encounter, things like that. And I think we're going to do more of that in the future. And I think that's needed. So, faculty meetings across universities, to share their thoughts, concerns, etc. Tips, you know, helpful advice on teaching these topics. I think we need more of that. So, we're just starting to build that out really, actually now as we grow. Because it is really difficult. I think, it poses all sorts of challenges, trying to lead discussions, especially if you know what we're trying to do is bring together students who have very different opinions and backgrounds. It's not easy. It's something that that we will be doing more of, and I think it's very needed.

While Dr. Seagrave recognized the benefit of having faculty from diverse backgrounds, each with their own unique lived experiences to teach RAS, he also stated that the syllabus and primary source readings of American history grounded the course and helped faculty maintain reasonable consistency across all five sections of RAS. Consistency within the curriculum would of course safeguard the overall transformative 
mission and purpose from alternative competing or combative ideologies. To further protect the environment and student learning experience from being undermined by unbridled resistance to the transformation of intergroup relations, Seagrave expressed value in their developing faculty summit, whereby, each professor can be reflexive about their experiences teaching RAS and what they discovered about themselves or strategies that might need to evolve in order to improve the learning experience. As a reminder, reflexivity creates the space for constant Critical self-reflection and awareness. The faculty summit was the space where these reflections and discoveries could be shared and discussed for added levels of awareness about recent or future faculty experiences, that could potentially aid or impede the curricular mission. Additionally, the coming together of faculty allows them to discuss the intersections of their own social categories or their students and how they interact or co-constitute; another component of the CRT/I analytical framework to consider. Along these lines, Dr. Graves' robust responses led to the discovery discover of the ways in which White and Black professors may differ in their attempt to teach examining the history of race in America.

Dr. Graves responded to the inquiry to discover what he perceived to be important advice to create the most ideal experience for faculty at other institutions hoping to teach a course like RAS. The findings provide a wealth of insights to advance our knowledge on how White faculty and Black faculty must be mindful of their Whiteness and privilege and history of being marginalized intersects with their positionality in knowledge production, respectively. While his response somewhat overlapped with the questions about teacher preparedness, the conclusion is that it would be best to briefly share the idealistic gist of his comments here; foreshadowing what will be detailed in a later section in this chapter on organizational change and institutional reform efforts. Professor Graves' initial response giving advice to faculty hoping to teach RAS at other institutions, revealed very strong feelings towards White professors' need to be adequately prepared prior to deciding to teach such a course. It was also very clear that he is an advocate for young and passionate professors of color to teach RAS, because they will offer a unique perspective and 
ability to challenge and push students in course discussions that might otherwise be difficult to achieve with non-Black faculty, who do not share similar experiences as their Black colleagues. It would be helpful to understand how he, as one of the course's developers, would work to safeguard the mission and ensure that any faculty member who attempts to teach RAS is prepared to do so. He proclaimed:

Man, it's a little bit different. One, to be perfectly honest, it's not my job to teach White people about how to teach Black people things. I'm not interested, good if you're going to try to ever go to sign up to teach, teach a class like that. Hell, let alone take a class like that, then it's your job and your responsibility as a professor and as a professional to take it upon yourself to learn those things. This is not my job to babysit White professionals through Black history, or Black politics in their in their own courses. Think about that before you decide to teach a course like that and what you can add to. Now if they ask questions, and if they come to me, that's a different story. And I'll point them in the direction of reading. But mostly, what I like to do is just have a conversation with them. I'm not going to tell them what to say. I'm not going to tell them what to naturally read but I will just have a conversation with them about the readings, or "what do you think about when Malcolm says this" or whatever else it is. And that way, if they can know where I'm coming from, maybe they can take that to their class, but I'm not going to babysit them or tell them exactly what to say, and what to cover or anything else, whatsoever at all.

Graves's high energy and passion during the interview exuded as he proclaimed his perceptions about his role to ensure teacher preparedness for those in line to teach RAS. Particularly, he channels an opinion that is intriguing to the work of a CRT/I researcher, as he discussed his positionality as a Black person in the role of knowledge production for White colleagues. To be clear, Dr. Graves strongly expressed his oppositionin this context-to being positioned as a "babysitter" or teacher for White colleagues; he thinks it is good for White faculty to sign up for such a course but feels that it is not his responsibility, as a Black person, to 
help White colleagues through Black history and Black politics but rather their responsibility to do the necessary research to prepare themselves. Essentially, it seems that he was urging White faculty seeking to teach a course like RAS, to think about their own identity and ability to be prepared to advance knowledge around race and racism, Black history and Black political thought. Given the historical and continuing dominance of Whiteness as Property within the American education system, it is infered that Graves perceived that the possession of in-depth knowledge about Black history and politics for White faculty-in general-would require an intentional journey towards discovering and understanding varied Black experiences of Americans over time. By and large, the American education system does not automatically include race-focused curriculum to highlight the counter-stories, opposite of White-driven narratives, it is not commonplace or natural for some White faculty to attain such knowledge unless they make it a point to educate themselves in a, presumably, unfamiliar discipline. Professor Graves did, however, share that he saw his role as more of a guider for White faculty, who is willing to guide colleagues towards helpful resources and share opinions gleaned from readings that might contribute to their meaning-making and course direction. Given said reality, Dr. Graves worked to counter the notion of Whiteness as Property and the exclusions of race-focused material by doing his best to raise awareness about America's history and treatment of race. He explained how he ensures his own preparedness to teach RAS by stating:

What I am going to do is, in my section of the classroom... I'm just going to go all out and just cover the hell out of that topic. And I'm going to give it all perspective, all sides, I'm going to be a little controversial and a little rebellious. And I'm going to make sure that the emphasis on the Afro-centric and the Black parts of it are heavily extreme, and make sure that students get all that they can. And that way when they leave my classroom and the next time they see that stuff being covered in a White professor's classroom, they can harken back to my class and be like, "wait a minute, this shit ain't the same, that ain't what Dr. Graves said, and I've seen some readings before that ain't covered like that" and say "wait 
a minute, professor that ain't what happened;" that's my thing. I think that the students in another professor's classroom saying, "Man that shit ain't true" goes a lot further than me pulling a professor to the side in the hall or at lunch saying, "what do you think about having them read Malcolm X"... that's one thing for sure, if they get a student or get multiple students in their class, raise their hand and somewhere else I'm saying, "it didn't happen that way," or "the Black people have been getting a raw deal for a lot longer than that" and this and that. Now, the professor is going to have to realize that there's information out there being shared that they're not comfortable with and that they don't know about, that the students know about. And if you can show to the student that you have as much information at least that they do, then that professor is going to be in trouble. You know, teaching wise, your teaching evaluation wise if the student has more information than they do. There's always going to be special readings where a student has more information for sure. But on a factual basis, if you try to breeze over something that's factually incorrect that the student has been introduced to, that's going to definitely be a problem.

Analytically, Dr. Graves reinforces his insistence on helping his students become comfortable with paying attention to the historical power dynamics in American structures, how they have been and need to be challenged today. In doing so, he spoke of delving deeply into the Afro-centric parts of the curriculum, as a way to center race, culture and identity as the focal lens, for which to examine how they have been treated, excluded, misinterpreted or defined by White-controlled ideologies. He does so through the exposure to multiple perspectives and dialogues that prepare students to enter other classroom spaces on campus with-what I infer to be-a Critical race lens as they encounter material from White professors. Dr. Graves clearly stated that he expected his students to be able to call out the inconsistencies, false or incomplete narratives about the experiences of Black people throughout history in their other classes and share their knowledge from his course or perhaps how they have been taught to process information in general. 
Analytically, we can tie Graves' perceptions and practices to the ways in which all people can challenge power in the classroom and position themselves to advance knowledge in the realm of race and intergroup relations; ultimately pushing back, yet again, on the notion of Whiteness as Property. Consequently, he argued that White faculty who are not prepared to deal with, know of or address their stance on aspects of Black history and Black ideological thought, will find themselves in an incommodious predicament in the classroom and uncomplimentary course evaluations. As Professor Graves continued sharing his perspectives, on White professors who may feel ill-equipped but have a desire to teach RAS or a course like it, by doubling down on it being "...their onus to divulge themselves into the material, enter the readings and seek outside opinions." Therefore, if they do not properly prepare themselves according to what he suggests, he expressed:

If not, they better come to a class, get in line and watch for themselves and get from other Black professors how they go about these things and figured out how they can implement it in their own classrooms. Black people in this day are not here to try to train and hold White people's hands through racial history and racial knowledge and everything else it is. Because they've had an opportunity for hundreds of years to do this thing and they refused to do so. And now we're going to take up the mantle and do it our way. Then you guys just stand over there to the side and watch and maybe learn something and maybe you can pick it up and take it to your classroom, but it's not our job to train White people on Black education.

This dilemma forces us to pause and reflect upon the multi-layered and varied experiences and ideological perspectives of Black people and how they see themselves positioned in advancing knowledge. But what was discovered after looking more closely, was that not only do not all Black people see themselves as responsible for advancing knowledge about Black history and ideological thought, not all Black people who do strive to advance knowledge are willing to do it for people of all identities. In other words, for some Black people, race matters when it comes to who they feel obligated or innately driven to produce and advance 
knowledge with. Some Black people may only feel responsible for advancing knowledge within their own racial and social identity groups. There may be any number of reasons as to why this may be the case, including Racial Battle Fatigue, but assuming that all Black people-faculty or otherwise-desire to or perceive themselves to be responsible for educating their White counterparts can be a huge blunder in judgement. Dr. Graves's perspectives forced further analysis regarding the role of traditionally marginalized people in notions of power and knowledge production. For Dr. Graves, it seemed that while he is a passionate and intellectually-driven scholar who is dedicated to teaching, he accepts the responsibility for formally teaching students from all backgrounds rather than his White faculty counterparts who should own their own educational journey. He sees them as capable of dedicating themselves to Black history and ideology if they truly desire to most effectively and accurately advance knowledge in this realm, as they have full access to the same resources he used to prepare himself. Considering the various levels of preparedness to truly move towards curricular reform central to race and intergroup relations, we should be reminded of the role that faculty of all intersecting identities play in the advancement of cultural knowledge for all students. Thus, it is intriguing to understand the perceptions of how often all students should or will be exposed to content on race, racism, social justice, intersectionality etc., to experience curricular reform, that could potentially lead to improved intergroup relations. With this in mind, knowing the perceptions of faculty and staff on whether or not Citizenship@Mizzou and Race in the American Story should be optional or required was vital to the study.

\section{Required or Optional Racial Awareness Curriculum to Elicit Institutional Reform}

During each of the interviews, all participants expressed strong opinions about whether Mizzou's curricular interventions following the protests should be required or optional. As a reminder, the Concerned Student 1950 group demanded a required racial awareness and Inclusion curriculum be implemented for all 
University of Missouri constituents. While the initial institutional response made Citizenship@Mizzou a requirement, it was only required for freshmen and transfer students. Likewise, the training course was not sharply focused on racial awareness as much as CS1950 may have imagined, according to the participant findings reported in this chapter. Therefore, the students did not receive the full benefit of their demand being implemented in its entirety. However, as the small group of faculty members gathered by Drs. Shonekan and Seagrave led the creation of a more deliberate race-focused institutional reform effort to supplement what C@M lacked, it was an optional course offering. I was also necessary to know if a required course designation was perceived to influenced or deter students' desire to learn and engage in the course. What emerged from the data is worthwhile for higher education practitioners/educators to consider when attempting to evaluate or implement curricular reforms where race, racism, identity, social justice, as they are central to the learning outcomes. This section presents the findings regarding the participants' perceptions about the notion of required vs. optional approaches to institutional curricular reform.

\section{Citizenship@Mizzou}

First, a student facilitator and musician in C@M's Talking Drum band, Jacob, shared the following:

I know, the University also tried to implement a Citizenship course for incoming freshmen, and it was meant to be a kind of a large-scale requirement course to kind of introduce students to, you know, racial issues and stuff on campus. But it was a required citizenship course for incoming freshmen that they were all required to take, which had a little bit of pushback with that, I think anytime you try to force kids to take a class that they may not be interested in or just force them to take any class period, there's going to be this kind of feeling of being infringed upon and they're not going to be as active in it. And with it, with large classes for Citizenship@Mizzou courses, for a lot of incoming freshmen, that kind of discussion-lecture, you know, part that really doesn't get to happen. 
Jacob first recognized the likely behavior and attitudes of his peers, as some pushed back on the idea of a required course in general; perhaps, feeling forced and infringed upon. What is important to note was Jacob's mention of what he felt did not get to happen; the lectures that are supplemented with more deep discussion. According to Jacob, given the large size and limited time of Citizenship@Mizzou, it appears to have made it difficult to invoke active participants, who were not initially interested in diversity training, without smaller dialogue-driven learning environments. Jacob was asked to be reflective about what he would do to improve the course, in his response, he described how he perceived Citizenship@Mizzou and Race and the American Story as needing to both be required. Jacob explained:

I probably would have done that [Require Race and the American Story] in conjunction with Citizenship [C@Mizzou] while making Citizenship a little bit more explicit. And making it an ongoing thing. At times it felt sort of performative by the university, it was like, you kind of make people do one thing and then it's over and then you kind of engage with problems and mild racism talk on one Sunday morning, and then you don't have to mess with it for the rest of your time at Mizzou. You don't even have to think about it if you're of a certain demographic or something. So, I think making those things mandatory [is good]. Jacob candidly asserted his desire for Citizenship@Mizzou to be more explicitly about race. This is consistent with other findings reported earlier from participants who remarked about some student and faculty's unfulfilled satisfaction and opinions that there needed to be more than what C@M offered. He further stated he felt as though the university officials were being performative in their initial curricular reform response because people only had to mildly face the realities of racial unrest once, in a large setting and-if they wanted-never have to look back or discuss it again if they are a part of the dominant population on campus. Interest Convergence and the Permanence of Racism seemed to align with this response. Like other instances within this case and given the heavy negative national attention it received, requiring a diversity training course for freshmen and transfer students was a way to appease students and 
repair the damage of Mizzou's nationally known racial campus unrest. However, the analysis suggests Jacob’s comments to mean that by not requiring a more racially explicit Citizenship@Mizzou and ongoing diversity education/coursework, it fails to dismantle the notion of the pervasive racism and oppression upheld by those who lack interest in formally learning and discussing race and those practitioners/educators who fail to make ongoing progressive race-focused curriculum available. Jacob perceived universities in general, as being hindered to make such explicit institutional reforms because of what they stand to lose financially, align with interest convergence. Jacob explained:

The worry about these universities is that there're donors and the donors have their opinions about how the university should be run. And if those opinions aren't met they'll pull their funding and then what's the university gonna even do without funding? You know? So, it just always felt like they were trying to appease their conservative White donors by not really engaging and sort of just brushing it under the rug. So, I think very explicit support from the Chancellor would have been good. Very explicit support from the chancellor, regarding that concern student protests would have been helpful. Again, reforming the kind of required curriculum to be explicit and, and constant. I think that I would go so far as to say every year you have to take a course that has to do with race or something. The idea is that this thing doesn't go away just because it doesn't exist to some people or you donate some money to the George Floyd fund doesn't mean that the shits over and there are people who don't have the opportunity to not engage with this. Jacob, essentially, described how Interest Convergence works by framing the financial impediment that could result from university officials making structural and policy changes incongruent with the values and belief systems of their biggest donors. Thus, drastic and massive organizational change and institutional reform may come at a slow pace and be packaged in more subtle ways; ways of which, will not anger donors, other stakeholders and even the tuition-paying families of students who enrolled. But, 
unfortunately under analysis, that described method is a Critique of Liberalism and comes at the expense of an improved human ecological system and campus racial climate for those who are marginalized or demanding change. To undergird this analysis, Jacob pushed back on the Interest Convergence and Critique of Liberalism he described by proclaiming what he perceives the university's role is when deciding to require or make race-focused curriculum optional. Jacob proclaimed:

And I think that it's the responsibility of a learning institution to make people learn via some amount of discomfort, we don't censor the Roman history class talking about raping and pillaging and we don't remove that because it's too hurtful or something or it's too explicit. So making things clear that race and racism are an important part of our history and pretty much included in every course like, English, or some literature course. I guess it all boils down to the role of the university to make people grow. And growth usually happens via discomfort. So, making things mandatory and being very transparent and explicit about your support of students would have been good.

Jacob described what happens in settings where intergroup relations dialogue is used as a strategy for making new meaning and advancing knowledge when he mentioned the inevitable reality of discomfort felt by those engaging in Critical conversations. Analytically, the White Racial Consciousness model can be used to draw parallels to Jacob's assertions about learning through discomfort. For example, to some degree, discomfort and dissonance is likely to be felt as White people are presented with the realities of race dynamics, racism, and the impact of a dominant White status quo. Subsequently-as presented in Chapter Two-Row et al. (1994) stated that the way in which dissonance is resolved is the main cause for a change in racial attitudes, thus, the more significant the amount of dissonance one encounters, it determines the "type" of status they move into; either Unachieved White racial consciousness or achieved White racial consciousness. As White people wrestle with the historical legacies of racism and reflect upon how their Whiteness is situated within notions of being complicit in the Permanence in Racism or dismantling of it, one 
must make a decision to perpetuate or fight against inequitable systems. Jacob contends that it is our learning institutions' responsibility to create intergroup learning environments where the grim historical truth about race relations is presented despite potential discomfort, as a way to ensure students are afforded the opportunity to investigate the intersections of race and identity to capture the impact of American structures on traditionally marginalized groups that are often excluded from the curriculum. To this end, Jacob advocated for the inclusion of race and racism to be included in every course-in some waygiven their important role in history and not to exclude race-focused content that make people feel discomfort because we include the dark past of classical Roman History without a problem. Jacob was not alone in his belief that Citizenship@Mizzou should be required.

Zoe valued the idea of requiring Citizenship@Mizzou but recognized the drawbacks this approach would yield as a result of disinterested students failing to contribute or engage in conversation. During her interview she reported:

So, I think that this class is so essential to be taken by students especially like, like I came from Boulder, Colorado, which is a predominantly White area. And so, coming into a university that was, like, even on its own, just different from what I had known previously, I thought that having access to professors and students who shared their stories on that stage and provide a little or provided a little bit of insight in terms of their experience as a person of color or an LGBTQ or person. I thought it was really essential and really empowering because I am also a POC and then also an LGBT. So, it's really cool to kind of see allyship in that. But I do think that the fact that it was required I think it was important that it was required because it's important that people are in that conversation when maybe it wouldn't have been in their natural progressions to be in those conversations. But because people who are required to be there, obviously people who didn't want to engage in the conversation had to be there as well so, there were people who didn't contribute positive 
things to that conversation. But it's important that everybody bear witness to those comments.

Zoe used her demographic background and intersecting identities to rationalize the importance of students being required to take a training course like C@M. She argued that it was important for her as a person of color and a member of the LGBT community, to hear the sharing of lived experiences from other people of color and LGBT identified individuals to capture the variation in perspectives and to witness a human ecological environment where allyship is encouraged. She believed that while some individuals may not have naturally opted to or been exposed to a course of this magnitude, it was good for them to be in the learning space where meaning is being made and knew knowledge around race and identity is being produced. On the other hand, Zoe perceived some of her peers as disinterested and unwilling to contribute positive aspects to the conversations held in the training. Analytically, it appeared that both time and space played a role in achieving successful learning outcomes. Despite the disinterest of her peers, she believed it was more beneficial for those individuals to be in the space to listen to the voices of those from marginalized communities as they shared their nuanced lived experiences. Likewise, it seems that the limited time for each C@M session-as indicated in the responses of other participants-hindered the magnitude of effectiveness, particularly on those who were apathetic from the onset of the training. Zoe's mention of a lack of engagement led me to inquire about what it was like to have people who did not want to engage in conversation. And if they did not engage, did they distract? Or did they help bring any views in opposition to what was being taught; expressing, hard feelings about the training course. Zoe replied:

From a personal standpoint, I thought it was really harmful that that individuals would like, take away from that experience for others and mostly like, it wasn't necessarily like outright opposition to the conversation, but it was like talking while these individuals were sharing their stories and being really insensitive to the fact that this was a vulnerable moment for that individual. And so, I at least in the time that I attended Citizenship@Mizzou, there 
wasn't anybody who outright came out and was yelling or creating that kind of atmosphere, but definitely attention was not always paid.

Zoe is referring to Whiteness as Property and the mistreatment of vulnerable voices. Those students who failed to actively listen to the counter-stories and engage with their peers or facilitators in C@M felt privileged enough to propagate a dismissive and superior disposition, further maintaining systems of marginalization. Notably, while Zoe did not recall vocally overt displays of opposition to those sharing their experiences, their lack of engagement and side conversations were subliminal displays of their negative disposition, opposition, and dissident superior attitudes. Behaviors of which, if unchecked through curricular reform, remain impediments to institutional reform and improved intergroup relations within the campus climate. Naturally, her responses prompts intrigue to know how Dr. Shonekan felt about the training course's designation as required and if, after facilitating it, she thought it should remain a required institutional response in practice.

Dr. Shonekan left Mizzou for a short period of time before returning during the summer of 2020 in a new associate dean capacity; of which, she was tasked with reviewing the progress and future of Citizenship@Mizzou. So, when asked if she thought it should remain a required institutional response in practice, she reported that she could not say much at the moment because she was not too sure about how the training course had changed after her departure. However, she made it very clear that she would be proposing something new to the course to take it to the next level and make it much more sustainable and meaningful. She remarked that what they did in 2016 was what could be done at the time but that it should be much more comprehensive and perhaps should be combined with Race and the American Story. On the other hand, Dr. Shonekan did share her perceptions about Race in the American Story being required or optional with more clarity.

\section{Race and the American Story}


Dr. Shonekan stated:

I think Race and the American story in my current thinking should remain optional. But I think there is a way to recreate a version of it, slim it down and distill it so that could be required for all students, all staff, and all faculty. Definitely students, I know it'll be a fight, but every department had all kinds of statements this this summer [2020], right? And I have collected all those statements. So, this is the time and, and I think there's potential in this moment, and I'll keep you posted on how that goes.

Contrary to the students who reported that Citizenship@Mizzou should be required, Professor Shonekan thought that Race and the American Story should remain optional but did not provide much rationale. However, she did allude to the possible creation of a slim-downed version of it that could be required for not only students, but all faculty and staff. Her responses about both curricular interventions indicated that this might become the new version of Citizenship@Mizzou. In doing so, she-like the student participants of this study above-recognized the likely resistance from students but also faculty and staff. To redress this dilemma, Shonekan referenced the many Diversity / social justice-based statements released by many of the departments during summer 2020, following the nationwide protests to the murders of George Floyd, Brianna Taylor, and Ahmad Aubrey at the hands of police and White vigilantes. These statements would be used to undergird the rational for requiring a new/revised intervention, as acting now would be most beneficial, given the current climate. It would allow faculty and staff to display their written statements with their actions to advance their own knowledge around institutional racism and racial unrest. But what are students who were enrolled in Race and The American Story saying about its designation to either be required or optional?

Luke stated: 
So personally, I think that it is vital for the class to be mandated class I think that every student in America should be taking a class like that. But I think particularly in a PWI, predominately White institutions that they need to be required. For me, there was no other class that I took in my undergrad experience that was more impactful, was more meaningful, was more eye opening. I thought it wouldn't even relatively work right for a White guy. And it just opened my eyes to my classmates that I grew up with, in a way that never would have happened if I hadn't had that experience and if I hadn't taken that class, so $100 \%$ I think I think it should be required.

As a White male, it is critical to understand his perceptions about a course that directly addresses race and racism throughout American history. He brought attention to the importance of PWIs requiring said content as he proclaimed it to be the most meaningful and impactful course he had taken, which forced him to investigate at the individual level-through his peers and readings - the nuanced lived experiences of Black people, as a part of the goal to opening minds, understanding the intersection of identities, and encouraging behaviors of social justice advocacy. In fact, Luke explicitly stated that if it were not for RAS, he would not have experienced such an impactful intergroup relations dialogue experience. Luke did express some of his concerns with requiring the course, stating:

The only thing that I worry about mandating it is that we will have students who treat it as an obligation and blow it off and don't approach it with an open mind as being required of them. And we have this really toxic culture, I think in schools across America, but including into this idea of the political correctness culture, right, this, idea that like these people are forcing us to be moved social justice warriors, you know, higher education and political correctness to them, right. And so that that is something that I would worry about is that it would be risky to do that. And people might discredit it because of that. But nonetheless, I think it should be mandatory because I think, regardless of how you approach it, that class 
forces you to be in a community with people who do not have the same experience that you do, and also forces you to critically think about evaluating your own privilege as, as a White person as a straight person, what, whatever. And so yeah, no, absolutely, I think it should be required.

Luke's perceptions about requiring Race and the American story are consistent with his peers' regarding C@M in the way that resistance is likely to occur. In as much as he believes it should be required by every student in America, he recognizes the disposition that people in positions of privilege and power are afforded to be dismissive and resistant to anything that seems to push against White normalcy and what has been determined as the standard for instruction; another instance where the notion of Whiteness of Property is noticed as alive and shaping the minds of those who have been taught to carry superior dispositions. Luke pointed to a social issue, he argued has created a toxic culture across academia, whereby some people from privilege backgrounds feel as though they are being forced to be politically correct and become advocates for social justice. Despite these claims, Luke's perspective was that the course would still force people to examine their gender identity, White identity and the privilege it yields, while coming to understand the lived experiences of Black people through counter-stories of peers and scholars studied throughout the course. If we consider the students' insistence on requiring both interventions, it would be important to know, from a faculty perspective, what is involved in requiring a course. Dr. Seagrave was asked to weigh in and share if this was a part of the plan and the assumed level of difficulty, it may take to do so at such a large university. Dr. Seagrave shared:

It can be a very difficult process to require a course university wide. And it's something that we are always thinking about doing. And it is difficult. So, I think our initial thought was, well there are pros and cons either way with the requirement because, you know, sometimes you get a lot of benefit from the voluntary aspect of it, you know, that students are maybe more open to participating and learning if they feel like they're not being forced to do it. But 
at the same time, I think a lot of the students who wouldn't voluntarily participate are the ones who need it the most, right? So, I think there's a need to have these courses be required? I think that's absolutely right. And I think administratively, especially in a big university or college, there would just be a lot of sensitivity to the implications of requiring a course. And, a danger is always that by the time it gets through the bureaucratic process, that it's been so kind of watered down or stripped down or something that it ends up not really having much of an effect. I think that's the danger. And so, the challenge is to get something robust and meaningful, all the way through the bureaucratic approval process. Or it's different at every university, how the approval works to require a course university wide. But for that, yeah, most campuses, you'd have to do all the faculty senates and university senate and all of that, the various levels of the faculty governance, and then also the administrative approvals in order to make that happen, so it's possible and something we're working towards doing.

Professor Seagrave, much like the students reported, was in favor of requiring Race and the American story despite his awareness that attempting to do so would cause some level of bedlam amongst the various levels in a bureaucratic approval process. He did confirm that the major concern of the curriculum developers would be the prospect of RAS becoming diluted and incapable of existing in its original concentrated and robust format; causing it to lose effectiveness. Analytically, his perspective regarding the presumed level of difficulty getting the course requirement approved in its original form aligns with Whiteness as Property and a Critique on Liberalism. The perceived existence of resistance and the dismantling of Race and the American Story to make it required is a direct result of a White controlled system that claims ownership to intellectual property and the nature of content people have the rights to formally learn. The palpability of a contemptuous bureaucratic system impeding the stringent race-focused course design of a diverse facultyled curricular reform effort, is a display of a disingenuous disposition that prioritizes Whiteness over the 
valuable inclusions of Black experiences. Dr. Seagrave's perspective, as a White man, magnifies the plausibility of an insincere bureaucracy, primarily concerned with the appeasement of dominant groups and donors to the university, for whom they do not wish to upset. The avoidance of upsetting said groups, with a curriculum that exposes the history of America's mistreatment of Black and Brown people, at the expense of transformational change for those from traditionally oppressed groups, is a Critique of Liberalism.

Ida Campbell-Jones, a Black woman who took Race in the American story with Dr. Shonekan, reported her unmitigated support for requiring RAS for all students. She declared:

Um, I definitely think that it's a class that should be mandatory for students, especially the way that it was taught at Mizzou. The class that I had was very diverse, both racially and gender wise. We had a very good mix of identities in that class. And the way that we discussed both racial disparities and histories that are not typically discussed within, I'm gonna say gen-ed courses, I think it's very necessary for students to take that class, because there are things that were deliberately skipped over in other classes that are in this course.

Of particular importance to this study is the nature of intergroup relations amongst those from diverse racial and social identity backgrounds with their White counterparts. Ida described the diverse nature of the identities in her class as being vital to the effectiveness of the pedagogical approach to use intergroup dialogue to drive conversation, mend or proactively mitigate the campus's racial unrest. In doing so, all students were afforded the opportunity to collaboratively explore and examine the racial disparities in America within a historical context. A context that otherwise, would be less likely to occur in - what Ida referred to as-gen-ed classes, where she perceived strict race/identity conversations were consciously avoided. When asked if she thought requiring RAS would take away from the learning experience, Ida stated:

That's almost half and half. I could see it taking away from the desire to learn if it were an entry level class. So, Mizzou has this thing called FIGS [Freshmen Interest Groups] that 
freshmen students have to take their first semester or first year that I've heard their participation is lackluster, but in classes that are needed to graduate, in general, if they can choose whenever they take it. I think there's more of a desire to learn because it's more of a leniency. Does that make sense?

Ida referenced Mizzou's existing Freshmen Interest Group (FIG) as a measure to perceive how students might respond if RAS were to be required in the same manner. She reported that participation in the FIGs was uninspiring; therefore, concluding that if students could choose when to take RAS, much like their required general education courses, students' desire to learn and be impacted by RAS is possible. As educators/practitioners are seeking to reform their institutions via the curriculum, it would be important to consider existing examples of how students perform or perceive their learning to be in courses currently being required. Contrary to the perceptions of most participants in this study, Dr. Stephen Graves reported why he is opposed to requiring RAS.

Dr. Graves declared strongly:

I think it should be optional. I don't believe in requiring students to take anything! I don't, If you're not interested, don't take it! If you have no interest in being open minded on racial issues, stay the hell out of the classroom! Then, when it comes to racial issues, those people do more damage than any good; they're just sitting back in the classroom making smart ass comments and holding on to their ridiculous ignorant perspectives! And they have no interest in changing their mind at all whatsoever! If you're not open, if you have no interest in changing your mind, stay the hell away from me and the hell away from my classroom for sure! Because I'm coming after you and it's gonna be my job to try to push you intellectually into changing your mind. But if you're that hard-headed, you are not open to changing your mind, stay the hell out of my classroom, that's for sure. So, and forcing students to take it, 
you're going to get too many and many more of those people in your class, if it's not required, than you're going to get those who, actually, genuinely want to take it.

Dr. Graves, essentially, called out the behaviors and attitudes of people who uphold aspects of Whiteness as Property, which ultimately undergird the palpability of the Permanence of Racism that is pervasive today. Particularly, the apathy towards becoming active learning participants to examine the historical racial issues of American history, is a form of resistance to reforming intergroup/race relations. Additionally, the failure to increase their understanding of non-dominant groups-in what should be a welcoming pluralistic society - through their experiential knowledge, is also an act of resistance, their complacency within an idle White supremacy, and their commitment to maintain a disposition of superiority, by being dismissive to the people and experiences of traditionally oppressed groups. Dr. Graves, was not adamantly opposed to forcing students who do not have an open-mind to having it changed in any way. We should also recognize that not all individuals, who initially display a lack of interest in such race-focused content are closed-minded and may be willing to engage from where they stand, while also paying attention to the voices, experiences, perspectives and presentations of the vulnerable. Dr. Graves was careful in his attempt to frame his thoughts, as to not dismiss the opportunity to teach those who may not be interested initially but are yet open-minded, as opposed to those who enter with no will to be influenced or embracing of diverse perspectives other than their own. Given the perceptibility that any given number of students might be resistant to institutional reform through education, a look at the findings on the perceptions about the institutional response / curricular reform efforts to change Mizzou's campus racial climate would be prudent to consider. 


\section{Perceptions about the institutional response / curricular reform efforts to change Mizzou's Campus Racial Climate}

This section will present the findings analyzed by participants involved with Citizenship@Mizzou followed by those who participated in Race and the American Story. Student participants primarily responded to the following prompts in their own way and not to any specific degree of exactness: 1) How do you perceive Race and the American Story to improve the institution's campus racial climate and how it could improve? 2) How well do you think the course influenced behaviors to combat and advocate or face race and racism outside the classroom? 3) What are your thoughts about the adequacy of this course as an institutional response to the campus racial climate issues following the protests of 2015? And similarly, faculty participants responded to the following responses: 1) What are your perceptions of what students who completed the course feel or are able to do as it pertains to race and the discontinuities of race dynamics on their campus and beyond? 2) What are your perceptions about how those who taught or helped to facilitate did in order to achieve student learning outcomes and what do you think was achieved? From their responses to these prompts and unique follow up questions, the foundation of their perceptions were captured through their experiences and stories shared. Jacob spoke about Citizenship@Mizzou.

\section{Citizenship@Mizzou}

Jacob:

I don't think it hurt anything. I don't think it made anything worse. I don't know. I wouldn't really consider Mizzou a bastion of cultural acceptance or something at this point still. So, we didn't change the world. But clearly the problems aren't over and they're not solved. It's hard for me to maybe answer this question in a kind of subjective way. I mean, of course, we didn't help that much, everything's fucking still going on [nationwide protests 
of summer 2020]. But I know that there are people that we touched and that we affected. So, there was some good done. And I guess anecdotally there's one girl that came up to us afterward and she was a White girl. She's, from the suburbs and I don't know really necessarily if this is a good thing or something, [but] she was wanting to be a part of the band and it just never really worked out. But she offered and said it kind of opened her eyes and she believed in this now. And I'm also a DJ at all the bars in town that're predominantly Black. I see her all the time with a bunch of Black friends and stuff. So, I don't know what's going on in her head necessarily, but that seems like kind of a cool sign. She maybe opened herself up a little bit. So, yeah, small things like that. I guess we can say that there was some change, but we didn't say the world.

As Jacob reflected on the impact C@M had on the campus racial climate, he used race to describe a White student who, following the training course, voluntarily reported her pleasure with her experience and the work being done to create positive attitudes towards embracing Mizzou's core citizenship values. He reports that she wants to be involved with the band; analytically, this was viewed as her way of wanting to align her new-found beliefs from C@M with her actions, as a way to give back or be a part of the mission to cultivate a more equitable campus racial climate. Jacob also witnessed the out of class intergroup relations the girl had with several Black student peers in a predominately Black social space. Personal relationships do not undoubtedly indicate one's collective embrace, understanding and appreciation for Black, indigenous, or people of color, nor their desire to advocate for said identities. However, it does indicate a willingness to engage and maintain positive intergroup relations through the building of multiple social networks within the human ecological system. While Jacob believed in the mission of C@M and believed it helped some people, he did not perceive it to have made a massive impact on the campus racial climate, but rather a good one. He described how he perceived the institution's response with C@M to have been by saying the following: 
Sure. I think that obviously, a lot more should have been done from a leadership position, from the chancellor and the institution of Mizzou. I kind of believe that these institutions need to lead by example. And Mizzou didn't really lead by example, it felt like they just found the head of the Black Studies Department and had her find a multiracial group of students and talk to people as a band aid. So, like I said we tried to do as we could, but ultimately, the university weren't making a habit of supporting their Black students in meaningful ways. So, obviously citizenship [C@M] wasn't a bad thing. It wasn't a hurtful thing, but it wasn't the answer that we all needed or wanted so yeah. Everybody's got their opinion about what Mizzou should have done. I would have done things differently if I was Chancellor.

Jacob's frustrations align with a Critique of Liberalism and Interest Convergence regarding the institution's response with C@M as a form of institutional reform. Specifically, he recounted on the perceived limited and lack of ongoing and noticeable support for Black students under duress, he thought Mizzou officials; namely, the chancellor, should have be given. To this end, Jacob perceived his university's administration to have engaged in a bit of Interest Convergence by requesting a faculty member's help to implement a curricular intervention that might mitigate the discontinuities in race relations at the time. However, the interventions would, both, come at a slow pace and be administered in less than direct manner in order not to create an uproar amongst the campus's dominant population of students; of which, is consistent with a Critique on Liberalism. To be clear, Jacob did not place any blame or lack of effort on the scholarly work of Dr. Shonekan and her peers in designing Citizenship@Mizzou, but rather he perceived the senior leadership as passing the buck (responsibility) to one faculty member to remedy a campus-wide racial unrest issue, sparked by the national outrage of police brutality and murders committed on Black citizens. Analytically, Jacob clearly indicated that senior leadership should have done a better job with their treatment of the individuals with vulnerable voices, experiences, perspectives, and presentations at 
Mizzou. So how did participants perceive C@M to have impacted or influenced its attendees towards changed behaviors and a desire to advocate for social justice? Zoe, described her perceptions of her peers who had taken C@M, outside of the training, which were rather disheartening. Zoe shared:

I would say minimally, it had a very minimal impact because obviously I'm very white passing. So, people don't realize that I'm in the room when they choose to make racist comments, but [minimally] based off of the frequency of racist comments that I heard throughout my freshman year after we had all been required to take that course. And there were people who lived in the dorms with me who would just make snide comments about this, that or the other. Based off the frequency that racism was still very present after having taken the course, I can't say that it was all that effective.

Zoe's perception of C@M on her peers' ability to change following the course is consistent supports the assertion that there is a Permanence of Racism in American institutions. In this case, it moved through Mizzou's human ecological microsystems, as students socially interacted outside of class and in their residence halls amongst peers, of which they believed to be a part of the same dominant racial group. On the contrary, because Zoe's physical appearance makes her look as if she is White, she bore witness to the unabated and cruel racist rhetoric spewed about Black people within her residence hall microsystem. When Zoe was asked what she did in this particular microsystem of hers, to combat racist rhetoric; she said the following:

I can't seem to keep myself quiet in these situations. Usually, I really questioned why it is they think like that, cuz usually it would be stereotyping or racist slurs and I typically asked questions like "why do you think that that's okay to say, what formed your perceptions to make it that this is true of all of that racial group," I just really challenged them to think 
about why it is that they feel so free and just expressing that thought when it's not an acceptable thought; just encouraging deeper thought about the actions and comments.

Zoe's experience within the microsystem, where she lived on campus, is another display of how a Permanence of Racism is interwoven throughout our social interactions, systems, and structures. It was interesting to understand how Zoe managed to face and combat overt racism and use it as an opportunity to provoke her White peers' meaningful reflection and Critical consciousness, regarding their discriminatory ideology and rhetoric that propagates a superior disposition over Black, indigenous, and people of color. Ideally, what Zoe did-encouraging deeper thought about their actions and comments-is a part of what advocating for social justice looks like; it is what has the power to move people towards the attainment of a more equity-minded approach to their daily social interactions. Zoe did not report in her recount of said incidents, that C@M training led her to challenge the power of White Privilege in the manner she did, but an analysis of the interview in totality, would suggest that she is inspired to act and create opportunities to advance knowledge when she is enlightened. Therefore, it can be inferred that C@M--even for what it lacked in her opinion-prompted Zoe to stay engaged with sharing the counterstories of the vulnerable. In fact, as a tour guide on campus, she has developed a special history training program for tour guides she hopes will extend to guests and families. Mizzou has five social justice centers on campus, each with their own rich history of why they were creative by influential individuals on Mizzou's campus. To this end, Zoe said the goal of the training is to prepare every tour guide to tell the stories of these individuals, make sure their stories are heard and are granted the stage they deserve to pay homage to them for paving the way for improved relations at Mizzou throughout the years of Mizzou's existence and tumultuous history with racism. A part of the goal was to equip tour guides with the tools to understand and share Mizzou's story with race relations within a historical context. Analytically, Zoe was adamantly concerned with the treatment of the vulnerable and how their experiences, perspectives, and presentations influenced Mizzou's structures and vice versa. By designing the formalized tour guide 
training, it was concluded that it was yet another measure of institutional reform, but at the department level. Otherwise, when Zoe described her perceptions about the adequacy of C@M as an institutional response to the campus racial climate issues at Mizzou, she stated:

I don't know, so, I'm a tour guide at the university and I've been thoroughly educated about the events that transpired in 2015. After having been educated about the events in 2015, and then having experiences in Citizenship@Mizzou, and a year and a half later after everything happened, it really truly felt like the administration was just looking at it like a band-aid to be like “don't worry we're talking about it, we're having a conversation about it, we just don't know how" type thing, and I think that showed in their execution. So essentially, I think that if people were willing to engage in that conversation, or if it had been directed more directly, it would have been more successful, but I don't think that as much thought went into the method of like delivering that message.

Analytically speaking, Zoe pointed to a Critique in Liberalism on the part of Mizzou's administration, who she felt did not have an adequate response with Citizenship@Mizzou. She perceived the institution's response as a mere band-aid to the Black students' outcry for mitigation to the racial hostility ensued over time, that often ended with the administration's nihilism or apathy to implement transformational changes. The sluggish and slow-paced liberal efforts were-in Zoe's view-somewhat ineffective in redressing Mizzou's campus racial climate issues, given the indirectness of the intervention to tackle the permanence of racism at Mizzou. Essentially, she argued that C@M would have been more successful if people were more willing to engage more stringently with race/identity dynamics through intergroup dialogue. On a scale of 1-10, 10 being very much landed on target, Zoe gave C@M a 2.53 in terms of its effectiveness to being an institutional response to the protests of 2015. Luke, who took both C@M and Race and the American Story, weighed in with his perceptions about the adequacy of C@M as an institutional response to Mizzou's campus racial climate issues, stating: 
So, the diversity and inclusivity training, very first steps very surface level. It was led by Dr. Shonekan and she's fantastic and that kind of all on her own. I mean, that is something that, I think is the first step that the school took. That makes them look better but doesn't do a whole lot to really change the racial environment on campus. I think there are more systemic reforms to higher education that we need in order to make some more racially equitable spaces. And that's not just at the University of Missouri that's across the country, and also with the ways that we police students of color and particularly Black students. I think that that is something that needs to be dramatically changed. If not, I don't think we should have cops on campus, especially cops that are employed by the school. I think it just serves to over criminalize Black students. And so, I think the school could be doing a lot more meaningful things to address racial inequity on campus that they refuse to do. And, you know, policing is just one aspect of it.

Luke quickly credited Dr. Shonekan and her fantastic work but shed light on Mizzou's administration for engaging in Interest Convergence, because of the inadequacy of C@M as an effective institutional response for changing the racial environment. He felt that it was a first step that made the school/administrators look better but that the administration failed to consider or bring about additional systemic reforms to produce racially equitable spaces. Specifically, he referenced a need to reform how students are policed; particularly, Black students who have bare the brunt of an over-criminalized approach to policing them. It appeared that Luke was not satisfied with C@M as the institution's initial response and hoped for a more holistic approach to institutional reform.

When Dr. Shonekan was asked to share her perceptions about how well she thought those who facilitated C@M did to achieve student learning outcomes, she expressed some level of difficulty with being able to provide this in an y measurable way. For example, she expressed that given the limited twohour time span, some students slouched in the back of the auditorium and were not interested. Likewise, 
Professor Shonekan recognized that there will always be racists and homophobic students on campus because of their familial upbringing that has engrained it as a part of their culture; thus, she said it is difficult to break through that learned culture in only two hours. However, she believed that there were more students who opened their eyes to the seriousness of embracing the core values and contending against the campus's cases of racism, sexism or homophobia. The measure she used to conclude students' acceptance of C@M and willingness to engage with the material was when students offered themselves to be a part of the band or requested to discuss and analyze certain songs; it was a sign of their enthusiasm. Analytically, Dr. Shonekan was not naïve in her attempt to launch Mizzou's initial curricular reform in response to the 2015 protests, by thinking that all students would abandon their long-standing belief systems and dispositions and be transformed in two hours. Nevertheless, she remained steadfast in her attempts to open the minds of those who were willing to engage; knowing that she would have to develop another curricular intervention to delve deeper with the subject matter and spend more time throughout the semester in a formalized course for academic credit. The course became known as Race and the American Story.

\section{Race and the American Story at Mizzou and Beyond}

First, let us make note of the outgrowth of Race and the American Story because of its productivity and embrace from students and faculty across the country, before returning to the individual accounts of research participants. After a successful five section debut at the University of Missouri-Mizzou in spring 2018, RAS expanded its reach to students at the University of Massachusetts, Amherst and Arizona State University in the spring of 2019; where Dr. Shonekan and Dr. Seagrave began working respectively. In the spring of 2019, the professors decided to bring all students enrolled in RAS at all three institutions together for an Inaugural Race and the American Story Symposium in April 2019. Their aspiration for the RAS project, is that its symposium travel to a different participating school. Unfortunately, after a successful inaugural year, the Coronavirus (COVID-19) thwarted efforts to hold it at their newest participating 
institution, University of Memphis; instead, the symposium was held virtually during the summer of 2020. According to Dr. Seagrave, in 2021, the RAS project was slated to welcome five new institutions; they are: University of Memphis, Rhodes College, University of Wisconsin-Eau Claire, Linfield College in Oregon, and Sorbonne University in Paris. The addition would bring a total of eight colleges and universities together, that all teach Race and the American Story in the exact same manner; with the exception of Sorbonne University, which will tailor theirs to French colonial history. The main variations are the number of credit hours it is listed for and the faculty teaching it. Throughout the course, students from all participating campuses occasionally interact, virtually, via zoom and on the website's discussion boards to share ideas, experiences, and perspectives about various discussion prompts posted.

Therefore, when Dr. Shonekan was asked what she perceived was done well and what was achieved by Race and the American Story, she provided us with the broad scope of how the course has been expanded to universities across the United States, as one measure of achievement that was executed well. Additionally, she shared how their students used what was learned in RAS, to become involved in a special summer engagement fellowship program to complete assignments to further aid and display students' learning outcomes. Recent enrollees of RAS who apply and are accepted into the fellowship are paid a stipend to select a topic they were activated by in the class or something they feel passionate about, to create a project that includes an audience. The project, for example, could be delivered like a television show, a group podcast series, film etc. to engage with and advance knowledge around what was presented in RAS and beyond. More specifically, Dr. Shonekan described existing projects of summer 2020 this way:

Some of them are doing race, woman, and politics. So, they're taking Shirley Chisholm they're talking about Stacey Abrams, they're talking about Angela Davis; that's a series and I think the summer engagement program is a really nice way of showing what can happen to students after Race and the American story. They've spent the whole spring semester reading all this stuff, talking about all this stuff and now they want to spend three hours on 
the film Get Out to just talk about what is the sunken place, what does that mean, and how is that reflected in contemporary culture? We've got one student who's looking at sketches, satire and political sketches, he's looking at, In Living Color, Key and Peele and Chappelle's Show and talking about how humor and satire have deep connotations in contemporary political life. So again, I bring this up Bruce, because I think the summer engagement program is a goodwill fellowship program, it is a good way of seeing what can come after Race and the American story. Each of the 10 students, come from the three campuses. I think we have four from UMass, three from Mizzou, three from ASU, and they're all coming together to bring their audiences to each other. Some of them have had 25 people in the room talking about Black queer identity, and Adam and I just sit there and we just watch what goes on and they're referring to the readings that they encountered in the class. They're referring to some of the music we talked about. So, I think that we can get an evaluation on the course that says the course is great, but when we see them continue the conversation in their own zoom rooms. That is that is a sign of the impact of the course.

Inherent with students' unique displays of their learning outcomes, is their ability to change or become facilitators of social justice/institutional reform. Not only do we come to understand what students have learned or are able to do in the realm of social justice reform, we also are afforded an opportunity to witness students' ability to change, advance their knowledge on race/identity dynamics, or become change agents and advocates within the spaces they enter. Many of the student perceptions about themselves or their peers reported in the previous section and later in this one, center around the notion of these curricular interventions and their effectiveness on the students for which they were designed. This approach helps us to understand if and how students are being transformed, displaying equityminded behaviors and approaches to structural systems and engaging in efforts to cultivate and maintain a 
more inclusive campus racial climate. Further analysis highlights the connectedness of Dr. Shonekan's mention of historical figures, visual texts, and individuals with intersecting identities to her students' desire to investigate at the individual level, their nuanced lived experiences. The students at Mizzou were able to engage with a diverse group of students from multiple racial and social identities and backgrounds across the nation to Critically examine and discuss, for example, the ways ones' Black and queer identities intersect and are treated within a society that clings to the legacies of racism, sexism, homophobia, etc. Moreover, to also pay attention to the counter-stories and journeys of Black women activists and politicians over time, to analyze visual and audio texts and discuss their symbolism, ideological perspectives espoused, and their influence on American systems and individuals. Dr. Shonekan argued that while course evaluation data is good, seeing the students' transformative work towards advancing knowledge first-hand, was the true sign of the RAS' meaningful and transformational impact on students and institutional reform. In narrowing back to the individual perceptions of this study's participants, Luke shared his perspectives on RAS to improve Mizzou's campus racial climate and the adequacy of it as an institutional response to student protests. Luke stated:

I think it is a really good start. I think it is. I think it was a really, really good course. I wish it were required for all Mizzou students because I can guarantee you that class has had more value on and impact on the way that students think about their relationship with other students; specifically, White students and students of color more than any other course at the university. I can't overstate how impactful it was personally. And I, I do think that a lot of the things that we covered in that class are things that students of color and particularly Black students already know and have already experienced. So, like I said, I do think the course is particularly important for White students to take a class like that. Institutionally, I'm so glad that they made that course available. 
Luke's view that RAS should be required and his proclamation that it was the only course at Mizzou to prompt students to analyze their intergroup relations with peers, reaffirms the vital role of the intergroup relations dialogue approach to combating campus racial climate issues through educational reforms. Again, Luke, as a White man, expressed how the course was particularly impactful for him and his White peers. He also paid attention to how Black people and students of color, in the course, were positioned in terms of the intergroup learning process; namely, because much of the content was about things they already knew or experienced. However, he found it important to have access to their contributions to elicit productive conversations, so that existing mindsets and dispositions-held by his White peers-of White normalcy as superior to others can be diminished. Luke's next response below, does not directly address the questions posed; however, it is a first-hand display of that which Race and the American Story prepares its students to do. In it, Luke essentially Critically examined race relations at Mizzou and in America, by describing their current status and progress on combating racism and systemically addressing the demands of Concerned Student 1950 and the unjust murders of Black individuals at the hands of White police and vigilantes. It is included in the analysis because it is the essence of how Race and the American Story interrogates race and racism in America and Mizzou, while Critically analyzing them within historical context. Explicitly and analytically, Luke argued:

I think they [University administration] need to do a lot more if they want to address the kinds of systemic inequities that dramatically Concern Student 1950 brought to them. I think we're still experiencing this systemic racism. And particularly in higher-ed, I mean, right now as a result of George Floyd protests, there's a nationwide movement to remove these statues and icons that represent White supremacy and racism and there's a movement on campus to remove one. There's a statue of Thomas Jefferson on our campus and it is disempowering for students of color and for Black students in particular to see and I can't imagine walking past a person who enslave and raped people. And specifically, 
for Black people, I can't imagine how frustrating and harmful and just aggravating that is in a learning environment that claims to be an institution that prides itself in diversity and inclusivity. And we've had a response from our current president and student activists after they sat down and voiced their concerns, that was pathetic. He basically sent a twosentence email that said that person's contributions to our country in the world were seminal and deserve to be celebrated. And to me that is just profoundly ignorant that yes, okay, Jefferson helped write our founding documents, but those founding documents, uprooted Black people and doesn't deserve to be celebrated. He did something and he, you know, he helped to you found a version of democracy that was for land owning Whites. And at the end of the day, he was a racist, and he was a rapist. And we shouldn't celebrate those kinds of people. And we continue to do so. And it continues to perpetuate not only racism, but interpersonal racism.

Analytically speaking, Luke became attentive to how power operates and is challenged within higher education, that has been influenced by the inequities in criminal justice across America. For example, he references the resurgence of the public outcry and demand to remove historical structures rooted within notions of racism, White supremacy, and oppression; one of which is Thomas Jefferson at Mizzou. Luke described the negative impact such structures continue to have on the social interactions and morale of the Human ecological system and the Black people with in it. His frustrations are reflective of his concern for the treatment of Luke's Black and students of color counterparts and how those structures are symbols of disempowerment for them; having to be constantly reminded of how White slave owners and rapists are celebrated and held in high regard, because of their role in the foundation of America. Nonetheless, he pointed out the huge disruption the Thomas Jefferson structure presumably has on a students' ability to perform well academically; impacting yet another area of a student's microsystem that engenders feelings of justified antagonism towards those who fail to recognize the dreadful role Jefferson had on the lives of 
Black people. It is concluded in the analysis that Luke did not perceive his university president's treatment of the vulnerable voices, who felt angered and oppressed by the statute as favorable. In fact, he viewed the president's response to the students and limited in scope and dismissive; ultimately justifying the preserving of a racist and rapist historical figure. In such case, Luke felt his president prioritized the historical legacy of Thomas Jefferson's role in writing America's foundational documents above the counter-stories and experiences of Black people historically and presently at Mizzou. Such structural allowances serve to perpetuate a Permanence of Racism within the campus climate and can lead to feelings of marginalization and unhealthy intergroup relations within the human ecological system. Luke sums up his perceptions of Mizzou's overall institutional response to student demands along with those about Race and the American Story, stating:

It's honestly just really frustrating that the administration has learned so little you know. Concerned Student 1950 was so important, and I think it was brought about by what a lot of folks in Missouri called the children of Ferguson right? There were people that were radicalized by the movement in Ferguson and by Michael Brown's murder, and I don't want to downplay what they did, and how important that was to our campus culture, because it brought us forward. It did, it changed things on this campus, this class is a result, and there was a lot that happened as a result, but not enough. And I think you can see it today, we're still pushing for more, and we're still getting a milk toast basic response at best.

Luke expressed strong emotions of frustration with Mizzou's administration for-what he called-a milk toast response to the painstaking activism Concerned Students 1950 did in demanding institutional reform. He shed light on the magnitude of the movement that was radicalized by Michael Brown's murder in Ferguson as a way to draw a connection to how external circumstances influence an institution's macrosystems and how Techo-systems (e.g. media, social media, phones, etc.) can lead to campus reform. 
Namely, he credits the protests and demands of CS1950 and Race and the American Story for bringing Mizzou forward and creating transformational change to the campus racial climate but thought there should be more. In like fashion, Ida Campbell-Jones, gave her perspectives on RAS improving the institution's campus racial climate and how she might see it improved. Ida stated:

So now, I feel like the class definitely benefited the students. I think a lot of the students in the class were juniors and seniors at the time. So, I think that it definitely benefited them in the way that they view Race Relations at Mizzou. And going into the job market after graduation, I think that was really good, especially personally for me. To me, it was a really good course in my undergraduate career and it gave me a better jump of where to and what to look at post grad, going into higher education. But I can only speak from my experience with the University of Missouri; but a lot of both faculty and administrators at Mizzou have a hard time accepting that there are systemic racial issues at Mizzou. And I think that the class, by naming those issues, would be a great start at improving race relations. Because a lot of people do have a hard time even identifying where there is racism. So, I do think, especially if the class were to be mandatory, that it would be a great start to identifying where the problems are and have been at Mizzou indefinitely, if it were at other institutions that would do the same.

One aspect of identity that has not surfaced much in the data is age. Much of what has been reported has only focused on C@M as being required for freshmen and transfer students. While class standing cannot automatically determine ones' age for us, given non-traditional students who may be included, we can assume that the majority of those who are juniors and seniors are older than the freshmen and closer to determining their future major discipline or career goals. And their involvement with RAS might influence those decisions. Ida, was the only participant to mention that her section of RAS was dominated by juniors and seniors, of whom she perceived the course to be a significant benefit given their close proximity to 
entering the workforce or graduate school. For example, Ida remarked about how RAS aided her in knowing what to look for, from a Critical perspective, in determining her graduate program in higher education. Analytically, it would seem that ones' age or class standing, paired with their exposure to a course like RAS, could force an individual to be more inclined to explore their own identity development, as they begin to investigate the historical legacies of power and oppression and how their culture was positioned in it. Consequently, the transformational power associated with one taking a course like RAS, is likely to influence people's identity and cause them to self-reflect about their desired journey. For some, it might mean becoming more equity-minded in their decision-making and how they will decide to engage this work, based on what they discover about themselves and their cultural past. Or, for others, it may mean reinforcing the pre-conceived notions about their culture and that of others, which do not entice them to position themselves as future change agents or producers of knowledge around the ideals learned in RAS. Additionally, Ida's perception that a portion of Mizzou's faculty and administrators grappled with accepting the reality of systemic racism amid the campus, can be associated with the Permanence of Racism and a failure to listen to the voices of those who are saying they are oppressed and feel marginalized. It reinforces the notion that Black people, indigenous peoples, people of color and LGBTQ+ people are the voices often less heard; thus, not only do these groups have to endure the pain, exclusion, and marginalization they experience, but they must fight to make White people believe it, provide displays so they can see it, take the time to teach it, and then fight to change it interpersonally and structurally. Hence, Ida reconfirmed her belief that RAS should be mandatory and used to help faculty and administrators work with students to discover and name the systemic inequities and racism permeating the campus. Furthermore, Ida's perception about the institution's response to Mizzou's 2015 protest were: So in terms of time, I think it took too long for the class to come to fruition. But I know that's not at the fault of anybody but administration. I wish that it would have been offered to more students. And I wish that it would have been a three-credit course instead 
of a one credit course. But I do really appreciate it for what it was. Because the students that took the class and the professors that taught the class, were all interested and passionate about learning more about race and racism in America and in Missouri. So, I think, as the first kind of class for race and the American story, I think that it was great for what it was. But we definitely need more. It's something about having to choose, or having the students have the ability to choose if they want to learn about race and racism. That's just not, it's not acceptable to me. I think everybody needs to take this course. But it just took too long, I think it was my sophomore year 2015 and I didn't take this class until...well, the class wasn't available until 2018. The University of Missouri is not doing enough to me to make sure that their students are educated on race and racism. It shouldn't just be up to faculty. I do think that this class did add a lot of pressure, unnecessary pressure, on the faculty that taught it just because there was not much support for these types of classes outside of the departments in which it was offered.

Time was immediately regarded as an impediment in the students fight for social justice and an improved campus racial climate. Ida perceived the Mizzou administration's response as much too late, not enough to ensure all students are educated on race and racism and yielding an unnecessary burden on a limited number of passionate faculty. Ida and Mizzou students were not able to take RAS until three-years following the initial campus unrest and Black student demands for curricular reform. Although RAS was not the initial institutional response, it was the outgrowth of C@M, the initial response, of which, it was not strictly focused on the events of 2015 , nor race and racism explicitly. But to Ida, who took RAS, it is apparent that she perceived it as what Mizzou needed by and large to combat the discontinuities in race relations amid the campus climate. Moreover, Ida's Critique on Liberalism and the slow-paced curricular reform - that should not have been on the backs of a handful of faculty to create-also exposed her frustrations that not requiring RAS would even be a discussion. In fact, she clearly took issue with the 
administration's flawed perspective that students should have an option to learn about race and racism. Again, Ida's perspectives about her administrations' willingness to make learning about race and racism optional and lack of a timely and more race-focused curricular reform, is consistent with the assumptions of Whiteness as Property. In other words, those in possession of the power to withhold race-focused content, that may counter the White standardized stories traditionally taught, are essentially reinforcing dispositions of superiority and who has the right to learn such content or be dismissive of it, along with the experiences of the people it continues to oppress. Naturally, such control over the intellectual domain, underpins the notion of a society that will forever maintain a Permanence of Racism without significant challenge to elicit transformational and systemic change.

On the other hand, in terms of the work that the faculty, who created Race and the American Story did, Dr. Seagrave shared his perceptions about how those who taught RAS did to achieve student learning outcomes and what he thought was achieved. Seagrave stated:

I think that the learning outcomes of better understanding the history of conversations about race in America achieving that better understanding; I think that's something that across the board through all of our sections and back into teaching that, that we've definitely achieved more historical understanding and awareness of how our situation today has grown out of our past in American history. I think that's something we've definitely achieved. I think that's one kind of learning outcome point to the other one, or another one being to achieve broader awareness of other student perspectives, or other faculty perspectives, on issues of race. I think that's something too, that all of our faculty have been able to achieve and a lot of that is through the discussion, pedagogy, and through our symposiums and meetings. When we meet in person together, we achieve a general broadening of minds, to see other people's perspectives. So that is what I think we've been really successful at doing. There's always room for improvement, though. I 
think it's something that you have to continue working at, you're not going to knock it out of the park overnight with any of these courses and these initiatives, you've got to work at it for a period of time and be open to improving and not think that you've got the final solution right away.

Professor Seagrave attributed a portion of what was achieved in RAS to understanding the story of race in America within a historical context, which increased student and faculty awareness of how their current situations are an outgrowth of that past. Analytically, this is noted as their attentiveness to the social construction of categories, within a historical context, to determine how systems have been influenced by said constructions and vice versa. It was their attention to power dynamics and how they have been challenged. Likewise, it was the faculty's adoption of an intergroup relations dialogue approach to facilitate in depth discussions that created an academic microsystem; whereby, students and faculty could expand their awareness of others' perspectives. Analysis suggests an interactive approach as contributing to the kinds of social interactions occurring within the human ecological system, that possess the ability to improve the tense interactions that were plaguing the racial climate. In summation, Dr. Seagrave described very positive perceptions about RAS' faculty and student participants on the overall purpose of creating transformational change within Mizzou's campus climate. He recognized that their efforts could always use improvement and recommends that people remain open-minded and do not expect to create an antidote overnight, but that it takes time to know what modifications need to be made. From an analytical perspective, Dr. Seagrave was, both, being and advocating for being reflexive on the journey to creating curricular reforms. In other words, it is important for practitioners/educators to constantly be Critically self-aware and reflective of their approaches to persistently produce better versions of their pedagogical strategies over time. Likewise, Seagrave reflected more deeply about how he perceived his students' utilizing what they learned from RAS, as it pertains to the discontinuities of race dynamics at Mizzou and beyond, stating: 
I think they feel better equipped and empowered to bring about change on campus and in their communities. As a result of the course, I've definitely noticed that and had gotten that feedback from a number of students. And most students that I've talked to, feel like it's very eye opening. And particularly in my experience, for many of the White students in my classes, they've thought that it opened up a whole world for them that they didn't know about before. So, that has been really a good outcome of the course for students. Overwhelmingly the response of students has been extremely positive and just energizing; I think they come out of the course feeling really energized. They've met people and befriended people who, who are different from them and who they might not have been friends with otherwise. They feel like they're part of a common cause and it's really important. And, they feel like they have the knowledge for making change. So, that's been so far the feedback, overwhelming feedback I've gotten; students really feel empowered and feel like they have a world opened up to them that they can try to change.

Seagrave's response, indicated that his students displayed their ability to feel empowered and equipped to advocate for social change. And for his White students, he reported that they were able to function with an open mind about their peers, who look and have experiences very different from their own, as evidenced by their friendly and ongoing intergroup relations following the course. The White students who reported that they felt as though they became opened up to a whole new world, are becoming identity developed, given their discoveries and new-found desires to advocate for social change and institutional reform. Additionally, White students seemed to be positively influenced around notions of transformative change after being exposed to non-traditional curricula using a Critical race lens to explore and by paying attention to the counter-stories of their Black, indigenous, or students of color peers. While Dr. Seagrave reported positive learning and behavioral outcomes for his White students after taking RAS as an optional course, Dr. Stephen Graves provided his perceptions about the possible cognitive and behavioral outcomes 
of another portion of White students, who might not be as prone towards improved intergroup relations, combating racism and condemning behaviors and practices that uphold systemic inequities. In like manner, Dr. Stephen Graves described some positive student outcomes from his point of view as a faculty member, stating:

There are definitely some students who left that classroom with a whole different perspective and ideas. Ideas that they had never heard before and heard their students and other fellow students say things that they didn't agree with and didn't understand how they could have that kind of opinion. They are now going to leave that classroom with a little bit better understanding about why those students think that certain way. And maybe we can get to them and change their opinions or perhaps not. There are definitely some students who walked out of there, who are even more passionate about racism and how it is in America. And they've changed their research and have changed their professional goals because of it, for sure, because they've been introduced to different ideas that they didn't think of, and now they want to reincorporate those into their major into professional careers.

A shift towards social change thinking and advocacy behaviors were reported by Dr. Graves, as he perceived his students to engender signs of developing a deeper understanding about the varying traditional and counter-story perspectives held by their peers and examined in the course content. Some students were led to either pinpoint or embrace the new-found perspectives, experiences and opinions of the intergroup mix of identities represented in the class. Consequently, the course content and intergroup dialogues, afforded by RAS, prompted some participants to change their research or professional career path after being reflexive about their values and ideologies. We can infer, some individuals became more aligned towards equity-minded attitudes and behaviors and needed to re-center their life's mission around the new discoveries uncovered in RAS. It also seems that the experience Graves described is an example of 
the utility of creating spaces where identity exploration leads to individual identity development. Although, this information is helpful to the overarching transformative mission of RAS as an effective institutional curricular reform, that is not the case for all students. To maintain the richness of Dr. Graves' thick descriptions, it was necessary to include the full scope of his personal analysis around teaching students who are resistant to actively and openly listening to the voices, perspectives, experiences, and scholarship of people from traditionally marginalized groups. In a very forthright manner, Dr. Graves proclaimed:

But there is still and will always have been and will always be a certain perspective, a certain percentage of White and sometimes non-black students who still don't want to hear that crap, who still think that the country is on the right path and doing just fine and that racial matters are overblown, and a lot of the ideas and stuff that they hear early in the semester, just do nothing but confirm that reading Thomas Jefferson say that "there's inherent natural differences between Blacks and Whites, and that Blacks smell funny and that [inaudible] their hair and they're over sexualized," for some of my students reading that, it just does nothing but confirm what they have already been taught and have learned their entire lives! And the ignorant crap, they've heard their parents and their pastors and their friends say over and over again! And at that point for some of them, that's all they wanted to know! And that's all that they were! They heard nothing else all semester! They heard Thomas Jefferson said that Blacks and Whites are inherently separate and Blacks are inherently inferior and smell different and over sexualized and everything else that is, and they're going to walk away highly focused on nothing but that alone! Because it reinforces their already ignorant and delusional beliefs! And then for some people, their ignorant, delusional beliefs, that's all they have. 
Critically speaking, Dr. Graves described the assumptions of Whiteness as Property and a Permanence of Racism to explain how not all White students will be accepting of or cognitively and behaviorally transformed by their experience with Race and the American Story. On the contrary, Graves contends that some White students, upon hearing or learning about historical forms of racism and oppression, might be reinforced by the longstanding generationally maintained dispositions that Whiteness is the standard of normalcy. Thus, further grounding, reaffirming and committing to the disposition that Black people and their ideologies are inherently inferior to that of White people. These individuals possess a lack of concern for the counter-stories of their non-White peers and can serve as the biggest impediment to improved intergroup relations that could bring-forth a calm racial climate at Mizzou; undergirding their right to a superior and dismissive disposition that makes racism a mainstay in the structuring of American systems and institutions. Dr. Graves further described his perspective on White resistance to RAS for some by expounding on how he perceived their cognitive dissonance being rationalized; he argued strongly that:

They're going to cling to those ridiculous beliefs more than anything else and trying to then tear down those walls by introducing Black things and other topics, just not even brushed the surface of the already deep held ignorant beliefs that they already have. And for some of them even hearing the opinions of the Black students don't change a goddamn thing for some, it doesn't. It just more further encapsulates the idea that they already have. "Well, they're Black, what would you like them to say, Well, of course, they feel that way. And of course, they don't think it's fair? And what do they expect? What do they expect to happen? They've been enslaved the entire time, and they're naturally inferior. So of course, they're not satisfied." And sure, not once, are they trying to actually tackle and challenge the opinions and ideas? And anytime you talked about why is everything inherently unfair and unequal in this day and age. They'll just be like "Well, the founding fathers said that they're inherently unequal and unfair, and everything else isn't. Thomas 
Jefferson was a really smart guy and hell if he couldn't figure it out, what do you want me to do is I'm just a, I'm just a guy. You know, Thomas Jefferson was this great philosopher and built this country and he knew better and he knew what America could do and if he couldn't figure out the racial problem, then what the hell you I'm going to do about the racial problem. So, there's definitely still always has been, always will be a large portion of the non-Black experience, who will just fully delve deeper into those opinions and ideas. And those are the students and people that we can't do anything about. The best thing we can do about those students is just try to reach the ones that we can and hope that they can hold those other students accountable by whatever means necessary. Hopefully, maybe through public humiliation, I think is always the best remedy for that.

Much like the previous response from Dr. Graves, the above response further describes the assumptions of Whiteness and Property and a Permanence of Racism for the same reasons provided earlier. Additionally, Graves explains the cognitive rationalization of an individual who ascribes to said White supremist ideologies as them wanting to relieve themselves from any advocacy duties associated with dismantling oppressive systems and social networks. This rejection and disinterest held by some, is done on the basis that such a "highly celebrated American figure," Thomas Jefferson, philosophized that Black people were innately born as inferior to White people; thus, there would be no need to attempt to undue what is naturally a fact. However, Dr. Graves was/is not in opposition to teaching White students of course, but rather he called out existing forms of defiance that are inevitably maintained by those who do not wish to dismantle idle White supremacy/racism. 


\section{Conclusion of findings}

Throughout this chapter, various documents germane to the case study, along with the perceptions of student and faculty participants of the study were analyzed. The analysis of participant responses was conducted within four unique themes emergent from the completion of the data coding process. The four themes chosen to organize the data sources' responses are: 1) Process of Curricular Reform Implementation, 2) Towards Combating Discontinuities of Intergroup Relations in American Society at Mizzou through Education, 3) Required or Optional Racial Awareness Curriculum to Elicit Institutional Reforms, and 4) Perceptions about the Institutional Response / Curricular Reform Efforts to Change Mizzou's Campus Racial Climate. 


\section{Chapter Five}

\section{Discussion}

Earning an education and obtaining post-secondary degrees is important to the future of the economy and the workforce for those who attain them. Institutions of higher education have a responsibility to properly prepare its graduates to interface and thrive in a global society. Not solely for future economic stability or financial gain of course, but to be well-versed and equity-minded citizens who honor, respect and advocate for social justice and its reform where necessary. Graduates should be prepared to maintain good social interactions amid intergroup relations and apply equity ideals into real world situations they will encounter. Racism, class, gender, and other social identities are a constant part of our life-worlds and influence the spaces we frequent daily. Some Predominantly White Institutions across the nation continue to struggle with ways to understand how to mitigate or prevent such racially charged incidents but perhaps, are missing the awareness of how to do so effectively. We are limited in our knowledge of the ways in which these tensions are mediated by faculty and administration from protests to demands and from demands to interventions and beyond. We often see or read about such major issues with campus climates but lack a wealth of knowledge on their aftermath and the perceptions of participants who can enlighten the field about the mistakes, the successes and the processes that might lead to some form of change in oppressive structures and social inequities. This study fills a gap in the literature by bringing to surface the perceptions and responses of the students and faculty-administrators after campus unrest, understanding the process of the student's demands as interventions for implementation, and by coming to understand the ways in which their demand for a required racial awareness and inclusion curriculum was navigated. After conducting the research using semi-structured interviews and document analysis instruments to obtain relevant data to understand and advance knowledge related to the phenomena of this case, the analysis of participant responses was conducted within four unique themes emergent from the completion of the data coding process. The four themes chosen to organize the data sources' responses are: 1) Process of Curricular 
Reform Implementation, 2) Towards Combating Discontinuities of Intergroup Relations in American Society at Mizzou through Education, 3) Required or Optional Racial Awareness Curriculum to Elicit Institutional Reforms, and 4) Perceptions about the Institutional Response / Curricular Reform Efforts to Change Mizzou's Campus Racial Climate. In this chapter, I have discussed summaries of the responses to both research questions that guided the study and how suppose some of the available literature reviewed earlier are situated within my analysis of the findings, practical implications for higher education and a general framework of considerations model for implementing race, identity, and inclusion-focused curricular interventions, and ideas for future research.

\section{Discussion of Research Questions}

Research Question one: What are the perceptions of the process of formulating and participating in a curricular response to an exceptional campus racial climate issue at the University of Missouri?

First, it is important to note that participants considered Citizenship@Mizzou (C@M), a diversity training course required for all freshmen and transfer students, as the actual initial institutional response to the 2015 campus racial climate issues that led to national attention. The curricular intervention was requested by an interim chief diversity officer for a faculty member to implement following the faculty member's public comments suggesting a need for a comprehensive diversity training. Thus, being centrally focused on Mizzou's four core principles of Citizenship to encourage improved intergroup relations, C@M was reported to be implemented with the support of administration and without administrative pushback. Some participants did, however, report some levels of resistance or apathy from students perceived as disinterested. As an outgrowth of C@M and following the requests of students-not completely fulfilled by it-wanting a more stringent and race-focused academic experience, two faculty members collaborated to develop a faculty-led institutional response known as Race and the American Story (RAS). In summation, data sources illustrate that the process of implementing both Citizenship@Mizzou and Race and the 
American Story was fairly smooth and welcomed by a large portion of university faculty, administrators, and students. Participants, who helped develop RAS were representatives of Black Studies, Political Science/Kinder Institute, Music, and History and reported that each department granted swift support and approval for the interdisciplinary collaborative course. The RAS faculty planning team also reported having no real dissention amid colleagues in determining course content for the syllabus; instead, they each presented content reflective of their own discipline expertise, to expose students more effectively to familiar and unfamiliar content not traditionally examined with a Critical race lens in a historical context.

There are several things to note when using the two frameworks that guided this study, Critical Race Theory/Intersectionality and Bronfenbrenner's Human Ecological Systems theories, to drive discussion. Much of the literature reviewed in Chapter Two fit, in some way, under the domain of these two frameworks and can be used to tease out the overarching tenants and assumptions derived from two frameworks to discuss both research questions. First, let us be reminded of what led to the protest of 2015 at Mizzou. CRT advocates for the examination of race/social categories within their historical context to capture their impact on American systems and vice versa. Consider the long history of racism and oppression felt by Mizzou's Black students for many years; of which, prompted the need for The Legion of Black Collegians to put forth demands for the betterment of the Black community in 1969. More than 45 years later, Black students sought the need to form a coalition called Concerned Student 1950, named in honor of the year the first Black student was admitted to Mizzou. But also imagine the types of oppressions and mistreatment this first Black student, along with others admitted in the early 1950s and beyond experienced. These oppressions and mistreatment remained a constant at Mizzou and led to Black students demanding an improved campus racial climate nearly 20 years following the admission of its first Black student. The ongoing discontinuities in race relations over time speak to the notion of a permanence of racism that continues to plague American citizens, nurture inequitable systems and create tensions within the human ecological system. Throughout the years, Black students at Mizzou felt it necessary to 
raise their concerns with the university's administration; often being prompted by recent acts of racism and discrimination on campus and the unjust murder of Michael Brown at the hands of White police. All things compounded; it was the protest of 2015 that produced enough national attention to highlight Mizzou's ongoing campus racial unrest. The Concerned Student 1950 coalition perceived their university administrator's responses to their occasional mistreatment and discomfort, as dismissive and inadequate to remedy tensions amid their human ecological system. As national attention across America grew, colleges and universities launched a national campaign to stand in solidarity with Mizzou using the hashtag \#Istandwithmizzou. According to Ndemanu (2017) students across the nation advocated for change at Mizzou but also began to reflect on their own institution's campus racial climate and intentional supports or lack thereof, prompting Black students from more than 70 institutions to put forth demands to their school officials; the number of institutions has continued to grow since 2017. Administrators across the higher education spectrum rallied together with their senior leadership and university constituents to assess their perceived threats to similar negative national attention as Mizzou and how they could be proactive to avoid succumbing from any unchecked and rampant acts of racism and discrimination. Thus, it was these occurrences that led to several organizational changes within Mizzou's administrative ranks; including the resignations of both the system president and campus chancellor and the hiring of a chief diversity officer to lead a new Division of Inclusion, Diversity, and Equity. With looming tensions amid the social interactions and the students demand for immediate action, Dr. Shonekan pulled a trusted group of students and faculty together to implement Mizzou's first institutional curricular reform intervention, Citizenship@Mizzou, in response to the students' demands. After an analysis of the findings, I found a bit of interest convergence and a critique on liberalism to have been present during the journey to implementation. Specifically, I infer that the swift nature of the creation and implementation of C@M was in - in part-in the best interest and benefit of Mizzou's reputation and their need to coil the narrative and calm the racial unrest that unexpectedly went viral. However, I believe that Dr. Shonekan's efforts were 
very genuine, and her intentions were to begin correcting a very fractured campus climate, but she realized the reality that certain words, like diversity, would be off-putting to some. Thus, she sought to find a way around the resistance to reform by offering a title that might limit any resistance from the onset of the mention of a required "diversity training." Additionally, while not a curricular intervention, the football teams historic boycott to playing anymore games until the demands were met amplified the significant financial loss that would follow if demands were not addressed. The football team's participation in the protest, undoubtedly bolstered the voices of Concerned Student 1950 and prompted administrators to take swift action. While many of the participants of this study were happy to have C@M as some form of an intervention to address the discontinuities in intergroup race relations, they did not think it was an adequate response to the campus racial climate issues plaguing the social interactions within their microsystems. In fact, some students blamed administration for tying the hands of Dr. Shonekan by not allowing the training course to directly address race and racism; thus, stating that C@M was essentially a band-aid or offered just to say that Mizzou had something in response to what the students demanded. Such perceptions are in alignment with CRT's assumption of a Critique on Liberalism; whereby, change happens slowly and moderately to ensure that the dominant population in power do not become angered and see the change as palatable, but at the expense of those who are oppressed and demanding change. Some of the students complained that White peers sat in the back of the auditorium, did not appear interested or engaged and in some cases were resistant to paying attention to individuals from vulnerable populations, who shared their lived experiences and perspectives. Such superior dispositions are congruent with the assumption of whiteness as property; wherefore, White people see non-White people as inherently inferior and thus, are disinterested in what goes against what has been deemed as standard White American intellectual property. Consequently, when intellectual property and their narrative is controlled by those is positions of power, who determine who has the right to certain belief systems and what will be excluded, it perpetuates a permanence of racism in American systems. 
On the other hand, participants in this study reported they perceived some students, including their White peers to have been influenced and more willing to embrace or engage in race/identity-focused conversations with diverse people inside and outside the training course. Some students even offered to participate as one of the band members of the Talking Drum band that played the music that prompted discussion around lyrics.

Participants who enjoyed C@M and believed in its mission, made it abundantly clear they wanted more than the two-hour training course and requested ways they can advance their knowledge of race and identity dynamics in a more direct manner. Dr. Shonekan and Dr. Adam Seagrave felt the same way as their students and joined together in total agreement to begin the development of new course dedicated to understanding the story of race in American history. But in designing both curricular interventions, I considered the literature on reframing organizations to understand the nature of relationships and interactions that occurred to successfully implement Citizenship/race-focused curricular reform interventions with ease. I was also reminded of Dr. Adrianna Kezar's work on how colleges change to frame what took place. It was an interdisciplinary group of faculty who planned Race and the American Story, which is important to note given the competitive nature of the academy that might metaphorically operate like a jungle, where power and conflict are commonplace regarding some disciplines being considered more privileged, rigorous or scholarly than others. Bolman and Deal refer to such organizational politics as the political frame. On the contrary, Dr. Shonekan and Dr. Seagrave were able to unite, despite differences in disciplinary expertise to bring together more faculty with varying expertise to unify around a common transformational goal to bring understanding and healing to a fractured campus climate. Such approach is more in line with the human resource frame, whereby it operates more like a family and centralizes needs, skills, and relationships while creating an image of empowerment for those involved. The student and faculty participants in this study remarked about the important role that each faculty member's academic discipline or lived experiences played in adding to the success of both interventions. Bolman and Deal's symbolic frame 
centralizes culture, meaning, ritual, stories and heroic inspiration and operates like a theater. Citizenship@Mizzou sought to hold high the institution's core values as a symbol of Mizzou's culture and what it means to be a good citizen at Mizzou by giving meaning to their four core values through the stories of those facilitating. Similarly, Race and the American Story did a deep dive into the historical past to examine the symbolism of foundational documents, practices, systems, and stories to understand how race and racism was ritualized throughout American history. Additionally, the faculty's organization of the interventions resembled aspects of a factory or machine found in the structural frame, which centralizes rules, roles, policies, goals, technology, and environment to shape the social architecture of both interventions. Examples would include standardizing the syllabus and the pedagogical use of intergroup dialogue to elicit a change-oriented social architecture for RAS and mandating diversity training to targeted mass audiences and offering the same structural format to each audience while using music to shape the social environment in C@M. Thus, I infer there was a multi-frame approach to reframing the operation of an academic area between the departments involved. Kezar's work on how colleges change proclaims that change frequently includes risk-taking but can be less perilous with a collective approach; I would argue that the faculty involved took a collective risk to offer a course about America's dark past and present indiscretions with race and racism to produce change.

To summarize the findings for research question one, I have listed several culminating ideas under some of CRT/I's assumptions. The assumptions I grouped together for this summary include, the investigation of relevant social categories within a historical context, how nuanced lived experiences are influences by social structures and how power is challenged. Thus, here are some important take-aways that help to bring a summative understanding of research question one. In summation:

- The ongoing discontinuities in race relations over time speak to the notion of a permanence of racism that continues to plague American citizens, nurture inequitable systems, and create tensions within the human ecological system at Mizzou. 
- The institutional responses prior to protests were perceived as dismissive and inadequate to remedy tensions amid their human ecological system. Essentially many of the students' microsystems were being attacked. (Res. Hall, student club meetings, homecoming events etc.)

- So, the historical legacies of oppression and current racism at Mizzou coupled with the murder of Michael Brown in Ferguson became the impetus for students' protests and demands, which provided the evidence for the creation of C@M, followed by RAS.

- "The Talking Drum" musical component to C@M was engaging because they examined various social categories and paid attention to the treatment of vulnerable voices through song lyrics. Students found this to be a useful pedagogical strategy to discuss controversial social issues over time through the musical expression of various well-known American artists across decades.

- RAS placed race and the intersection of identities at the center of its focus to examine over time through foundational American documents. This was reported as one of the most compelling and powerful components of the course.

The other CRT tenants and intersectionality assumptions I grouped for the next set of summative ideas, are interest convergence, critique of liberalism and intersectionality. In summation:

- Interest convergence was discovered because of the swift creation of C@M and the need to coil the narrative and calm racial tensions that went viral, but participants perceptions reported that it lacked a focus on race and racism to be a sufficient institutional response to the campus unrest.

- Interest convergence was also discovered because the football team's historic boycott amplified a potential significant financial loss that would have cost Mizzou millions of dollars in forfeitures if they did not play in an upcoming game. The football team's boycott bolstered the voice of Concerned Student 1950 that led the protest. I also note the intersecting identities of being an 
athlete, Black, and Male. In other words, their athletic identity placed them in a position to challenge power.

- A critique of liberalism was discovered because participants felt that there was a heavy burden of racial equity work being placed primarily on one faculty person who identifies as both Black and a woman, which under CRT/I analytical assumptions, places her in a difficult position, being from two traditionally oppressed identities, to navigate such a challenging role as a change agent for intuitional reform.

- A critique of Liberalism was also considered to be present given the name change of the diversity training course from its original name, Diversity@Mizzou to Citizenship@Mizzou. While the purpose of this curricular reform was not interpreted by any of the participants or me as the researcher, as being created by Dr. Shonekan to be disingenuous or to only appease White people, I do consider the removal of the word "diversity" from the name-at the request of a student-to be a critique on liberalism. Primarily because doing so makes it more palatable for those in privileged positions and is less likely to anger them, but at the expense of those demanding explicit reform; presumably making change come at a slower pace. Additionally, removing the word "diversity" from the title of the training course is not a stand-alone aspect, it had implications for the content and nature of how explicit the training would or would not be. This was evident through participant responses, who were desirous of a training more explicitly focused on race and racism. I believe that Dr. Shonekan's efforts were very genuine, and her intentions were to begin correcting a very fractured campus climate, but she realized the reality that certain words, like diversity, would be off-putting to some.

For whiteness as property, we should note:

- Because some White students were reported to be disinterested and disengaged during the presenters sharing of lived experiences, it perpetuates a permanence of racism. Thus, it brings 
attention to their supposed right to a disposition of superiority, the right to exclusion and a resistance to challenge their established understanding of Whiteness as a form of already attained intellectual property.

Research Question two: What are the perceptions of how this curricular response addressed discontinues in race and racism on campus and contributed to organizational change and institutional reform at the University of Missouri?

The results of the analysis indicate a mix of very strong feelings towards requiring or making the curricular interventions optional. For C@M, four of the four participants involved reported that it should be required. For RAS, two of the five participants involved reported that it should be optional, while the remaining three participants reported it should be required. The opinions about requiring race-focused content are a part of the necessary data needed for senior administrators to determine the best path towards institutional reform, as it sets the tone for shaping how the institutional culture is perceived in the plight for social justice and an improved campus racial climate.

An analysis of the perceptions on how the curricular interventions addressed the existing discontinuities with race and racism and if they contributed to change, revealed mixed opinions from participants on their effectiveness. For C@M, three of the four participants involved, reported it was somewhat effective for a moderate portion of participants but needed a stricter focus on race dynamics and one person reported it "not really effective at all;" offering a 2.53 on a hypothetical scale of 1-10, where 10 is highly effective at achieving the overarching curricular reform mission in response to the 2015 protest. For RAS, five of the five participants involved reported positive perceptions about the course's ability to elicit change in a variety of ways individually and institutionally. Albeit there was much consensus around the value and effectiveness of RAS, a portion of participants desired additions to the course. For example, some desired that the course be three-credit hours instead of one [This change occurred eventually], more days a 
week, and accessible to more students. Additionally, some were unwilling to let Mizzou senior leaders off the hook with either of the two curricular interventions by themselves, as adequate responses to the events of the case. In fact, this portion of participants perceived the implementation of RAS as taking too long to be made available but to no fault of the professors who designed it. One participant even referenced student policing and campus structures symbolizing racism needing to be addressed beyond the notion of curricular reforms. Overall, participants of this study provided very thick and rich data for analysis, particularly around their appreciation and astonishment with their exposure to course content and intergroup dialogues, where they examined race and racism in America, within a historical context through the racial unrest at Mizzou. Their examination of America's founding documents over time with a Critical race lens, allowed students to understand and embrace new ideologies and behaviors, consistent with the overarching mission to improve intergroup relations and remedy a tense campus racial climate at Mizzou.

I reflected upon the ways in which aspects of identity development and intergroup dialogue surfaced either directly or indirectly, especially in RAS. For example, let us revisit a portion of the literature on identity development I referenced, in Chapter Two, namely, the Individual Diversity Development Theory to conjoin it with how I surmise it to have been applied in the context of Race and the American Story. Chavez, GuidoDiBrito, and Mallory (2003) created Individual Diversity Development (IDD) as a framework to gauge ones' process of development in the following areas: cognitive, affective, and behavioral when considering how people value the complex differences in others and themselves. This framework specifies six non-linear dimensions in which developmental movement occurs, which include: 1.) Unawareness, 2.) dual awareness, 3.) questioning and self-exploration, 4.) and 5.) risk taking or other exploration, and 6.) integration and validations of others. To determine ones' achieved dimension across diversity as a whole, it is measured by looking at a person's a.) cognitive, b.) affective and c.) behavioral condition (Evans et. al, 2010; Chavez et al., 2003). First, Unawareness/lack of exposure to the other represents the place where people are when they have little to no knowledge or exposure with others who are unfamiliar. Cognitively, there is no 
consciousness or exposure to difference; affectively, there are no reference points of experience which yields a void of any type of feeling for otherness; behaviorally, the individual does not act when presented with differences. Next, in Dualistic awareness, cognitively people understand difference dichotomously without reflecting on it and thus, interpreting others from sudden analyses. Affectively, the egocentric self does not question their own analyses or expectations about "the other," making themselves superior. Behaviorally, the individual may have limited contact with others outside of their familiar groups, causing the person to correct others by pointing out what they perceive as incorrect behavior or by trying to exclude or eradicate others from the environment (Chavez et al., 2003). The behavior would be less likely to change if not presented with an opportunity or intervention - like Intergroup Dialogue - that could lead to changed feelings (affective) as a result of examining inaccurate information (cognitive). We know from the findings, that deep discussion/dialogue was consistently reported as a vital pedagogical strategy to create meaning and advance knowledge in-for some-unchartered territory. The literature also showed similar value in measuring the cognitive and the affective positions of the learner; Nagda et. al (2014) discovered issues with the way some faculty had previously structured their diversity courses. Thus, they suggested to faculty that when teaching about diversity and oppression, it needs to address students' cognitive and affective development issues. They recommended utilizing both didactic and experiential learning while also motivating students towards both intellectual and emotional responses to course content (Nagda et. al, 2014). In response to these recommendations, I consider Intergroup Dialogue to be an ideal curricularintervention strategy to intentionally aim toward achieving optimal outcomes. The unification of the IDD theoretical framework paired with RAS in practice, captures the essence of how people can be positioned for social change and bolster the kind of equity-mindedness needed to improve intergroup relations and dismantle institutional systems of oppression.

To achieve the desired transformational institutional reforms to remedy campus unrest requires administrators and faculty to offer a direct approach when combating racism and discrimination. I concluded 
that Race and the American story pushed back on the notion of Whiteness as Property towards achieving this end. A CRT/I analysis underscored my awareness of the Permanence of Racism that exists by coming to understand how students examined the treatment of race and how it was used to oppress people and create systems of oppression within a historical context and today. The intergroup faculty of RAS afforded all of their students to the intellectual property, lived-experiences and counter-stories of Black voices, that have been excluded from mainstream curriculum and educational institutions. It pushed back on idle White supremacy, White normalcy and White superiority which reinforces the oppressive ideology that black people are inherently inferior to White people. The course combated the discriminatory rhetoric, ideologies and dispositions taught, learned and upheld from generation to generation; substantiating CRT's assumption that there is and will always be a Permanence of Racism in America, of which we must constantly resist, challenge and fight against with swords of equity. Moreover, I was able to obtain at least one course evaluation document for RAS, which revealed very favorable responses from 15 students in one section of the course during the spring of 2018. In terms of teaching delivery, 93\% of the students strongly agreed the instructor was knowledgeable and enthusiastic about the topic, $73 \%$ strongly agreed and $20 \%$ agreed the instructor effectively used examples/illustrations to promote learning, 93\% strongly agreed and $7 \%$ agreed the instructor fostered questions and/or class participation, and 67\% strongly agreed and 33\% agreed that the instructor effectively used teaching methods appropriate to the course (e.g., critiques, discussion, demonstrations, group work). As for the learning environment, $80 \%$ strongly agreed and $20 \%$ agreed the instructor stimulated student thinking and learning, $87 \%$ strongly agreed and $7 \%$ agreed the instructor promoted an atmosphere of mutual respect, regarding diversity in student demographics and viewpoints, such as race, gender, or politics, and only three students remained neutral and one student strongly disagreed. Analytically, I considered these favorable results to reflect the instructor's positive treatment of vulnerable voices, perspectives and experiences of all students. Additionally, results were overwhelmingly favorable regarding student's recommendations for their peers contemplating enrollment in several areas. 
For example, $93 \%$ recommended the class for its content and for its positive learning environment and $87 \%$ recommended the course for the class structure and because of the instructor's teaching style. Approximately six students remained neutral, disagreed or strongly disagreed with aspects of their experience in this particular section of RAS, but by and large the course was well received. However, I was unable to determine the racial or social demographics for any of the students who participated in the course evaluation; I think it would be helpful for instructors and their supervisors to consider such demographics to determine which demographic(s) of students they need to make a concentrated effort to improve student perceptions about their experience. This strategy is considered an equity-minded approach because it does not automatically blame the students for their experience in the course, but rather forces the faculty to be reflexive and adjust their future methods.

To summarize the findings for research question two, I have listed several culminating ideas under some of CRT/I's assumptions. The first set of assumptions I grouped together for this summary include, identifying race as a relevant social category within the historical context (time) of how it operates in social structures, how power is challenged and counter storytelling. Thus, here are some important take-aways that help to bring a summative understanding of research question two. In summation:

- The inclusion of American foundational documents (The constitution and Articles of Confederation, the Bill of Rights, Jefferson's letters and early constitution draft, Declaration of Independence etc.) allowed students to examine them through a Critical Race lens within a historical context to consider how social structures have influenced the idea of race and the mistreatment of those who have traditionally been oppressed.

- Counter storytelling and paying attention to the vulnerable voices from the works of Black authors and scholars, who offer counter narratives to cast doubt on the validity of accepted premises or 
myths held my dominant groups. It allowed participants access to intellectual property that exposes, and critiques normalized dialogues that perpetuate stereotypes of non-dominant groups.

The next group of CRT tenants to note ideas is a push back on Whiteness as Property and the Permanence of Racism. In summation, we should note:

- To achieve the desired transformational institutional reforms and change the campus climate, participants wanted an explicit focus on race to combat racism and discrimination.

- The interdisciplinary faculty's pedagogical strategies and course content pushed back on idle White supremacy, White normalcy and White superiority which reinforces the oppressive ideology that Black people are inherently inferior to White people.

- It combated the discriminatory rhetoric, ideologies and dispositions taught, learned, and upheld from generation to generation; substantiating CRT's assumption that there is and will always be a Permanence of Racism in America.

\section{Practical Implications for Higher Education and A General Framework of Considerations Model for Implementing Race, Identity and Inclusion-focused Curricular Interventions}

Institutions of higher education remain focused on retention of its students for any number of reasons associated with success in a variety of areas that benefit their continued operation and reputation to the public. Likewise, many institutions over the last decade or more have touted their aspiration to or success with being "diverse," welcoming of "diverse students," "embracing diversity," and have amped up a slew of diversity statements for each department on the campus. Some institutions have even engaged in organizational change strategies by increasing diversity, equity, and inclusion personnel to support or become change agents who work on institutional reform efforts proactively or reactively. While pervasive racism and inequitable policies run rampant amid our college campuses, we might be hard pressed to find one desirous of recognition as a breeding ground for the mistreatment of racially and socially diverse 
students and upholding oppressive structures. However, some institutions remain silent, slow, minimally focused or advocacy driven to produce meaningful, significant, and large-spread change to their campus' human ecologies. On the other hand, there are institutions officials who remain adamant about improving their campus' racial climate and either healing or shielding it from racism, discrimination and inequitable policies and practices. Either way, the students, and families, who are vital stakeholders to the economic sustainability of colleges and universities have a huge impact on their reputation and forward progression, as it pertains to students' overall academic and social experiences. We know from the literature and the counter-stories of underrepresented individuals, that many of these students continue to feel marginalized, unwelcomed, unsupported by faculty and administrators, misunderstood by peers from privileged groups and are in demand of an improved campus racial climate to aid their academic achievement and student development. But how concerned are faculty and administrators about these claims? And how inclined are they to actively make pivotal changes to their institution's organizational structure, policies, practices, and curriculum as it relates to equity, inclusion, social justice, and diversity?

\section{Higher Education Senior Leaders and University Staff/Administrators}

Campus racial unrest can no longer be ignored or temporarily placated as new episodes of racism and discrimination air, because more public outcry and protests for racial equity have surfaced outside of the Mizzou case since 2015 and, I believe, will continue. For example, protests have erupted after being sparked by instances of systemic racism, homophobia, transphobia, historically oppressive monuments, hate-speech disguised as free-speech, more unjust killings of Black bodies at the hands of White police, resistance to the Black Lives Matter movement, and much more. College/university leaders today, should engulf themselves in understanding the full scope of each of the campus protest cases in recent years to guide their decision making and administrative actions. The nuanced stories of each institution's campus racial unrest, under a Critical race/intersectionality analysis, can serve as a resource to understand how to effectively begin engaging campus constituents in executing a collaborative and equity-minded 
institutional reform plan. Furthermore, I urge college/university officials at all levels, who may not have experienced said protests and campus racial climate issues, not to assume it will not happen on their campuses because it may only be a matter of time or one incident away from happening. To underscore this idea, consider some of the institutions that have been forced to grapple with such campus unrest, which include but are not limited to: University of California Berkley, University of Wisconsin, Syracuse University, Stony Brook University, Kent State University, Northern Kentucky University, University of Oklahoma, University of Oregon, and Claremont McKenna College. Higher education senior leaders and university staff/administrators would benefit heavily from acting now to capture the pulse of their entire campus and local communities to prepare themselves for remedying what has already happened or preventing what may inevitably be approaching. But let me be clear in my assumptions for implications, higher education leaders should not solely engage in this work to divert, remedy, or combat the ills of racism, oppression, discrimination or inequitable systems. Leaders should participate in transformational institutional reform because they have a moral responsibility to protect students from harm and afford them an atmosphere conducive to their academic achievement and social development, as it is morally right to do so. In doing so, I contend that such development cannot be done so with a homogenous or onesize-fits-all approach under the guise of equal-opportunity-for-all, but rather with intentionality by ensuring that each student is afforded what they need to be successful. Black Students, indigenous students, students of color, LGBTQ+, and students with varying abilities must be given and/or a part of systems that ensure they, at a minimum, have what is necessary to see what others see, feel what others feel, do what others do, and have access to what others have access to; without struggle and without having to create the necessary supports, initiatives, and programs for themselves. Leaders must work proactively to dismantle inequitable systems and lessen the likelihood for institutional racism to take up residence and permeate policies and practices, elevate the need to be equity-minded, and promote inclusivity. It is the job of university officials and faculty to prepare students to be model citizens who 
embrace humanity in all its intersecting identities, to be just, to walk uprightly and to advocate for social justice and equity in all manner of life.

\section{Faculty, Deans, Department Chairs, Curriculum Committees, Human Resources and Hiring committees}

Organizational change and institutional reform do not solely rest upon the shoulders of senior leaders/administrators. Higher education institutions are, in fact, businesses but must remember their commitment to house faculty concerned with making meaning, enlightenment, the advancement of knowledge and preparing students to be equity-minded global citizens who represent the name of their institution with the utmost dignity. Much of these ideals fall within the domain of the professoriate, therefore, I would argue that it is incumbent upon deans, human resource departments and hiring committees to due diligence in hiring faculty with attentiveness, as to ensure their commitment to creating explicit formal educational opportunities for their students around race and identity and diversity, equity, and inclusion. The hiring practices and process should require candidates to respond and directly explain how they would both infuse said ideals into the curriculum but also how they would create or teach curriculum that intensively addresses race, identity, diversity, equity and inclusion. Deans and department chairs and fellow faculty may consider creating interdisciplinary faculty committees aimed at diversifying the curriculum and advancing faculties' Critical consciousness, and knowledge of how race, identity, diversity, equity, and inclusion are or can be situated within their own discipline. Similarly, while not meant to be a faculty-resource/development committee, the interdisciplinary faculty team that created Race and the American Story in the Mizzou case is a perfect example of an interdisciplinary faculty collaboration around curriculum development. It would behoove faculty and their supervising leadership to ponder about the kind of student they hope to produce and the kind of student that will represent their degree program, department, college, or university itself. They can benefit from answering these questions and structuring their curricula to produce professionals who have explored the story of race in America and their discipline, who have engaged with peers in an intergroup relations dialogue about 
power and oppression, who have explored their individual diversity development and who will possess an equity-mind within society. Deans, department chairs and faculty who fail to embrace and incorporate the aforementioned ideals and controversial subject matter into their academic programs and classrooms, are robbing their students of the holistic development and intellectual dialogue necessary to aid their ability to be equity-minded and advocacy driven for social justice in our American institutions where they will work. Thus, upholding the notion of Whiteness as property, where the education system is controlled and reflective of White standards of normalcy. Likewise, the failure to explicitly diversify and reform our curriculum reinforces the permanence of racism, inequity, and oppressive systems when our students enter the workforce as, for example, medical providers, attorneys, law-enforcement, educators, policymakers, legislators, politicians, hiring committee members, book publishing executives, filmmakers, executives who green-light films and television productions, musicians etc. All of which are professions where we know systemic racism and discrimination are pervasive, but if we grab control of the education system and curriculum that we offer our students and make it more equity-based and inclusive, we can begin to dismantle aspects of institutional racism and discrimination from our American systems, policies and practices. Accreditation agencies and government funding agencies would be better serving if institutions are mandated, more explicitly, to display how their institutions are working to dismantle systemic racism and discrimination within and beyond the curricular offerings. Based on the responses and literature examined throughout this study, I have proposed a framework of considerations for higher education practitioners/educators in general as a resource towards implementing a race and identityfocused inclusive curriculum.

The framework I propose is not a detailed or ridged model with step-by-step levels or linear statuses, but rather a framework of considerations. The utility of this framework is for higher education practitioners/educators to use as a reference of things to consider when contemplating or planning to implement a race and identity-focused curriculum under the guise of institutional reform and cultivating 
inclusive excellence at their institution. This framework is meant to be a working model that evolves with time and additional research; thus, is not an exhaustive list of considerations for conducting this work but it reflects the summation and my interpretations of the data findings in this study. After crafting a draft of the framework from what I extrapolated and interpreted from data, the draft was given to the faculty participants, who possess the scholarly and experiential knowledge necessary to critique the framework for potential effectiveness amongst higher education professionals. After receiving their feedback to the draft, modifications were made to more closely align with the recommendations and considerations of faculty who experienced implementing a race and identity-focused course and/or diversity training course into their institution's curriculum.

\section{Towards Implementing a Race and Identity-Focused Inclusive Curriculum (TIRIFIC):}

\section{Framework of Considerations}

Figure 6 is a condensed graphic display of the full version of the framework included in figure 7 I created as a part of my contribution to the field of higher education. 
Figure 6

\section{Towards Implementing a Race and Identity-Focused Inclusive Curriculum: Framework of Considerations}

T.I.R.I.F.I.C. Developed by Bruce E. Mitchell II

\section{A Backwards look: Outcomes to Preparation}

05

A 365 Approach Towards Cultivating

Equity-minded Curricular Reform

Developing Inclusive Excellence-365: Professiona

Development Academy

Offer rigorous and ongoing instructional strategies training (ens throughout the yhing faculty, department chairs an development academy for inclusive excellence.

\section{3}

Impact on Degree Programs

- Require an adequate number of both required and optional opportunities for foundational and advanced diversity education.

For Teacher Preparation programs, prepare future

teachers to:

- Infuse and/or intensify courses with ideals of diversity,

equity, inclusion-connect aspects of race, racism, and

- Avoid a sole focus on slavery-address the counter-stor

of slavery and reconstruction

address the historical, rich, and pioneering contributions

of vulnerable groups

Use a Critical race andintersectionality lens to examine the traditional White standardized education and

Branding \& Marketing:

to market and increase awareness of diversity education course offerings and normalize during academic advising session

\section{1}

Toward Implementation

Research, Evaluation, and Planning:

- Institutional research partnership

- Administer campus racial climate survey

- Conduce needs, desires, and demands assessment

- Courses/trainings

- Create a diverse Development and Management

Coalition for Diversity Education Curriculum

Secure strong institutional:

- Endorsement

: Institutional authority
04

The Course/ Curriculum

Organizational and Pedagogical strategies:

- Synchronized course syllabi

- Students can visit multiple sections of the same

course taught by others

- Racialy \& sociang duerse readings and assignments

t social categories

within historical context-Intergroup dialogue

student engagemen

- Positive dispositions to course content-Exposure to

multiple perspectives and underrepresented voices

- Universal references: arts, music, film, famous speeches/fgures, documents-explicit focus on race

Pssionate and Prepared instructors:

- Critical race and identity analysis and dialogue to

: Collegial relationships - dentity-related litersed in the diversity of raceriences tough or competing ideologies

\section{2}

on all Institutional

Constituents

cular and Development

Opportunities and Policies
Require at least one foundational and one advanced diversity education opportunity that explicitly focuses on race, identity, diversity, equity and inclusion for

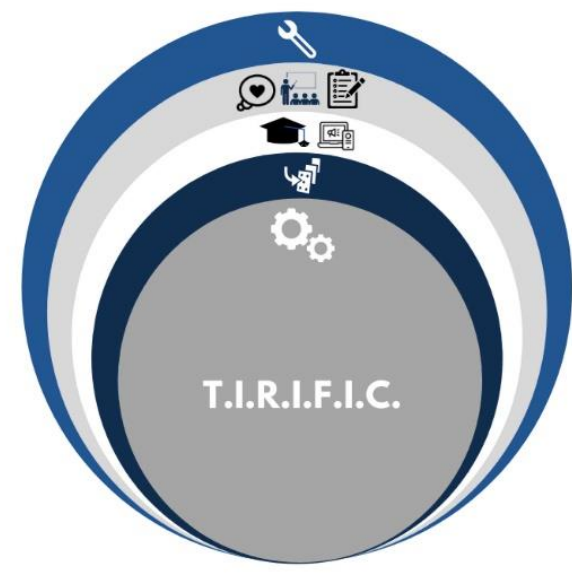


Figure 7

Towards Implementing a Race and Identity-Focused Inclusive Curriculum: Framework of Considerations T.I.R.I.F.I.C. -Full Version

\begin{tabular}{l} 
Towards Implementing a Race and Identity-Focused Inclusive Curriculum: Framework of Considerations \\
T.I.R.I.F.I.C. \\
Developed by Bruce E. Mitchell II / See graphic model for a condensed display \\
$\qquad$ A Backwards look: OUTCOMES TO PREPARATION \\
\hline A 365 APPROACH TOWARDS CULTIVATING EQUITY-MINDED CIRRICULAR REFORM \\
\hline $\begin{array}{l}\text { Developing Inclusive Excellence-365: Professional Development Academy } \\
\text { year as a part of a professional development academy for inclusive excellence. }\end{array}$ \\
\hline THE COURSE / CURRICULUM \\
\hline
\end{tabular}

1. Organizational and Pedagogical strategies:

a. Create collaborative interdisciplinary faculty teaching teams

b. Develop collaborative and synchronized course syllabi

c. Allow students to visit other sections of the same course to diversify their experience and gain exposure to additional perspectives.

d. Ensure readings and assignments are diverse and reflective of scholars and individuals with varying and intersecting identities. (e.g., women who also identify as Black)

e. Identify and examine relevant social categories within a historical context to understand the story of race and identity in America as it pertains to e.g., foundational documents, institutional practices, policies, laws or the major discipline being studied by the student.

f. Use intergroup relations dialogue as often as possible

g. Make all attendees feel comfortable speaking

h. Never allow students to be disengaged and dismissive of content

i. Create an atmosphere that advocates for an exposure to multiple perspectives and highlights the voices, counterstories, experiences, and perspectives of people from traditionally oppressed groups.

j. Consider using universal references to frame the subject under analysis or initiate course dialogue (e.g., music, arts, film, famous speeches/figures)

k. Place an explicit focus on race, racism, identity, and equity in classes where applicable, ensure courses are racially and socially diverse. 
Figure 7 (Continued)

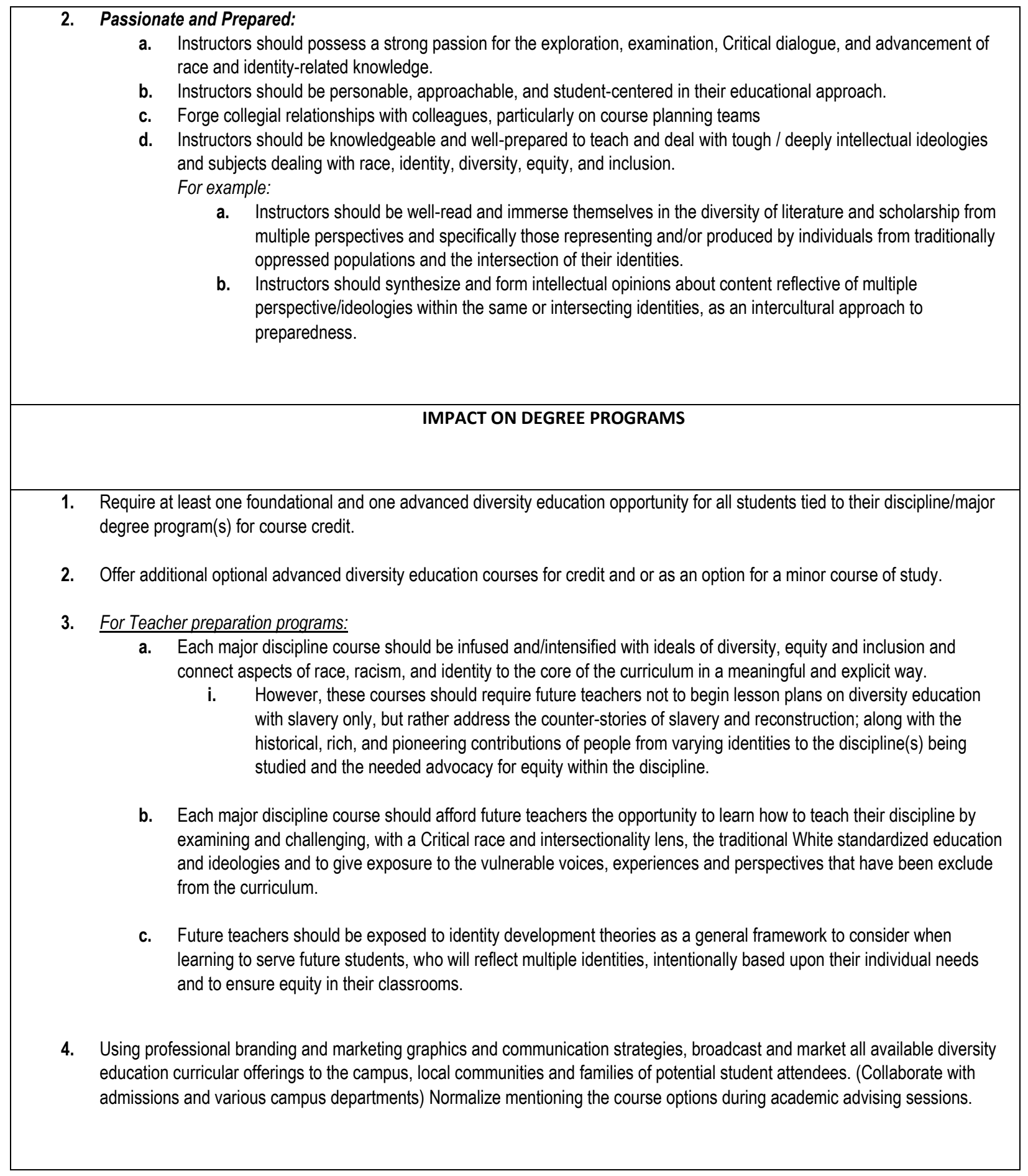


Figure 7 (Continued)

\section{IMPACT ON ALL INSTITUION CONSTITUENTS}

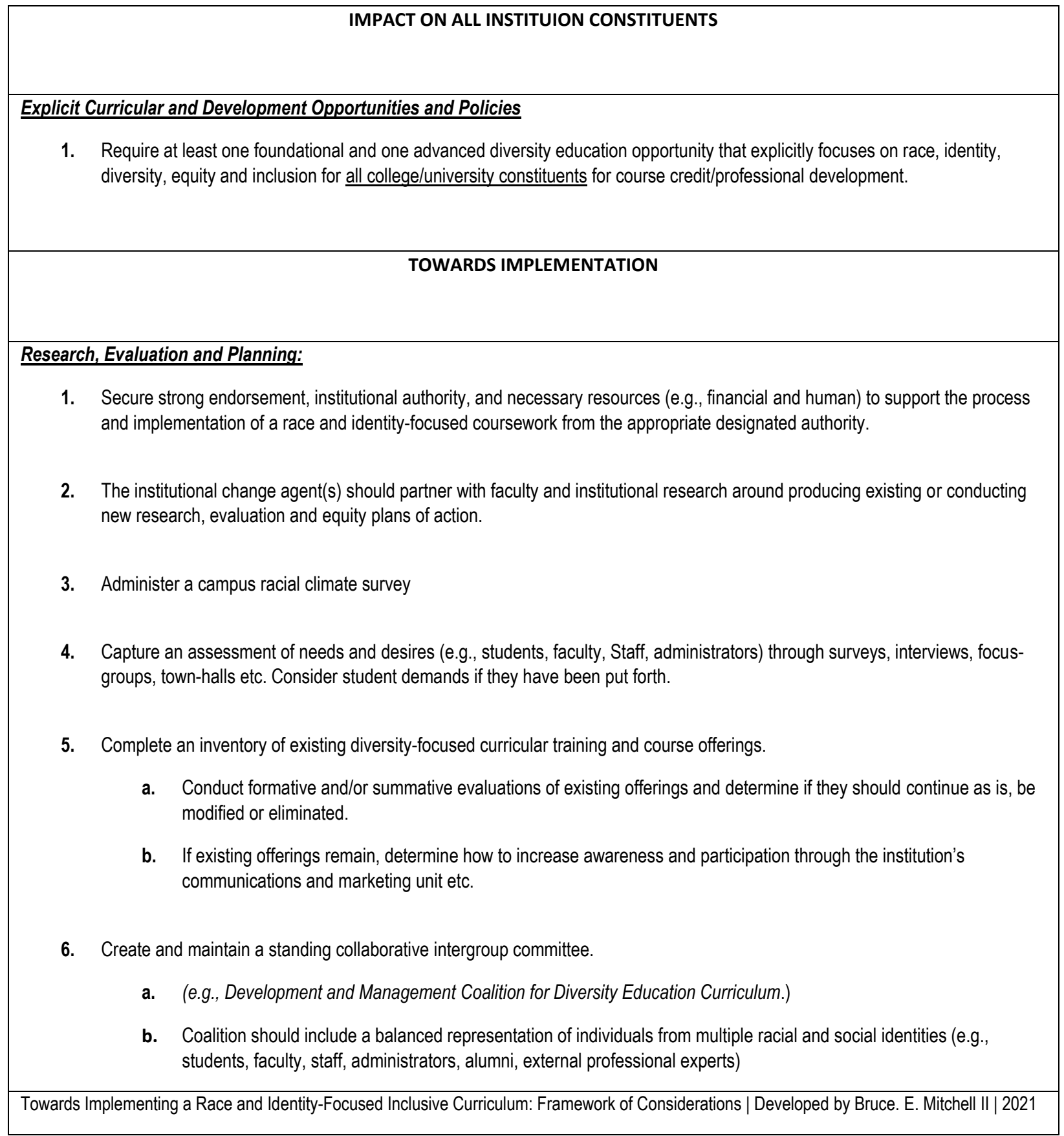

Note. Mitchell, 2021

Figure 7 is a detailed depiction of the working-framework I propose to higher education

practitioners/educators. Based on the feedback from faculty participants in this study, I use a somewhat backward design approach to listing considerations to present the potential outcomes of a 365-day approach towards cultivating equity-minded curricular reform and the details for effective 
course/curricular implementation. Thus, I listed first, the information pertaining to the development of an ongoing instructional strategies training in the form of a Developing Inclusive Excellence-365: Professional Development Academy, Organizational and Pedagogical Strategies, and considerations for Passionate and Prepared Instructors. Because they were deemed by participants as uniquely original and afford practitioners a straightforward look at the most vital aspects of what elicits a successful team of practitioners/educators developing professionally around inclusive excellence and the course/curriculum itself. The framework then moves on to the Impact on Degree Programs, Impact on All Institution Constituents and finally Towards Implementation. Arranged in this manner, higher education professionals are likely to be intrigued by having first viewed the outcomes of what their institution could offer as opportunities, before reading considerations about implementing something unexplained.

\section{Future Research}

Ongoing research in the realm of curricular reform efforts to institutionalize race-identity-focused curricula is vital to the transformational goals to create or maintain calm campus racial climates. Critical Race Theory would have us to believe that racism is permanent; however, it is how higher education leaders and faculty prepare to combat ideologies of racism and White supremacy that will determine how racially and socially inequitable policies and practices are dismantled or maintained. Likewise, their response can determine how tense or calm social interactions will be on their campuses. Instead of addressing change resulting from primarily episodic events converging the change and calming the climate at the time of crisis, we would benefit from ongoing research that academically prepares all college/university constituents to be more individually diversity developed and equity minded. My recommendations for research would be for researchers to study the existing curriculum or curricular reforms for students and professional faculty/staff around, for example, race, identity, intersectionality, diversity, equity and inclusion at colleges and universities. In some instances, institutions already have solid or developing academic approaches in place to educate their constituents around the above- 
mentioned ideals, but they are not widely known, required, or utilized. For example, The University of Missouri system offered a diversity training course for faculty and staff prior to the protests of 2015, but its existence was not well known, it was not required and thus, it was not well utilized until after the university received widespread national attention and Black students' demands for a required cultural awareness and inclusion curriculum. In fact, the training course was called Diversity 101 and prior to the protest it was voluntarily attended by approximately $12-20$ people per session; post-protests the attendance increased to 80-100 participants. The sessions were led by Dr. Marlo Goldstein Hode, an administrator responsible for Strategic Diversity Initiatives, who received a significant boost in support recreating her PowerPoint video presentations as high-quality videos. Mizzou participants received a certificate of completion, but the St. Louis campus now offers a digital badge incentive for those who complete a set of diversity related curricula, to attain as a more formalized and rigorous display of diversity training. It would be interesting for researchers to explore more about diversity training/course curricula like this and determine how to improve widespread awareness about such resources already being provided or developed.

Second, it is my intention to expand upon this research by further developing the T.I.R.I.F.I.C. Framework of considerations. I plan to continue conducting research that can produce a detailed example for consideration, of a ready-made race and identity focused inclusive curriculum that can be used as a more in-depth curriculum development resource in the process of implementation. This may include a canon of potential course titles, syllabi examples, assignments, content, etc. that can be modified by practitioners/educators across the nation, to fit their respective institutions. In like manner, it is my hope to also create a similar ready-made curriculum or set of professional/faculty development strategies and resources that can be used to implement in my proposed Developing Inclusive Excellence 365: Professional Development Academy I include in the T.I.R.I.F.I.C. Framework of considerations. Similarly, I would venture to do the same for teacher preparation programs. 
Third, I would advocate for an ethnographic study of several participants from several different and intersecting identities to observe their interactions before, during and after curricular interventions in their various microsystems. A more in-depth and participatory approach to field research using ethnography and phenomenological methods would be highly useful to inform practitioners and campus leaders about the effectiveness of race/identity-focused curricular offerings on participants' cognitive, affective and behavior motivations to bend towards equity and social justice advocacy. I believe that very rich data and thick descriptions can be gleaned from a researcher's participation in a course such as Race and the American Story that would aid practitioners in their decision-making to either continue, modify, advance, or change the course of action with existing approaches.

Fourth, I believe that further research can be conducted on Race in the American Story as a "project course" that has expanded to nearly 10 colleges and universities nationwide and one globally. The interchange of ideologies, experiences, presentations, and perspectives amongst students from multiple colleges and universities is an astonishing phenomenon that deserves more understanding and attention because of its nationwide intergroup relations dialogue approach to addressing the story of Race throughout American History. Being able to conduct field research in both virtual video spaces and during their annual face-to-face symposiums would be yet another opportunity to collect very rich and meaningful data that might lead to the project's expansion across more institutions who may opt to join the multi-institution academic collaboration and allow their students and faculty to participate.

Fifth, I would also love to see a mixed methodological study conducted with an explanatory sequential design. This would allow results from a quantitative survey administered to a large number of participants to be used to inform the design of a qualitative study and determine what specifically will be observed or included in semi-structured focus-groups or interviews. This methodological approach will allow the researcher to expound further upon the data collected from the initial quantitative data collected. Given the likelihood of subjectivity and competing opinions about the nature and necessity of 
diversity curricula, it would be helpful to understand how large numbers of participants within an institution respond cognitively, affectively, and behaviorally with baseline questioning and problemposing.

Finally, I would encourage the collections of data through exit surveys, interviews, surveys or focus groups upon students' degree completion. This would allow researchers to study the perceptions of students who perhaps completed their education without the targeted diversity or race and identityfocused curricular reform efforts and the perceptions of those who did participate. Furthermore, willing individuals in either case, could be studied over time post-graduation to collect data about their level of involvement in social justice advocacy and or their utility of information learned from general diversity or race and identity-focused courses.

Overall, if we fail to educate people about racism, social justice, equity and inclusion, we are perpetuating the continuation of a system where those in power can avoid recognizing, acknowledging and checking their White privilege. Those living life without taking the time to or refusing to be educated about these historical oppressions and their pervasive existence and influence on American systems today, are nurturing a deeply rooted system of oppression designed to subjugate the vulnerable. Higher education practitioners/educators and the likes of them must remain vigilant in the pursuit of carrying out institutional reform, eradicating and replacing inequitable policies and practices with equity-minded alternatives, and ongoing transformational change that reflects the needs and desires of those from traditionally marginalized communities who desire, or demand racial equity and social justice. To do this work requires special kinds of people, who have an overwhelming level of passion and preparedness to uplift and make their communities and the spaces they enter, better than they found them through collective work and responsibility. 


\section{References}

ADL. (2019). White supremacists continue to spread hate on American campuses.

https://www.adl.org/blog/white-supremacists-continue-to-spread-hate-on-american-campuses

Alex-Assensoh, Y. M. (2018). Hiring a diversity officer is only the first step. Here are the next 7. The Chronical of Higher Education. 1-5.

https://www.chronicle.com/article/Hiring-a-Diversity-Officer-Is/243591

Allen, W. (1992). The color of success: African-American college student outcomes at predominantly White and historically Black public colleges and universities. Harvard Educational Review, 62(1), 26-45.

Atkinson, D. R., Morten, G., \& Sue, D. W. (1979). Counseling American minorities: A cross-cultural perspective. Brown \& Benchmark.

Attinasi Jr, L. C. (1989). Getting in: Mexican Americans' perceptions of university attendance and the implications for freshman year persistence. The Journal of Higher Education, 60(3), 247-277.

Armenakis, A. A., \& Bedeian, A. G. (1999). Organizational Change: A Review of Theory and Research in the 1990s. Journal of Management, 25(3), 293-315. https://doi.org/10.1177/014920639902500303

Banning, J. H. (Ed.). (1978). Campus ecology: A perspective for student affairs. NASPA Journal, National Student Personnel Association.

Banning, J. H. and Kaiser, L. (1974), an ecological perspective and model for campus design. The Personnel and Guidance Journal, 52: 370-375. doi:10.1002/j.2164-4918.1974.tb04043.x

Bennett, J.E. \& Sekaquaptewa, D. Soc Psychol Educ (2014) 17: 343. https://doi.org/10.1007/s11218-014-9253-y

Barker, R. G. (1968). Ecological psychology; concepts and methods for studying the environment of 
human behavior.

Bolman, L. G., \& Deal, T. E. (2018). Reframing Organizations: Artistry, Choice, and Leadership. John Wiley \& Sons.

Bradley, S. W. \& Garven, J. R. \& Law, W. W. \& West, J.E. (2018). “The impact of chief diversity officers on diverse faculty hiring" NBER Working Papers 24969, National Bureau of Economic Research, Inc.

Breaux, R. M. (2012). Nooses, Sheets, and Blackface: White Racial Anxiety and Black Student Presence at Six Midwest Flagship Universities, 1882-1937. In Higher Education for African Americans Before the Civil Rights Era, 1900-1964 (pp. 49-80). Routledge.

Brinkmann, S., \& Kvale, S. (2015). Interviews: Learning the craft of qualitative research interviewing (Vol. 3). Thousand Oaks, CA: Sage.

Butler, J. (2015, October). Letter to the Editor: Dear Chancellor Loftin: It isn't enough. https://www.themaneater.com/stories/opinion/dear-chancellor-loftin-it-isnt-enough

Chang, M. J. (1996). Racial diversity in higher education: Does a racially mixed student population affect educational outcomes? (Doctoral dissertation, University of California, Los Angeles).

Chavez, A. F., Guido-DiBrito, F., \& Mallory, S. (2003) Learning to value the "other": An individual framework for diversity development. Journal of Collee Student Development, 44, 453-469.

Chen, H.T., (1990). Theory-driven evaluations, url=https://books.google.com/books?id=-hRsvHu85jkC, SAGE Publications

Collins, P. H. (1986). Learning from the outsider within: The sociological significance of Black feminist thought. Social problems, 33(6), s14-s32.

Cook, D. A., \& Dixson, A. D. (2013). Writing critical race theory and method: A composite counterstory on the experiences of Black teachers in New Orleans post-Katrina. International Journal of 
Qualitative Studies in Education, 26(10), 1238-1258.

Crenshaw, K. (1991). Mapping the margins: Intersectionality, identity politics, and violence against women of color. Stanford Law Review, 43(6), 1241-1300.

Crenshaw, K. W., Gotanda, N., Peller, G., \& Thomas, K. (Eds.). (1995).

Critical race theory: Key writings that formed the movement. New York: New Press.

Creswell, J. W. (2014). Research design: Qualitative, quantitative and mixed methods approaches. Thousand Oaks, CA: Sage Publications.

Cross Jr, W. E. (1971). Toward a psychology of Black liberation: The Negro-to-Black conversion experience. Black World, 20(9), 13-27.

Cross Jr, W. E. (1991). Shades of black: Diversity in African-American identity. Temple University Press.

Cross Jr, W. E., \& Fhagen-Smith, P. (2001). Patterns of African American identity development: A life span perspective. New perspectives on racial identity development: A theoretical and practical anthology, 243-270.

Crotty, M. (1998). The foundations of social research: Meaning and perspective in the research process. Thousand Oaks, CA: Sage.

Decuir, J., \& Dixson, A. (2004). "So when it comes out, they aren't that surprised that it is there": Using critical race theory as a tool of analysis of race and racism in education. Educational Researcher, $33,26-31$.

Delgado, R. S. J.(2001). Critical race theory: An introduction. New York: New York University.

Delgado Bernal, D. (2013). Critical race theory, Latino critical theory, and critical racedgendered epistemologies: recognizing students of color as holders and creators of knowledge. In Thayer-Bacon, B. J., Stone, L. Sprecher, K. M (Eds). Education feminism: classic and contemporary readings (389-408). Albany, NY: SUNNY Press. 
DeTurk, S. (2006). The power of dialogue: Consequences of intergroup dialogue and their implications for agency and alliance building. Communication Quarterly, 54(1), 33-51.

Douglas, Ty-Ron M.O., \& Kmt G. Shockley. (2017). Truths, Triumphs, and Testaments of Hope when Campus and Community Voices Rise (Guest Editorial). The Journal of Negro Education, 86(3), 199-203. doi:10.7709/jnegroeducation.86.3.0199

Douglas, Ty-Ron M.O., \& Maxwell Little. (2017). Voices from the Field: What Does a Chief Diversity Officer Do When Voices Rise?: An Interview with Dr. Kevin McDonald, Chief Diversity Officer of the University of Missouri System and Vice-Chancellor for Inclusion, Diversity and Equity at the University of Missouri. The Journal of Negro Education, 86(3), 381-391. doi:10.7709/jnegroeducation.86.3.0381

Erikson Erik, H. (1950). Childhood and society. New York.

Erikson Erik, H. (1968). Identity: youth and crisis. New York.

Evans, N., Forney, D., Guido, F., Patton, L., Renn, K. Student development in college. San Francisco, CA:Jossey-bass, 2010.

Feagin, J. R., \& Vera, H. H. \& Imani, N. (1996) The agony of education: Black students at White colleges and universities.

Ferdman, B. M., \& Gallegos, P. I. (2001). Racial identity development and Latinos in the United States. New perspectives on racial identity development: A theoretical and practical anthology, $32-66$.

Ford, D. (2015, November 10). Jonathan Butler: Meet the man whose hunger strike flipped the script at Mizzou. https://www.cnn.com/2015/11/09/us/jonathan-butler-hunger-strike-missouriprofile/index.html

Freire, P. (1970/1993/2000). Pedagogy of the oppressed, 30th Anniversary ed. New York: Continuum 
González, K. P. (2002). Campus culture and the experiences of Chicano students in a predominantly White university. Urban Education, 37(2), 193-218.

Guiffrida, D. A. (2006). Toward a cultural advancement of Tinto's theory. The Review of Higher Education, 29(4), 451-472.

Gurin-Sands, C., Gurin, P., Nagda, B. R. A., \& Osuna, S. (2012). Fostering a commitment to social action: How talking, thinking, and feeling make a difference in intergroup dialogue. Equity \& Excellence in Education, 45(1), 60-79.

Hansen, C. (2018). Do chief diversity officers help diversify a university's faculty? This study found no Evidence. The Chronical of Higher Education, 1-4. https://www.chronicle.com/article/Do-Chief-Diversity-Officers/244460

Harper, S. R. \& Quaye, S. J. (2007). Student Organizations as Venues for Black Identity Expression and Development among African American Male Student Leaders. Journal of College Student Development 48(2), 127-144. Johns Hopkins University Press. Retrieved August 29, 2018, from Project MUSE database.

Head, P. (2015, September 12). I just want to say how extremely hurt and disappointed I am. [Facebook post] https://www.facebook.com/payton.head

Helms, J. E. (1992). A race is a nice thing to have: A guide to being a white person or understanding the white persons in your life. Topeka, KS: Content Communications

Helms, J. E. (1995). An update of Helm's White and people of color racial identity models. In Versions were presented at the Psychology and Societal Transformation Conference, U Western Cape, South Africa, Jan 1994, and at a workshop entitled" Helm's Racial Identity Theory, "Annual Multicultural Winter Roundtable, Teachers Coll-Columbia U, New York, Feb 1994.. Sage Publications, Inc.

Hurtado, S. \& Clayton-Pedersen, A. R. \& Allen, W. R. \& Milem, J. F. (1998). Enhancing Campus 
Climates for Racial/Ethnic Diversity: Educational Policy and Practice. The Review of Higher Education21(3), 279-302. Johns Hopkins University Press. Retrieved August 29, 2018, from Project MUSE database.

Hurtado, S., Dey, E. L., \& Trevino, J. G. (1994). Exclusion or self-segregation?: Interaction across racial/ethnic groups on college campuses. S. Hurtado.

Johnson, G., \& Puplampu, K. (2008). Internet use during childhood and the ecological techno-subsystem. Canadian Journal of Learning and Technology / La revue canadienne de l'apprentissage et de la technologie, 34(1).

Jones, V. A., \& Reddick, R. J. (2017). The heterogeneity of resistance: How Black students utilize engagement and activism to challenge PWI inequalities. The Journal of Negro Education, 86(3), 204-219.

Kezar, A. (2018). How Colleges Change: Understanding, Leading, and Enacting Change. New York, NY: Routledge.

Kovacs, K. (2015, November 2). Update: MU student embarks on hunger strike, demands Wolf's removal from office. https://www.columbiamissourian.com/news/higher education/update-mu-studentembarks-on-hunger-strike-demands-wolfe-s/article 35ab864a-8186-11e5-902b$\underline{6 f 136 a 45260 \mathrm{~b} \cdot h t m l}$

Krings, A., Austic, E. A., Gutiérrez, L. M. \& Dirksen, K. E. (2015) The Comparative Impacts of Social Justice Educational Methods on Political Participation, Civic Engagement, and Multicultural Activism, Equity \& Excellence in Education, 48:3, 403-

417, DOI: $\underline{10.1080 / 10665684.2015 .1057087}$

Kuh, G. D., \& Love, P. G. (2000). A cultural perspective on student departure. Reworking the student departure puzzle, 196-212.

Kuh, G. D., Kinzie, J., Schuh, J. H., \& Whitt, E. J. (2011). Student success in college: Creating conditions that matter. John Wiley \& Sons. 
Ladson-Billings, G., \& IV Tate, W. F. (1995). Toward a critical race theory of education. In Ball, S. J., Sociology of Education: theories and methods (ed)., Teachers College Record, 97(1):47-68.

Leath, S. \& Chavous, T. (2017). "We really protested": The Influence of Sociopolitical Beliefs, Political Self-efficacy, and Campus Racial Climate on Civic Engagement among Black College Students attending Predominantly White Institutions. The Journal of Negro Education, 86(3), 220-237. doi:10.7709/jnegroeducation.86.3.0220

Lewis, A. E., Chesler, M., \& Forman, T. A. (2000). The impact of" colorblind" ideologies on students of color: Intergroup relations at a predominantly White university. Journal of Negro Education, 7491.

Marcia, J. E. (1966). Development and validation of ego-identity status. Journal of personality and social psychology, 3(5), 551.

Marcia, J. (1980). E. (1980). Identity in adolescence. Handbook of adolescent psychology, 159-187.

McCabe, J. (2009). Racial and Gender Microaggressions on a Predominantly-White Campus:

Experiences of Black, Latina/o and White Undergraduates. Race, Gender \& Class, 16(1/2), 133-151. Retrieved from http://www.jstor.org/stable/41658864

McElderry, J. A. \& Hernandez Rivera, S. (2017). "Your Agenda Item, Our Experience”: Two Administrators' Insights on Campus Unrest at Mizzou. The Journal of Negro Education, 86(3), 318-337. doi:10.7709/jnegroeducation.86.3.0318

Merriam, S. B. (1998). Qualitative research and case study applications in education. Revised and expanded from case study research in education. San Francisco, CA: Jossey-Bass.

Miles, MB. \& Huberman, AM. (1994). Qualitative Data Analysis (2nd edition). Thousand Oaks, CA: Sage Publications.

Moos, R. H. (1973). Conceptualizations of human environments. American psychologist, 28(8), 652. 
Moos, R. H. (1979). Evaluating educational environments. Jossey-Bass Inc Pub.

Muñoz, C. (1989). Youth, identity, power: The Chicano movement. Verso.

Museus, S. D. (2008). Focusing on institutional fabric: Using campus culture assessments to enhance cross-cultural engagement. Creating inclusive environments for cross-cultural learning and engagement in higher education. Washington, DC: National Association of Student Personnel Administrators.

Museus, S. D. (2008). The role of ethnic student organizations in fostering African American and Asian American students' cultural adjustment and membership at predominantly White institutions. Journal of College Student Development, 49(6), 568-586.

Museus, S. D. (2010). Delineating the ways that targeted support programs facilitate minority students' access to social networks and development of social capital in college. Enrollment Management Journal, 4(3), 10-41.

Museus, S. D., \& Harris, F. (2010). The elements of institutional culture and minority college student success. Managing diversity:(Re) visioning equity on college campuses, 25-44.

Museus, S. D., \& Quaye, S. J. (2009). Toward an intercultural perspective of racial and ethnic minority college student persistence. The Review of Higher Education, 33(1), 67-94.

Museus, S. D. (2011). Generating Ethnic Minority Student Success (GEMS): A qualitative analysis of high-performing institutions. Journal of Diversity in Higher Education, 4(3), 147.

Ndemanu, M. T. (2017). Antecedents of College Campus Protests Nationwide: Exploring Black Student Activists' Demands. The Journal of Negro Education, 86(3), 238-251.

Noblit, G. W., \& Jay, M. (2010). Against the majoritarian story of school reform: The Comer Schools Evaluation as a critical race counternarrative. In M. Freeman (Ed.), Critical social theory and evaluation practice. New Directions for Evaluation, 127, 71-82.

Parker, L. (2004). Commentary: Can critical theories of or on race be used in evaluation research in education? New Directions for Evaluation 2004 (101):85-93 Wiley 
Parker, L. (2003). Critical Race Theory and Its Implications for Methodology and Policy Analysis in Higher Education Desegregation. Counterpoints, 195, 145-180. Retrieved from http://www.jstor.org/stable/42978085

Parker, L., \& Lynn, M. (2002). What's Race Got to Do With It? Critical Race Theory's Conflicts With and Connections to Qualitative Research Methodology and Epistemology. Qualitative Inquiry, $8(1), 7-22$.

Pascarella, E.T., \& Terenzini, P. T. (2005). How college affects students: A summary. In how college affects students (Chapter 11; pp. 571-626). San Francisco: Jossey-Bass.

Pascarella, E. T., Terenzini, P. T., \& Feldman, K. A. (1991). How college affects students. San Francisco , CA: Jossey-Bass.

Patton, M. Q. (2002). Qualitative Research \& Evaluation Methods. Thousand Oaks, CA: SAGE.

Pearson, M. (2015, November 10). A timeline of University of Missouri protests. https://www.cnn.com/2015/11/09/us/missouri-protest-timeline/index.html

Peterson, M. W. (1978). Black Students on White Campuses: The Impacts of Increased Black Enrollments.

Pitner, R. O., Priester, M., Lackey, R. \& Duvall, D. (2018) A Dedicated Diversity Course or an Infusion Model? Exploring Which Strategy Is More Effective in Social Work Pedagogy, Journal of Social Work Education, 54:1, 49-60, DOI: 10.1080/10437797.2017.1314839

Rendón, L. I., Jalomo, R. E., \& Nora, A. (2000). Theoretical considerations in the study of minority student retention in higher education. Reworking the student departure puzzle, 1, 127-156.

Rochester, J. M. (2019). The Mizzou Meltdown: How a President Lost Control. Academic Questions, 32(1), 40-46.

Rowe, W., Bennett, S. K., \& Atkinson, D. R. (1994). White racial identity models: A critique and 
alternative proposal. The Counseling Psychologist, 22(1), 129-146.

Saldaña, J. (2016). The coding manual for qualitative researchers. Thousand Oaks, CA: Sage.

Solorzano, D., Ceja, M., \& Yosso, T. (2000). Critical race theory, racial microaggressions, and campus racial climate: The experiences of African American college students. Journal of Negro Education, 60-73.

Steele, C. M., Spencer, S. J., \& Aronson, J. (2002). Contending with group image: The psychology of stereotype and social identity threat. In Advances in experimental social psychology (Vol. 34, pp. 379-440). Academic Press.

Sue, D. W., \& Sue, D. (2012). Counseling the culturally diverse: Theory and practice. John Wiley \& Sons.

Sue, D. W., Capodilupo, C. M., Torino, G. C., Bucceri, J. M., Holder, A., Nadal, K. L., \& Esquilin, M. (2007). Racial microaggressions in everyday life: Implications for clinical practice. American psychologist, 62(4), 271.

Sunne, S. (2010, April 29). Students sentenced for cotton ball incident. https://www.themaneater.com/stories/campus/students-sentenced-cotton-ball-incident

Tierney, W. G. (1992). An anthropological analysis of student participation in college. The Journal of Higher Education, 63(6), 603-618.

Tierney, W. G. (1999). Models of minority college-going and retention: Cultural integrity versus cultural suicide. Journal of Negro Education, 80-91.

Torres, V., Howard-Hamilton, M., \& Cooper, D. L. (2003). Identity development of diverse populations: Implications for teaching and practice. Monograph for ASHE/ERIC. 
Treviño, J. G. (1993). Participation in ethnic/racial student organizations. (cited as 1992)

University of Missouri System. About the University of Missouri system. https://www.umsystem.edu/about-us

University of Missouri Mizzou. History of Mizzou. https://missouri.edu/about/history

University of Missouri Mizzou. https://missouri.app.box.com/v/enrollment-se-total

University of Missouri Mizzou. https://missouri.app.box.com/v/faculty-by-ethnicity

Watkins Liu, C. (2017). The Anti oppressive value of critical race theory and intersectionality in social movement study, Sociology of Race and Ethnicity, 4(3), pp. 1-16. https://doi.org/10.1177/2332649217743771

WCPO ABC 9, (2016, September 6). NKU students respond to racially charged flier for "White Week." https://www.wcpo.com/news/local-news/colleges/nku-news/nku-students-respond-to-raciallycharged-flier-for-white-week

Weick, K. E., \& Quinn, R. E. (1999). Organizational change and development. Annual Review of Psychology, 50, 361-386. https://doi.org/10.1146/annurev.psych.50.1.361

Yin, R. K. (2009). Case study research: Design and Methods. Thousand Oaks, CA: Sage Publications.

Zúñiga, X., Naagda, B. R. A., \& Sevig, T. D. (2002). Intergroup dialogues: An educational model for cultivating engagement across differences. Equity \&Excellence in Education, 35(1), 7-17.

Zúñiga, X., Lopez, G. E., \& Ford, K. A. (2012). Intergroup dialogue: Critical conversations about difference, social identities, and social justice: Guest editors' introduction. Equity \& Excellence in Education, 45(1), 1-13. 


\section{Appendix A}

\section{Dataset: Interview Protocol for Faculty, Instructor/Curriculum developer}

To the Interviewee: Hello

], thank you for agreeing to take this interview with me. My ultimate goal is to better understand the perceptions around the institutional response to the student led protest of 2015 at the University of Missouri-Columbia as a result of campus unrest stemming from a series of racist incidents. In doing so, I am particularly interested in studying the perceptions of the curricular interventions created as a response to the Black students' demand for the implementation of some form of a racial awareness and inclusion curriculum. Specifically, I am gathering data from both instructors who either taught/helped to develop the course or students who took the course. Someone like yourself, who either serve(d) as a faculty/instructor/developer of the curriculum, can provide some critical data as it pertains to the perceptions

Interview \#:

Interviewer: Bruce E. Mitchell II

Interviewee: faculty, instructor, curriculum developer

Date:

County: United States of America

\section{Location: Virtual}

Time: to

of this course from your perspective in order to inform future implications for this subject matter in similar higher education or institutional settings. So I have a series of guiding questions as it relates to: the creation of the course, purpose of the course, curriculum development, course requirement status, course assignments, implementation process, instructor qualifications or preparedness, personal perceptions of the course, perceptions of students' learning and behaviors etc. Feel free to share other details or information you think would be useful or not.

1. Bruce: Please talk about what led to the creation of courses situated around race at the University of Missouri-Columbia following the protests of 2015. Where these courses direct institutional responses to the demands of Black students and how widely known is this across the university community?

2. Bruce: (For a curriculum developer of the course(s) How did you begin crafting the content for each of the courses and what led to your decisions? Let's start with Citizenship @Mizzou followed by Race and the American Story.

3. Bruce: What do you believe the purpose was for both/either Citizenship @Mizzou and Race and the American Story?

4. Bruce: Given the list of demands from the Concerned Students of 1950 to have a required racial awareness and inclusion curriculum, how did this aid or impede upon your work with course development (if applicable) or your ability to create an atmosphere for learning and to be successful achieving student learning objectives?

- Citizenship @Mizzou

- Race and the American Story

5. Bruce: Talk about the course assignments, format, projects, and activities for learning and your perceptions of their usefulness and receptibility by the students.

6. Bruce: Please talk about the implementation process for getting the courses added to the curriculum as either required or optional and what support or barriers may have been encountered along the way.

7. Bruce: Given the sensitive nature of the subject matter, please talk about the qualifications and resources to gauge or ensure teacher preparedness for instructing or facilitating Citizenship @ Mizzou and/or Race and the American Story?

8. Bruce: looking back on the course(s), what are your perceptions about how those who taught or helped facilitate did in order to achieve student learning outcomes? What do you think was achieved? What do you think was done very well, poorly or needs improvement?

9. Bruce: What are your perceptions about what students who completed the course(s) feel or are able to do etc. as it pertains to race and perhaps the discontinuities of race dynamics on their campus and beyond?

10. Bruce: What was the racial demographic makeup of the Race and the American Story Course? 


\section{Appendix B}

Interview \#:

Date: June [ ], 2020

Location: Virtual
Interviewer: Bruce E. Mitchell II

Interviewee: Students, student facilitators or participants of the curricular intervention

County: United States of America

Time: EDT to

\section{Dataset: Interview Protocol for Students/Student Facilitators/Participants}

To the Interviewee: Hello [ ] thank you for agreeing to take this interview with me. My ultimate goal is to better understand the perceptions around the institutional response to the student led protest of 2015 at the University of Missouri-Columbia as a result of campus unrest stemming from a series of racist incidents. In doing so, I am particularly interested in studying the perceptions of the curricular interventions created as a response to the Black students' demand for the implementation of some form of a racial awareness and inclusion curriculum. Specifically, I am gathering data from both instructors who either taught/helped to develop the course or students who took the course. Someone like yourself, who either serve(d) as a faculty/instructor/developer of the curriculum, can provide some critical data as it pertains to the perceptions of this course from your perspective in order to inform future implications for this subject matter in similar higher education or institutional settings. So I have a series of guiding questions as it relates to: appreciation for the course, course necessity for students, curricular assignments, course requirement designation, instructional strategies/activities, instructor preparedness and aptness of subject matter, personal perceptions of the course, perceptions of students' learning and behaviors etc. Feel free to share other details or information you think would be useful or not. FIRST, do you grant permission for your identity to be used in the final published documents/dissertation?

1. Bruce: Can you talk about the impetus for the reforms? As a student facilitator, would you please describe the process of creating and implementing? (Who was involved, internal debates, discussions, etc.)

2. Bruce: How did you come to know about the course(s) Citizenship @Mizzou and/or Race and the American Story and what led you to choose it if not required or to help facilitate if applicable?

3. Bruce: Can you talk about the overall purpose of the curriculum for [ ] and some of the most valuable or important inclusions in the course? (In other words, share with us the most powerful or meaningful experiences)

4. Bruce: Please talk about your perceptions of the course(s) necessity for students to enroll and your thoughts around the courses' designation to be either required or optional? Does it take away from the desire to learn if it is required?

5. Bruce: What racial demographic(s) do you perceive as needing the course most if at all? Does the courses(s) reach the students who really need it most? Why or why not?

- $\quad$ Citizenship @Mizzou

- $\quad$ Race and the American Story

6. Bruce: How do you perceive the course(s)' instructional strategies, assignments, activities and projects as fulfilling its purpose to create learning opportunities around race and racism and how well do you think the course(s) influence behaviors to combat, advocate or face race and racism?

7. Bruce: What are your perceptions about instructor preparedness and their aptness to each or facilitate the course(s) around race? How would you describe the courses learning materials and instruction? (Surface level, intermediate or in depth?) What might you suggest differently for instructor/facilitator selection for the future if anything?

8. Bruce: How do you perceive either course (that you have taken or facilitated) to improve the institutions' campus racial climate? And what are your thoughts about the adequacy of these courses as an institutional response to the campus racial climate issues and following the protest of 2015 ?

9. Bruce: What advice would you give to other institutions trying to implement similar reforms. 


\section{Appendix C}

To: The University of Missouri October 20, 2015

During the University of Missouri's 104th homecoming parade, Saturday, October 10, 2015, eleven Black student leaders on campus interjected themselves into the parade, presenting UM system president, Tim Wolfe, and the Columbia community with a demonstration addressing Mizzou's history of racial violence and exclusivity. The demonstration covered the raw, painful, and often silenced history of racism and discrimination on the University of Missouri's campus. This history of racism at Mizzou dates back to 1935 when Lloyd Gaines petitioned the university to be its first Black law student and was denied admission. The actual year that the first Black student, Gus T. Ridgel, was accepted in the University of Missouri wasn't until 1950, hence where the concept of "Concerned Student 1950" comes from.

Concerned Student 1950, thus, represents every Black student admitted to the University of Missouri since then and their sentiments regarding race-related affairs affecting their lives at a predominantly white institution. Not only do our white peers sit in silence in the face of our oppression but also our administrators who perpetuate that oppression through their inaction. The Black experience on Mizzou's campus is cornered in offices and rarely attended to until it reaches media. Then, and only then, do campus administrators seek reactionary initiatives to attest to the realities of oppressed students, faculty, and staff. These temporary adjustments to the university's behaviors are not enough to assure that future generations of marginalized students will have a safe and inclusive learning experience during their time at Mizzou.

It is important to note that, as students, it is not our job to ensure that the policies and practices of the University of Missouri work to maintain a safe, secure and unbiased campus climate for all of its students. We do understand, however, that change does not happen without a catalyst. Concerned Student 1950 has invested time, money, intellectual capital, and excessive energy to bring to the forefront these issues and to get administration on board so that we, as students, may turn our primary focus back to what we are on campus to do: obtain our degrees.

The following document presents the demands of Concerned Student 1950. This document reflects the adjustments that we feel should be made to the University. We expect a response to these demands by 5:00pm on October 28, 2015.

If we do not receive a response to these demands by the date above, we will take appropriate nonviolent actions. If there are any questions, comments or concerns, you may forward them to ConcernedStudent1950@gmail.com.

The struggle continues,

\section{Concerned Student 1950}




\title{
Appendix C (Continued)
}

\author{
List of Demands
}

I. We demand that the University of Missouri System President, Tim Wolfe, writes a handwritten apology to the Concerned Student 1-9-5-0 demonstrators and holds a press conference in the Mizzou Student Center reading the letter. In the letter and at the press conference, Tim Wolfe must acknowledge his white male privilege, recognize that systems of oppression exist, and provide a verbal commitment to fulfilling Concerned Student 1-9-5-0 demands. We want Tim Wolfe to admit to his gross negligence, allowing his driver to hit one of the demonstrators, consenting to the physical violence of bystanders, and lastly refusing to intervene when Columbia Police Department used excessive force with demonstrators.

II. We demand the immediate removal of Tim Wolfe as UM system president. After his removal a new amendment to UM system policies must be established to have all future UM system president and Chancellor positions be selected by a collective of students, staff, and faculty of diverse backgrounds.

III. We demand that the University of Missouri meets the Legion of Black Collegians' demands that were presented in 1969 for the betterment of the black community.

IV. We demand that the University of Missouri creates and enforces comprehensive racial awareness and inclusion curriculum throughout all campus departments and units, mandatory for all students, faculty, staff, and administration. This curriculum must be vetted, maintained, and overseen by a board comprised of students, staff, and faculty of color.

V. We demand that by the academic year 2017-2018, the University of Missouri increases the percentage of black faculty and staff campus-wide to $10 \%$.

VI. We demand that the University of Missouri composes a strategic 10 year plan by May 1, 2016 that will increase retention rates for marginalized students, sustain diversity curriculum and training, and promote a more safe and inclusive campus.

VII. We demand that the University of Missouri increases funding and resources for the University of Missouri Counseling Center for the purpose of hiring additional mental health professionals; particularly those of color, boosting mental health outreach and programming across campus, increasing campus-wide awareness and visibility of the counseling center, and reducing lengthy wait times for prospective clients.

VIII. We demand that the University of Missouri increases funding, resources, and personnel for the social justices centers on campus for the purpose of hiring additional professionals, particularly those of color, boosting outreach and programming across campus, and increasing campus-wide awareness and visibility. 


\section{Appendix D}

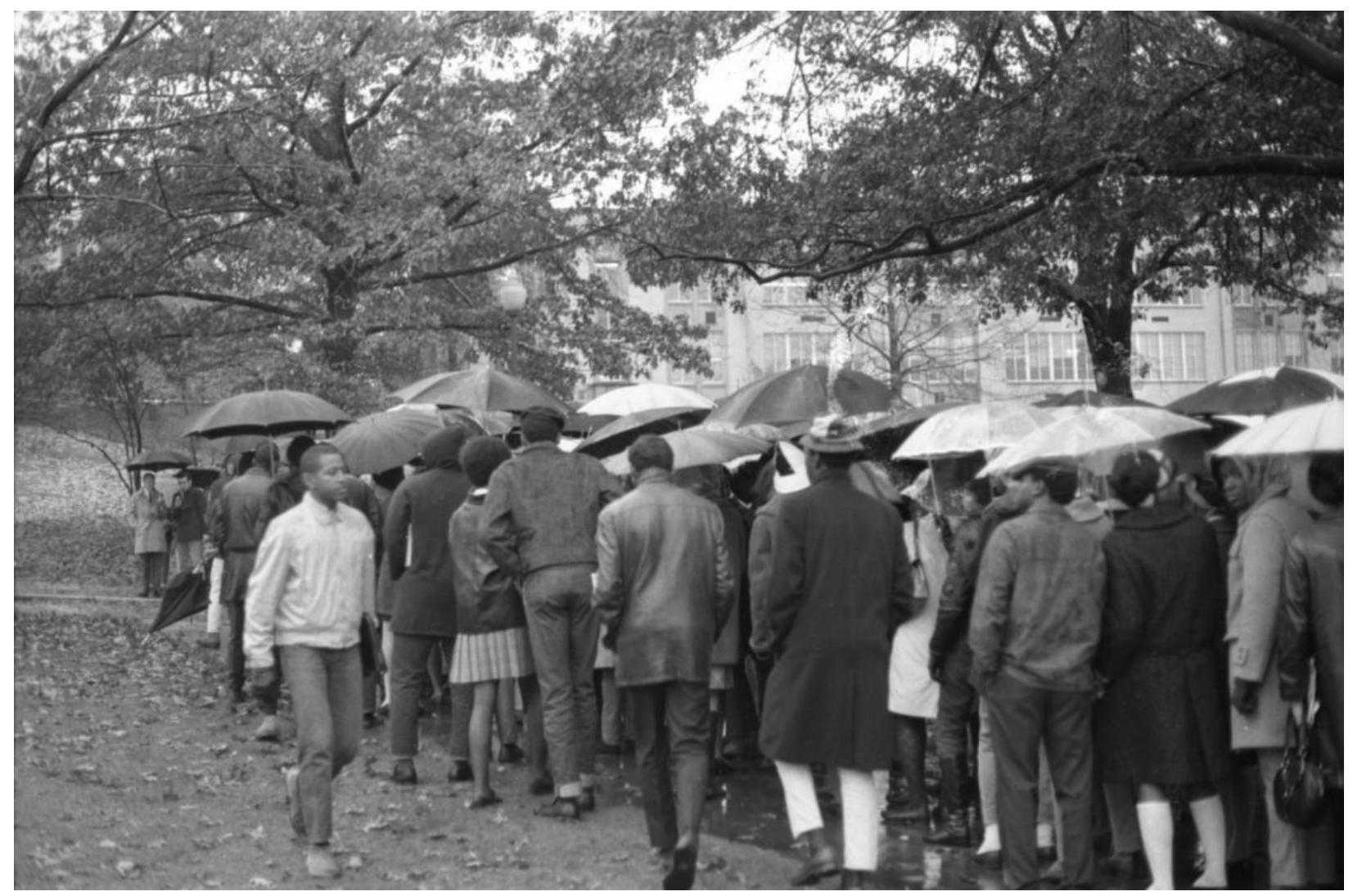

Black Student Walkout, 1968," Kent State University Libraries. Special Collections and Archives, accessed February 21, 2021, https://omeka.library.kent.edu/special-collections/items/show/2591. Photographer: Toliver, Lafayette 


\section{Appendix E}

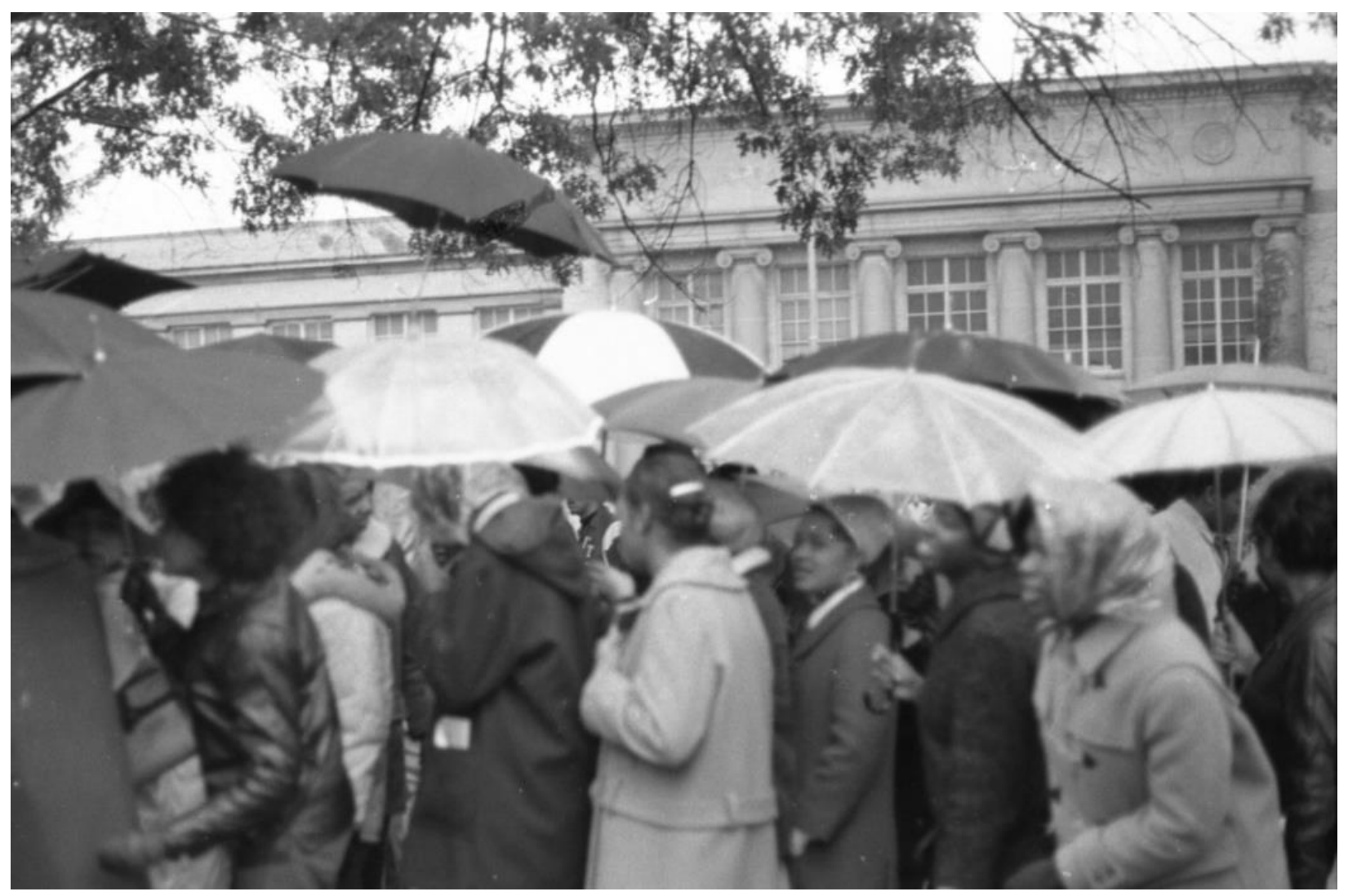

“Mass Black Student Walkout, 1968," Kent State University Libraries. Special Collections and Archives, accessed February 21, 2021. https://omeka.library.kent.edu/specialcollections/items/show/2592. Photographer: Toliver, Lafayette 


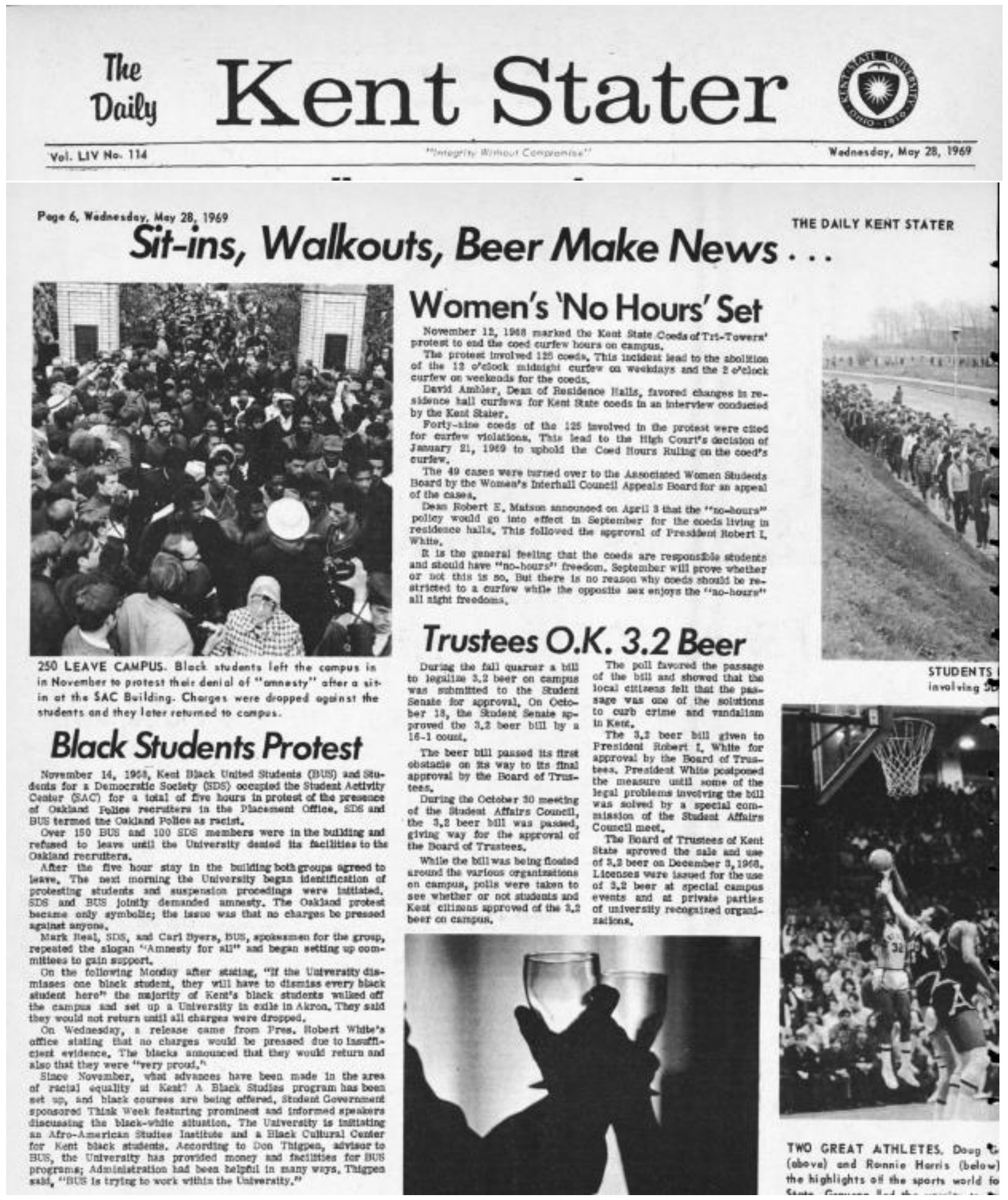

https://dks.library.kent.edu/?a=d\&d=dks19690528-01.2.22\&srpos=1\&e=------196-en-20--1--txt-txIN+Black+student+protest+oakland+police+1968------- 


\section{"Welcome White Week" Flyer At A Kentucky University Sparks Student Protest}

Northern Kentucky University is investigating the flyer which appeared to mock a

"Welcome Black Week" event on campus.

Tasneem Nashrulla

BuzzFeed News Reporter

Posted on September 7, 2016, at 5:04 p.m. ET

\section{y Tweet If Share 2 copy}

The flyer, shared on social media, appeared to mock the "Welcome Black Week" event organized by the African American Programs and Services - a student organization at NKU. The "White Week" poster listed events such as "White Lives Matter vs. Black Lives Matter," "Pizza Party for Tolerance" and "L.G.B.T.Q.R.S.T.U.V. and You."

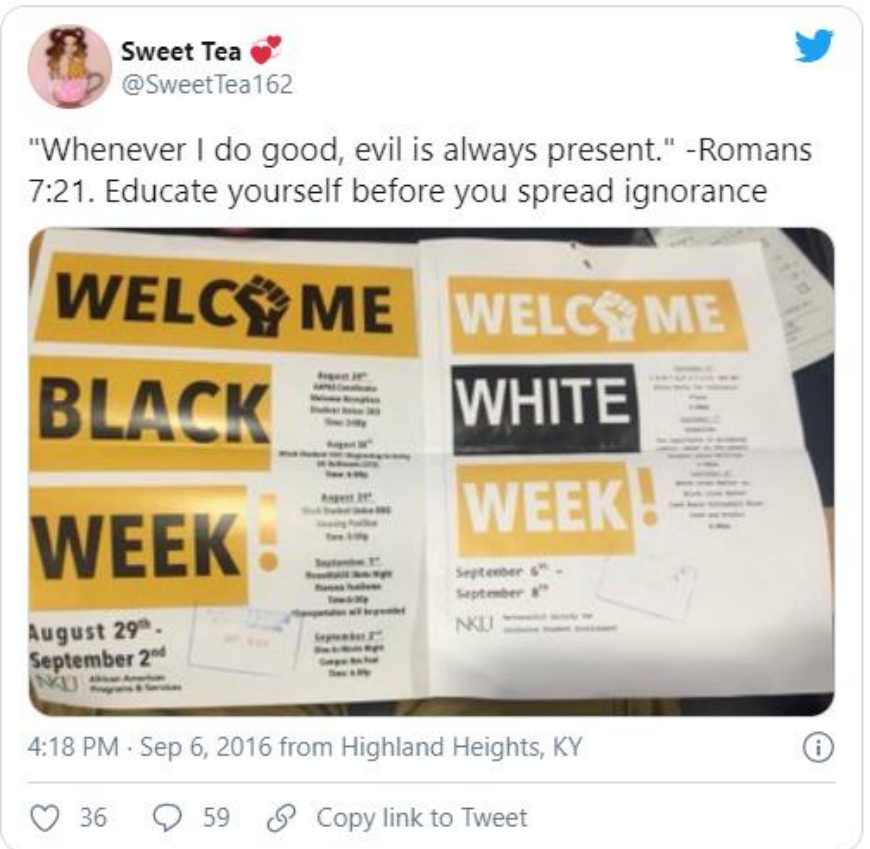

https://www.buzzfeednews.com/article/tasneemnashrulla/welcome-white-week-flyer-kentuckyuniversity 


\section{"Welcome White Week" Flyer At A Kentucky University Sparks Student Protest}

Northern Kentucky University is investigating the flyer which appeared to mock a "Welcome Black Week" event on campus.

Tasneem Nashrulla

BuzzFeed News Reporter

Posted on September 7, 2016, at 5:04 p.m. ET

\section{Tweet If Share o copy}

James Johnson, the protest's organizer, addressed other issues of racial discrimination on campus, including the "whitewashing" of events during Welcome Week and homecoming.

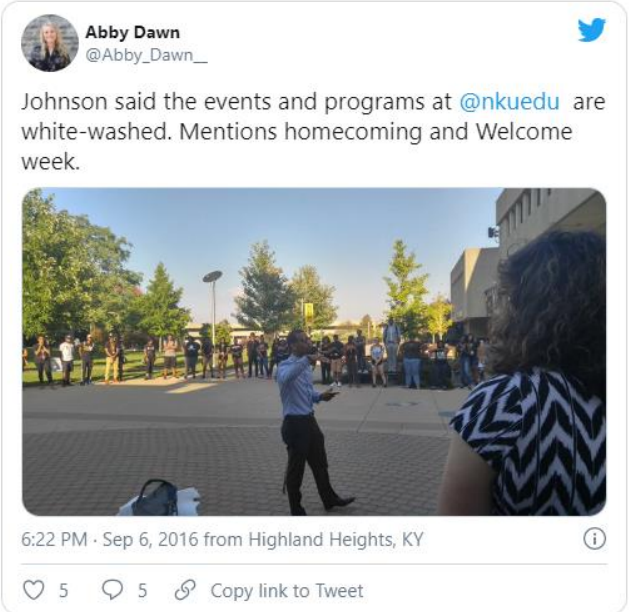

$$
\begin{aligned}
& \text { "The ignorance that stands on NKU's campus is no } \\
& \text { longer acceptable," Johnson told The Northerner, the } \\
& \text { student newspaper. "It's starting to become deliberate. } \\
& \text { Just like this person who deliberately copied our flyer," } \\
& \text { he said. } \\
& \text { Johnson said that the reason Welcome Black Week was } \\
& \text { organized was "because we weren't initially included in } \\
& \text { the festivities the university has." } \\
& \text { "We worked hard for Welcome Black Week in response } \\
& \text { to the lack of representation in the universal programs } \\
& \text { within Welcome Week, and we can even go back to } \\
& \text { homecoming," he told The Northerner. "It's like a cause } \\
& \text { and effect. So the reason they did this [posted the } \\
& \text { "Welcome White Week" flyer] was because we had } \\
& \text { Welcome Black Week." } \\
& \text { In a Facebook post referring to the protest, Johnson } \\
& \text { wrote, "Racism and discrimination must go! We will not } \\
& \text { stand for the undermining and the deliberate } \\
& \text { disrespect of our struggle. OUR VOICE WILL BE } \\
& \text { HEARD!" }
\end{aligned}
$$




\section{Appendix I}

\begin{tabular}{|c|c|}
\hline \multicolumn{2}{|c|}{$\begin{array}{c}\text { Exhibit 7.1 } \\
\text { Basic Human Resource Strategies. }\end{array}$} \\
\hline HUMAN RESOURCE PRINCIPLE & SPECIFIC PRACTICES \\
\hline Building and implement an HR strategy. & $\begin{array}{l}\text { - Develop a shared philosophy for } \\
\text { managing people. } \\
\text { - Build systems and practices to implement } \\
\text { the philosophy. }\end{array}$ \\
\hline Hire the right people. & $\begin{array}{l}\text { - Know what you want. } \\
\text { - Be selective. }\end{array}$ \\
\hline Keep them. & $\begin{array}{l}\text { - } \quad \text { Reward well. } \\
\text { - } \quad \text { Protect jobs. } \\
\text { - } \quad \text { Promote from within. } \\
\text { - } \quad \text { Share the wealth. }\end{array}$ \\
\hline Invest in them. & $\begin{array}{l}\text { - Invest in learning. } \\
\text { - Create development opportunities. }\end{array}$ \\
\hline Empower them. & $\begin{array}{l}\text { - } \quad \text { Provide information and support. } \\
\text { - } \quad \text { Encourage autonomy and participation. } \\
\text { - } \quad \text { Redesign Work. } \\
\text { - } \quad \text { Promote self-managing teams. } \\
\end{array}$ \\
\hline Promote diversity. & $\begin{array}{l}\text { Be explicit and consistent about the } \\
\text { organization's diversity philosophy. } \\
\text { - Hold managers accountable. }\end{array}$ \\
\hline
\end{tabular}


Appendix J

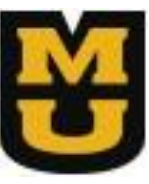

\title{
Evaluation of Instruction and Course
}

\author{
University of Missouri
}

당

Assessment Resource Center

Complete this COVER SHEET and place on top of each group of evaluations.

Department: Black Stwdes

$\begin{array}{lll}\text { Contact Person: Nar Beth Brown } & \text { Phone: } 573-882-1229 \\ \text { Instructor Name: Dr. Stephen Grawes } & \end{array}$

Catalog Number and Section: (Example: 1000.04) BL-StW 2425.02

DIRECTIONS:

1. Use a \#2 black lead pencil.

2. Write the correct number in the box and fill in the corresponding bubble below.

3. Darken the bubbles completely.

5. Erase completely or use correction tape (NOT a liquid) to make any corrections.

REQUIRED INFORMATION:

This information must be correct. Electronic reports (PDFs) cannot be generated from the accompanying - 8 digits

This is the employee ID number of the instructor of this class. If ID number has leading Os, these zeros must be entered so that all columns have à number. For student instructors, use their student ID number.
Reminder. To avoid errors. Confirm that the instructor is listed correctly in Peoplesoft before submitting evaluation forms.

-5 digits This number is in the Current Class Offerings (Schedule of Classes), column heading "Class.". Each
class session (e.g.. lecture, lab, discussion) will have a unique number. This number will be different each semester.

The semester the class was taught. The year will automatically be added.

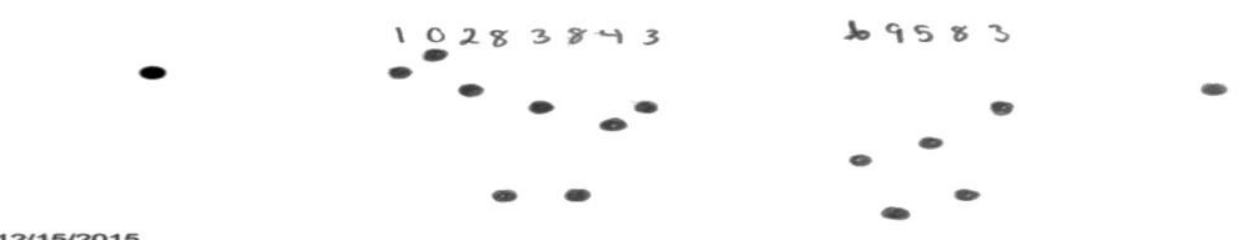




\section{Appendix J (Continued)}

\section{Group Report for: Graves,Stephen Charles Wesley; Course: RACE \& THE AMERICAN STORY}

Course: BL_STU 2425 Section: 02 Semester: SP2018

Class Number: 69583 No. Respondents: 15

\begin{tabular}{|c|c|c|c|c|c|c|c|}
\hline Standard Form & & & & & & & \\
\hline Choices: Strongly Agree, Agree, Neutral, Disagree, Strongly Disagree & Percen & it of $\operatorname{Re}$ & ponses & & & & \\
\hline Course Content and Structure & SA (5) & A (4) & $N(3)$ & $\mathrm{D}(2)$ & SD (1) & \# Rsp & Mean \\
\hline $\begin{array}{l}\text { The syllabus clearly explained the course objectives, requirements, and } \\
\text { grading system. }\end{array}$ & $47 \%$ & $47 \%$ & $0 \%$ & $7 \%$ & $0 \%$ & 15 & 4.33 \\
\hline $\begin{array}{l}\text { Course content was relevant and useful (e.g., readings, online media, } \\
\text { classwork, assignments). }\end{array}$ & $67 \%$ & $33 \%$ & $0 \%$ & $0 \%$ & $0 \%$ & 15 & 4.67 \\
\hline $\begin{array}{l}\text { Resources (e.g., articles, literature, textbooks, class notes, online } \\
\text { resources) were easy to access. }\end{array}$ & $93 \%$ & $7 \%$ & $0 \%$ & $0 \%$ & $0 \%$ & 15 & 4.93 \\
\hline This course challenged me. & $67 \%$ & $33 \%$ & $0 \%$ & $0 \%$ & $0 \%$ & 15 & 4.67 \\
\hline Teaching Delivery & SA (5) & $A(4)$ & $\mathrm{N}(3)$ & $\mathrm{D}(2)$ & SD (1) & \# Rsp & Mean \\
\hline This instructor was consistently well-prepared. & $87 \%$ & $13 \%$ & $0 \%$ & $0 \%$ & $0 \%$ & 15 & 4.87 \\
\hline This instructor was audible and clear. & $80 \%$ & $13 \%$ & $7 \%$ & $0 \%$ & $0 \%$ & 15 & 4.73 \\
\hline This instructor was knowledgeable and enthusiastic about the topic. & $93 \%$ & $7 \%$ & $0 \%$ & $0 \%$ & $0 \%$ & 15 & 4.93 \\
\hline $\begin{array}{l}\text { This instructor effectively used examples/illustrations to promote } \\
\text { learning. }\end{array}$ & $73 \%$ & $20 \%$ & $7 \%$ & $0 \%$ & $0 \%$ & 15 & 4.67 \\
\hline This instructor fostered questions and/or class participation. & $93 \%$ & $7 \%$ & $0 \%$ & $0 \%$ & $0 \%$ & 14 & 4.93 \\
\hline This instructor clearly explained important information/ideas/concepts. & $67 \%$ & $33 \%$ & $0 \%$ & $0 \%$ & $0 \%$ & 15 & 4.67 \\
\hline $\begin{array}{l}\text { This instructor effectively used teaching methods appropriate to this } \\
\text { class (e.g., critiques, discussion, demonstrations, group work). }\end{array}$ & $67 \%$ & $33 \%$ & $0 \%$ & $0 \%$ & $0 \%$ & 15 & 4.67 \\
\hline Learning Environment & SA (5) & $A(4)$ & $N(3)$ & $\mathrm{D}(2)$ & $\mathrm{SD}(1)$ & \# Rsp & Mean \\
\hline This instructor responded appropriately to questions and comments. & $60 \%$ & $40 \%$ & $0 \%$ & $0 \%$ & $0 \%$ & 15 & 4.60 \\
\hline This instructor stimulated student thinking and learning. & $80 \%$ & $20 \%$ & $0 \%$ & $0 \%$ & $0 \%$ & 15 & 4.80 \\
\hline $\begin{array}{l}\text { This instructor promoted an atmosphere of mutual respect regarding } \\
\text { diversity in student demographics and viewpoints, such as race, } \\
\text { gender, or politics. }\end{array}$ & $87 \%$ & $7 \%$ & $7 \%$ & $0 \%$ & $0 \%$ & 15 & 4.80 \\
\hline This instructor was approachable and available for extra help. & $53 \%$ & $40 \%$ & $7 \%$ & $0 \%$ & $0 \%$ & 15 & 4.47 \\
\hline This instructor used class time effectively. & $73 \%$ & $27 \%$ & $0 \%$ & $0 \%$ & $0 \%$ & 15 & 4.73 \\
\hline $\begin{array}{l}\text { This instructor helped students to be independent learners, responsible } \\
\text { for their own learning. }\end{array}$ & $60 \%$ & $40 \%$ & $0 \%$ & $0 \%$ & $0 \%$ & 15 & 4.60 \\
\hline Assessment & SA (5) & A (4) & $N(3)$ & $\mathrm{D}(2)$ & $\mathrm{SD}(1)$ & \# Rsp & Mean \\
\hline I was well-informed about my performance during this course. & $53 \%$ & $20 \%$ & $7 \%$ & $20 \%$ & $0 \%$ & 15 & 4.07 \\
\hline & ln $70 /$ & $220 \%$ & $12 \%$ & no/ & $70 /$ & $\left.\right|_{15}$ & \\
\hline
\end{tabular}




\begin{tabular}{|l|lll|l|}
\hline Feedback for Other Students (IDK = I Don't Know) & $\%$ Yes \% No \% IDK & \# Rsp \\
\hline Would you recommend this class to other students regarding...? & & & \\
\hline CLASS CONTENT & $93 \%$ & $7 \%$ & $0 \%$ & 15 \\
\hline CLASS STRUCTURE (E.G., ORGANIZATION, PACING) & $87 \%$ & $13 \%$ & $0 \%$ & 15 \\
\hline POSITIVE LEARNING ENVIRONMENT & $93 \%$ & $7 \%$ & $0 \%$ & 15 \\
\hline INSTRUCTOR'S TEACHING SKILL/STYLE & $87 \%$ & $13 \%$ & $0 \%$ & 15 \\
\hline FAIRNESS OF GRADING & $87 \%$ & $7 \%$ & $7 \%$ & 15 \\
\hline
\end{tabular}

\begin{tabular}{|c|c|c|c|c|c|c|c|c|c|c|c|c|c|}
\hline \multicolumn{14}{|c|}{ Student Information (NA = Not Applicable, NR = No Response) } \\
\hline \multicolumn{2}{|c|}{ Course } & \multicolumn{2}{|c|}{$\begin{array}{l}\text { Expected } \\
\text { Grade }\end{array}$} & \multicolumn{2}{|c|}{ Class Year } & \multicolumn{2}{|c|}{ Classes attend } & \multicolumn{2}{|c|}{$\begin{array}{l}\text { Extent use } \\
\text { online }\end{array}$} & \multicolumn{2}{|c|}{$\begin{array}{l}\text { Outside hours } \\
\text { per week }\end{array}$} & \multicolumn{2}{|c|}{ Complete work } \\
\hline $\begin{array}{l}\text { Requirem } \\
\text { en }\end{array}$ & $13 \%$ & $A$ & $80 \%$ & Freshman & $27 \%$ & $0-25$ & $0 \%$ & None & $93 \%$ & $0-3$ & $0 \%$ & $0-25$ & $7 \%$ \\
\hline Elective & $80 \%$ & $B$ & $7 \%$ & $\begin{array}{l}\text { Sophomo } \\
\text { e }\end{array}$ & $20 \%$ & $26-50$ & $0 \%$ & Little & $7 \%$ & $4-7$ & $0 \%$ & $26-50$ & $0 \%$ \\
\hline Other & $0 \%$ & C & $0 \%$ & Junior & $27 \%$ & $51-75$ & $0 \%$ & Some & $0 \%$ & $8-11$ & $20 \%$ & $51-75$ & $7 \%$ \\
\hline \multirow[t]{6}{*}{ NR } & $7 \%$ & D & $0 \%$ & Senior & $27 \%$ & $76-90$ & $7 \%$ & Moderate & $0 \%$ & $12-15$ & $47 \%$ & $76-90$ & $47 \%$ \\
\hline & & $F$ & $0 \%$ & Graduate & $0 \%$ & $91-100$ & $93 \%$ & Large & $0 \%$ & $>15$ & $20 \%$ & $91-100$ & $40 \%$ \\
\hline & & $S$ & $0 \%$ & Other & $0 \%$ & NA & $0 \%$ & NA & $0 \%$ & NA & $13 \%$ & NA & $0 \%$ \\
\hline & & $U$ & $0 \%$ & NR & $0 \%$ & NR & $0 \%$ & NR & $0 \%$ & NR & $0 \%$ & NR & $0 \%$ \\
\hline & & None & $0 \%$ & & & & & & & & & & \\
\hline & & NR & $13 \%$ & & & & & & & & & & \\
\hline
\end{tabular}

Grade $A \& B=$ The mean score of students who reported an expected grade of $A$ or $B$.

\begin{tabular}{|l|l|l|l|l|l|l|l|}
\hline \multicolumn{6}{|l|}{ Construct Means (20 Questions) } \\
\hline Content/Struct & \multicolumn{3}{l|}{ Teaching } & \multicolumn{3}{l|}{ Environment } & \multicolumn{2}{l|}{ Assessment } \\
\hline Mean & 4.65 & Mean & 4.78 & Mean & 4.67 & Mean & 4.18 \\
\hline $\begin{array}{l}\text { Grade A } \\
\text { \& B }\end{array}$ & 4.60 & $\begin{array}{l}\text { Grade A } \\
\text { \& B }\end{array}$ & 4.81 & $\begin{array}{l}\text { Grade A } \\
\text { \& B }\end{array}$ & 4.71 & $\begin{array}{l}\text { Grade A } \\
\text { \& B }\end{array}$ & 4.08 \\
\hline
\end{tabular}

\begin{tabular}{|l|l|}
\hline \multicolumn{2}{|c|}{ COMPOSITE SCOR } \\
\hline Mean & 4.63 \\
\hline $\begin{array}{l}\text { Grade A } \\
\text { \& B }\end{array}$ & 4.62 \\
\hline
\end{tabular}

Construct Means and Composite Score are calculated based on the number of respondents for each question in order to apply less weight to questions not applicable to a class. 